\title{
Improved Abstractions and Turnaround Time for FPGA Design Validation and Debug
}

\author{
Yousef Shafik Iskander \\ Dissertation submitted to the Faculty of the \\ Virginia Polytechnic Institute and State University \\ in partial fulfillment of the requirements for the degree of \\ Doctor of Philosophy \\ in \\ Computer Engineering \\ Cameron D. Patterson, Chair \\ Thomas L. Martin \\ Paul D. Plassmann \\ Sedki M. Riad \\ Ricardo A. Burdisso \\ August 6, 2012 \\ Blacksburg, Virginia
}

Keywords: FPGA, reconfigurable computing, debug, design validation, partial reconfiguration, development

Copyright 2012, Yousef S. Iskander 


\title{
Improved Abstractions and Turnaround Time for FPGA Design Validation and Debug
}

\author{
Yousef Shafik Iskander
}

(ABSTRACT)

Design validation is the most time-consuming task in the FPGA design cycle. Although manufacturers and third-party vendors offer a range of tools that provide different perspectives of a design, many require that the design be fully re-implemented for even simple parameter modifications or do not allow the design to be run at full speed. Designs are typically first modeled using a high-level language then later rewritten in a hardware description language, first for simulation and then later modified for synthesis. IP and third-party cores may differ during these final two stages complicating development and validation. The developed approach provides two means of directly validating synthesized hardware designs. The first allows the original high-level model written in $\mathrm{C}$ or $\mathrm{C}++$ to be directly coupled to the synthesized hardware, abstracting away the traditional gate-level view of designs. A high-level programmatic interface allows the synthesized design to be validated with the same arbitrary test data on the same framework as the hardware. The second approach provides an alternative view to FPGAs within the scope of a traditional software debugger. This debug framework leverages partially reconfigurable regions to accelerate the modification of dynamic, software-like breakpoints for low-level analysis and provides a automatable, scriptable, command-line interface directly to a running design on an FPGA.

This work is supported by DARPA and the United States Army under Contract Number W31P4Q08-C-0314. The views, opinions, and/or findings contained in this article/presentation are those of the author/presenter and should not be interpreted as representing the official views or policies, either expressed or implied, of the Defense Advanced Research Projects Agency or the Department of Defense. Approved for Public Release, Distribution Unlimited. 


\section{Dedication}

To my unconditionally loving and supportive parents, Shafik and Horia Iskander, to whom I owe so much in teaching me the value of education and making sacrifices for me to have one.

To my wife Tere and son Alex, who have brought me untold joy with their wonderful distractions. I wrote much of my preliminary exam while holding Alex as an infant so he could sleep, and took many breaks to play blocks while writing this dissertation. 


\section{Acknowledgments}

I am permanently indebted to many people for the completion of this dissertation. I have surely forgotten to mention someone, and am unable to properly express my gratitude to those I have listed.

My adviser, Cameron Patterson provided me with an opportunity when I had all but given up and provided exceptional support and meaningful insight which has been the real education. I am grateful for his mentoring. Thanks are also due to Dr. Jaime de la Ree for helping get past some particularly difficult obstacles. Thanks are of course due to all who served on my committee, Tom Martin, Sedki Riad, Paul Plassmann, and Ricardo Burdisso who held me to very high standards to which I can be proud of.

I'm indebted to my longtime friend Stephen Craven who not only encouraged me to continue when I was about to give up, but provided the motivation to return to school to pursue my doctorate, provided an opportunity to do so, and pushed me to do even better. He also provided invaluable support and advice throughout.

I'm grateful to the many special friends I made during my once in a lifetime stay in Los Alamos, New Mexico at the Los Alamos National Laboratory. Thanks are due to Kei

Davis, Heather Quinn, Zack Baker, Paul Graham, Sara del Valle, Hugh Greenberg, and Tony Salazar for selflessly sharing their knowledge, friendship, and experience; to M'hamed "Jebb" Jebbanema for his friendship and hiking trips through White Rock Canyon; and to 
Jim and Gabriela Stiner for memorable evenings at the "Stiner Diner" at their lovely home in White Rock.

I'm also grateful to Dr. Linda Wills of Georgia Tech, a patient and insightful woman to whom I owe so much for forming my first graduate school experience. She was instrumental in my desire to return to complete my doctorate and my success as graduate student. Her husband Scott was also an influential professor. Sadly, he passed away while I was writing this dissertation.

Thanks to the members of the Configurable Computing Machinery (CCM) Lab at Virginia Tech, including Neil Steiner, Lee Lerner, Tingting Meng, and the students who worked on this project with me: Athira Chandrasekharan, Suresh Raja Gopalan, Tony Frangieh, and Guruprasad Subbarayan. Thanks also to Brian Knight for his invaluable support on this project and his friendship.

Thanks is due to the always friendly staff of Sub Station II in Blacksburg, Va. I was sitting in that restaurant with my good friend Stephen Craven when I decided to attend Virginia Tech and it is also there I spent many enjoyable lunches thinking about my work. I highly recommend the $\# 9$.

I, like so many other in this field, are indebted to the authors of open-source software which is frequently used without credit or thanks to the many anonymous authors. Please see the Appendix for a listing of the open-source software packages that were instrumental in this research.

This work was performed at Virginia Tech in Blacksburg, Va. with support provided through Luna Innovations Incorporated in Roanoke, Va. 


\section{Contents}

List of Figures $\quad$ x

List of Tables $\quad$ xii

List of Listings $\quad$ xiii

Acronyms $\quad$ XV

1 Introduction $\quad 1$

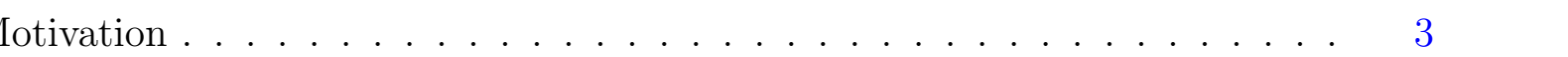

1.2 Problem Statement . . . . . . . . . . . . . . . . . . 4

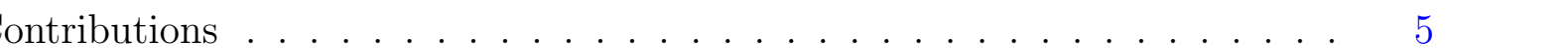

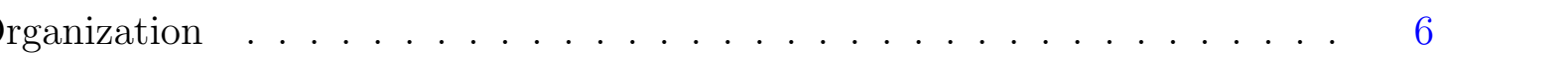

2 Background $\quad 9$

2.1 An Overview of Programmable Logic . . . . . . . . . . . . . . . . . 9

2.2 FPGA Development . . . . . . . . . . . . . . . . . 17 
2.2.1 Design Flow Overview . . . . . . . . . . . . . . . . 21

2.3 Debug Methodologies . . . . . . . . . . . . . . . . 29

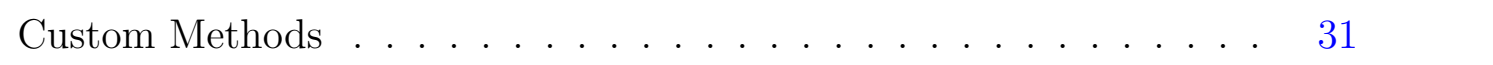

2.3.2 Simulator-Based Development . . . . . . . . . . . . . 32

2.3.3 Commercial Debug Solutions . . . . . . . . . . . . 33

2.3.4 Assertion-Based Verification ................ 35

2.4 High-Level Language Synthesis . . . . . . . . . . . . . . . . . . 36

2.5 Dynamic Runtime Reconfiguration . . . . . . . . . . . . . . . . 39

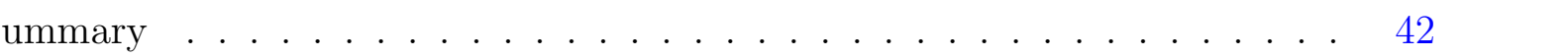

3 Related Work $\quad 44$

3.1 Categorization of Debug Approaches . . . . . . . . . . . . . . 44

3.2 Commercial Debug Products . . . . . . . . . . . . . . . 48

3.2.1 Vendor Products . . . . . . . . . . . . . . 48

3.2.2 Third-Party Products . . . . . . . . . . . . . . 48

3.3 Debug-Related Research . . . . . . . . . . . . . . . . . 50

3.4 High-Level Synthesis . . . . . . . . . . . . . . . . . . . . 53

3.5 Deficiencies in Existing Approaches . . . . . . . . . . . . . . . . 55

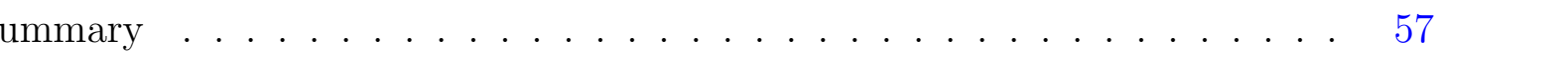


4.1 High-Level Validation . . . . . . . . . . . . . . . . . . . . . . . . . . . 62

4.1.1 Mapping Software Procedures to Hardware Modules . . . . . . . . . . 64

4.1.2 Mapping Data and Control Signals to the Software Model . . . . . . 65

4.2 Low-Level Debug . . . . . . . . . . . . . . . . . . . . . . . 66

4.2 .1 Improving Visibility $\ldots \ldots \ldots \ldots$. . . . . . . . . 67

4.2 .2 Improving Controllability _ . . . . . . . . . . . . . . . . . . 69

4.2 .3 Improving Agility . . . . . . . . . . . . . . . . . . . 72

4.3 Dynamic Modular Design and Validation . . . . . . . . . . . . . . . 73

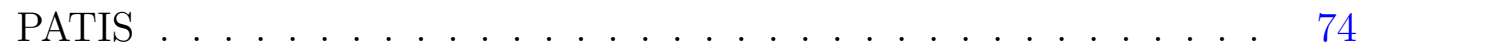

4.4 Summary . . . . . . . . . . . . . . . . . . . . . . 78

$\begin{array}{llr}5 & \text { Implementation } & 79\end{array}$

5.1 High-Level Validation . . . . . . . . . . . . . . . . . . . . . . . . 79

5.1.1 Reference Model Execution and Hardware Data Staging . . . . . . 81

5.1 .2 Test Harness and API $\ldots \ldots \ldots \ldots$

$5.2 \quad$ Low-Level Debug . . . . . . . . . . . . . . . . . . . . . . . . 86

5.2 .1 Programmable Debug Controller _. . . . . . . . . . . . . 88

$5.2 .2 \quad$ Unified Software Interface . . . . . . . . . . . . . . . . . 98

5.2 .3 Logic Model and Symbol Table Creation . . . . . . . . . . . . . . 102

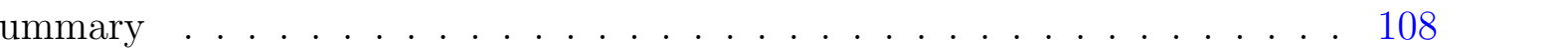


6.1 High-Level Validation . . . . . . . . . . . . . . . . . 111

6.1 .1 Secure Hash Algorithm . . . . . . . . . . . . . . . . . . . . 112

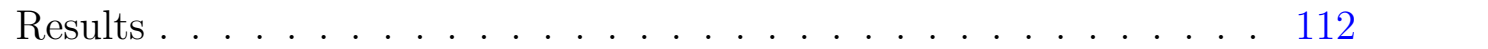

6.1.3 Summary and Future Work . . . . . . . . . . . . . . 115

6.2 Low-Level Debug . . . . . . . . . . . . . . . . . . . . . . . 116

6.2.1 Benchmark Designs . . . . . . . . . . . . . . 116

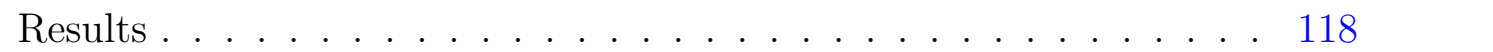

6.2.3 Summary and Future Work . . . . . . . . . . . . . . . . . 128

7 Conclusions and Future Work 131

7.1 Review of Contributions . . . . . . . . . . . . . . . 131

7.2 Future Work . . . . . . . . . . . . . . . . . . 134

$\begin{array}{ll}\text { Bibliography } & 135\end{array}$

A Open-Source and Free Software Acknowledgement 144

B Source Code $\quad 145$

B.1 High-Level Validation . . . . . . . . . . . . . . . . . . . . 145

B.2 Low-Level Debug . . . . . . . . . . . . . . . . . . . . 154 


\section{List of Figures}

1.1 Hardware development flow. . . . . . . . . . . . . . . . . . 5 5

2.1 Early programmable logic devices with discrete gates connected by programmable interconnects. . . . . . . . . . . . . . . . . . . . 10

2.2 Modern FPGAs organized as a regular array of resources. . . . . . . . . . . . 11

2.3 LUTs replace arbitrary logic with simple memories. . . . . . . . . . . . . 13

2.4 Overview of software development flow. . . . . . . . . . . . . . . . . . 19

2.5 Simplified view of FPGA development flow. . . . . . . . . . . . . . . 20

2.6 Detailed overview of FPGA development flow. . . . . . . . . . . . . . . 22

2.7 The model verification gap in hardware development. . . . . . . . . . . . . 30

2.8 Partial reconfiguration for multiple logic modules in the region. . . . . . . . 41

3.1 JTAG chains visit every resource in the FPGA. . . . . . . . . . . . . . 45

3.2 JTAG daisy chains require few connections. . . . . . . . . . . . . . 47

3.3 ChipScope gathers data through physically routed nets. . . . . . . . . . . 49

4.1 Refactoring code improves maintainability. . . . . . . . . . . . . . . 64 
4.2 Mapping data paths in hardware and software. . . . . . . . . . . . .

4.3 Developing a software model for hardware control signals from simulation models. . . . . . . . . . . . . . . . . . . . 66

4.4 a) A serial model of accessing register state; b) a random access model in which registers are accessible as memory locations. . . . . . . . . . . . 68

4.5 Symbol table mapping logical design elements to physical locations in an FPGA. 69

4.6 Schematic representation of a simple clock buffer. . . . . . . . . . . . 71

4.7 Accelerating debugging turnaround with a reconfigurable region for debugging

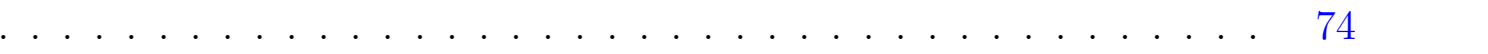

4.8 PATIS provides each top-level module its own reconfigurable region. . . . . . 76

4.9 Dynamic Modular Design flow. . . . . . . . . . . . . . . . . . 77

5.1 Overview of High-Level Validation. . . . . . . . . . . . . . . . . . 80

5.2 MicroBlaze architecture. . . . . . . . . . . . . . . . . . . 83

5.3 HLV capture window parameters. . . . . . . . . . . . . . . . 85

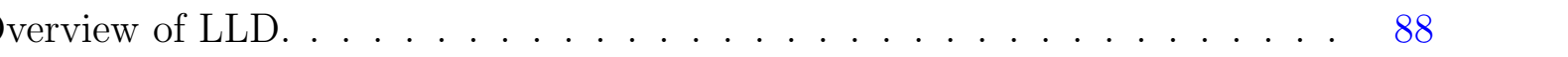

$5.5 \quad$ LLD clock control. . . . . . . . . . . . . . . . . . . . . . . 90

$5.6 \quad$ LLD breakpoint region detail. . . . . . . . . . . . . . . . . . . . 92

5.7 Relationship between device resources and configuration frames. . . . . . . . 99

5.8 Logic allocation record format. . . . . . . . . . . . . . . . . . . 102

5.9 Algorithm to retrieve register values. . . . . . . . . . . . . . 107

6.1 SHA-1 block diagram. . . . . . . . . . . . . . . . . . 113

6.2 Benchmark design implementation times. . . . . . . . . . . . . . . . . . 121

6.3 Percentage of time required for LLD implementation. . . . . . . . . . . . 123

6.4 Execution call graph of console application. . . . . . . . . . . . . . 130 


\section{List of Tables}

2.1 Timeline of logic density of Xilinx FPGAs. . . . . . . . . . . . . . 14

2.2 PAR times for non-trivial designs with typical timing goals. $\ldots \ldots \ldots 35$

3.1 Timeline of Xilinx Boundary Scan chain length. . . . . . . . . . . . . . 46

3.2 Summary of debug products. . . . . . . . . . . . . . . . 56

4.1 Truth table for a simple clock buffer. . . . . . . . . . . . . . . 71

5.1 Summary of HLV API. . . . . . . . . . . . . . . . . . 84

5.2 Register map for the HLV peripheral. . . . . . . . . . . . . . . 86

5.3 Register map for Programmable Debug Controller. . . . . . . . . . . . . . 89

5.4 Summary of on-chip debugger commands. . . . . . . . . . . . . 100

5.5 Summary of workstation commands. . . . . . . . . . . . . . . 110

6.1 Benchmark design implementation times (hours:minutes:seconds) . . . . . 120

6.2 LLD implementation times (minutes:seconds) . . . . . . . . . . . . . . . 122 


\section{Listings}

2.1 Verilog shift register definition from XST synthesizer documentation. . . . . 24

2.2 Implementation of a counter with HLS. . . . . . . . . . . . . . . . . 39

2.3 Synthesizable RTL generated from Listing 2.2. . . . . . . . . . . . . . 40

5.1 Sample HLV program. . . . . . . . . . . . . . . . . . . . . . 87

5.2 Breakpoint data structure. . . . . . . . . . . . . . . . 94

5.3 Generated Verilog breakpoint module for reconfigurable breakpoint region. . $\quad 95$

5.4 BitInfo data structure. . . . . . . . . . . . . . . . . . . . 104

6.1 Transcript of an HLV test session. . . . . . . . . . . . . . . . . . . . . 114

6.2 LLD debug transcript for a SHA-1 core. . . . . . . . . . . . . . . . . . 124

B.1 HLV User Logic. . . . . . . . . . . . . . . . . . . . . . . 145

B.2 SHA Test Code. . . . . . . . . . . . . . . . . . . 149

B.3 SHA Test Code. . . . . . . . . . . . . . . . . 153

B.4 Logic Allocation Data Structure Definition Header. . . . . . . . . . . . . . 154

B.5 Breakpoint Data Structure Header. . . . . . . . . . . . . . . . . 155

B.6 Serial Communications Source. . . . . . . . . . . . . 156 
B.7 Debugger Commands Header. . . . . . . . . . . . . . . . . . . 158

B.8 ICAP Access Routines Header. . . . . . . . . . . . . . . . . . 161

B.9 ICAP Access Routines Source. . . . . . . . . . . . . . . . . . . . 162

B.10 LLD Workstation Console Source. . . . . . . . . . . . . . . . . . . . 170

B.11 Main FPGA LLD Console. . . . . . . . . . . . . . . . . . . . . . . 184

B.12 Logic Allocation Lexer. . . . . . . . . . . . . . . . . . . . . . . 188

B.13 Logic Allocation Parser. . . . . . . . . . . . . . . . . . . . . . . 190

B.14 Programmable Debug Controller. . . . . . . . . . . . . . . . . . . . . . 194

B.15 Example of Generated Breakpoint Logic. . . . . . . . . . . . . . . . 200 


\section{Acronyms}

$\begin{array}{ll}\text { ABV } & \text { Assertion-Based Verification } \\ \text { API } & \text { Application Programming Interface } \\ \text { ASIC } & \text { Application Specific Integrated Circuit } \\ \text { BRAM } & \text { Block RAM } \\ \text { BSCAN } & \text { Boundary Scan } \\ \text { CFFT } & \text { Complex Fast Fourier Transform } \\ \text { DRC } & \text { Design Rule Checks } \\ \text { DSP } & \text { Digital Signal Processor } \\ \text { DUT } & \text { Device Under Test } \\ \text { EDA } & \text { Electronic Design Automation } \\ \text { EDIF } & \text { Electronic Data Interchange Format } \\ \text { FIFO } & \text { First In, First Out } \\ \text { FPGA } & \text { Field Programmable Gate Array } \\ \text { FSL } & \text { Fast Simplex Link } \\ \text { GSR } & \text { Global Set/Reset } \\ \text { GUI } & \text { Graphical User Interface } \\ \text { HAL } & \text { Hardware Abstraction Layer } \\ \text { HDL } & \text { Hardware Description Language } \\ \text { HLL } & \text { High-Level Language } \\ \text { HLS } & \text { High-Level Synthesis } \\ \text { HLV } & \text { High-Level Validation } \\ \text { IC } & \text { Integrated Circuit } \\ \text { ICAP } & \text { Internal Configuration Access Port } \\ \text { IDE } & \text { Integrated Development Environment } \\ \text { I/O } & \text { Input/Output } \\ \text { IP } & \text { Intellectual Property } \\ \text { JTAG } & \text { Joint Test Action Group } \\ & \end{array}$


LED Light Emitting Diode

LLD Low-Level Debug

MAC Media Access Control

NoC Network-on-Chip

OS Operating System

PAL Programmable Array Logic

PAR Place-and-Route

PATIS Partial module-producing, uutomatic, Timing-aware, Incremental, Speculative floorplanner

PDC Programmable Debug Controller

RAM Random Access Memory

ROM Read-Only Memory

RTL Register Transfer Level

RTR Runtime Reconfiguration

SHA-1 Secure Hash Algorithm

VHDL VHSIC Hardware Description Language

VHSIC Very High-Speed Integrated Circuit 


\section{Chapter 1}

\section{Introduction}

Digital system validation has reached a critical turning point. With the rate of new productions at an all time high due to a rapidly increasing consumer and commercial demand, the importance of an efficient and productive debug methodology has reached a tipping point. Time-to-market is a critical metric directly tied to production cost, however the increasing complexity and density of these systems works directly against the goal of improving efficiency and productivity.

In the last 20 years Field Programmable Gate Arrays (FPGA), a type of programmable logic device, have dramatically reduced design and validation times for developing complex digital systems. FPGAs give developers a near instantaneous means of prototyping and physically validating a digital design in real world conditions, when compared to the weeks-long wait required to manufacture an Application Specific Integrated Circuit (ASIC). However, even this level of rapid prototyping environment is not as flexible as needed. Build times for FPGAs may take days making even small changes a lengthy and unpleasant process.

In addition to long build times, FPGAs are notorious for the lack of visibility and control into the design, possibly the two most important attributes of validating and understanding any 
system. By their very nature, FPGAs are blank slates allowing any arbitrary, custom design to created. The excellent reputation they have earned in high-performance, small-area, and low-power industry sectors such as telecommunications, defense, and high-performance computing, as well as an effective prototyping technology for practically any sector highlights the necessity for increasing productivity and efficiency during validation and debug.

Several debug approaches are currently in widespread use. FPGA vendors, who are able to leverage their own knowledge of these proprietary devices overwhelmingly favor recreating the look and feel of an actual logic analyzer. To do so, special cores need to be configured and built into the design. Embedded logic analyzers internally record signal activity based on preselected criteria defined during the build phase and are physically integrated into the design. Third-party vendors, at a disadvantage by not being able to seamlessly integrate their products into the normal design flow, rely on add-on modules also integrated into the design, but also rely on aging interfaces that reduce the effectiveness, speed, and interactivity required for validation and debug.

FPGAs are unique in that they can reprogrammed an unlimited number of times, while some even allow one or more sub-regions of the configurable fabric to reconfigured independently from the rest of the device. This Runtime Reconfiguration (RTR) or Partial Reconfiguration (PR) capability has, to our knowledge, never been been leveraged for development-time methodologies. In addition, the ability to validate against the original software reference model, also suffers. Once hardware development begins, the reference model is physically isolated from the rest of the design process, and must be repeatedly and manually checked. The wealth of external interfaces and cores available on FPGAs allows some unique approaches for debug. Software allows a great amount of flexibility since it can be easily changed within a matter of minutes, and the availability of small, unobtrusive processors that can be embedded into a design afford interesting new approaches to design validation. 
If the perspective and context of debugging is changed from outside the FPGA to within, design validation and exploration are no longer limited in visibility, and the entire scope of all design registers are easily accessible. Software control of a design allows interesting approaches to exploration and inspection, and if coupled with a means of controlling execution, unprecedented perspective and insight into a working design is possible.

\subsection{Motivation}

Once the design is implemented and programmed into the FPGA, debugging or analyzing the design is difficult. There are no general purpose input/output facilities such as a keyboard or console as with a general-purpose computer, and no industry-wide standard targeted specifically for FPGAs. Current solutions are often borrowed from the ASIC industry, such as Joint Test Action Group (JTAG), which addresses a limited portion of the design. Manufacturer solutions, as well as the multitude of ad-hoc solutions also only allow a limited view of the design either spatially or temporally. The trade-off being that only a limited portion of the design can be viewed or a limited segment of the execution can be observed. Changing the perspective of this view is tightly coupled to the actual implementation and difficult to modify. The typical debugging solution is typically to instantiate cores within the design and record signal activity for later inspection. Changes to the perspective are treated like any change to the design and often require the same time-consuming implementations. In contrast, software development and debug environments allow complete visibility and control of the memory and execution space.

The frequent comparisons in productivity studies to software development environments are no coincidence. FPGA development environments and flows have been harshly judged against their software counterparts $[1,2]$. Software development environments are frequently 
cited as the gold standard for hardware development because of the speed and efficiency that they bring. This research aims to adopt several of these facilities for FPGAs, all based around the concepts of controllability, agility, and visibility of a design loaded and running on hardware.

\subsection{Problem Statement}

Typically, a hardware design is first modeled using a conventional high-level programming language, such as $\mathrm{C} / \mathrm{C}++$, Java or even a formal modeling framework such as Matlab. The development then proceeds by reconstructing a more concise design, this time specifically oriented towards the actual hardware. This is achieved by filling in implementation details unique to the platform such as specialized resources and device constraints. The highlevel reference model is algorithmic in nature, rather than cycle-accurate and contains no references to timing, which is elemental to hardware implementation. The transition from reference model to hardware implementation creates both a logical and physical gap in the designers understanding of the design space and the continuity of the design from model to reality. There are no failsafe automated solutions to converting the reference model to RTL, rather this is a manual process and the effort in validating the model is then repeated anew for the hardware design. The process of validating the original, vetted reference to hardware is a manual, unreliable, and error-prone approach. These separate flow processes are shown in Figure 1.1. It is noteworthy that not only is each phase of the development cycle almost entirely independent of the other, but also that no validation or debug techniques are applicable across any of the stages.

Furthermore, once implemented onto the FPGA, little meaningful and interactive interaction with the design is possible. There exists little to no abstraction between the physical 


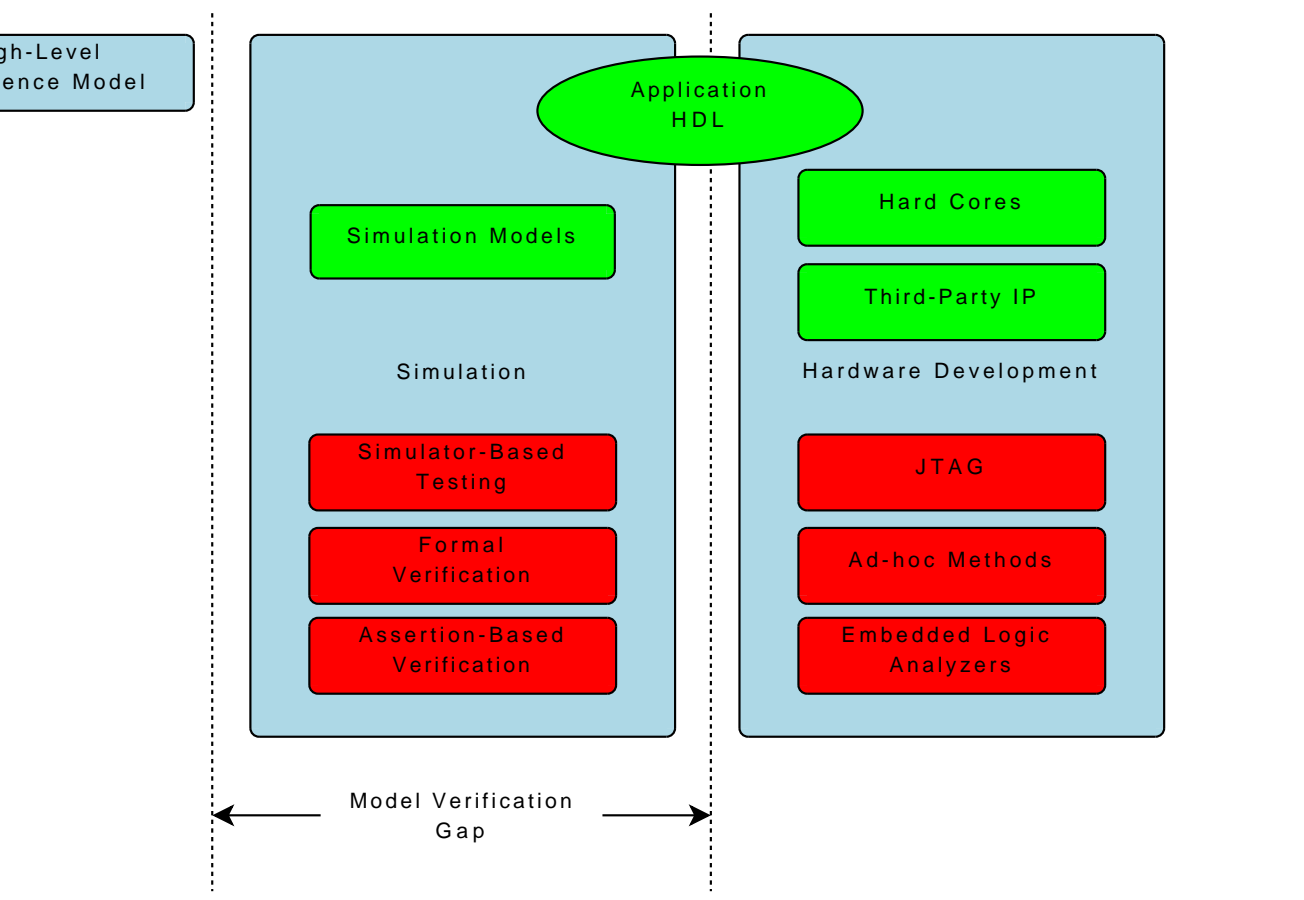

Figure 1.1: Hardware development flow.

implementation and the debugger's perspective of the design. Much like the development language, debugging is mostly a low-level task. Lengthy, error-prone implementations hamper FPGA productivity, as does limited visibility into the designs. Other than the primary inputs and outputs, the design is effectively sealed off from inspection. A cohesive and interactive control mechanism of a design is needed that allows both rapid and unlimited access to all aspects of the design.

\subsection{Contributions}

The contributions of this work are as follows:

- Development of a tool that addresses the model verification gap by raising the abstraction level for FPGA validation and debug. 
Debugging is traditionally a frustrating process since developers are responsible for closing the loop when validating synthesized hardware against earlier reference models. The customary view of hardware validation is at the RTL level, which when validating high-level functionality, is much too primitive. The developed system allows synthesized hardware and the implementation to be concurrently executed and compared at the target design speed on the same platform.

- Development of a tool that improves visibility, control, and agility for FPGA debug by introducing software development environment facilities.

Unlike software development, FPGAs have few means to inspect or debug a running design. The internal state elements of the design have limited or no visibility, and execution occurs at millions of clock cycles per second. An error may occur and by the time it manifests itself, the state of the device may have changed so much that its diagnosis is impossible. The developed framework brings some of the useful facilities found in software development environments to FPGAs. The system presented here allows a running, implemented design to be controlled and inspected at its target speed with the interface and agility of software development tools. While most FPGA development tools place the debugger's viewpoint outside the design, the developed framework places it within the FPGA. The presented work moves the designer's perspective inside the FPGA where its execution can be manipulated and its state explored from within, treating the FPGA itself as a random access memory.

\subsection{Organization}

The remainder of this dissertation is organized as follows:

\section{Chapter 2 Background}

This chapter provides background material on both hardware and software development 
to frame the perspective of the challenges faced in developing for FPGAs. Development and debug life cycles for FPGAs are explored, beginning from modeling and ending with simulation and hardware-based verification and validation. Commercial solutions, including the high-level language development techniques meant to mirror software development flows are also reviewed.

\section{Chapter 3 Related Work}

A survey of current debug-related work, both commercial and academic, is provided in this chapter. FPGAs are proprietary and the means of gaining insight into a design are limited and generally restricted to two methods: signal capture and JTAG. High-level synthesis is also discussed in this section since raising the abstraction of design entry to an algorithmic level also alleviates the need to do low-level analysis. Finally, a review of shortcomings of many of these methods looks to frame the presented research's contributions.

\section{Chapter 4 Improving Abstraction and Turnaround Time}

The model verification gap is analyzed to define the High-Level Validation framework. This framework aims to map untimed software models to implemented hardware designs by leveraging the wealth of information that can be extracted during hardware development through simulation. Rather than relying on simulation to validate, the proposed framework aims to move validation to hardware and tie its execution directly to the software model. Visibility, agility, and control for hardware-level debugging are then addressed by proposing a softwarestyle debugging environment for FPGAs. A method for implementing breakpoints as well as rapidly enabling, disabling, and altering them is also presented through the use of an on-board microprocessor.

\section{Chapter 5 Implementation}

In this chapter, the two debug and validation frameworks are implemented and benchmarked. First, the High-Level Validation framework is developed to non-invasively instrument and 
map a C-language reference model of an encryption core. The framework requires the development of a custom hardware peripheral to interface to the module to be tested. The system is shown to be capable of testing a hardware design executing at its intended target design speed. Finally, the Low-Level Debug framework is constructed, leveraging the Xilinx Runtime Reconfiguration flow. With this framework, debug scenarios can be modified and re-implemented into the hardware within minutes by isolating the debug breakpoint and toplevel modules each into their own separate reconfigurable region. Capabilities such as those found in software debugging environments, including breakpoints, stepping capabilities, and full immediate access to all design registers are demonstrated. The Low-Level Debug framework is demonstrated on three large designs where implementation time is prohibitively long. 


\section{Chapter 2}

\section{Background}

In this chapter, an overview of programmable logic and how it evolved into modern day Field Programmable Gate Arrays (FPGA) is presented, followed by a step-by-step discussion of the stages of development. The various methods of FPGA debugging and some of the challenges of each method are then presented. Next, high-level synthesis, an alternative to traditional hardware development languages that seeks to retarget conventional software programming languages for FPGAs, is presented. Finally, runtime and partial reconfiguration is explained.

\subsection{An Overview of Programmable Logic}

In the dawn of digital development, designers were required to physically build systems in order to test and validate them. Each wire of a design was individually connected to discrete logic chips, transistors, and other components. Often to improve visibility, colorful wires were used, each wrapped around small posts, lending this style of development its descriptive name wire wraps. The resulting rat's nest of wiring revealed the fragility and complexity of even a modestly-sized design. Frequently, finished products were just neater 
implementations of these prototypes, now committed to a manufactured component board with embedded traces. It would be some time before electronic design automation (EDA) tools established a more rapid and reliable means of prototyping designs while simultaneously introducing a new set of challenges.

FPGAs derive their name from their ability to be repeatedly programmed in the field or wherever they are installed, without the need to physically replace the device. Today, FPGAs are even more flexible and support the means of remotely re-programming the devices, further reducing the cost of ownership and maintenance. The gate array portion of the name refers to a historical and now purely logical organization of the array of logic gates that could be programmed to implement an arbitrary logic function, as shown in Figure 2.1. The gate arrays have since been replaced by a more efficient and compact mechanism. Modern FPGAs are still organized as arrays as seen in Figure 2.2, however specialized resources such as arithmetic blocks, memories, and processors are now interspersed among a vast sea of programmable logic elements.

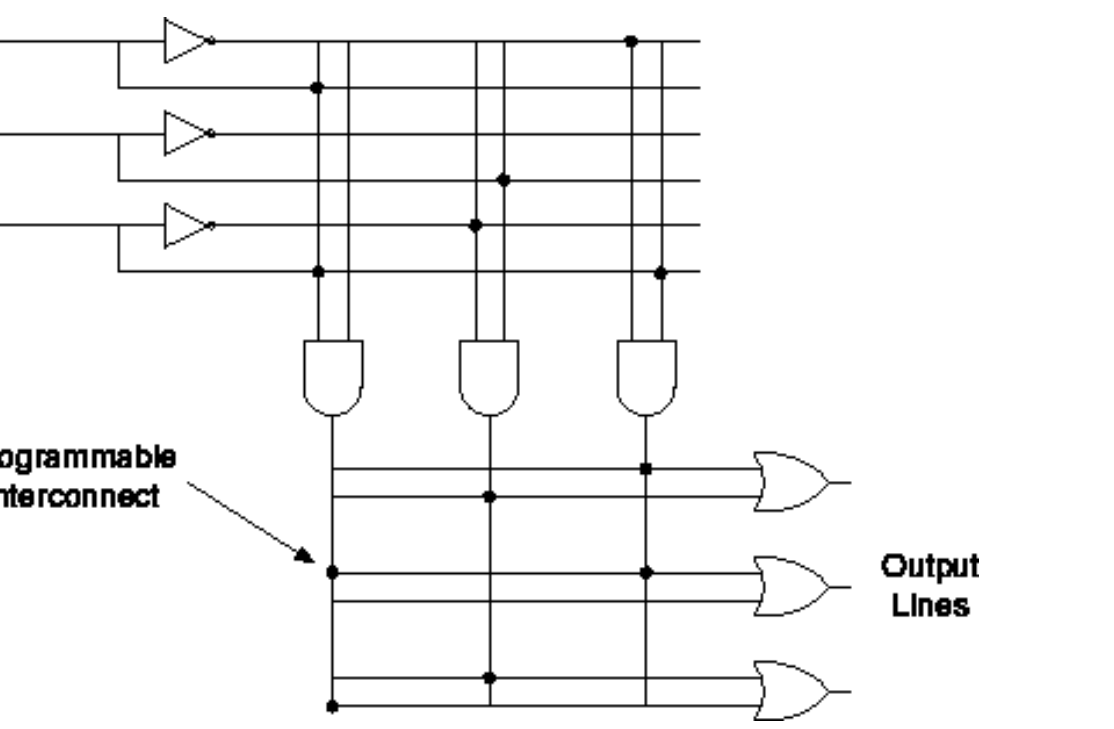

Figure 2.1: Early programmable logic devices with discrete gates connected by programmable interconnects. 


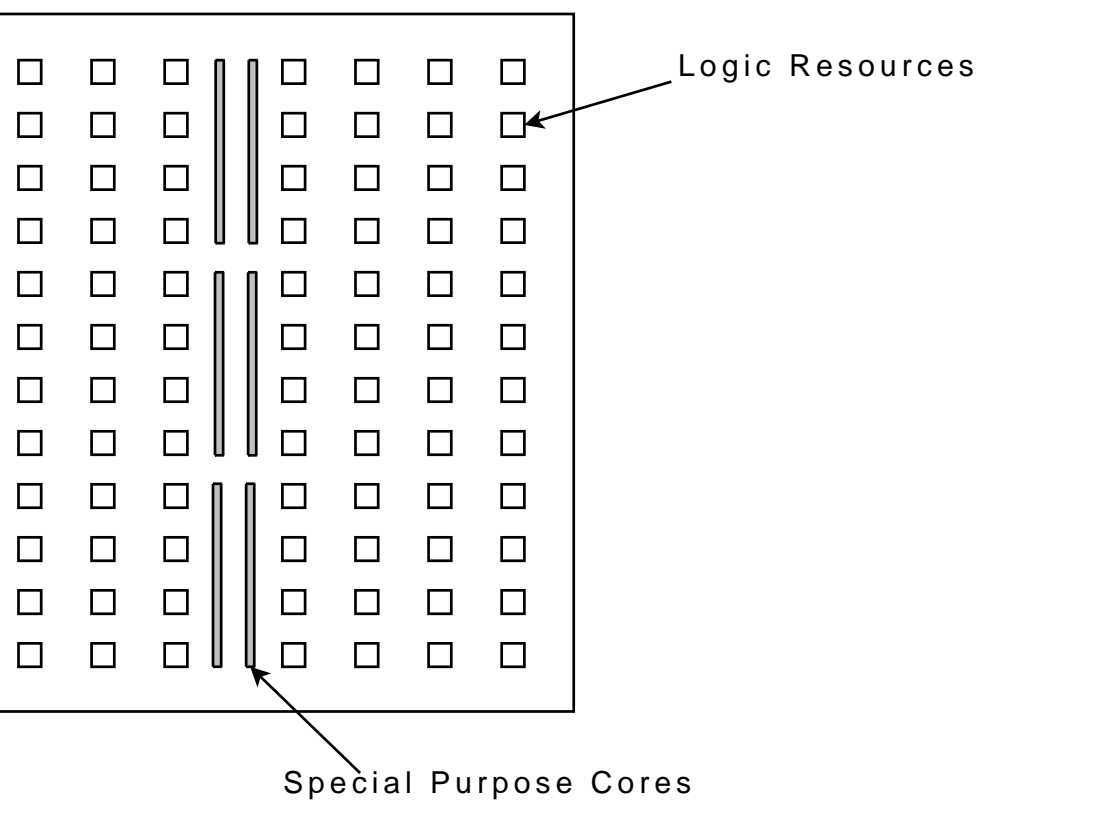

Figure 2.2: Modern FPGAs organized as a regular array of resources.

As Integrated Circuit (IC) technology advanced, multiple discrete components were combined into chips defining fundamental building blocks of digital systems, thereby allowing more complexity to be embedded into a smaller area. The need for a practical, repeatable, and efficient means of developing and prototyping complex designs became evident. Early FPGAs made their debut as precisely such an integrating technology allowing the interconnections and necessary logic (also referred to as glue logic) between these chips to be reconfigurable through software programming rather than physical wires. Manufacturers could now quickly deliver a product, placing the components on a board, all connected to an FPGA. The necessary connections and required logic could be experimented with and repeatedly revised, deferring the final design to even after units were installed. This also allowed revisions to be issued to previously sold and shipped products, which could then be updated on-site. Vendors who might not otherwise have had the financial means or number of forecasted products to justify an ASIC could still produce a viable and compelling 
product to compete in what was previously an exclusive market open only to the wealthiest companies.

Rather than be relegated to the ranks of a prototyping technology, FPGAs began to mature into their own distinct product line and became the focal point in the realm of highperformance industries once reserved for ASICs. Input/Output (I/O) speeds of FPGAs became competitively fast for real-world applications and the logic density - the equivalent number of physical logic gates - skyrocketed when innovative means of implementing and packing logic functionality into smaller areas was developed. One such innovation was the Lookup Table (LUT) [3] that eliminated the need to physically implement an array of logic gates and the means to programmatically connect them to realize an arbitrary logic function. Instead, the inputs of a logic function are replaced with address lines to a programmable read-only memory that returns the expected result of the original logic function when the corresponding lines are activated. As a result, the functionality of a LUT resembles that of the logic function's truth table. A conceptualization of this is shown in Figure 2.3. In reality, the actual implementation of a LUT is less intuitive but more compact. The vendor's EDA tools determine which signals to group as address lines, and compute the resulting output to be stored in the LUT's memory. This is far more space- and speed-efficient than trying to map a logic function to a statically built array of gates and determining the connections necessary in order to realize that function.

Advancing EDA tools and increasingly more powerful workstations advanced the state-ofthe-art, and as a result design processes became more productive. The advent of Hardware Description Languages (HDL) made the representation, modeling, simulation, and validation of hardware systems even more efficient. Whereas previously a digital system could not be validated without physically building and prototyping it with discrete components, validation could now be done within a simulation, quickly finding and fixing logic errors before building the design. 


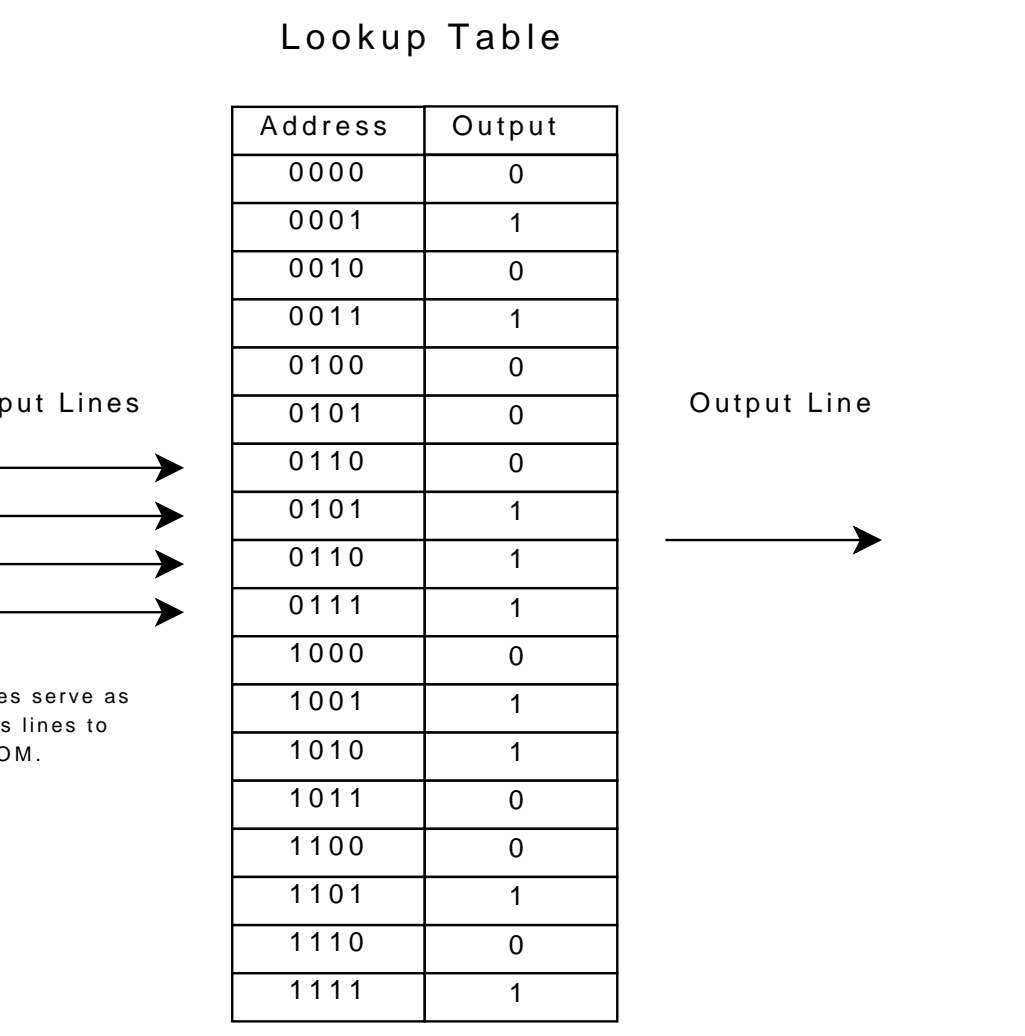

Figure 2.3: LUTs replace arbitrary logic with simple memories.

Figure 2.1, shows a simplified representation of one early implementation of a Programmable Array Logic (PAL) device, and the predecessor to modern FPGAs. The logic gates in this case-AND, OR, and inverter gates - are placed in a regular grid pattern and connected by programmable interconnects. The device is programmed by selectively enabling the programmable interconnects where crossing wires intersect, which when set create a route between a source and one or more sinks. Innovations such as LUTs obsoleted this architecture allowing more logic to be packed into an equivalent area. At the same time, smaller manufacturing processes also contributed to the increasing density and speed. A timeline of the densities of LUT-based architectures is shown in Table 2.1.

The data in Table 2.1 was compiled from Xilinx data sheets [4-12] of major FPGA releases between 1998 and 2010. For each release, the largest part was selected. When two companion families existed (typically manufacturers will build a more modest companion part that is 
Table 2.1: Timeline of logic density of Xilinx FPGAs.

\begin{tabular}{|l|l|l|}
\hline ReleaseYear & Device & $\begin{array}{l}\text { Number of Logic } \\
\text { Cells/LUTs }\end{array}$ \\
\hline 1997 & XC5215 & 1936 \\
\hline 1999 & XC40250XV & 20,102 \\
\hline 2000 & Virtex-II XC2V8000 & 93,184 \\
\hline 2002 & Virtex-II-Pro XC2VP100 & 99,216 \\
\hline 2004 & Virtex-4 XC4VLX200 & 200,448 \\
\hline 2006 & Virtex-5 XC5VLX330 & 207,360 \\
\hline 2009 & Virtex-6 XC6VLX760 & 758,784 \\
\hline 2010 & Virtex-7 XC7V2000T & $1.955 \mathrm{M}$ \\
\hline
\end{tabular}

smaller in size, consumes less power, and subsequently less expensive), the larger family was selected. Logic cells were chosen as the comparison metric because it appeared across all devices, albeit under different names. One logic cell equates to one LUT (the word LUT does not appear until the release of the 4000-series in 1999). Logic cells were also chosen because this is the smallest, most primitive user-programmable structure in the device and from where FPGAs derive their core functionality. The number of inputs to the LUT also increased during this time period, further compressing the amount of logic that could be packed into a single logic cell. Logic capabilities increased rapidly yet as shown in Chapter 3 , methods of working with the devices has remained relatively unchanged.

It should be noted that there is no one clear metric by which to truly measure FPGA density. Vendors frequently change terminology, restructure the architectures, and emphasize different parameters in the product literature. While earlier literature focused on the number of "equivalent logic gates" - the equivalent number of primitive Boolean logic gates that the device could implement - this terminology quickly fell out of favor. Later releases would also cite of the "maximum number of gates" - another ambiguous and short-lived parameter - which also similarly increased over the same period. The effectiveness of attempting to measure logic density is further confused when hard macros or hard cores are 
considered. Macros are discrete components physically built into the FPGA, typically complex or frequently utilized cores that are useful across a large number of designs. Requiring designers to implement their own cores promotes poor design practices, reduces re-use, forces high levels of design specialization for a particular architecture which makes it difficult to port to future releases while at the same time wasting valuable real estate in the reconfigurable fabric. Common hard macros include memories, signal processing cores, multipliers, communications cores such as Ethernet MACs, and even microprocessors (dual PowerPCs were available on the Xilinx Virtex II-Pro). The impact of including such cores is dramatic when the number of resources freed and the potential for increased performance and power efficiency are considered.

FPGAs are not only expensive, but difficult to program as well. Unlike conventional computers, programmable logic design has not had its renaissance. Whereas higher level programming languages such as C or FORTRAN eventually obsoleted the use of assembly-level languages ushering in an era of innovation, progress, and accessibility [13], the complexity of FPGAs and their chief purpose - to accelerate computation well beyond the capabilities of conventional architectures - make these obstacles unlikely to be resolved for some time. FPGAs are programmed using low-level, primitive languages which describe the transfer and transformation of signals between registers. Higher levels of abstraction are difficult to obtain.

Despite the many drawbacks, FPGAs are popular in the communications, industrial, and military sectors. FPGAs are the primary processing power for Internet router giant Cisco's flagship products. FPGAs are frequently deployed in aircraft, satellites, and other vehicles where lots of processing power is needed but space and power are limited. FPGAs are particularly attractive for satellites since the application can be remotely changed, allowing the satellite to be dynamically retasked while in orbit to changing mission requirements. 
Due to the cost and difficulty in development, FPGAs are not typically found in consumer electronics, however vendors are now producing a family targeted specifically towards the automotive industry's information and entertainment ("infotainment") consoles found in high-end vehicles. German automakers BMW and Mercedes have outfitted their high-end sedans with FPGA-powered video processing equipment [14] such as night-time, low-light, or rear-facing assistive technologies; other auto manufacturers are following suit by replacing certain on-board systems with FPGAs.

FPGAs currently operate at a comparatively lethargic clock rate, yet can outperform conventional systems running at an order of magnitude higher clock speed. In the last decade, operating frequencies have risen from sub-100 MHz to around $500 \mathrm{MHz}$ clock rates in the premium speed-grades. In comparison, commodity processors such as those from Intel and AMD achieved the $1 \mathrm{GHz}$ milestone in 2000 [15]. Yet, FPGAs remain as one of the few viable means of accelerating streaming applications, and performance may exceed that of even high-performance commodity processors. The explanation for this discrepancy is in the flexibility of the FPGA's unique architecture.

FPGAs do not carry the customary operational overhead of conventional computers; they are not general-purpose processors that have a single or limited thread of execution and a fixed instruction set. Yet neither are FPGAs natively multi-process capable as are most modern microprocessors. Multi-processing is a clever slight-of-hand that gives the appearance of simultaneously processing several unrelated tasks, when in fact the processor is only physically capable of servicing one process at a time. Multi-processing capability allows the processor to quickly and transparently change processes either after a predetermined time interval or when a higher priority task has preempted the current task. The generalpurpose, fixed-instruction set architecture and multi-process capabilities of microprocessors make them difficult if not impossible to target to specialized, high-performance applications, 
particularly those that process continuous streams of data. In addition, operating systems (OS), specifically user-friendly consumer OSes, are busy servicing device driver requests, controlling peripherals such as network interfaces or hard drives, and managing memory allocation to improve user experience.

Dramatic performance gains achieved with FPGAs are possible through the flexible, reconfigurable architecture. Unlike conventional processors and associated software tools that serialize a set of predefined instructions to perform a task, FPGAs have no such restrictions. Instead, FPGA developers can custom design an accelerator to include as many specialized pipelines or processors as desired. FPGA applications are referred to as a custom processors, hardware accelerators, co-processors, or any number of similar names. With a custom design such as this, performance can dramatically improve. The inclusion of high-performance, specialized cores has increased the capabilities of FPGAs to enter many more industry sectors rather than simply serving as glue logic.

Despite the great potential of FPGAs, the development process is not as streamlined as it is for software development. In contrast, the growing prominence of software engineering has heightened structured software architecture and reuse, while the same is rarely true or even possible with hardware development. The primary culprit is the uniqueness and variations of subsequent architectures and how tightly-coupled a design becomes to the target device, and as explained next, the complex development process.

\section{$2.2 \quad$ FPGA Development}

FPGA development typically begins with a functional, high-level language (HLL) model that is used throughout the design cycle as a reference for both simulation and hardware development. HLLs provide access to elegant data structures, complex control constructs, 
and resources such as file systems and debuggers that enable rapid algorithmic development and extensive testing, yet are not burdened with timing and synchronization details.

Once the high-level model has been validated against the original specification, HDLs such as VHDL or Verilog are used to develop cycle-accurate simulation models targeting a specific hardware platform. HDLs are low-level languages used to describe the transfer of signals between registers and characterize the concurrent nature of a hardware design. Simulation models specify the register-level transfer and processing of data, bus-widths and control signals that were not defined in the functional reference model. Most device families contain specialized, high-performance cores, such as Digital Signal Processing (DSP) cores and memories that are common in designs. These can be instantiated into a user design like any other component, however their interfaces, behavior, or configuration are frequently specialized for the specific architecture and are subject to change in subsequent architectures.

With some effort, simulation models may be validated against the reference design, but this is often of limited usefulness. Simulation models for Intellectual Property (IP) and hardware modules are often themselves non-synthesizable behavioral HDL models optimized for simulation or obfuscated to protect the IP. These may inadvertently differ from the actual implementations. As a result, the model being simulated will not accurately reflect the synthesized hardware version. Additionally, simulators silently accept legal but physically impossible coding practices for FPGAs. Optimizing synthesizers may misinterpret a developer's intent and alter the design producing unexpected results, whereas synthesis pragmas are intended to produce alternate versions in synthesis [16]. There are a handful of solutions available to debug the resulting physical design, none of which offer the rapid turnaround and full visibility found in software development.

On the other hand, software developers have created generations of tools to rapidly validate and debug their applications. Build tools such as Make [17] analyze source code dependencies 
and selectively rebuild only the necessary dependencies. Linkers and library archives preserve object independence until the final static linkage or, as in the case of dynamic linkage, until runtime. Small revisions can quickly be made to an application and tested directly on the target platform, usually in a matter of minutes for even complex applications. Debuggers and profilers offer extensive visibility into an executing application and allow complex breakpoints to be specified and altered without recompiling. Figure 2.4 shows the software compilation flow. Software compilation units remain as independent intermediate objects even after being compiled and integrated into a library archive until linkage or runtime, and as such are easily revised. No such development model is readily available for FPGAs.

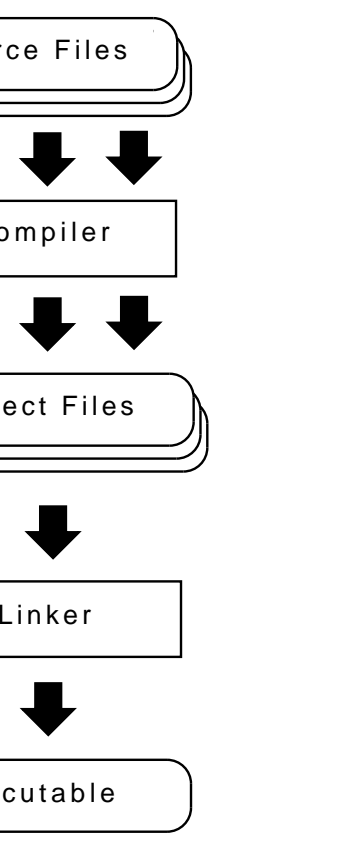

Figure 2.4: Overview of software development flow.

This flow is in stark contrast to the traditional FPGA flow. In software compilation, a relatively short time is required and high level of parallelism is possible in producing intermediate files. The intermediate object files remain independent even within their destination library archive, and are easily individually replaced. However, in FPGA design flows the separate components of the design are combined into a monolithic format during the early stages of 
compilation, typically following synthesis. A basic depiction of the FPGA flow is shown in Figure 2.5.

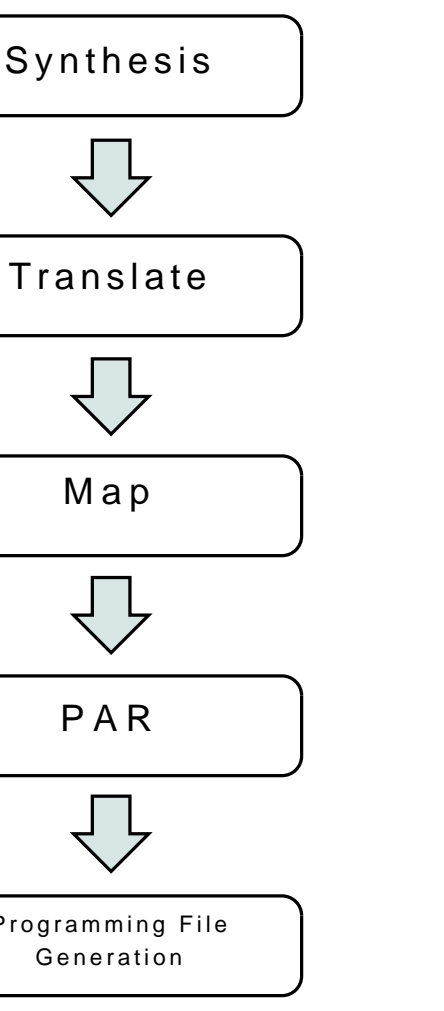

Figure 2.5: Simplified view of FPGA development flow.

Unlike software, FPGA development flow is serial. While individual modules may be synthesized in parallel, the rest of the flow aggregates all the individual components into a singular monolith and operates on the entire design globally. Unlike software, many operations can have a global impact on the rest of the design. For instance, logic optimizations or reductions can have a ripple-effect that emanate beyond module boundaries and throughout the entire design. Place-and-Route must find an optimum solution that locates components where they can be successfully connected using the FPGA's complex routing architectures. This is a challenging problem in that there may be a limited number of routes with acceptable latencies. At that stage, large designs are fragile and changes to accommodate routing or 
timing can be disruptive causing a series of changes. A thorough discussion of each of the stages is given in Section 2.2.1.

Vendors are aware of the time burden associated with FPGA development and have attempted to address these issues. Modular and incremental flows for FPGAs, ideas borrowed directly from ASIC development, attempt to compartmentalize designs and avoid lengthy re-implementations $[18,19]$. However these flows require that floorplans and interfaces be stable. This is a difficult prerequisite to meet especially in the early stages of development where the design may be in a constant state of flux. Even with modular and incremental flows, a seemingly small change may still require a full re-implementation. The PATIS project [20] discussed in Section 4.3.1 addresses this problem.

\subsubsection{Design Flow Overview}

The FPGA development flow is an elaborate multi-stage process. Vendors attempt to mask the complexity by providing applications that automatically generate scripts or interfaces for specialized cores, or graphical, user-friendly applications to control and monitor the entire build process. Yet, there remains a great deal of device- and design-specific information that must be known and applied at each step. Originally, the tool flow was developed to be a definitively layered sequence, with each stage performing a distinct task while deferring the low-level, device-specific implementations for later stages. However for optimization purposes, device-specific information is now included at every stage and the layers have lost their well-defined boundaries. Typically, an application will provide suggestions or attempt a preliminary effort for subsequent stages enabling iterative executions between the two without user intervention. Proprietary file formats also have evolved to include much of the meta-information produced in earlier stages, reducing the number and complexity of 
command-line switches that needed to be passed along to different stages. These are subtle, yet welcome improvements.

FPGA flow consists of five steps: synthesis, translation, mapping, place-and-route, and finally programming file generation. Vendors have not adopted a universal terminology, but the purpose of each stage is consistent across different vendor's flows. A general diagram outlining the flow between stages is shown in Figure 2.6 with an overview of each of the stages following.

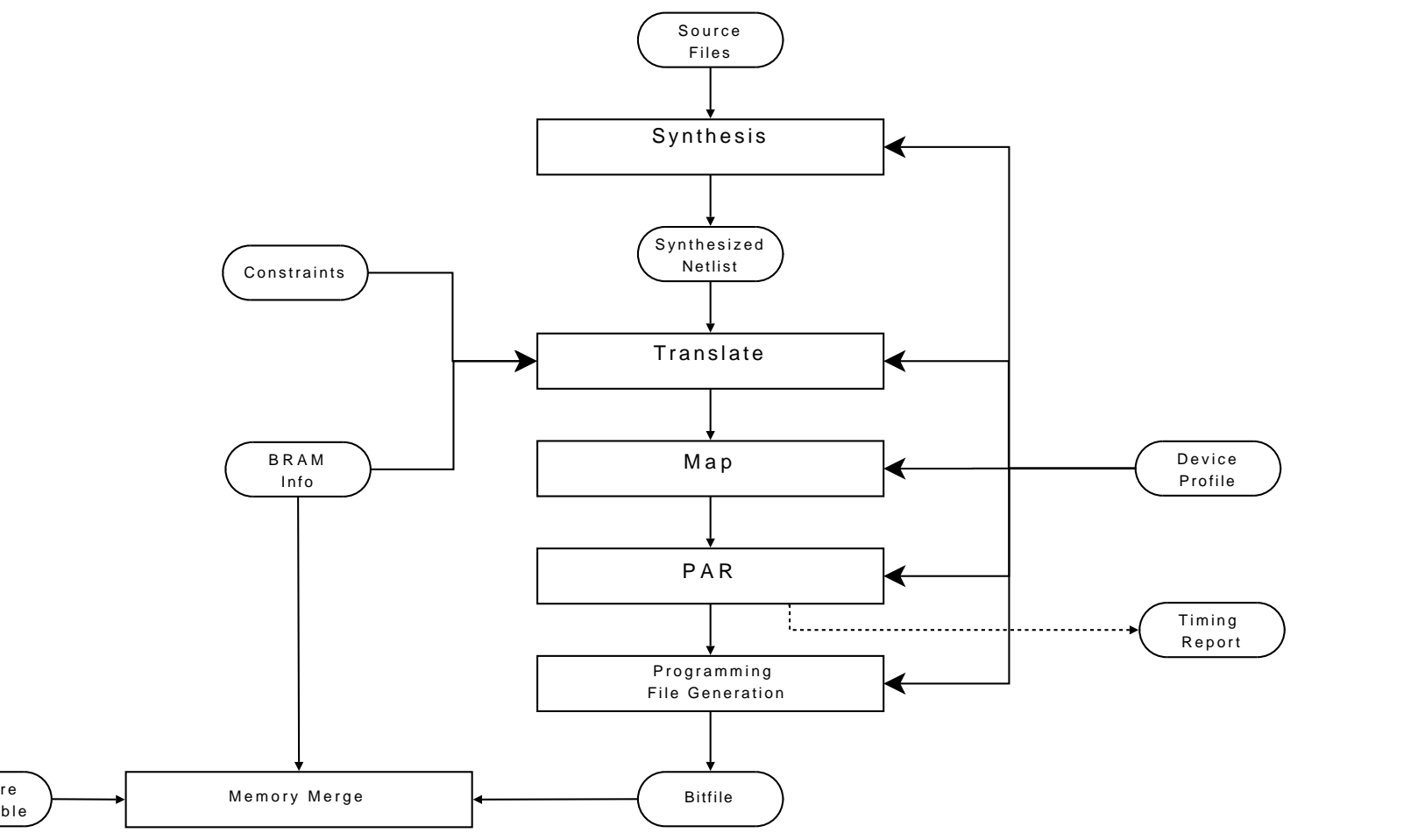

Figure 2.6: Detailed overview of FPGA development flow.

\section{Synthesis}

During synthesis, the original HDL is converted to a structural netlist. The structural netlist, also referred to as simply a netlist, consists of elements in the design and their connections. HDL can be written as a behavioral specification, which raises the layer of abstraction 
to higher-level constructs (including control flow, clock events, and some basic arithmetic operations) or as structural HDL which defines a more primitive, precise but less intuitive definition of the design. Netlists are purely structural in makeup, defining the elements and their interconnects of the design without any high-level elements or language representations. Rather, only primitives, the most basic elements of digital design such as Boolean gates, or physical structures found on the FPGA are found in netlists. The synthesizer is one of the few applications for FPGA development that can be written by independent third parties. However, users are rewarded for using the vendor's synthesis tool since these are typically tightly integrated with the rest of the tool flow.

In the past, synthesis was device-independent. However the increasing number of highperformance and device-specific cores found on FPGAs which can be explicitly instantiated into the design forces the synthesizer to possess some knowledge of the device. Also, being the first stage of the development process, the synthesizer is in the unique position to analyze the original source including pragmas written by the original author and perform preliminary mapping efforts based on design patterns found in the original HDL. Synthesizers rely heavily on hard-coded HDL design patterns that it uses to recognize and build optimized code or instantiate hard macros for the target architecture. The process of identifying and producing specific design elements is referred to as inference. A core is inferred when the synthesizer recognizes the predefined HDL pattern, such as a memory, state machine, shifter, or arithmetic core and then instantiates an optimized interface for that pattern. If the synthesizer is not able to infer the pattern correctly such as from a non-standard implementation, the component will not be built in the most optimized manner and placed into one of the FPGA's physical cores, or may not operate as expected. For example, memory inference fails when specified in capacities other than powers-of-two. The list of inferences and their corresponding HDL patterns are listed in the synthesizer's documentation. An example of one 


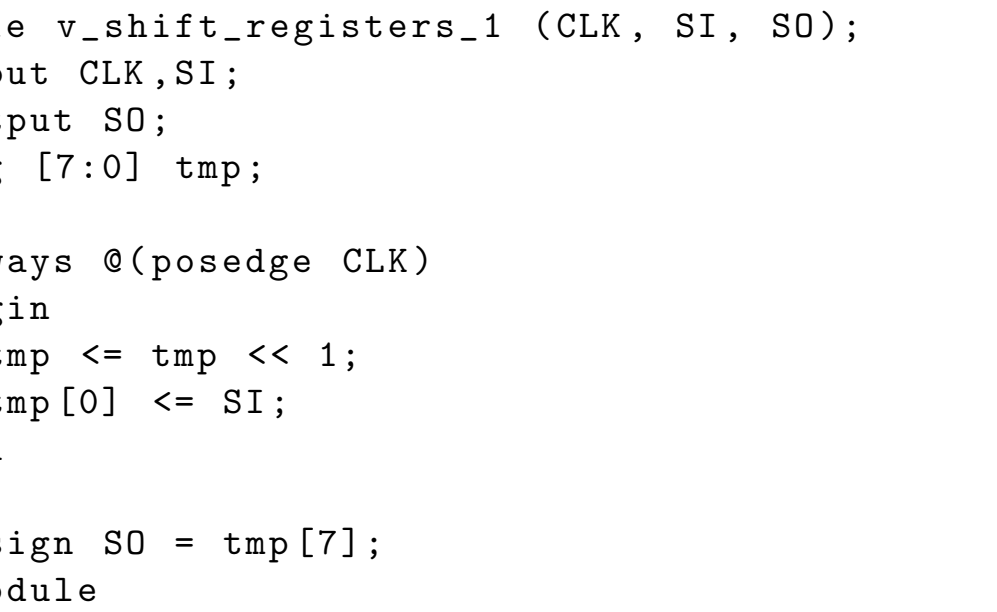

Listing 2.1: Verilog shift register definition from XST synthesizer documentation.

such pattern for a Verilog shift register, taken directly from the documentation of Xilinx's XST synthesizer [21], is shown in Listing 2.1.

There are several advantages to inference. First, common components of a design can be created in a structured, repeatable manner without the need to explicitly instantiate devicespecific components. This promotes some level of platform-independence as well as reusability. Second, the user or the tools can allocate inferred instances across available resources for the device or if necessary implement them in logic if there are not a sufficient number of cores available. For optimization purposes, the synthesizer needs to know architectural details of the target device to determine if there are a sufficient number of cores available, either through instantiation or inference. However, the synthesizer does not definitively allocate or assign resources. The final assignment is done later once the entire design's resource requirements have been resolved. The design can also be entered schematically and processed by the synthesizer, however in practice this is not as common as HDL. The synthesizer produces a structural netlist, either in the industry-compliant Electronic Data Interchange Format (EDIF) or the vendor's proprietary binary format. The advantage of 
using the vendor-specific format is that additional information may be passed within the files to subsequent tools of the flow, reducing the burden on the developer. Synthesis occurs relatively quickly (on an order of seconds at best) depending on the size and complexity of the module. Modules may also be separately synthesized, providing some separation from the rest of the build process.

\section{Translation}

Once the synthesizer has produced a netlist, the design must then be translated. During translation, also referred to as building, netlist components produced during synthesis are further reduced to primitive logic gates. Device-specific cores instantiated in the original HDL are also not processed during this phase. This is the last stage of the flow where nonproprietary or industry-wide input files are accepted. The rest of the flow occurs entirely within proprietary file formats and must be handled using the vendor's tools. Along with the device information, user constraints are also provided to the build tool. User constraints are specifications such as physical pin assignments, timing constraints, and memory parameters for the proper operation of the design that the tools cannot infer on their own. Pin assignments map the device's physical external pins to the design's top-level module input and output ports. Timing constraints specify timing and signal-related parameters, typically for interfaces with strict timing requirements such as Ethernet MACs, high-speed I/O interfaces, or the design's clocks. Timing is not significant for translation and is principally used once the design's connections have been routed (during place-and-route) to ensure that the design will meet timing. Since the tools are proprietary, it is not definitively known to what extent timing is addressed at this stage, but it is most likely that they are integrated into the design databases, analyzed for preliminary design rule enforcement, and referenced for mapping optimizations. 


\section{Mapping}

During the next phase, the mapper will logically map components produced during translation onto the target device. The mapper does not physically map elements to a specific core, but logically identifies candidate target core types. It is during this phase that the abstraction benefits of inference are realized. The mapper can now definitively determine which, if any instantiations must be implemented as logic, rather than the preferred core assignment strategy. For example, a design with more RAMs than the available physical memory cores will require some of those RAMs to be implemented as distributed RAMs. The distributed specifier indicates that the component will built using the FPGA's logic blocks rather than be instantiated to a physical, special-purpose core. Decisions during this and later stages have the potential to create a ripple-effect of complications. If an instance must be implemented as distributed, more logic is now required than originally assumed. Tightly interconnected cores such as memories require that all the corresponding logic be placed in close proximity to one another in order to meet timing requirements. As the design components of the design have no physical locations assigned at this time, the mapper has no way of determining if the final design will fit on the part, if there are sufficient routing resources available, or if the final routing will meet timing. These issues are addressed during the next Place-and-Route phase. Mapping handles optimizations such as constant folding where a constant signal makes logic in its path unnecessary. For instance, a constant zero signal feeding an AND gate will make the gate unnecessary as its output will in turn always be a constant zero. This gate can be removed without affecting the operation of the design. Optimizations such as these may free up resources, making it possible to pack even more logic into a smaller area which in turn may make routing and timing closure easier. Timing closure, which is addressed during Place-and-Route, is achieved if components are both placed and connected ("routed") in such a way that the signal's latency does not violate the 
timing constraints. For example, a signal whose latency along a path exceeds that of of the clock period violates timing or does not meet timing.

\section{Place-and-Route}

In Place-and-Route (PAR), the components that were previously logically mapped onto the FPGA's resources are now physically placed and the wires routed between them. Conventionally, the Map and PAR stages were distinct, however current tool implementations will perform a preliminary PAR during the map stage and reiterate mapping if required. Without this optimization, mapping would need to be rerun manually if PAR fails. Placement and more specifically routing are very computationally and memory intense. At this stage, precise models of both the FPGA and the design must be built and stored in memory. The algorithms used during PAR rely heavily on traversing these structures, and as such require many non-adjacent memory accesses which quickly overrun caches. PAR is the most timeconsuming stage of the build process, and is the stage most likely to fail for complex designs with high device utilization.

Meeting timing requirements are also the responsibility of PAR. A poorly placed design stresses the router which must find an efficient route - out of a vast number of possibilitiesto meet timing. Further complicating PAR is that routing is not a simple distance-dependent metric. That is, placing two components physically close to one another does not guarantee that a low-latency route exists between the two. FPGAs have complex, segment-based routing architectures where wires are categorized as local or long-distance, spanning one or multiple segments before reaching a programmable interconnect where it is patched to another segment. The individual segments, along with the programmable interconnects which add latency, do not have simple timing models. Furthermore, routes may be congested resulting in a circuitous path to connect two components. The best placement of a component 
for timing purposes may be non-intuitive, such as physically distant location where there are fewer, but physically longer routing segments to the destination. Counter-intuitively, this can improve timing. As such, PAR is a lengthy and iterative process. For large designs utilizing a high percentage of the FPGA, PAR times exceeding a day are not uncommon. Timing report generation is a separate procedure from PAR and is useful to determine details of which parts of the design are causing timing to fail. Often, a different design technique such as pipelining an operation alleviate timing failures. A successfully placed and routed design is ready to be committed to a programming file and loaded onto an FPGA.

\section{Programming File Generation}

Finally, the actual file that will be loaded into the FPGA is produced during programming file generation. The bitfile, programming file, configuration file, or bitstream is produced by interpreting the final placed and routed design into a binary file comprised of a stream of bits which configure the FPGA's resources. It is common in this stage for the generation application to perform a final design check ensuring there are no conflicting programming options, such as a conflict that might physically damage the device. A header is prepended to the programming file that contains special instructions for the loader and may activate special features on the FPGA, such as security. The FPGA is ready to be programmed with a special programming cable and loader application which either directly programs the FPGA or commits the design to an on-board FLASH memory or other external storage.

If the design contains RAM blocks, the contents can now be initialized. If RAMs were specified early in the design and build process, a logical mapping of design elements to memory elements would have been produced. This mapping is initially used during the translate phase, but is again referenced to prepopulate the RAMs before configuration so that memories are not empty at power up. This is particularly relevant if the design contains a 
microprocessor. Without this capability, processors would need temporary boot up sequences to hold them in a legitimate state until the RAMs are populated or require firmware to load programs from another source, such as FLASH or external disks. ROMs on the other hand, are typically initialized during synthesis by specifying their contents either in the HDL or as an external file that the synthesizer processes and incorporates into the netlist.

\subsection{Debug Methodologies}

For FPGA design debugging, there are a limited number of strategies. FPGAs are proprietary devices, which limits third party products in this area. Unlike conventional, commodity computers, FPGAs have no standardized architecture or discernible platform like an operating system or even basic input/output by which to interact. Debugging generally requires intimate knowledge of the device and the design. Improvised solutions are common, such as activating diagnostic LEDs when certain events occur. This requires an intimate knowledge of the design and is a lengthy process of progressively interpolating to the cause and re-implementing the design each time. External logic analyzers can be effective, but require signals of interest to be routed to external physical pins. This can interfere with design pin assignments and affect timing closure. If the signal is within several layers of modules, each enclosing module will need its interface modified in order to route the signal to the external pin. Once finished, the interfaces must be reverted and the design re-implemented anew. Lengthy implementation times, especially during place-and-route make these ineffective strategies.

In the creation of large or complex designs, a high-level language reference model is often first created to develop and validate the algorithm. This reference model becomes isolated from the remaining development cycles due to language and technological incompatibilities. 
Subsequent development in HDLs re-implements the algorithm anew, focusing on data flow, extracting parallelism, timing, and control signal interaction - aspects not captured in the reference model. After simulation, the HDL design may be adapted for synthesis. Though the same HDL may be used for both simulation and synthesis, this is not always the case. Thirdparty IP may be implemented differently for simulation and synthesis for faster simulation and optimized for the target platform. Simulation models may be obfuscated or encrypted to protect intellectual property. Unintentional variations between the models may exist or an optimizing synthesizer may misinterpret designer intentions, providing unreliable or unpredictable results during development [16]. The resulting gap between HLL models and synthesizable hardware is the model verification gap, illustrated in Figure 2.7.

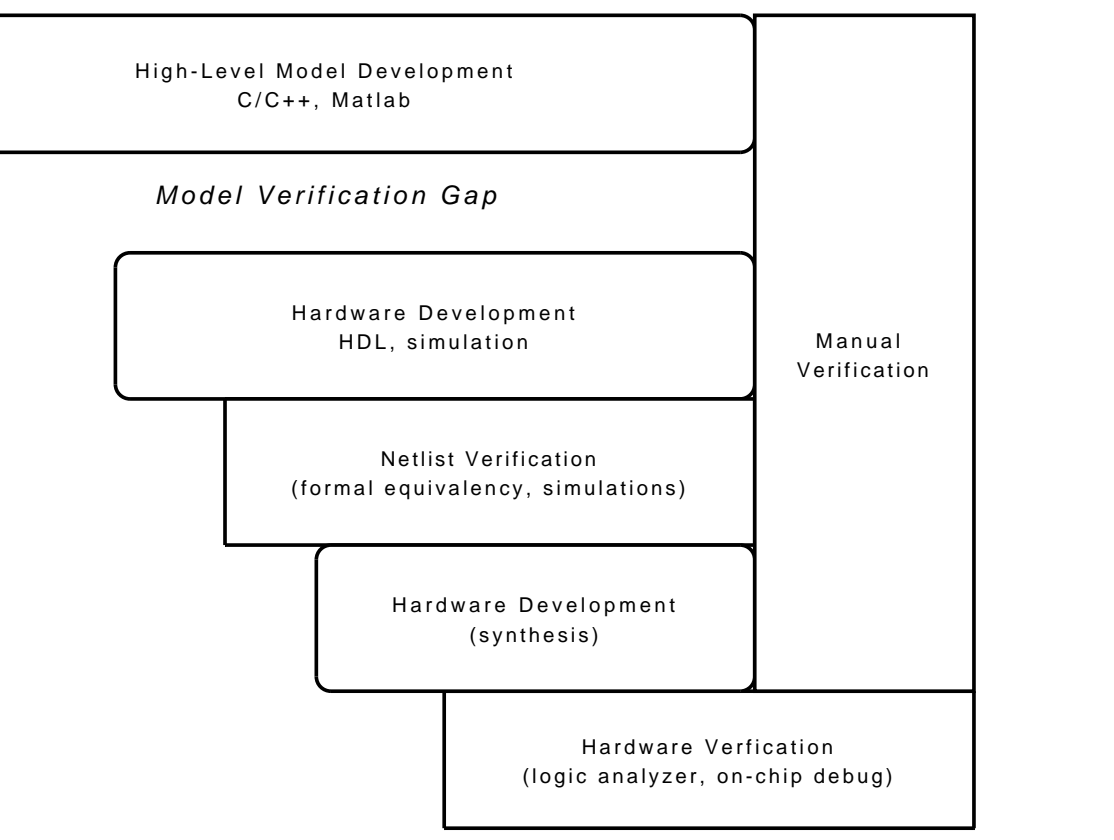

Figure 2.7: The model verification gap in hardware development.

The model verification gap is a significant shortcoming in hardware development. The design validation team must develop ad-hoc solutions to bridging it, or bridge it manually. Either way, the effectiveness and productivity of validating against the original reference model is limited and largely targeted towards the simulation model. No tools are known to exist 
that allow the original HLL reference model to be directly linked to the synthesized and implemented hardware.

Designers have long relied on many of the same debugging methods, many of which are complex and difficult to adapt to different scenarios. As designs have increased in size and complexity, tools have lagged in the ability to adequately handle these demands. The size of FPGAs has increased so dramatically in recent years; 32-bit processor based workstations are now incapable of targeting these larger parts while 64-bit processors are now the norm [22]. Visibility into a design programmed onto an FPGA is limited, and some types of design errors such as timing errors, can be elusive across design turns. Even worse, the synthesized version of a design can differ from what was prototyped in simulation. Unlike software designers who can frequently develop and test on their target environment, hardware developers do not have the same visibility.

Third-party IP, which addresses the growing interest in modular design and reuse long practiced in software development, also presents its own challenges. A vendor may develop several models of an IP core: one optimized for fast simulation using high-level, non-synthesizable behavioral constructs and another device-optimized version for synthesis. Often these models differ. Pragmas (suggestions that the designer can provide to the tools) placed in the code to guide the synthesizer or enable special conditions during simulation may also complicate the crossover from simulation to synthesis. The result is a design that performs differently in simulation than it does on the hardware [16].

\subsubsection{Custom Methods}

Developers frequently employ improvised solutions while debugging. One such prevalent practice is to activate diagnostic LEDs commonly found on development boards when certain conditions or events occur. Developers will iteratively place triggers throughout the design to 
illuminate an LED when certain areas of logic are active or desired conditions have been met. This technique is carried out by iteratively placing activating logic around areas believed to be problematic and interpolating to a likely cause of failure. An intimate knowledge of the circuit and often the device is required. If a shared memory or on-board processor is present, a developer may develop custom programs to inspect those memory locations. External logic analyzers are also used, but require signals of interest to be routed to external physical pins. This sometimes interferes with design pin assignments and can affect timing closure.

The drawback to these techniques is that any change triggers a full and lengthy rebuild of the entire design, which in some cases can be an entire day. The worst and all too common scenario is that several days may pass with no appreciable insight into the cause of the error.

\subsubsection{Simulator-Based Development}

Simulators are a standard tool available to developers, but have a rapidly diminishing rate of return of usefulness if too much time or effort is invested in the simulation model. Simulators are useful in developing custom logic, yet integrating with third-party IP or proprietary vendor cores can be problematic. To do so, developers rely on vendors to provide an accurate simulation model of their core, which is not always the case [16]. In some scenarios, such as with I/O or device configuration cores, no simulation model is feasible. An example particularly relevant to this research is the Internal Configuration Access Port (ICAP) core available on Xilinx FPGAs. The ICAP allows direct internal access to the configuration registers of the FPGA and enables a form of introspection on the configuration registers or more commonly allows the design logic to trigger and perform a reconfiguration of a portion of the FPGA while it is running. Since the ICAP relies on a configured FPGA and its fabric, a simulation model is unfeasible. This makes the ICAP a very difficult target for developers and requires multiple development turns. 
Simulators make all signals and registers in the design readily available and have intuitive user interfaces where waveforms can be arranged and compared. Signal transitions or values can be searched for, breakpoints can be set, and results saved for later analysis or regression testing. In contrast, the same level of flexibility is available to software developers directly on the target platform.

Newcomers to digital design quickly learn that even though a design appears to work well in simulation, it may not behave as expected once implemented on the FPGA. While it is common for software developers to debug directly on the target hardware, to do the same is difficult in hardware development. Simulators are a convenient development environment, but one that can be overused since they provide no guarantee of accuracy. This mismatch is further compounded by hardware languages which allow practices that are legal in simulation, but impossible to implement in hardware. Optimizing synthesizers may misinterpret a designer's intention and implement the logic differently or even strip it from the design altogether [16]. Additionally, fine-grain simulation can be very costly in terms of time and resource requirements. Extremely large and complex designs, such as processors, may take days to accurately simulate even a single minute of operation.

\subsubsection{Commercial Debug Solutions}

The proprietary nature of FPGAs places the burden of creating the highly specialized tools necessary to debug them primarily on the manufacturer. As such, there are a limited number of third-party commercial tools that enable debugging directly on FPGAs, with the vendor's own tools being the most frequently used. Of those, embedded logic analyzers (ELA) are the most common class of debug tool, most often implemented as cores that are instantiated as part of the design with the results viewed through a software application with the look and 
feel of an actual physical logic analyzer. Third party debug tools are often external state analysis applications.

When using embedded logic analyzers, designs may eventually accommodate or adapt to their presence and when removed create timing or routing anomalies that are difficult to trace and necessitate a new series of fixes. Frequently, this requires full re-implementation of the design. Routing and logic resources become a concern as wires are physically routed for each monitored signal and the cores consume on-chip resources. The addition of monitoring signals creates an increased burden for the router which must now incorporate the additional signals while continuing to meet timing constraints. These tools primarily rely on the limited on-chip memory for data storage and may compete with the user's design for these resources. Graphical user interfaces restrict interaction with the design since they can not be scripted as part of an automated testbench or integrated with other tools. Unlike software debuggers or test environments, these tools have no programmatic or script-engine capability of accessing or manipulating the design despite interest in such features [23].

These techniques suffer from long turnaround times since they are integrated into the design. The entirety of the design must be run through the tool flow, regardless of which portion or even how little of it has changed. In a worst-case and all too frequent scenario, a developer may only be able to test one revision per day, whereas in software development it is common practice to unit test or debug code directly on the target machine upwards of tens of times per day. Debugging may require multiple revisions in order to fully understand the design flaw.

To demonstrate the often lengthy time requirements for design turnaround, Table 2.2 presents Xilinx ISE 9.2i place-and-route CPU times for five non-trivial designs performed on a 2.66 GHz Intel Core i7-920 processor, targeting an XC4VFX100 part [20]. Blank table entries represent implementations that did not produce a result after 30 hours and that were terminated. The Xilinx MicroBlaze processor [24] is a configurable, pipelined, soft-core processor, 
Table 2.2: PAR times for non-trivial designs with typical timing goals.

\begin{tabular}{|l|l|l|l|}
\hline Design & $f_{\text {clk }}(\mathrm{MHz})$ & $\begin{array}{l}\text { Flat design } \\
\text { implementation } \\
\text { (minutes) }\end{array}$ & $\begin{array}{l}\text { Normal floorplan } \\
\text { implementation } \\
\text { (minutes) }\end{array}$ \\
\hline 5 Microblazes & 127.4 & 330 & 80 \\
\hline 10 Microblazes & 127.4 & - & 270 \\
\hline 20 Microblazes & 125 & - & - \\
\hline 3 CFFTs & 256.4 & - & 35 \\
\hline 6 CFFTs & 250 & 75 & - \\
\hline
\end{tabular}

whereas Complex Fast Fourier Transforms (CFFT) are commonly found in DSP applications. These results make evident that even a few design iterations might result in week's worth of work, provided that the designs achieve closure at all. The time required for placeand-route is a common complaint amongst designers in surveys [25] and is responsible for lengthy verification cycles.

\subsubsection{Assertion-Based Verification}

Assertion-Based Verification (ABV) or simply assertions provide designers a means of verifying simulation designs by specifying conditions that must or must not occur. This requires an extensive understanding of the design in order to formally capture and characterize the assertions and how they must be formulated. There are two classes of assertions: static assertions are mathematically proven against the design, while dynamic assertions are performed during an extensive and exhaustive simulation. Neither method can provide complete coverage of all possible execution branches, but are widely accepted as necessary for verifying designs prior to production.

Assertions can be specified through a wide variety of different languages and environments. Verilog and VHDL both have language facilities to implement assertions and are defined 
in IEEE Standard 1364 [26] for Verilog and IEEE Standard 1076 [27] for VHDL, as does SystemC [28] and System Verilog [29]. Cadence's e [30,31] (formerly known as SpecMan) and Bluespec [32] are two examples of commercially supported languages that can be used for assertion-based verification.

\subsection{High-Level Language Synthesis}

The efficiencies of high-level programming languages are an attractive goal for hardware design. High-level language synthesis (HLS) aims to provide the same level of abstraction to hardware that is provided to software, isolating the source code from the underlying architecture which allows the program to be built for different platforms. HLLs allow the design to be layered, separating algorithms from low-level implementation details, thereby simplifying maintenance. HLS on the other hand, is still in its infancy and is not yet the equivalent of a HLL for hardware development.

Designs are typically first prototyped in an HLL, such as $\mathrm{C}, \mathrm{C}++$, or Matlab, where functional and algorithmic aspects of the design are specified using complex control constructs [33]. However, HLLs are untimed and do not capture the necessary architectural details for the complete specification of a hardware implementation. High-level models serve a critical purpose in providing a reference or "golden" design for development and are referenced throughout the development cycle to determine functional correctness. These comparisons are often manually performed and limited by the restricted access to the physical design.

High-level programming languages were developed to provide a level of abstraction above the underlying hardware architectures. As software design methods evolved - from the earliest programs being coded directly in machine code, then later in assembly languages - the need 
for platform independence quickly became evident. The advent of compilers and high-level languages makes this abstraction possible, changing the programmer's view of hardware from physical registers and architecture-specific opcodes to meaningful, symbolic variables, complex control constructs, data types, and universal operators. Pursuit of a similar paradigm for hardware design was inevitable.

HDLs, such as Verilog and VHDL, provide a similar, but lower form of abstraction than those of software languages. HDLs are Register Transfer Level (RTL) languages, describing the flow of signals between registers and the operations on those signals. RTLs can further be divided into two variants: behavioral and structural RTL. Behavioral RTL describes a design by its behavior in terms of high-level operations such as if-else or other control statements and operations such as addition or subtraction.

Structural RTL provides the lowest-level of specification, instead declaring the structures of the circuit and the connections between them. Structures can be primitive operations such as Boolean or bitwise operations or even the macros provided by the architecture. Structural RTL is also used to design high-performance cores since more control is possible to reduce space requirements or ensure timing will be met. Designers using structural RTL have precise control over each signal, yet the implementation of such a design can be laborious, confusing, and error-prone.

Behavioral representations allow the design to be described using more abstract, higherlevel expressions, such as defining an addition operation to occur on two signals rather than having to explicitly specify the implementation of an addition across the entire signal. Most notably, behavioral languages can specify events for signal transitions, such as clock edges, and have control constructs similar to those in high-level programming languages. These two features alone can be used to describe complex and fundamental design elements, such as state machines. Behavioral language constructs are further subdivided into synthesizable and 
non-synthesizable, where the latter provides even more complexity and abstraction suitable for design testing.

HLS is an extension of these abstractions for FPGAs where a subset of a high-level language is processed to produce synthesizable RTL. HLS requires that the design entry be critically analyzed for structure and flow in order for the translators to correctly interpret the intent. Important constraints in hardware design, such as size and power, are no longer within the precise control of the designer. The abstraction performs its intended function, in part. Designs entered using HLS resolve to functional correctness far quicker than when using HDLs. Yet, long verification cycles still remain. Designs must be precise in their implementation because integration with traditional verification tools, such as formal equivalency, can be difficult or even impossible. Despite the front-end language portability of HLS, designs tend to evolve around the specific compiler being used, defeating the objective of portability. HLS designs also perform poorly in coverage tests because of the additional overhead that these tools introduce. The translation of HLL to RTL may produce artifacts, such as unreachable states or logic that can never be activated, known as dead logic. Debugging at the RTL level or integrating third-party IP are hampered by the unintelligible machine-generated RTL. Additionally, the untimed nature and various transformations from HLL to RTL make cosimulation and debug difficult, if not impossible. Often, the only solution is to attempt to debug the generated RTL which is frequently unresolvable to the original design [34]. An example design of a counter implemented in Accelerated Technologies ImpulseC is shown in Listing 2.2, while an abbreviated synthesizable netlist generated from the code is shown in Listing 2.3.

The listing shows a few of the disadvantages of using HLS. While a counter is a trivial function, the HLS implementation is not. The required overhead code is compiler-specific, requiring numerous special calls in order to set up and handle input and output. These 


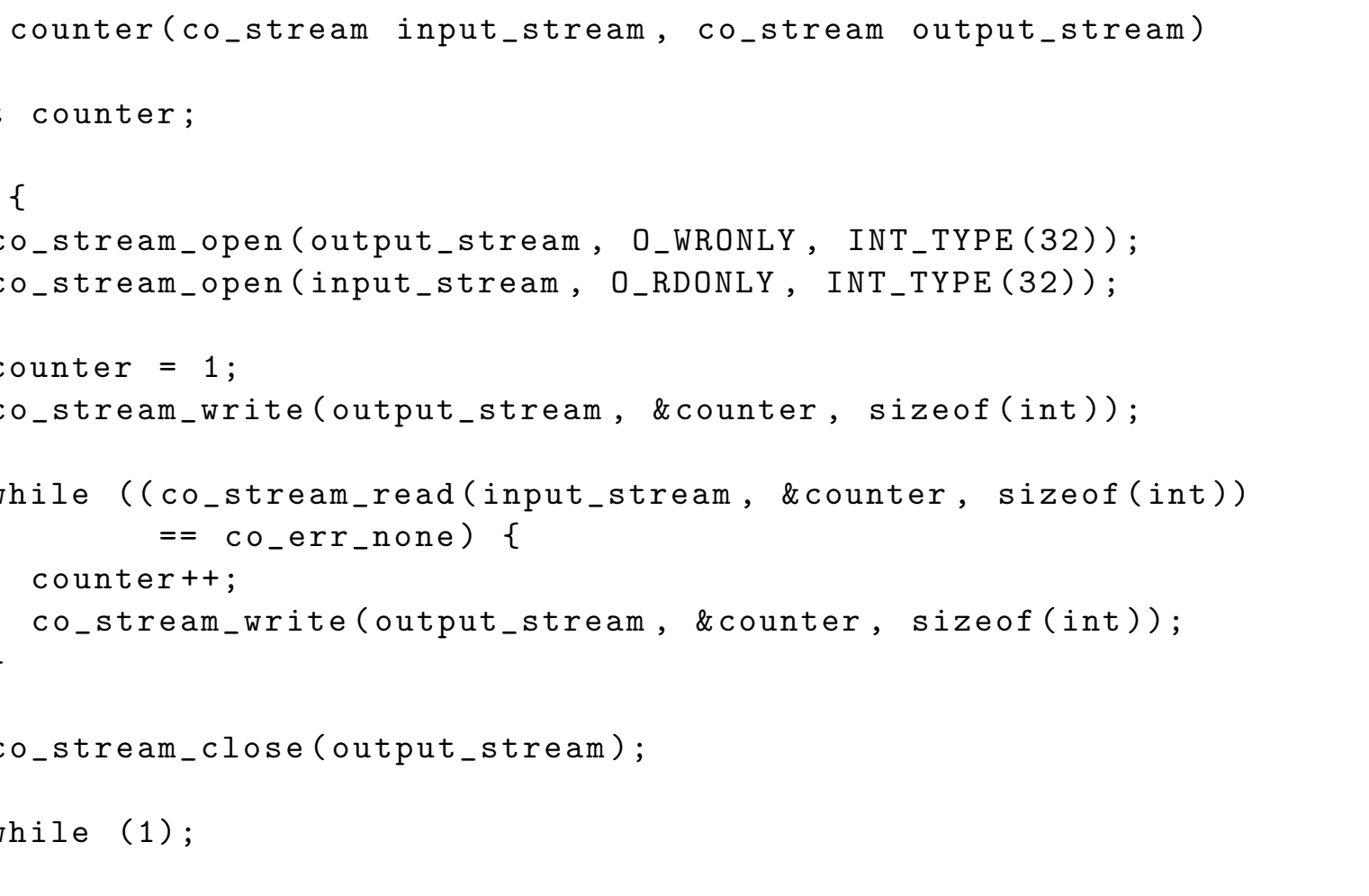

Listing 2.2: Implementation of a counter with HLS.

sections are not portable, as is a high-level language. The example uses blocking First In, First Out (FIFO) queues to control execution without the need for flags or signals. A read or write request will block until the FIFO is ready. The use of FIFOs in this manner simplifies a design, making it a streaming operation without additional overhead or checks.

The resulting synthesizable HDL exhibits more complexity than a hand-written counter. Nowhere in the HDL can the actual counter - a simple addition-be discerned. In a more complex module, it would be even more difficult to try and locate signals of interest to debug or integrate additional IP. 


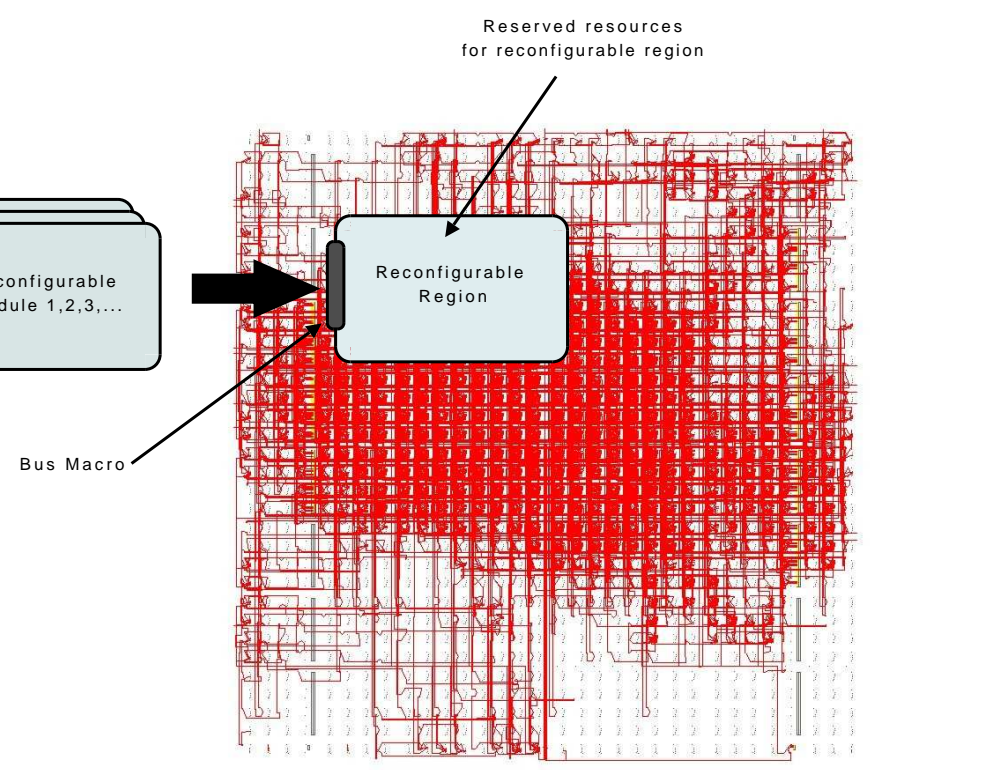

Figure 2.8: Partial reconfiguration for multiple logic modules in the region.

macro creation and placement was a manual task. Automated bus macro placement was the subject of a recent research topic at Virginia Tech [37] as part of the PATIS project, and was eventually eliminated from the vendor's build process altogether in favor of another method. The interface between the reconfigurable region and the rest of the design is critical to reliable, error-free operation. Alignment between the two is analogous to constructing two physical, interlocking parts. During reconfiguration, I/O between the reconfigurable region and the rest of the design must be suspended. Furthermore, the I/O ports must be properly physically aligned on all reconfigurable modules. Reconfigurable regions are restricted to only rectangular shapes (as opposed to ASICs where rectangular shapes are merely a convention) and developers are responsible for placing and sizing the region within the FPGA so that it has sufficient resources for all of the candidate modules. If a module with different resources or interface is later added to the set of reconfigurable modules, this may disturb the floorplan of the static region. Resource estimation floorplanning was the subject of another area of research in the PATIS project [38]. 
Besides the increased burden of development, designers are reluctant to adopt RTR because of its dynamic nature. In RTR, the act of swapping modules into and out of the reconfigurable region turns a single design into multiple, distinct designs, increasing the test space. Unlike most components of a manufacturer's portfolio, RTR cannot be simulated and developers must develop directly on the hardware where visibility is limited. This uncertainty and the resulting difficulty in verifying such a design are a few reasons that commercial designers avoid RTR.

\subsection{Summary}

FPGAs contain not only programmable logic, but many are available with an assortment of specialized processing cores such as microprocessors, memories, DSP cores, and specialized I/O cores. Combined with the capability to realize and implement an arbitrary function while also extracting parallelism, this flexible architecture provides a performancecompetitive platform on which to produce high-speed, space-efficient, low-power streaming applications. FPGAs are capable of processing high-speed streams of continuous data, rather than the non-deterministic read-buffer-process-write cycle required with conventional computer architectures.

The great flexibility and power of FPGAs comes at a price: the design process is complex, time-consuming, and prone to subtle problems. Unlike software which has a widely accepted and open process with a wealth of independently contributed tools, FPGA tools are proprietary and vendor-specific. The complexity of FPGAs is instrumental in the difficulties encountered when trying to debug and diagnose problems. The lengthy implementation cycle, as well as the lack of visibility into a design are the most significant obstacles. Alternative design methods, such as high-level languages which were elemental to the rise of 
computers and software, has not made FPGAs more accessible in the same manner. Rather, HLS faces other obstacles to acceptance.

A unique capability of FPGAs is the ability to dynamically reconfigure a select region of the device. This capability was previously commercially inaccessible or undesirable because of its complexity and uncertainty in production environments, however it holds significant promise in development and debug environments. A review of debug-related research and products reveals partial reconfiguration as an unexplored and potentially useful tool in accelerating the debug process. 


\section{Chapter 3}

\section{Related Work}

The FPGA debugging field has amassed a large body work. The goal of developing an intuitive and productive means of debugging is ambitious given the closed nature of FPGAs and their prominent role in electronic system design. The earliest implementations have their roots in the ASIC market, many based around long-standing industry standards such as JTAG which were developed to verify individual components as well as entire systems. FPGA-specific products are firmly rooted in leveraging on-chip components, such as JTAG cores or embedding specialized debug cores into the design.

\subsection{Categorization of Debug Approaches}

A review of commercial and research FPGA debugging products reveals two prominent approaches: embedded logic analyzers and JTAG-based analysis tools. Embedded logic analyzers recreate the familiar interface of physical logic analyzers but are implemented as part of the FPGA design by inserting special cores wired directly to signals of interest. These cores rely on a capture methodology where the selected signal's activity is internally recorded 
beginning when predetermined conditions are met. While some limited changes may be made at runtime, the trigger conditions that initiate the capture in addition to the selected wires are implemented as part of the design's logic and specified at design time. As part of the physical design, these cores compete for system resources, specifically on-chip RAM. Captured signal traces are stored on-chip until read out via JTAG and displayed in a graphical user interface resembling a simulator's waveform viewer. Interaction with the design is limited: neither debug scenarios nor tasks can be scripted into a larger comprehensive or automated test framework. Capture conditions cannot be appreciably modified without re-implementing the entire design, and different parts of the design cannot be inspected other than those whose signals were initially specified. However, designs can typically run at or close to their target operating frequency.

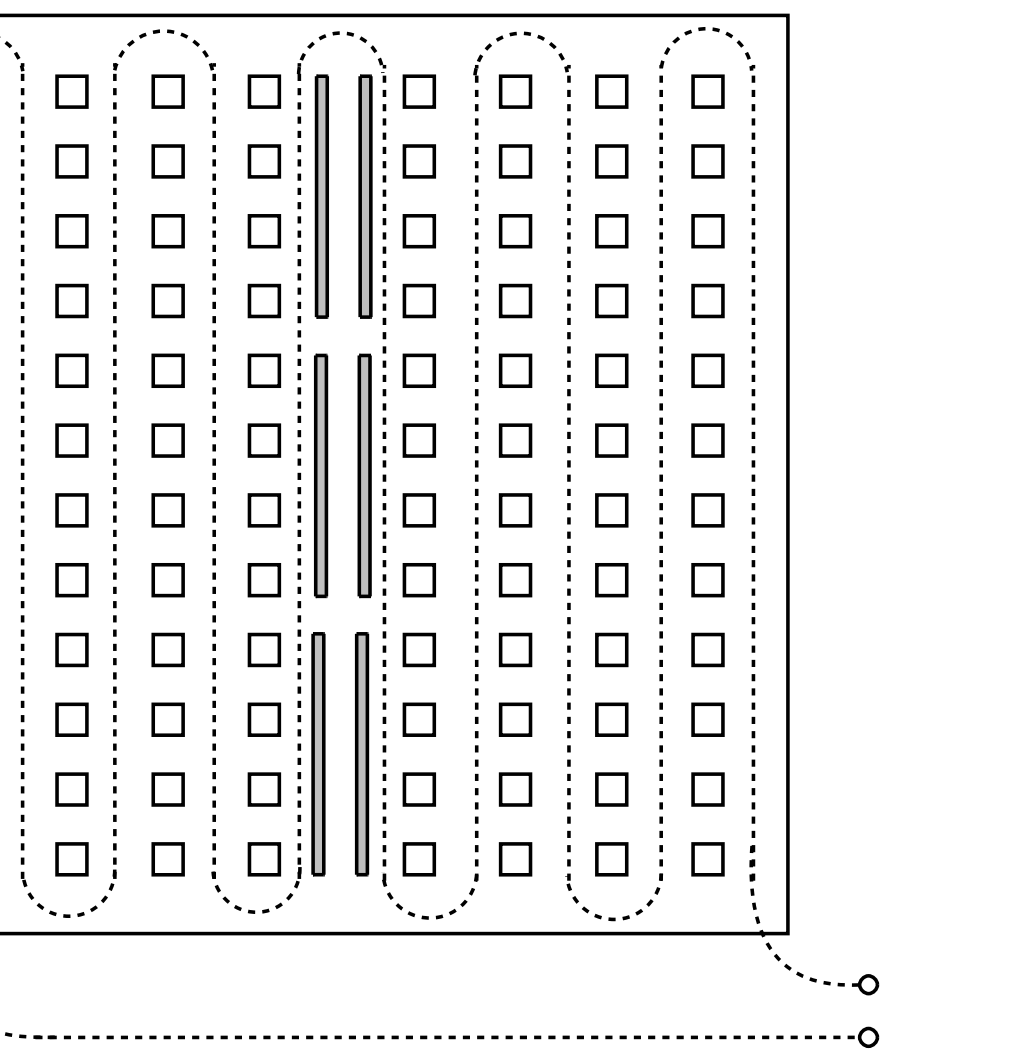

Figure 3.1: JTAG chains visit every resource in the FPGA. 
Table 3.1: Timeline of Xilinx Boundary Scan chain length.

\begin{tabular}{|l|l|l|}
\hline $\begin{array}{l}\text { Release } \\
\text { Year }\end{array}$ & Device & $\begin{array}{l}\text { Approximate Size of } \\
\text { Configuration Chain (bits) }\end{array}$ \\
\hline 2002 & Virtex-II Pro XC2VP100 & $34,272,000$ \\
\hline 2004 & Virtex-4 XC4VLX200 & $51,367,424$ \\
\hline 2006 & Virtex-5 XC5VLX330T & $82,696,192$ \\
\hline 2009 & Virtex-6 XC6VLX760 & $184,823,072$ \\
\hline 2011 & 7 Series XC7V2000T & $447,337,216$ \\
\hline
\end{tabular}

The other prominent debug approach utilizes JTAG for state inspection. JTAG is an acronym for the Joint Test Action Group, an industry board assembled to define the standard now found in IEEE Standard 1149.1 [39]. JTAG is a generic, non-proprietary means of debugging and controlling electronic components, embedded in a range of products from simple components to sophisticated processors. While JTAG tools do not require the invasive instantiation of cores and routing of signals as found in embedded logic analyzers, it is slower to operate. A snapshot of the device state is captured internally and must be serially shifted out in its entirety for inspection. Figure 3.1 shows a conceptualized JTAG chain winding throughout the entire FPGA, passing by each resource. The snapshot typically requires that the design's execution be halted. Once retrieved, any state register of the design can be inspected.

JTAG-based tools offer complete visibility without significantly altering the design as is the case with embedded logic analyzers, however they are limited in how much control can be exerted over the design. JTAG-based tools do not offer the ability to control an FPGA design's execution, nor can the debug scenario be altered. JTAG operates at a significantly slower clock rate than the average FPGA design and as devices increase in size, density, and complexity, the time required to shift the entire chain out proportionally increases. A simplified schematic representation of a JTAG chain is shown in Figure 3.2 and Table 3.1 shows the size of the configuration bitstream for the largest of the major device releases since 
2002 [40-44]. The configuration bitstream is a good approximation of the boundary-scan chain length.

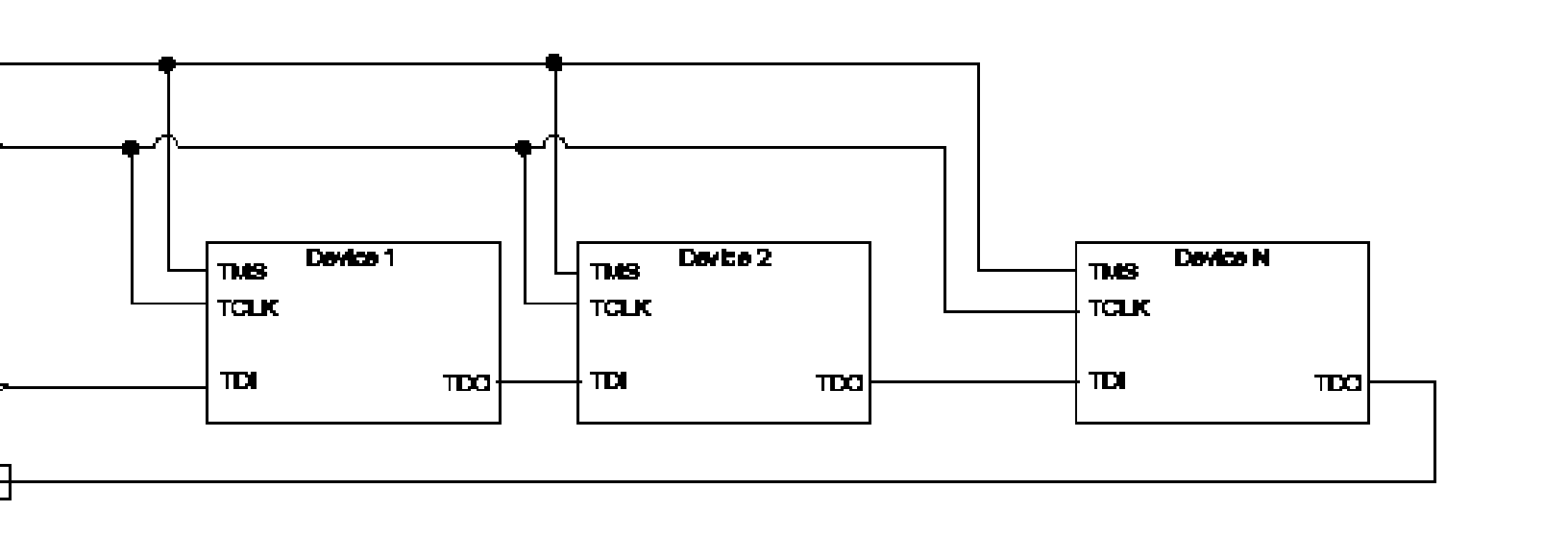

Figure 3.2: JTAG daisy chains require few connections.

JTAG requires few connections but is flexible enough to allow devices to be daisy-chained. Each JTAG-enabled device is manufactured with vendor- and model-specific identifiers so that multiple devices can be placed into the same chain and still be uniquely identified. JTAG requires only small number of input and output pins making it easy to place on the smallest of component boards. As such, it has become the defacto standard for debug and testing on virtually all electronic devices. It is found on virtually all FPGA devices.

As shown in the Figure 3.2, the header's external input is wired to the first device's TDI pin; in turn, its TDO output is connected to the next device's TDI input and so on until the final device's TDO pin is wired back to the header. A single master clock and device select are globally wired to all devices of the chain. One of JTAG's weaknesses stems from its simplicity. The sole data line restricts data transfer to a serial model throughout the entire chain. JTAG's modest clock rate makes this model unsustainable as devices and likewise chain length increases. Rapidly increasing FPGA densities make JTAG less practical for future devices. 


\subsection{Commercial Debug Products}

\subsubsection{Vendor Products}

The proprietary nature of FPGAs restricts development of debug products mostly to the original manufacturer. However there have been a few notable third-party contributions. By far, vendors prefer developing embedded logic analyzers (ELA), extending the waveform viewer window found on simulators into the debug environment. Xilinx's ChipScope Pro [45] and Altera's SignalTap II [46] are two examples of the embedded logic analyzer class of tools. ELAs require that specialized cores be instantiated as part of the design. Signals of interest are manually selected through a user interface. The entire design is then run through the standard tool flow. Figure 3.3 shows how different components of Xilinx's ChipScope Pro interact with and become deeply integrated into the design. Wires for the framework and signals of interest are routed globally while the debug cores compete with the primary design for resources. The most obvious and limited resource is RAM, which are plentifully required by debug cores since signal traces are stored on-chip until read out and displayed. Modifications to the debug configuration, such as changing which signals are to observed can be disruptive, create routing contention, which in turn may cause the design to fail subsequent timing analyses. ELA tools are often restricted to the top-level design signals and are not able to inspect arbitrary internal signals without the need to modify intermediate interfaces.

\subsubsection{Third-Party Products}

Despite the closed nature of FPGAs, there are several notable commercial products. GateRocket's Device Native Verification [47] system allowed designs to be run directly on an 


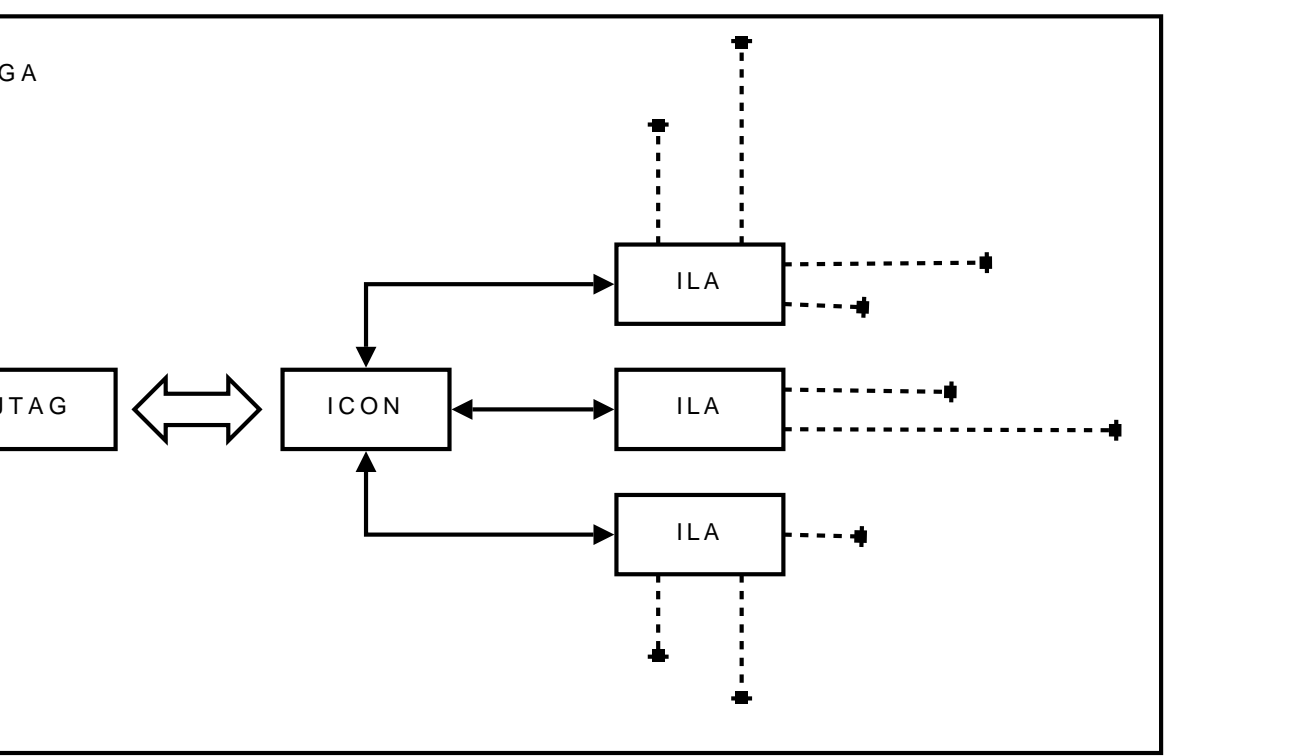

Figure 3.3: ChipScope gathers data through physically routed nets.

FPGA. The system comprised of an external Rocket Drive housing an FPGA which connected directly to a workstation. The system tightly integrated with several simulators, utilizing their user interface and waveform viewers. Individual waveforms could be displayed in the waveform viewer directly from the hardware while bypassing traditional and slower software simulation. Technical details were sparse in public literature and documentation was restricted to customers so the system's architecture was not widely understood. Despite widespread marketing efforts and enthusiastic praise as an innovative approach, GateRocket ceased operations in July of 2011 due to lackluster sales [48].

Sandbyte's FPGAXpose [49] framework provides visibility into all design storage elements by reading their state through a JTAG connection. While eliminating the need to instantiate cores into the design, this exposes the application to the disadvantages of JTAG. JTAG is a comparatively slower interface more suitable for interactive work, rather than full-speed analysis. Like other third-party products reviewed, details of this architecture are not widely publicized. 
Synopsys' Identifty RTL debugger [50] bears a close resemblance to conventional software integrated development environments. Debugging is performed in a source code viewer on the original RTL, or through a waveform viewer. Like embedded logic analyzers, a specialized Instrumentor core is instantiated into the design but rather than recording signal traces, annotations are displayed on the original source RTL. Identify combines traditional integrated software development environment features such as syntax highlighting and context pop-ups for symbolic register values with hardware debugging. The incremental development flow is leveraged which attempts to preserve previous implementations, thereby reducing build times. JTAG is used to shift the FPGA's state out to the Identify user application for inspection.

The Cadence Palladium toolset, part of the Incisive XE suite, is a stand-alone emulator/ accelerator allowing rapid hardware/software co-verification. Product literature claims from 100 up to 1,000,000 times speedup over conventional software-based RTL simulation. Debugging capabilities include embedded logic analyzer access with immediate test point modification without the need for re-implementation, global visibility without the use of physical or routed wire probes, and limitless trace capture [51]. It is unclear what architecture supports these claims.

\subsection{Debug-Related Research}

The most ambitious debug products have been the product of research projects. Researchrelated debug products are often very innovative, but tend to be restricted to specific architectures. Unfortunately, the most exciting debug products are no longer supported.

During development, the NPU processor [52] incorporated custom logic that automatically halted execution and allowed automated, full inspection of the state. NPU is a 32-bit, 
binary compatible processor based on the Intel 486 architecture. Developed on an FPGA, the developers were motivated by some common FPGA obstacles to productivity. Long turnaround times and limited visibility into the design were responsible for slow progress in development. Incorporating custom breakpoint logic activated by the program counter, the breakpoint logic automatically suspended execution and triggered an automated state dump over JTAG. The technique relies on JTAG's ability to push custom instructions into the design and later shift out the device state. The breakpoint address was set by JTAG, and when activated triggered a JTAG shift making the device's internal state available. This research presented several novel approaches to debugging including generously segmenting the design into 11 distinct and manageable JTAG chains to accelerate the otherwise lengthy shift operation, and the automated approach to shifting out state and automatically resuming execution. This domain-specific technique is not suitable for arbitrary designs and aspects of it required re-implementation for modifications to the debug framework.

Researchers at the Lappeenranta University of Technology developing an embedded active magnetic-bearing controller on a Virtex-II Pro FPGA used the device's PowerPC processors to read internal signal data and to debug the design. The authors make note of the difficulty in achieving target clock rates and the large amount of memory required for their debug activities [53]. Approaches such as these tend to be domain-specific and require repeated modification and re-implementation of the design.

An in-situ debugging system developed at the Los Alamos National Laboratory employed JTAG for state readback [54] in a unique way. Based on a Xilinx reference design [55], the researchers extended the capabilities of an external Microchip PIC processor to provide access to both transactions and internal state. The system was primarily developed to aid in debugging otherwise inaccessible installations, such as those deployed into orbiting satellites. The microcontroller directly drives a JTAG chain, through the second of the FPGA's two 
Boundary Scan (BSCAN) modules, leaving the first available for vendor tools. The debug core included a programmable triggering mechanism which enabled system traces to be recorded directly into internal Block RAMs (BRAM). The framework allowed both reading and writing to arbitrary design registers and was accessible from a Linux command-line application.

JHDL [56] developed at Brigham Young University, was first conceived as a high-level hardware description language for RTR applications. JHDL evolved into one of the earliest full-blown design environment supporting high-level design entry, floorplanning, netlist generation, schematic generation, and an integrated debug and simulation environment. Despite the use of Java, JHDL is a structural design environment, enabling rapid design entry using a popular high-level language that appears to have effectively separated the design from the platform, a limitation that plagues conventional HDLs. The target device is specified as part of the design and is easily re-targeted to another supported platform. Low-level, device-specific instantiations are avoided through the use of abstracted component libraries. JHDL also provided several unique perspectives to hardware development. First, both hardware and software could be co-developed in the same environment using the same language. While hardware development occurred by extending preexisting JHDL libraries, software development occurred in the conventional sense. Another unique aspect of JHDL was the combined hardware and simulation environment that could be executed in parallel, effortlessly switching between the two while annotating different views of the design, such as a schematic or waveform view. Development appears to have halted years ago, with the last supported devices being the Xilinx XC4000, Virtex, and Virtex II [57].

Like JHDL, JBits [58] was also Java-based circuit design language. Developed by Xilinx and first targeted towards the Virtex line of FPGAs, JBits provided a hierarchical, object-oriented approach to design entry. Using Xilinx's Runtime Parameterizable (RTP) Core library, a 
parameterizable library interface to Xilinx's core device components, runtime reconfiguration was accessible from a high-level language as were floorplanning capabilities using JPlace. These unique high-level capabilities have not reappeared since JBits was discontinued. JBits was unique in that it allowed direct manipulation of the low-level bitstream implementation from a high-level language. However this direct access made JBits complex requiring the tool to contain architecture-specific details for each of the supported devices. JBits' bitstream manipulation capabilities made it an ideal candidate for hardware-based debugging tools. A project at Brigham Young University used JBits to instrument a JHDL design for debug by directly manipulating the final bitstream and was demonstrated to be effective [59].

The BoardScope [60] debugging suite provided graphical and command-line user interfaces to the design's data flow. BoardScope was integrated with the JBits [59] suite and the DDTScript language which enabled instantiation of a run-time core abstraction that could be precisely placed and manipulated on the FPGA fabric. Inputs to cores could be altered, state read, and bitstreams exported for later use, allowing for interactive debugging [61]. BoardScope never appears to have been offered as a commercial product and is not available for architectures beyond the Virtex-II Pro.

\subsection{High-Level Synthesis}

HLS is in of itself not a debug methodology, but a means of raising the abstraction layer away from the low-level hardware implementation, thereby eliminating the need to debug at that level. HLS offers a direct path to creating synthesizable HDL from an HLL. While HLLs are the fastest, most precise, and abstract means of specifying and validating algorithmic correctness, HLS has yet to gain widespread acceptance for reasons such as poor coverage, large overhead, and dead logic. The automatically machine-generated RTL is difficult to integrate with third-party IP cores and to process with formal verification tools [34]. 
Accelerated Technologies ImpulseC [62] high-level synthesis framework is based off a subset of the C programming language. The compiler is based off the StreamsC compiler [63] developed at Los Alamos National Lab, which in turn relies on the Stanford University Intermediate Format (SUIF) [64] to generate synthesizable netlists from high-level language. The user interface is similar to software development environments, with features such as code completion and syntax highlighting. Like many HLS environments, the machine generated RTL is difficult to reconcile to the original source code, making it difficult if not impossible to manually debug or verify. A complete reference on programming for FPGAs with ImpulseC can be found in [65].

ImpulseC was the basis of at least two notable research projects investigating improving developer productivity. Research done at the University of Florida [66] developed a means of embedding assertions authored from the high-level programming environment into the synthesized netlist, providing more visibility of the synthesized design. The synchronous assertions provided immediate feedback to the developer, increasing confidence and verifiability. An overview of assertion-based verification can be found in Section 2.3.4.

A Virginia Tech doctoral dissertation [36] focused on abstracting away low-level implementation details for partial Runtime Reconfiguration (RTR) into ImpulseC's high-level language. This work was significant in several regards. First, RTR is a low-level, device-dependent technique which requires significant knowledge of both the device and the design. RTR is an unlikely candidate to be transparently integrated into a high-level language. Second, RTR enables a smaller, less-expensive, more power-efficient device to have a region designated for temporally allocating modules for on-demand execution rather than statically allocating all the modules at design time. This satisfies one of the criticisms for HLS that machine-generated RTL designs are less power-efficient than custom RTL designs. 


\subsection{Deficiencies in Existing Approaches}

The previous overview of commercial and academic debug environments betrays a pattern: vendors provide embedded logic analyzers while third-party vendors favor JTAG access methods. This is not surprising. Vendors have the distinct advantage of leveraging the knowledge of their own proprietary architectures and in some cases embedding on-chip debug peripherals to assist. Third-party vendors are left with few options other than the industry-standard JTAG to access the internals of the FPGA or to develop their own interfaces. The primary shortcoming of all the approaches is the lack of visibility into the FPGA.

Third-party providers depend largely on JTAG since it is one of the only standardized interfaces found on all platforms. There is the option using the abundance of I/O that the FPGA has to offer, however this is not as attractive as option as it may seem. The GateRocket and Cadence products sell their own development platforms to leverage this opportunity, providing a higher level of service. There is a drawback to this approach, notably that these platforms are more than likely not at all similar to the final target platform. They most certainly will lack the peripherals, connectors, and configuration of the final platform. Porting to another device, even to a different sized part within the same family, can be problematic particularly with regards to placing and routing the design.

The benefits of the vendor's embedded logic analyzers are clear, however there are drawbacks to the approach. These tools are sealed within their user interface which makes integration with other tools difficult. The primary disadvantage of ELA-style approaches is the semipermanence of the chosen context. That is, the use of these tools is akin to a design decision. The debug facilities become deeply integrated into the design, particularly in regard to wire routing. Memory requirements are also a difficult trade-off. Onboard memory is hardly ever sufficient for the design and with ELA it must be shared. Memory use must be delicately balanced between the number of signals to monitor and the number of samples to record. 
Table 3.2: Summary of debug products.

\begin{tabular}{|c|c|c|c|c|}
\hline Source & Product & Class & Comments & Status \\
\hline Vendor & $\begin{array}{l}\text { Xilinx } \\
\text { ChipScope Pro }\end{array}$ & ELA & $\begin{array}{l}\text { Instantiated cores, on-chip } \\
\text { trace recording, graphical user } \\
\text { interface }\end{array}$ & Available \\
\hline \multirow{4}{*}{ Third-Party } & GateRocket & Proprietary & $\begin{array}{l}\text { External platform; waveform } \\
\text { viewer integration }\end{array}$ & $\begin{array}{l}\text { Defunct } \\
\text { July } 2011\end{array}$ \\
\hline & $\begin{array}{l}\text { Sandbyte } \\
\text { FPGAXpose }\end{array}$ & JTAG & $\begin{array}{l}\text { No embedded cores, complete } \\
\text { state visibility }\end{array}$ & Available \\
\hline & $\begin{array}{l}\text { Synopsys } \\
\text { Identify RTL }\end{array}$ & JTAG & $\begin{array}{l}\text { Embedded core; annotations } \\
\text { on waveform viewer, source } \\
\text { code viewer }\end{array}$ & Available \\
\hline & $\begin{array}{l}\text { Cadence } \\
\text { Palladium }\end{array}$ & Proprietary & $\begin{array}{l}\text { External, stand-alone acceler- } \\
\text { ator; software co-verification; } \\
\text { embedded logic analyzer; } \\
\text { rapid test point modification; } \\
\text { very expensive; targeted } \\
\text { toward ASIC development }\end{array}$ & Available \\
\hline \multirow{3}{*}{ Research } & JHDL & Unknown & $\begin{array}{l}\text { High-level language with inte- } \\
\text { grated debugging and simula- } \\
\text { tion support; rapid perspec- } \\
\text { tive change between simula- } \\
\text { tion and on-hardware debug- } \\
\text { ging }\end{array}$ & $\begin{array}{l}\text { No longer } \\
\text { supported }\end{array}$ \\
\hline & JBits & Unknown & $\begin{array}{l}\text { Direct manipulation of bit- } \\
\text { stream; rapid debugging; lim- } \\
\text { ited to select devices }\end{array}$ & $\begin{array}{l}\text { No longer } \\
\text { supported }\end{array}$ \\
\hline & BoardScope & Unknown & $\begin{array}{l}\text { Graphical and command-line } \\
\text { support }\end{array}$ & $\begin{array}{l}\text { No longer } \\
\text { supported }\end{array}$ \\
\hline
\end{tabular}

Capture methodologies are not always appropriate for low-level debugging. In addition to Xilinx's ChipScope Pro, the offerings from Sandbyte and Synopsys fall into this category as well.

The obstacles to providing a fast and visible architecture for debugging are the limited access points into an FPGA and an unobstructed view of the design. JTAG has been a reliable and robust standard for debugging for well over a decade. However it is slow, serial, and typically applied globally across the device. With JTAG, the debug vantage point is the 
equivalent to that of a long, narrow tube: significant time and effort is required to pass information along the tube and the tube must be emptied each time, regardless of how much information is relevant. A summary of the debug products covered in this section is given in Table 3.2. Domain-specific approaches, such as those implemented for the NPU processor, the Lappeenranta University project, and the work undertaken at Los Alamos were not included. The most innovative approaches, those generated from research projects quickly faded. There is little documented evidence to explain why these projects failed to achieve commercial success. However the growing concern over IP protection suggests that tools such as JBits, BoardScope, and JHDL exposed too much of the internal architectural details.

\subsection{Summary}

Debug solutions are limited. Vendors provide embedded-logic analyzers to extend the original development environment found in simulation to debugging, while third-party vendors are rather limited in their approaches. Applications for JTAG are the most common, however the least likely to scale to future platforms. JTAG is a universally adopted and open standard, but places the debugging context at the end of a slow serial line. Some third-party approaches also include stand-alone development platforms alleviating some of the shortcomings, however this does not permit development directly on the final target platform. The most innovative approaches have been research products, but these typically exposed more architecture-specific details.

A significant shortcoming in almost all the approaches reviewed is one of perspective. The debugging context is placed outside the FPGA, with a restricted view onto the FPGA's state. Embedded solutions, such as those requiring cores to be instantiated into the design 
are based on capture methodologies. The storage requirements limit the width or depth of information viewable. With JTAG solutions, this view is serialized requiring the entire state to be shifted out regardless of how much information is needed. A new perspective, one from within the FPGA with random access, is needed. 


\section{Chapter 4}

\section{Improving Abstraction and Turnaround Time}

The model verification gap and the deficiencies discussed in the previous chapters highlight some of the weaknesses in FPGA development flows. The model verification gap exists because high-level reference models are used primarily to verify against simulation models and because they can not be directly coupled to the hardware. In many cases, validation against high-level models is manual. Furthermore, there are significant gaps in the ability to analyze the implemented hardware in detail without compromising time or accuracy.

FPGA development environments lack the flexibility found in software development environments, most notably intuitive means for quickly testing modifications and validating designs. Three prominent attributes of software development are identified and targeted in this research for FPGAs: visibility, controllability, and agility.

Visibility is the extent to which design elements, such as signals, ports, and registers, are observable once implemented in hardware. In software debugging, special annotations such as symbol tables are compiled into the binaries and performance-enhancing optimizations 
are avoided to improve the observability of the application. Once a variable is in scope, it can be printed or "watched" within a debugger where changes to the variable are trapped. No such standardized facility is natively available in FPGAs other than diagnostic LEDs, and debugging remains largely technology- and vendor-dependent as well as a learned skill. Controllability is the extent to which a design's state can be manipulated or altered during execution. Examples include forcing variables to values at runtime, a feature widely supported by software debuggers but not architecturally supported in FPGAs. Altering the state of the debug mechanism is also not natively supported. Software breakpoint modifications do not require re-compilation or restarting of the affected unit, while most FPGA vendor tools implement capture or trigger mechanisms as part of the monolithic design flow. Finally, agility is defined as the ease and efficiency at which modifications can be made to a design. It is a common occurrence during FPGA development that a trivial change requires a re-implementation of the entire design, a lengthy process taking potentially tens of hours for large designs [20]. Software build tools such as Make selectively rebuild only the affected units within the dependency tree of the modified unit. FPGA flows have support for incremental or module-based development methods, but these have strict requirements which are difficult to attain. Agility as applied to debugging is similar. While most vendorspecific means of debugging and design analysis are built as part of the design, agility in this context refers to modifying the debug configuration.

There are two phases of the development cycle where these weaknesses are most prominent: initial modeling and hardware validation. High-level reference models made during initial design planning lack integration with the rest of the flow. This inability to integrate reduces the model's effectiveness and hinders productivity during validation. Later on during lowlevel debugging, the lack of insight into executing hardware during runtime and the inability to inspect it are significant shortcomings of all FPGAs. These are addressed through two separate approaches, High-Level Validation and Low-Level Debug. 
The remainder of this chapter is organized as follows:

\section{High-Level Validation}

In Section 4.1, the High-Level Validation (HLV) framework is presented. HLV seeks to couple a normal, high-level reference model written in a standard HLL to synthesized and implemented hardware without significantly modifying either while simultaneously raising the layer of abstraction for validation. In essence, HLV serves as a software-based, unittesting framework for hardware. Aspects of mapping software and hardware elements are discussed.

\section{Low-Level Debug}

In Section 4.2, the low-level aspects of improving debugging time turnaround are discussed. Low-Level Debug (LLD) changes the perspective of FPGA debugging from an external viewpoint to an internal one, removing much of the latency and design-time commitments from debugging. LLD changes the model of analyzing FPGA state from the conventional serial JTAG analysis method to treating the FPGA as a random access memory where state can be arbitrarily analyzed. Additionally, LLD leverages the rapid implementation times of partial reconfiguration by separately partitioning off debug logic so that it can be rapidly swapped out without disrupting the rest of the design. LLD retargets the three attributes of software development - controllability, visibility, and agility - for FPGAs.

\section{Dynamic Modular Design and Validation}

In Section 4.3, the Dynamic Modular Development (DMD) project is discussed. DMD is the overall framework in which this debugging framework is presented. DMD aims to improve turnaround time for the entire range of FPGA development tasks including floorplanning and implementation time as well as the debugging aspects discussed here. PATIS, a major component of DMD, is an automatic floorplanning tool that isolates all top-level modules for efficient design turnaround. PATIS is capable of adjusting floorplan boundaries in two 
dimensions while also balancing resource requirements for each module. PATIS' floorplan database is populated in the background and ensures that a suitable floorplan is always instantly available without stalling the development process as a new one is produced.

\subsection{High-Level Validation}

The HLL functional models that are created during the first phases of the development cycle serve a critical role throughout the process. In addition to providing a guideline from which to build the hardware, they also provide critical reference data by which to validate the design's accuracy. The model verification gap exists because the reference model is physically isolated from the subsequent design steps. HLLs are not natively compatible with HDLs and high-level synthesis has not yet fully matured or been standardized for widespread use. Any validation that occurs between the reference model and subsequent design steps, such as simulation or hardware, are mostly manual or require significant user intervention. Bridging this gap by directly linking the model and simulation is a viable solution, but of limited usefulness. Both the model and the simulator can run on the same platform and frameworks such as the deprecated Program Language Interface (PLI), now succeeded by the Verilog Procedural Interface [26], provide a convenient and structured means of interfacing HDL simulations to C-language programs. However the vagaries of simulation, such as unintended differences between the simulation model and the actual implementation, make this an unreliable strategy. Instead, a means of linking the reference model directly to the hardware would provide a robust means of directly validating hardware without the use of HLS.

At first glance, high-level reference models share little in common with hardware implementations making them unlikely candidates capable of being coupled. HLLs do not characterize 
any timing constructs and are mostly algorithmic in nature. Hardware designs leverage concurrency and require skillful pipelining for the best designs. Procedural languages provide a convenient mechanism by which to model a hardware design's structure. Individual procedures or functions provide a convenient means of logically segmenting a functional model and mapping it to individual hardware modules. Hardware modules and software procedures have much in common and are the most logical pairing for mapping between the two. Both have well-defined boundaries that hide the functionality, state, and implementation from the outside. In software, the term scope defines the context wherein variables exist and to what other areas of the design these are visible. Both also have a well-defined gateway through which information passes, known as an interface. In software, the function's arguments defined by its signature or prototype are the primary means of passing data into and out of the procedure. In hardware, the module's ports define this interface. Modules and procedures are also a convenient means by which to reuse functionality. In both cases, repeated use of functionality warrants that it be encapsulated or refactored into a separate procedure or module so that it can be repeatedly and reliably be instantiated where needed. Figure 4.1 shows how refactoring frequently used code into a function improves the maintainability of the code as well as reduces the likelihood of coding errors. In addition, such refactoring may improve other metrics such as final design size.

Mapping an untimed reference model to hardware can occur only if three conditions are satisfied. First, functional entities in each must be logically mapped to one another. In the simplest case, each procedure could be mapped to a corresponding module. Second, the data inputs and outputs of each should also be mapped. The simplest case again is a direct one-to-one mapping. Control signals, which are discussed in Section 4.1.2, are excluded from this mapping. Finally, the interaction of the control signals and the latency of the hardware module must be modeled in software. All three can be accomplished without invasive modification of either the reference model or the hardware. 

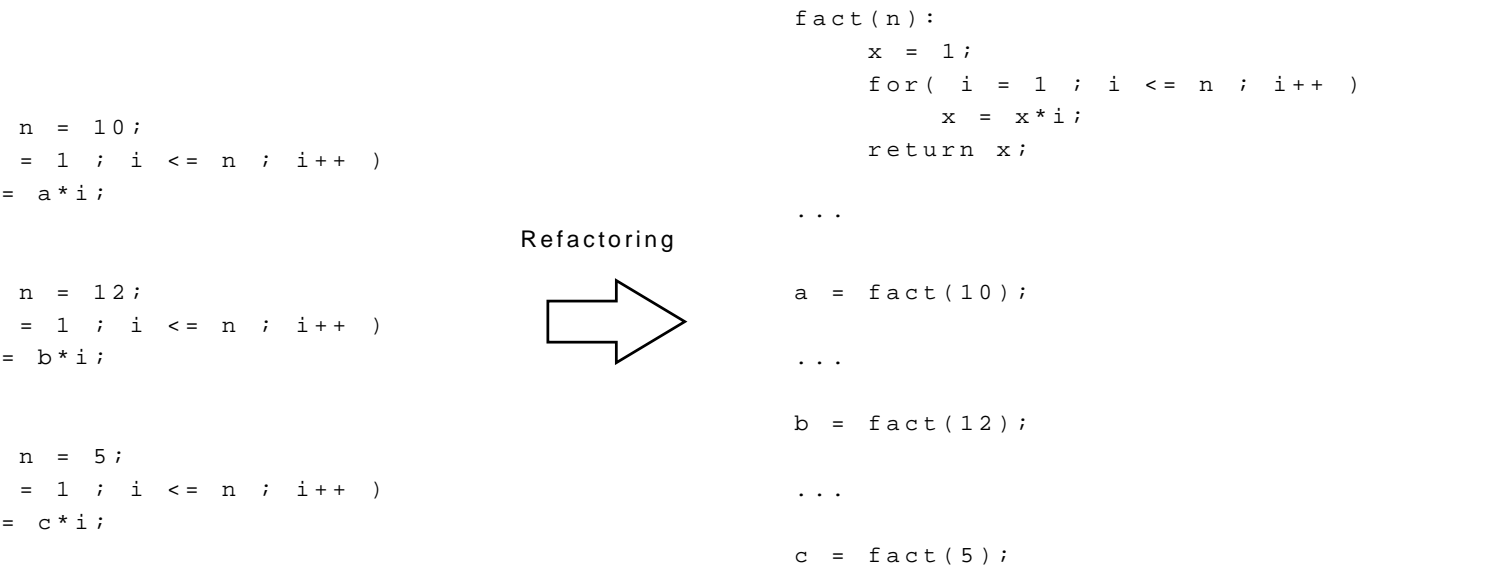

Figure 4.1: Refactoring code improves maintainability.

\subsubsection{Mapping Software Procedures to Hardware Modules}

HLLs are untimed, meaning that the actual execution time, measured either as real ("wallclock") time or as a function of any parameter related to the platform on which it is running, is irrelevant. Hardware design on the other hand is characterized by its execution time as a function of the number of clock cycles required or latency. While the purpose of the reference model is to clearly define the desired and correct results for given inputs through an algorithm, additional metrics apply to hardware. Hardware execution is bounded by the time required for each individual stage of the operation or through some signaling mechanism to indicate that an operation has completed. Despite this mismatch, it is still possible to map a functional software representation to a hardware model.

Procedures have well-defined boundaries and a single point of entry. Likewise, hardware modules also have well-defined boundaries and it is through this similarity that a wellpartitioned software model can be effectively cast in hardware. Figure 4.2 illustrates a one-to-one mapping of hardware and software elements. 

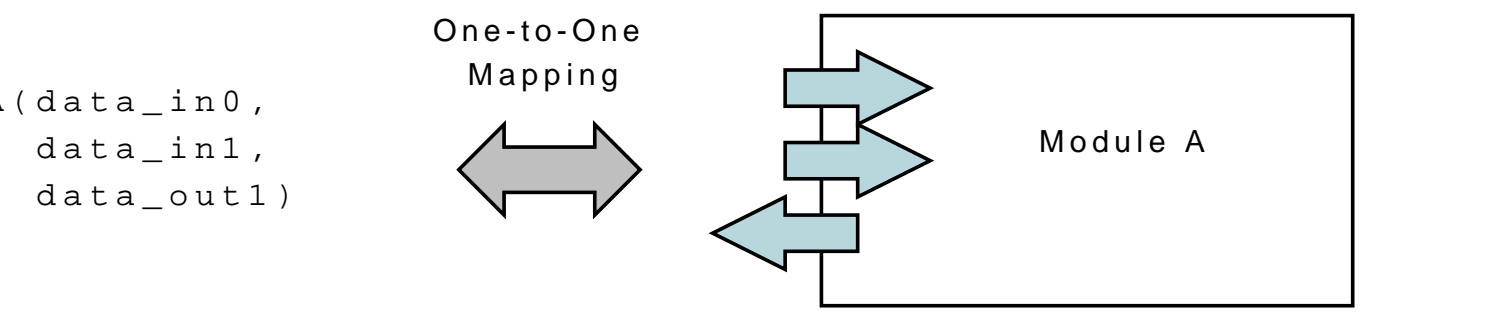

Figure 4.2: Mapping data paths in hardware and software.

\subsubsection{Mapping Data and Control Signals to the Software Model}

Mapping the interface of hardware modules to that of a software procedure is rarely a simple one-to-one mapping. Hardware module interfaces have two types of ports, data and control, while software procedures do not typically address these hardware constructs. Data signals are easily matched to the corresponding data variables of the software model since the model is algorithmic in nature. Control signals are a broad category of signals that can indicate actions to be taken such as an operation; the different states of the circuit such as busy, ready, or acknowledge; or meta-information about data signals. For example, pipelined designs have several stages in which distinct operations are performed. As the data moves through the pipeline, each stage must coordinate with its neighbors. A common scenario in pipelined designs is that valid data may not be available for each clock cycle, thereby introducing stages that are empty or contain invalid data. These empty stages are known as stalls or bubbles. Since hardware execution can not be stopped, the stages of the pipeline must also propagate which data is valid. Invalid data in a pipeline is a normal occurrence and caused when pipelines must wait for data to become available, such as the time in between image frames in video processing applications or the service time required for a memory access. Control signals are shifted along the pipeline with the data to indicate that the data is valid and not the result of a stall. In software models, control signals are not considered since they are not part of the algorithm. The simulation model is the first time in the development cycle that control signals are considered. However, by leveraging the 
low-level detail of signal interaction visible in simulation, a software model of control signal interactions can be extracted. This model can be integrated into the reference model thereby creating a means of interacting directly with the hardware implementation. An overview of this process is illustrated in Figure 4.3.

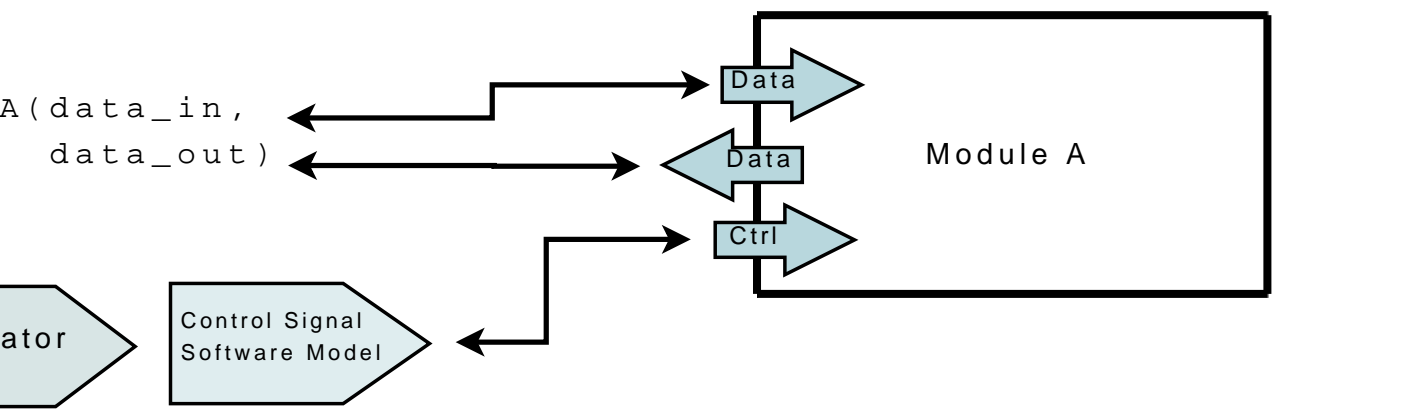

Figure 4.3: Developing a software model for hardware control signals from simulation models.

\subsection{Low-Level Debug}

Lack of low-level visibility, control, and agility of a design is the other deficiency of FPGA development methodologies. Once the design has been implemented, it is difficult if not impossible to see what the precise state of any register is at any given time, arbitrarily stop the design to inspect registers, or quickly change the debugging configuration. In comparison, all these tasks are easily accomplished in software development environments. The tools providing the most visibility into a design are those that are built into the design, which are at the same time are the most costly to modify.

Software debugging environments are considered the gold standard of debugging. It is at all times possible to inspect any variable and stop the application at any arbitrary location under a variety of conditions. Debugging conditions, such as breakpoints and any conditions that trigger them, can be changed instantaneously without recompilation. A model similar to this is desirable for FPGAs. 
There are two primary obstacles to build a transparent and flexible FPGA debugging framework. First, FPGAs do not present a consistent or standardized internal architecture. While ordinary computers have a discrete, well-defined memory hierarchy where state is maintained, FPGAs may have a loosely structured memory hierarchy where the state of a single register can be physically distributed throughout the device in an almost random pattern. Furthermore, no two consecutive implementations of the same design are guaranteed to be implemented the same way in the device. Second, FPGAs have no standardized interface. Computers have a console consisting of a keyboard and monitor for interactive work, along with a operating system that presents an abstract interface to the hardware, all of which are absent from FPGAs. And even though FPGAs have an abundance of input and output pins, only the JTAG interface is standardized. JTAG's serial access model is not adequate for quickly inspecting large devices and has not gracefully scaled with increasing device sizes. To counter these obstacles, the three attributes of effective debugging as found in software development environments are described below in the context of FPGAs.

\subsubsection{Improving Visibility}

The flexible nature of an FPGA's architecture inhibits visibility into finished designs, as does the widespread adoption of ASIC development techniques which are not well-suited for FPGAs. The majority of these approaches rely on external tools, which are at a distinct disadvantage. Visibility can be improved through two methods. First, by shifting the debugging perspective from outside the FPGA to inside, direct access to the architecture is simplified. Second, visibility is improved if the FPGA can be accessed like a random access memory. Traditional access methods process the device's state by serially shifting it out in its entirety and then decoding it to reconstruct the original design's register structure. From within, the FPGA's memory structure is randomly accessible in its entirety much like 
a conventional memory. This change in perspective is significantly faster since the entire structure is readily accessible. Figure 4.4 shows this comparison between the serial model and a random access model.
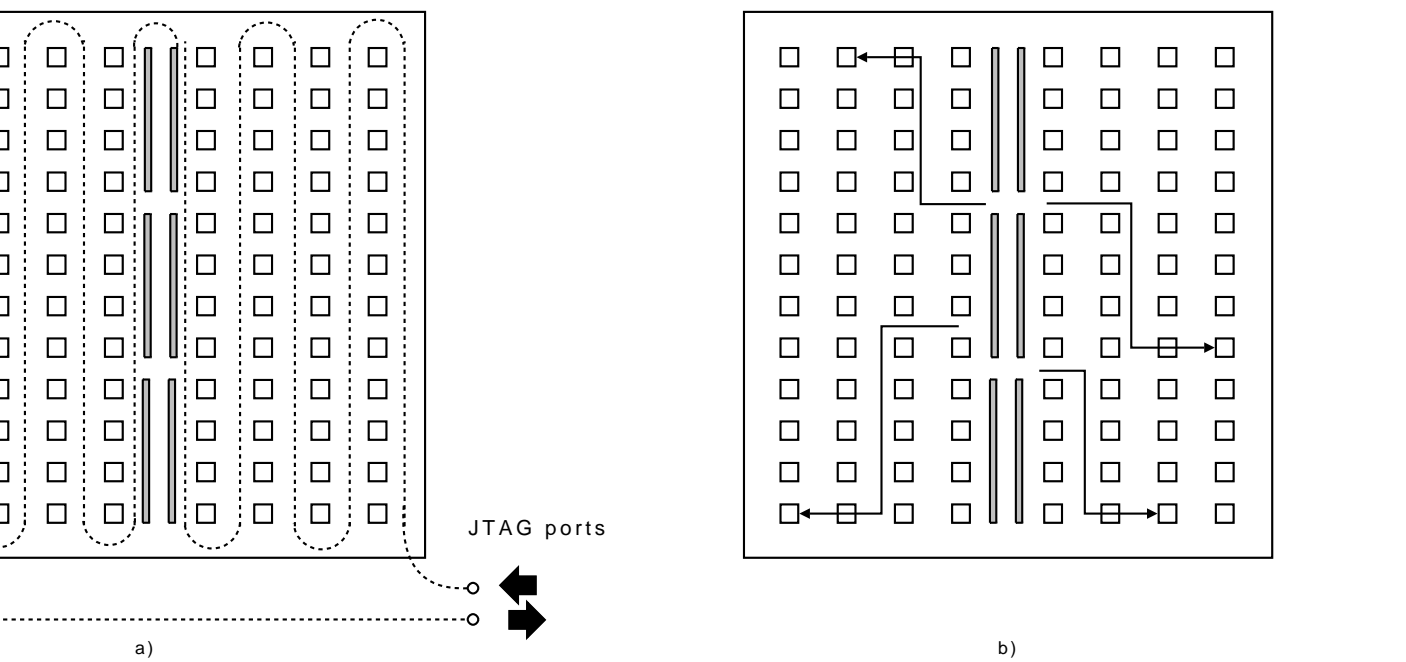

Figure 4.4: a) A serial model of accessing register state; b) a random access model in which registers are accessible as memory locations.

The random distribution of all the design's bits requires additional steps in constructing a mechanism to resolve them. In software, a symbol table resolves an identifier to a memory location. In debugging environments, the symbol table is expanded to include the symbol's name as it appears in the source code along with additional information such as size or data type. A similar symbol table must be built for the purposes of resolving each register's bit location so that the original register can be accurately reconstructed within the debugger.

The construction of a symbol table for an FPGA requires additional effort compared to symbol tables for software applications. Unlike a conventional computer where the details of the underlying hardware are not proprietary and standardized through interface conventions such as Hardware Abstraction Layers (HAL) and operating system Application Programming Interfaces (API), each device family is structured differently and each design turn produces a different implementation. A combined model of both the FPGA's architecture and of 
the design is needed. Unlike memory locations in software applications, an FPGA register does not have a single address. Instead, individual bits are distributed across the FPGA's logic resources. Vendor tools must be manipulated in order to produce a mapping of design registers to bit locations within the FPGA's architecture. This mapping is illustrated in Figure 4.5 where the symbol table individually maps each bit of a register to a physical location within the FPGA.

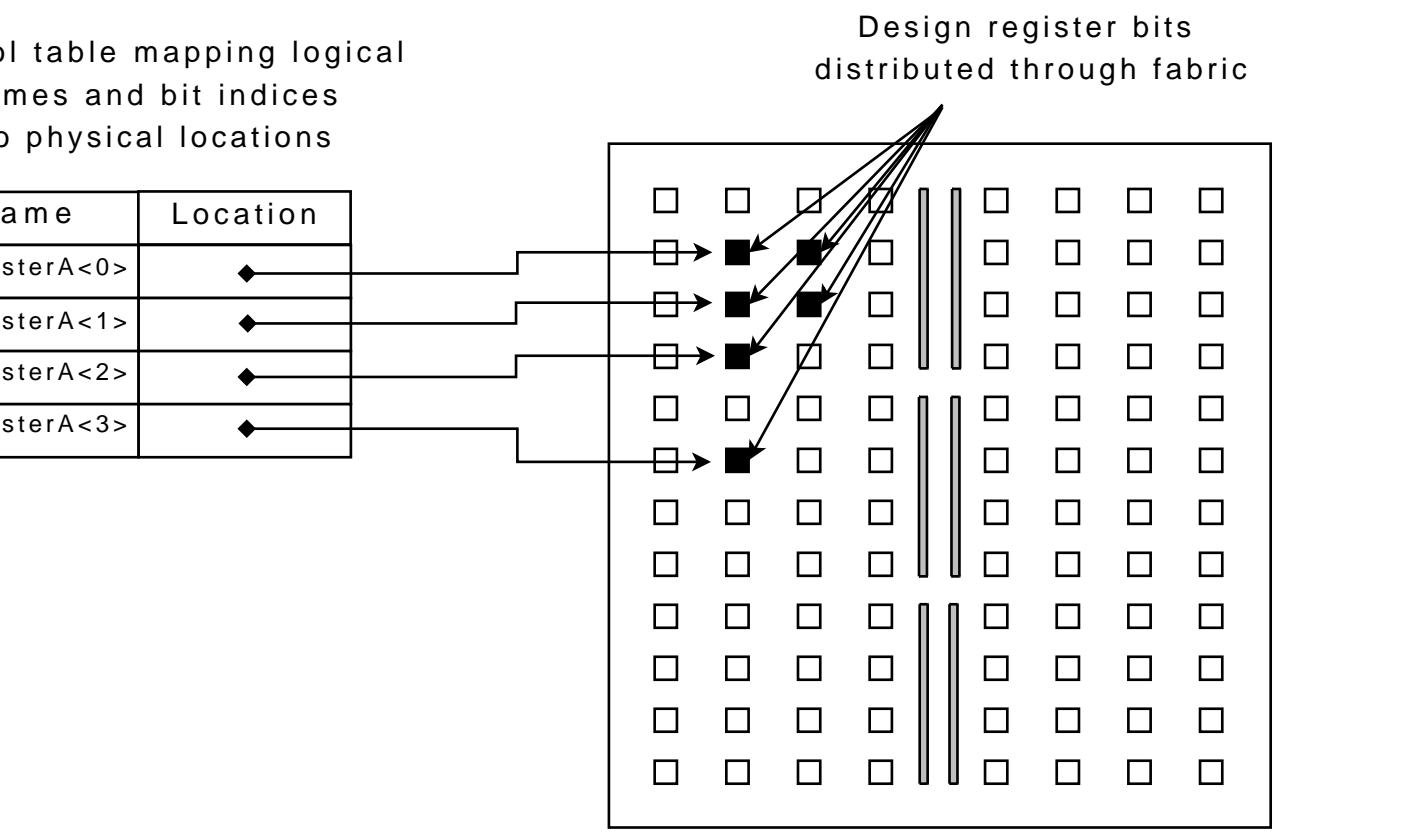

Figure 4.5: Symbol table mapping logical design elements to physical locations in an FPGA.

\subsubsection{Improving Controllability}

Controllability was previously defined to be the extent to which a design's state can be manipulated or altered during execution. In the context of debugging, this relates specifically to how precisely execution can be controlled. Examples of controllability include starting or stopping the execution of a design, advancing a precise number of steps, continuing execution, and defining conditions by which to suspend execution through the use of breakpoints or assertions. 
In comparison to software which executes serially, hardware is a globally concurrent process: designs can be created so that many portions are simultaneously active. As such, the definition of a breakpoint solution in hardware is not defined in terms of an instruction counter as it is in software, but rather on the overall state of the design. Additionally, a hardware breakpoint mechanism must be instantaneous so that the design's state is preserved for inspection. If not, the overall state may change so significantly in a single cycle as to make any diagnosis or inspection impossible.

The most productive hardware design evaluation occurs when execution occurs at the target frequency. Some debugging frameworks allow the state to be captured at each clock cycle, however execution must be slowed considerably for this to occur. The primary concern with this approach is that timing issues can be masked since they will only occur once the design is executing at full-speed. Even a modest $100 \mathrm{MHz}$ clock rate is sufficient to cause timing problems given the complex routing architectures of FPGAs. Other issues arise when certain resources require a minimum frequency for reliable operation, such as video processing components or digital clock managers. A successful control mechanism should not only allow execution to run at the design's target frequency, but be able to halt and hold the design in its current state. The maximum response time to switch from full-speed operation to a halted state must be less than a single clock cycle. Additionally, the design must be reliably held in a steady state and be able to resume execution without introducing unreliable or noisy transitions which can corrupt logic state, known as glitches.

Clock buffers allow clocks to be reliably controlled without introducing such glitches. Attempts to manage clock signals with ordinary logic results in gated clocks. Gated clocks occur when clock signals are handled as ordinary logic. Gating clocks is permissible in ASIC design, but is normally an undesirable condition in FPGAs. Unpredictably latency and loss of clock signal integrity are two risks, since the FPGA's normal signal routing architecture 
Table 4.1: Truth table for a simple clock buffer.

\begin{tabular}{|l|l|l|}
\hline \multicolumn{2}{|c|}{ Inputs } & Output \\
\hline In & ENB & Out \\
\hline $\mathrm{X}$ & 0 & 0 \\
\hline $\mathrm{I}$ & 1 & $\mathrm{I}$ \\
\hline
\end{tabular}

is not intended for clock signals which require special handling. Gated clocks are commonly detected by FPGA vendor tools and warnings are issued. Clock buffers can have several control inputs, clock inputs, and a clock output whose source is determined by the control lines. The most common uses of clock buffers are to reliably distribute clock signals to a large number of components (also referred to as high fan-out,) allow for glitch-free transition between different clock sources (for example, transitioning from a high-speed to a low-speed clock to reduce power consumption), or transition between an active clock and a constant signal which safely halts the connected components. The schematic symbol for a simple clock buffer is shown in Figure 4.6. The truth table for a clock buffer is given in Table 4.1 and shows that while enabled, the buffer passes the input clock directly to the output port, but when disabled holds the output line at a constant logic level. Clock buffers are not simply logic gates reserved for clock signals, but rather a specialized component for reliably distributing clock signals and are frequently capable of being configured to the application. For instance, clock buffers can be configured to pass logic high or low when enabled. Additionally, clock buffers have logic that coordinates the transition of the signal based on its inputs.

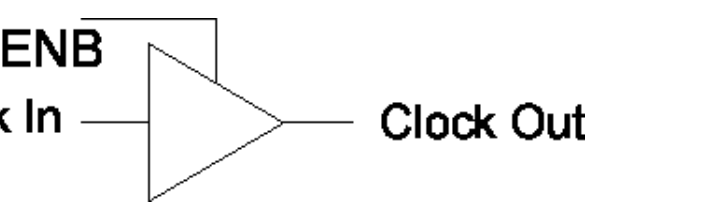

Figure 4.6: Schematic representation of a simple clock buffer. 
Clock buffers are central to an effective control strategy. The most frequently used operations that define controllability center around execution. Operations such as running, stopping, and stepping are transferable to a hardware platform through the use of a clock buffer that sources the master clock of the design. With a clock buffer ensuring glitch-free transitions, execution control becomes a matter of developing a means of integrating the controlling factors, such as interactivity and breakpoint management.

Breakpoints are the final element to controllability. Software breakpoints are easy to write, typically a simple command followed by a location and optionally a condition. Their usability also stems from their conditional syntax being in the native language. Since source code location is not applicable to concurrent hardware designs, asynchronous, conditional breakpoints become the basis for a hardware-based debugger. While synchronous logic limits the complexity that can be implemented, asynchronous logic can exactly satisfy Boolean conditions of its inputs and outputs and more importantly immediately raise a signal without requiring an additional clock cycle as is the case with synchronous logic.

\subsubsection{Improving Agility}

The final attribute for productive FPGA debugging is agility. Agility is defined to be the ease and efficiency at which modifications can be made to a debugging configuration. In software debugging, breakpoint modification is effortless. With graphical user interfaces, the breakpoint and any associated condition is annotated in the source code or listed in a separate window. Even with command-line based debuggers such as the GNU gdb debugger, breakpoints can be quickly specified with a single command and a line number, file name, or function name. FPGA logic analyzers are the closest to this model of use, but require more effort to setup and much longer to modify. Modifications to embedded logic analyzers are typically treated like any design modification and may require a full design 
re-implementation. Given that the general toolflow is time consuming and increases with design complexity, it is desirable to isolate any debugging logic from the rest of the design.

By physically separating debugging logic from the rest of the design, the debugging module can be re-implemented multiple times independently of the rest of the design. The debugging module's small size relative to the rest of the design would significantly reduce implementation time. Such a scenario aligns the FPGA debugging environment experience with that of a software debugging environment where relatively short times are required to alter the debugging configuration. To achieve this rapid turnaround and design isolation, partial reconfiguration can be leveraged which allows a region of the FPGA to be reserved for multiple modules that can be swapped in and out on demand. A smaller, isolated region also reduces the implementation time required. This rapid modification is ideal for interactive scenarios, such as breakpoint logic implemented in hardware. Figure 4.7 shows a separate reconfigurable region dedicated to implementing debugging logic. The distinct module allows rapid modifications of the debugging logic without re-implementing the entire design.

\subsection{Dynamic Modular Design and Validation}

The Dynamic Modular Design (DMD) framework envisions Xilinx's PR [67] flow not as a runtime strategy, but a design time methodology. While developers have not widely adopted PR for production designs, the research conducted for DMD has shown that rapid development turnaround times can be achieved by partitioning frequently modified modules into separate PR regions [68]. DMD's use of PR does not extend beyond the development environment since PR regions are gradually and automatically merged out of the design. The PATIS floorplanner will be discussed briefly as it relates to the overall DMD flow, however an

extensive discussion can be found in $[20,37,38,68,69]$. There are two principal components 


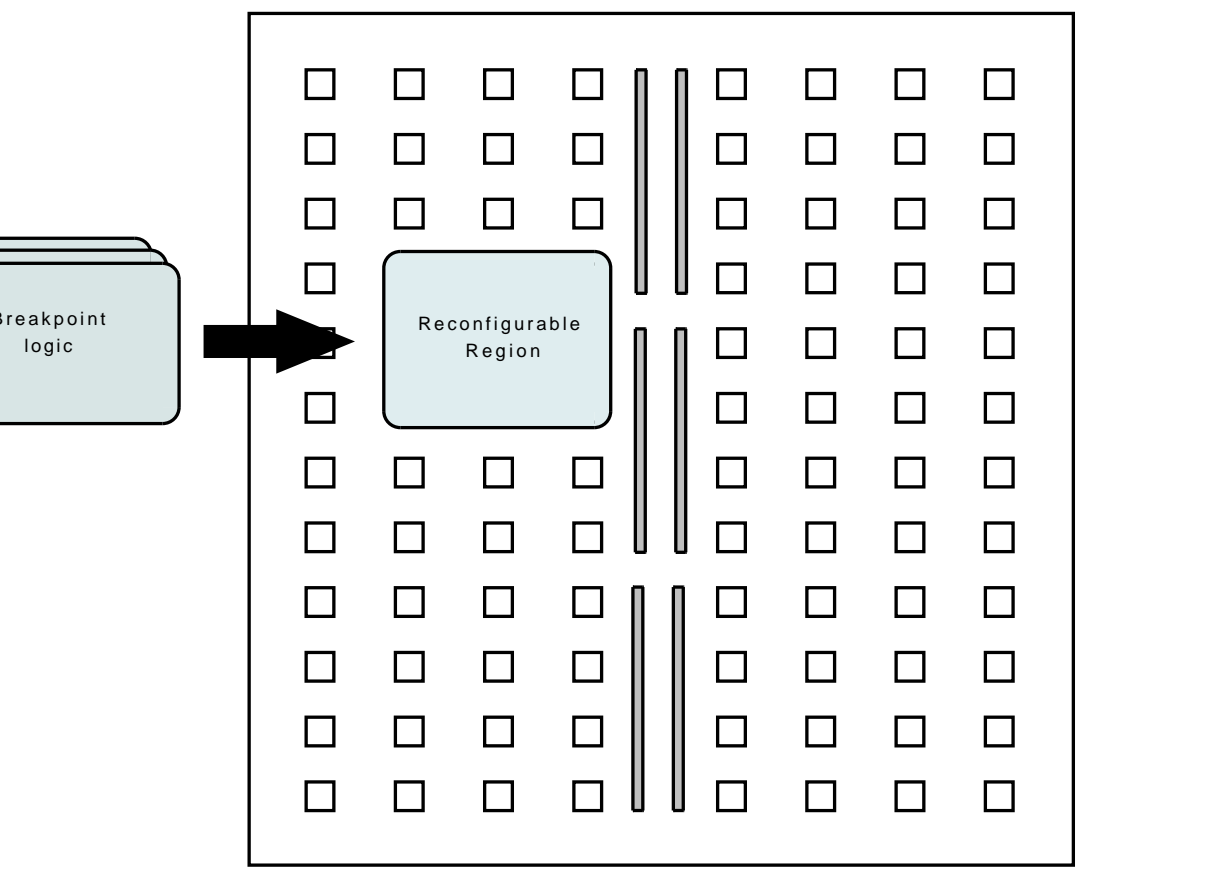

Figure 4.7: Accelerating debugging turnaround with a reconfigurable region for debugging logic.

of DMD: the PATIS floorplanner which is discussed below and the debug validation tools which are the subject of this research.

\subsubsection{PATIS}

DMD extends the traditional PR flow with the Partial module-producing, Automatic, Timingaware, Incremental, Speculative (PATIS) floorplanner [20]. While the standard PR flow has traditionally been a manual process not normally associated with enhanced productivity, the PATIS tools simplify much of the implementation details of PR by automating the process. Since runtime reconfiguration capabilities are not required, PATIS uses a simplified version of the PR flow that does not have the complications found in runtime hot-swapped modules. Bus macros are automatically inserted on module boundaries and provide passive, readback-based observability of all communication between floorplanned modules [37]. 
The primary obstacle to fast implementation times for FPGAs is place-and-route (PAR). While vendor tools implementing parallel PAR algorithms and leveraging the lower-cost of multi-core processors have been introduced, they have yielded only a modest speedup due to the complexity of the algorithms and the large, complex data structures which saturate memory bandwidth and overrun caches. PATIS counters these obstacles through a "divideand-conquer" strategy that creates independent floorplan variants of the design that are parallelizable on separate machines and do not require shared memory access. By separating modules that are currently under development into independent PR regions, modules can be separately implemented without reimplementing the entire design, dramatically reducing implementation times.

PATIS defines two attributes to describe maturity, fickleness and viscosity, and uses these to determine when to migrate modules from the reconfigurable regions to the static area. Fickleness is characterized by the effort required for a module to meet timing requirements, while viscosity is defined by the frequency of historical changes to the module which may provide indicators of the likelihood that it will change again.

The goal of PATIS is to recast partial reconfiguration not as runtime strategy, but as a designtime methodology for static designs. Separate reconfigurable regions for each module, as shown in Figure 4.8, enable a unique feature of PATIS: the ability to swap a previous revision of a module out for a more recent one without the need for rebuilding the entire design. This is similar to linking in software where modified files are recompiled and inserted into library archives and then linked to executables without recompiling the entire application. The time-savings can be significant.

The PATIS floorplanner creates a modestly oversized PR region for each top-level module allowing modules to grow during the course of development without disturbing the rest of the design. If an updated version of a module no longer fits within its boundaries, PATIS selects 


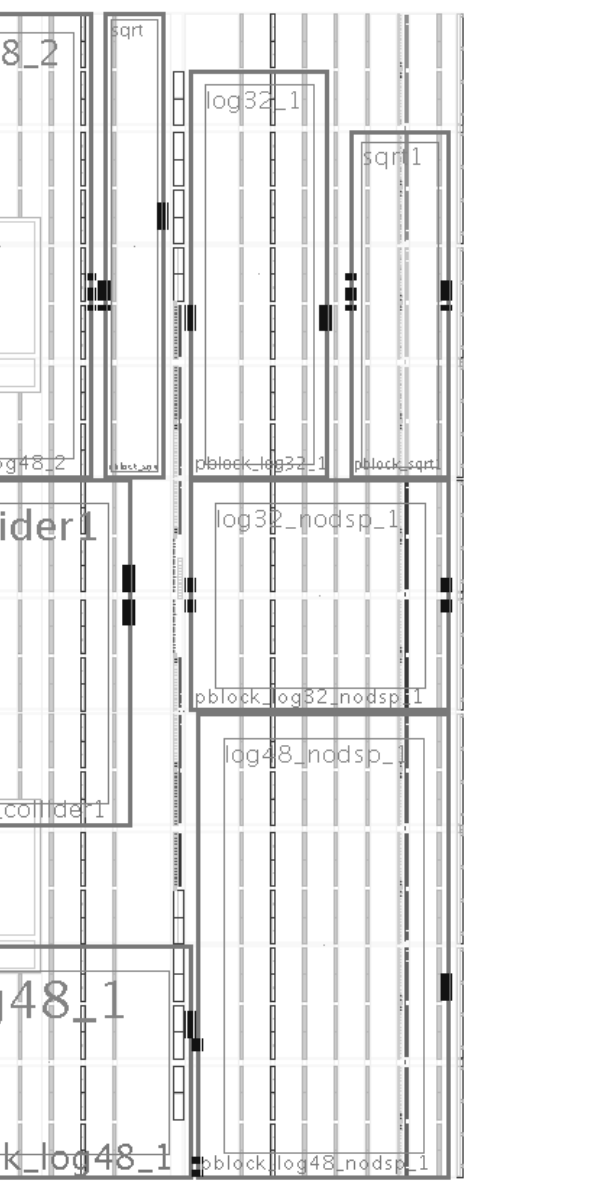

Figure 4.8: PATIS provides each top-level module its own reconfigurable region.

an appropriate floorplan from a database or re-implements the entire design. A speculative floorplanning background process runs that explores the design space, generating potential future floorplans based on estimated module completeness and past changes. Timing is analyzed across module interfaces and compared to top-level constraints. A thorough discussion of PATIS can be found in [68].

Figure 4.9 illustrates the DMD flow. Whenever a module change affects the design floorplan, PATIS tries to accommodate the changes by applying a minimum set of updates to the existing floorplan. Ripple effects are considered, and a completely new floorplan may be generated if incremental changes are inadequate. The speculative floorplanning background 


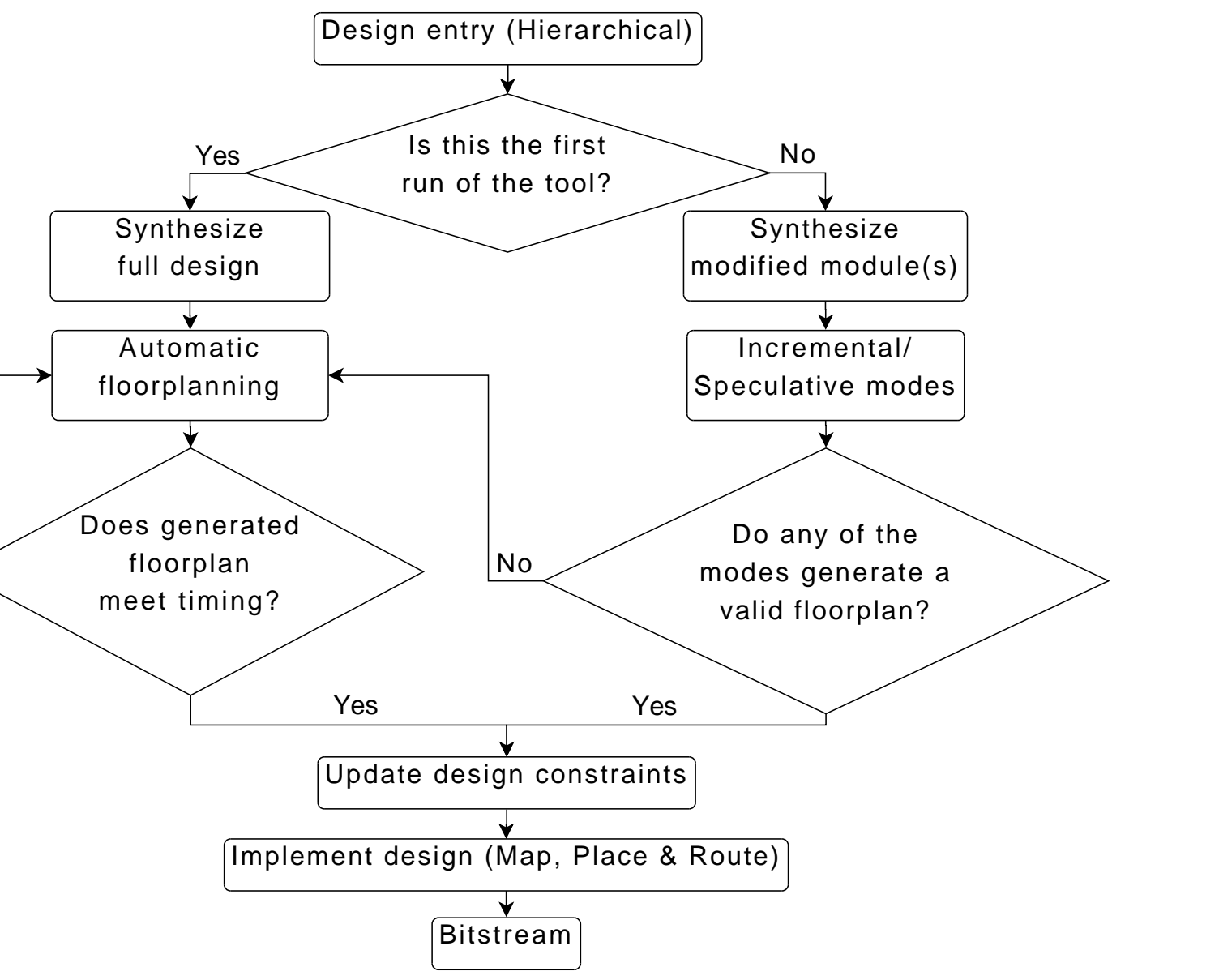

Figure 4.9: Dynamic Modular Design flow.

process populates the database with design space explorations of potential floorplans based on past changes. Floorplans include an optimized placement of bus macros. Timing is analyzed across module interfaces and compared to the top-level constraints.

Implementation acceleration comes from the reduction of a large global optimization problem to a set of smaller, independent problems. Optimization restrictions across module boundaries are generally accepted for the sake of design productivity and timing closure. Although the PATIS/PR flow adds bus macro overheads to inter-module routing, the use of registered bus macros may improve system clock frequency when critical nets span module boundaries. 


\subsection{Summary}

This chapter explored some of the key concepts necessary for developing a validating and

debugging framework. HLV seeks to tie the reference model created during the first stages of development to a functioning hardware implementation. This not only functionally validates the hardware, but additionally allows increases to testing productivity since the hardware is now under software control. HLV can assist even during later stages of development by serving as unit testing framework for individual hardware components. At the other end of the development process, LLD addresses the three attributes of low-level FPGA productivity: controllability, visibility, and agility. Methods for establishing fine-grain control over a design, repositioning the debug perspective to allow uninhibited access to the internal structures, and a means for mirroring the breakpoint model found in software were discussed. Finally, the DMD project was summarized including an overview of the PATIS floorplanner which enables the debugging framework to coexist in the rapid design turnaround environment created through partial reconfiguration. 


\section{Chapter 5}

\section{Implementation}

Chapter 4 presented two approaches to improving FPGA developer productivity at different phases during the development cycle. The HLV framework seeks to bridge the model verification gap by providing a means of directly linking the initial high-level reference model to implemented hardware. The LLD framework is applied during the later stages of development by applying fine-grain control over the execution and providing detailed insight into an implemented design's state. Both these frameworks derive their use model from the debugging environments long prevalent in software. Chapter 5 presents the implementation details of these two frameworks.

\subsection{High-Level Validation}

HLV addresses the model verification gap wherein the reference model produced during the initial development stages becomes isolated from hardware development. HLV provides automated functional validation of synthesized hardware by binding it directly to the original, untimed reference model. The resulting framework can be applied to hardware much like 


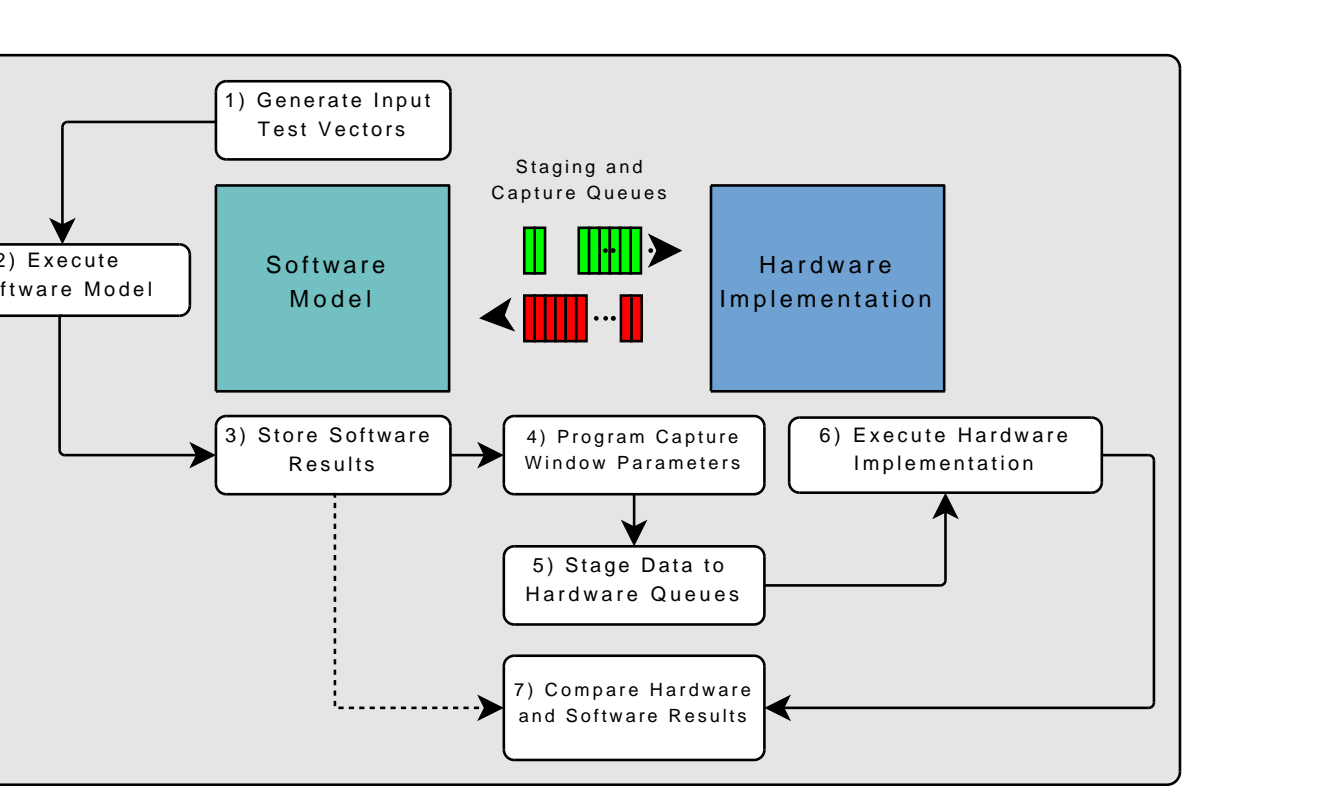

Figure 5.1: Overview of High-Level Validation.

unit testing frameworks are in software for frequent testing, such as nightly or regression testing. HLV is a hardware/software framework based on capture methodologies similar to those found in embedded logic analyzers. Since HLV is targeted towards automated tests, the resource overhead of capture techniques is considered acceptable. Figure 5.1 outlines the HLV framework which consists of an on-chip processor with a peripheral that functions as a test harness for the module. The peripheral provides input and output ports for data and control signals, queues for staging input data as well as capturing output data, and the logic for controlling the capture. The microprocessor provides a platform for the software-based testbenches, a user-interface over a serial console, as well as a platform for executing the reference model. Input data is distributed to both the software model and the hardware implementation, executed on both, and the results compared for consistency.

HLV can be used as a nightly, software-controlled, hardware unit-testing framework. Simulation data provides the model with control signal interactions and latencies to develop the software testbenches. As discussed in Section 4.1.2, control signals found in hardware 
cannot be mapped to the corresponding software model. The HLV testbench software can provide software control of designs during development and debug, scanning the output for expected data produced by the software model, and reviewing output streams without re-implementing the design as with conventional hardware debugging. Software-controlled testbenching has rapid turnaround times and requires no hardware re-implementation to inspect different parts of the design, output streams, or to test different scenarios. Elaborate, software-generated unit tests can be created for each module, enabling hardware validation against the reference model. The cost for changing a testbench is no longer the time required for hardware re-implementation, but rather the time required to recompile and reintegrate the software into the hardware bitstream.

\subsubsection{Reference Model Execution and Hardware Data Staging}

HLV operates by first executing the software reference model on the microprocessor using prepared or randomly generated input data and the storing the results for that data set. Next, the same input data is staged in the input queues of the test harness. Control signal interaction and latencies gathered during simulation are programmed using HLV's API. The same API is then used to configure the capture window which defines the clock cycle range of the output data to store for comparison to the reference model. Output data that lies outside the capture window range is discarded. The use of queues for input and output data enable the design to run at its target operating frequency without having to develop a technique to control the execution of the design.

HLV uses a processor to allow users to enable programs to interact directly with the hardware to be tested. The processor provides a platform for the HLV driver software, the software testbench, and enables communication over a serial console. The Xilinx MicroBlaze processor was chosen for its small size, large collection of peripherals, and ease of customization. The 
MicroBlaze is a configurable soft-processor especially designed for Xilinx FPGAs and includes a full C-language GNU gcc compiler. The MicroBlaze and its peripherals are specified with the Xilinx Embedded Development Kit (EDK), a graphical user interface used to build embedded systems on FPGAs. A wide range of peripherals, including memory controllers, UARTs, busses, and custom co-processors can be specified and configured. The processor project created in the EDK can then be instantiated into a higher-level project, such as into Xilinx's ISE, as an ordinary HDL component.

No invasive modifications to the reference model are necessary in order to integrate HLV into the DMD development cycle. The intent of HLV is to create a transparent means of linking the reference model to synthesized hardware without the need to significantly modify either. The testbench software applications to be run on the processor can be rapidly integrated into the bitstream through the use of special vendor tools which initialize internal BRAMs. Software compilation and reintegration with the bitstream can be done with a single command from within the EDK.

\subsubsection{Test Harness and API}

The test harness is a custom MicroBlaze peripheral that connects the processor to the hardware module to be validated. The test harness consists of input/output pairs of First In, First Out (FIFO) queues which connect the hardware component directly to the data path of the processor, enabling the DUT to operate at the target frequency. The test harness also provides logic which controls the capture process.

Through its Fast Simplex Link (FSL) architecture, the MicroBlaze provides direct access to its data path through special instructions and ports $[24,70]$. FSL is a configurable length, 32-bit wide, point-to-point, unidirectional FIFO-based processor interface where data can be queued for reads or writes at high-speeds, allowing streaming data peripherals to directly 
interact with the MicroBlaze's data path. A low-overhead interface such as FSL reduces the overhead and complexity between the data path and the DUT. This is preferable to interfacing through a bus-based peripheral which adds additional hardware and software overhead and thereby operates slower.

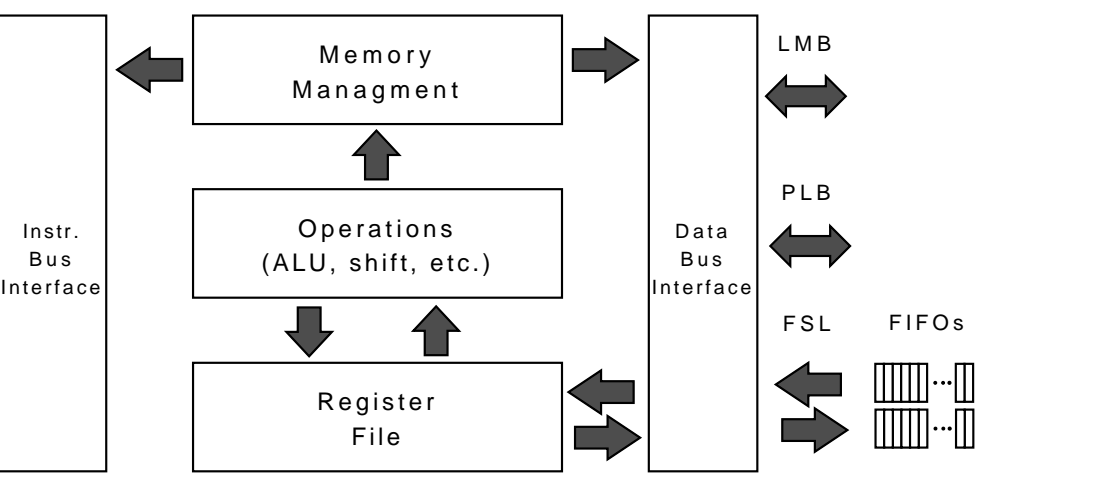

Figure 5.2: MicroBlaze architecture.

Figure 5.2 shows that unlike other busses available on the MicroBlaze, such as the PLB and LMB interfaces which connect to intermediate hardware peripherals and requires driver software, the FSL connects directly to variable length FIFOs. For this reason, FSL is the preferred choice for interfacing with custom hardware accelerators. For even higher performance, FSL can be configured to use BRAMs rather than distributed RAM implemented in ordinary configurable logic. Data can be handled deterministically, with latencies for sending or retrieving data to and from the interfaces at one to two clock cycles, depending on the configuration [24]. The MicroBlaze includes FSL instructions as part of its binary interface, further reducing overhead and latencies. Furthermore, FSL instructions are available in blocking and non-blocking variants which can halt execution when FIFOs are full or empty. These options could be used to develop complex, long-running testing scenarios.

Use of the Xilinx EDK enables peripherals to be quickly added to a MicroBlaze. Known as co-processors, a short series of configuration options can be set in the graphical user interface which produces a co-processor stub connected to a MicroBlaze. The stub is generated 
Table 5.1: Summary of HLV API.

\begin{tabular}{|l|l|}
\hline Function & Description \\
\hline \hline write_fsl_data & Write data to the FSL data queue \\
\hline write_fsl_ctrl & Write control data to the FSL control queue \\
\hline write_fsl_pair & $\begin{array}{l}\text { Write data and control data to the corre- } \\
\text { sponding FSL queue in unison }\end{array}$ \\
\hline hold & $\begin{array}{l}\text { Hold control and data at a constant value for } \\
\text { the given number of clock cycles }\end{array}$ \\
\hline
\end{tabular}

with instructions on how to customize the HDL to produce the co-processor, as well as the framework for software drivers to access the new peripheral through memory mapped registers or streaming instructions. The test harness was created as an FSL co-processor and then customized with large-capacity FIFOs to store long streams of data and control signal information for both input and output.

The HLV API enables the FSL queues to be populated with data and control streams identical to those gathered from simulation and includes utility functions to hold individual or groups of signals at constant values or transition after pre-defined intervals. A summary of the API calls along with a brief description of their function is included in Table 5.1. The API can be used to build custom signal interactions, such as reset sequences necessary to set the hardware to a known initial state, before streaming input data from the queues. Once populated with the control and data signals, the API is used to release the signal streams to the DUT.

In addition to queuing data and control streams for the DUT, the test harness also implements its capture logic in hardware for the best performance. As noted in Chapter 3, capture methodologies are common in embedded logic analyzers which are instantiated as part of the design and internally record execution traces. A capture methodology is more appropriate in a test environment where individual components will be individually tested rather than 
as part of the entire design. The resource requirements for capture, most notably on-chip memory, will not compete with the design for resources.

As part of the test configuration, the API is used to program the capture window logic which defines the range of the output stream to store in the test harness' queues. Until execution is triggered, a counter is held at zero until the control register's start bit is activated. Once set, the data stored in the input queues is released and begins streaming to the DUT. As the counter increments, the capture window is activated once the counter is between the range of the start and stop registers. Only during that window is the module's output data stored into the test harness queues and ceases being captured once the counter exceeds the stop register value. The capture window is illustrated in Figure 5.3. The test harness can be monitored through the API by a status register, while a control register allows the test harness to manipulated. Table 5.2 shows the memory map of the HLV peripheral's configuration registers.

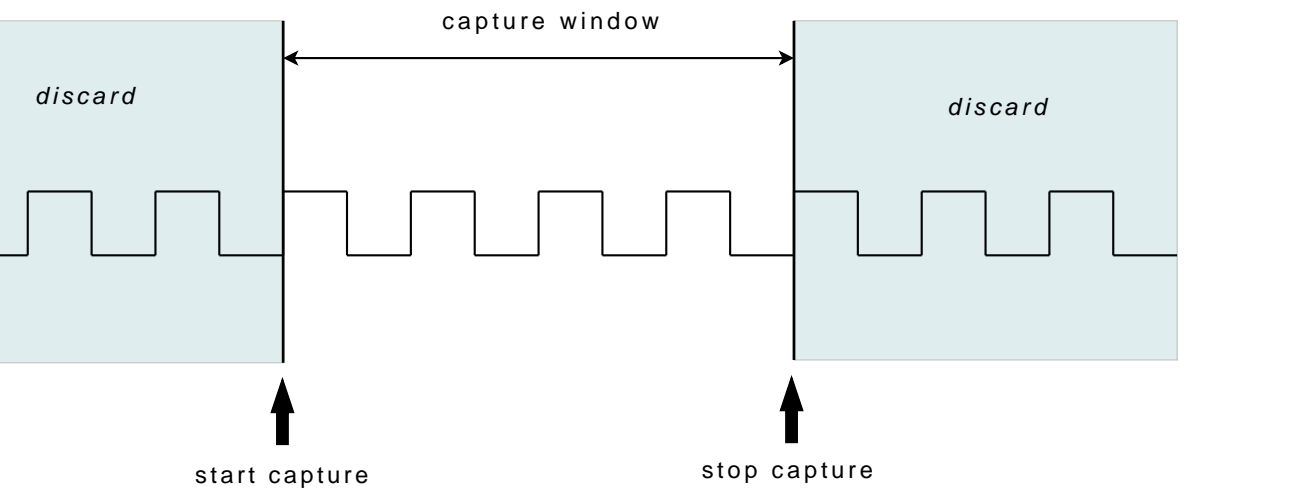

Figure 5.3: HLV capture window parameters.

HLV is unique in that execution occurs at the frequency of the design and does not require that execution be halted or occur at a slower frequency. The DUT is continuously sourced from the system clock. Since HLV can stage data that is to be streamed at full-speed, the DUT can continuously execute even without valid data at its inputs. Placing control sequences, such as reset sequences, ahead of test data in the queues allows the the DUT 
Table 5.2: Register map for the HLV peripheral.

\begin{tabular}{|c|l|l|}
\hline Register & Name & Description \\
\hline \hline 0 & Control register & $\begin{array}{l}\text { Start, stop, and reset functionality for } \\
\text { test harness and queues }\end{array}$ \\
\hline 1 & Start capture & Capture window start boundary \\
\hline 2 & Stop capture & Capture window stop boundary \\
\hline 3 & Status register & Test harness and queues status register \\
\hline 4 & Unused & \\
\hline 5 & Unused & \\
\hline 6 & Unused & \\
\hline 7 & Unused & \\
\hline
\end{tabular}

to be placed into a known initial state and executed as if run in a full execution life-cycle. Operational data extracted from simulation, such as the end-to-end latency of any pipelines, is necessary to ensure that programming of the queues is done properly. This is particularly important for properly resetting the device before execution.

The simplified example program given in Listing 5.1 shows how a software function that has been mapped to a hardware module is executed and its data stored. The same data is then paired with control signals extracted from simulation and placed into the data and control queues. The start and stop registers defining the capture window are then set before the hardware module is set to execute. The output data is then captured and automatically compared to the software module's results.

\subsection{Low-Level Debug}

The LLD framework aims to introduce the interactivity and abstraction found in software debug environments to FPGAs. Similar to HLV, LLD consists of an on-chip processor controlling a custom peripheral which interfaces to the design. The processor controls the 


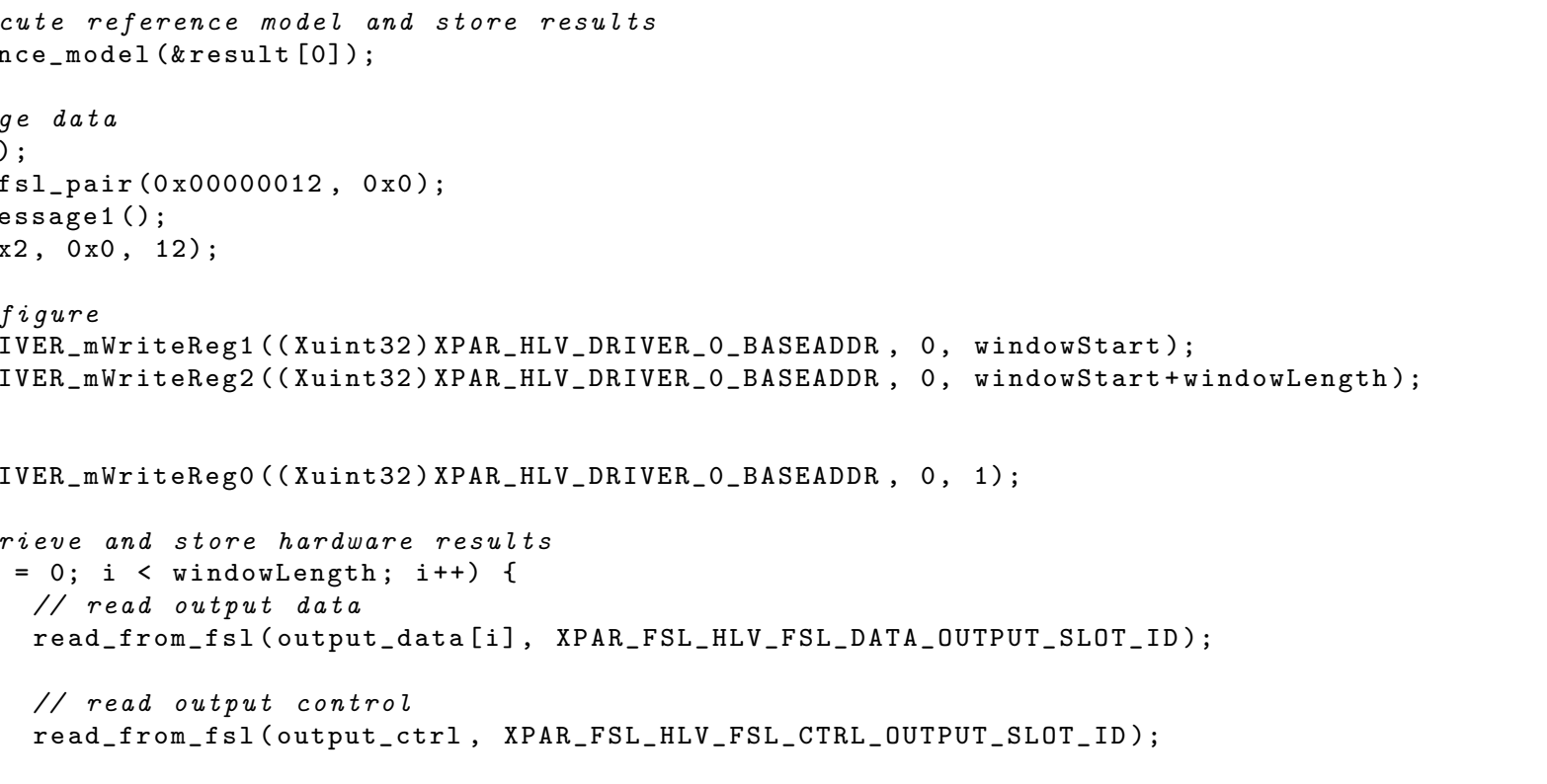

Listing 5.1: Sample HLV program.

execution of the design and provides an interactive, scriptable command-line interface that interacts with a second, workstation-based application. Whereas embedded software running on an FPGA would be too slow and resource-limited to meaningfully interact with a running design, LLD uses the on-chip processor as a resident delegate to provide more visibility and insight from within the FPGA to the workstation application. The custom hardware peripheral provides rapidly interchangeable condition-based breakpoint and assertion capabilities through the use of partial reconfiguration. The breakpoint logic is capable of halting and holding the design at any arbitrary location for inspection. A state readback utility implementing the ICAP interface is capable of reading any design register in the device, treating it as a memory rather than requiring a shift chain. An overview of LLD is given in Figure 5.4. Software models of both the FPGA device and the implemented design provide the information necessary to interact with and interrogate the FPGA directly. However, the resource and processing requirements of such models far exceed the available resources of any current 

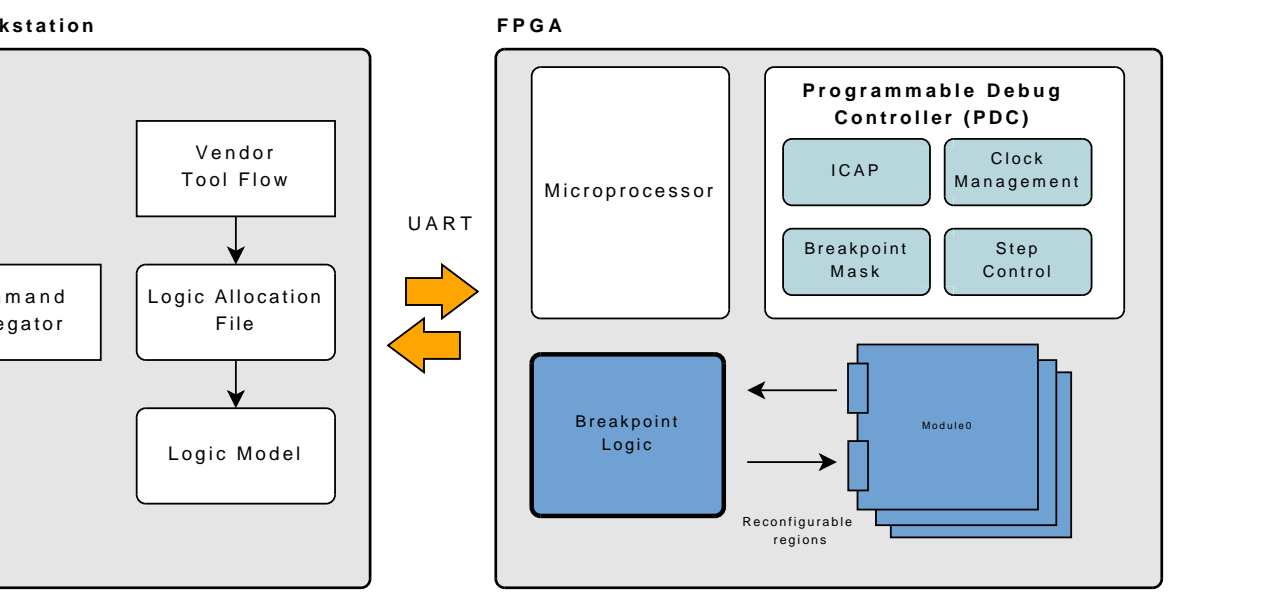

Figure 5.4: Overview of LLD.

FPGA. Therefore, a workstation application is needed to construct the model of the design and map hierarchical design names to individual bit locations in the FPGA. The application then assembles and issues a stream of commands to the on-board processor to retrieve and decode state information through the ICAP. The individual components of LLD are discussed below.

\subsubsection{Programmable Debug Controller}

The Programmable Debug Controller (PDC) is the interface between the processor and the design to be debugged. The PDC is a custom peripheral of the MicroBlaze that manages clock logic, status signals from the reconfigurable breakpoint module, and the ICAP to read state information. The Xilinx ISE EDK has facilities to customize and generate stubs for processor peripherals. Peripherals can be connected directly to the data path through the FSL interface or through a conventional bus such as PLB. The EDK automates the generation of interfaces, drivers, and memory-mapped registers. An API was developed to drive this peripheral using these stubs. Its register map is shown in Table 5.3 while a discussion of the subcomponents follows. 
Table 5.3: Register map for Programmable Debug Controller.

\begin{tabular}{|c|l|}
\hline Register Number & Description \\
\hline \hline 0 & Development and debug register \\
\hline 1 & Development and debug register \\
\hline 2 & Unused \\
\hline 3 & Unused \\
\hline 4 & Unused \\
\hline 5 & Unused \\
\hline 6 & Unused \\
\hline 7 & Unused \\
\hline 8 & Unused \\
\hline 9 & Unused \\
\hline 10 & Unused \\
\hline 11 & Active breakpoint mask \\
\hline 12 & Step counter target \\
\hline 13 & Unused \\
\hline 14 & Breakpoint enable mask \\
\hline 15 & Control register \\
\hline
\end{tabular}

\section{Clock Management}

The PDC's clock management unit enables fine grain control of the design's execution. Clock buffers, which were introduced in Section 4.2.2, enable glitch-free transitions between clock sources including sources that are being held at a constant logic level. Using the PDC's software interface, the design can be run freely at its targeted design frequency or stepped an arbitrary number of clock cycles, also at the target frequency and duty cycle. At startup, the clock is disengaged and the design is halted until manually started from the commandline either through a run or step command. It then executes until either a breakpoint or assertion condition occurs, a user-issued command to stop is given, or if the programmable step counter expires. Figure 5.5 shows an overview of the LLD's clock control and control unit. Hardware-controlled clock stepping, rather than a software-generated clock signal, allows the design to be reliably stepped a predetermined number of cycles using the actual 


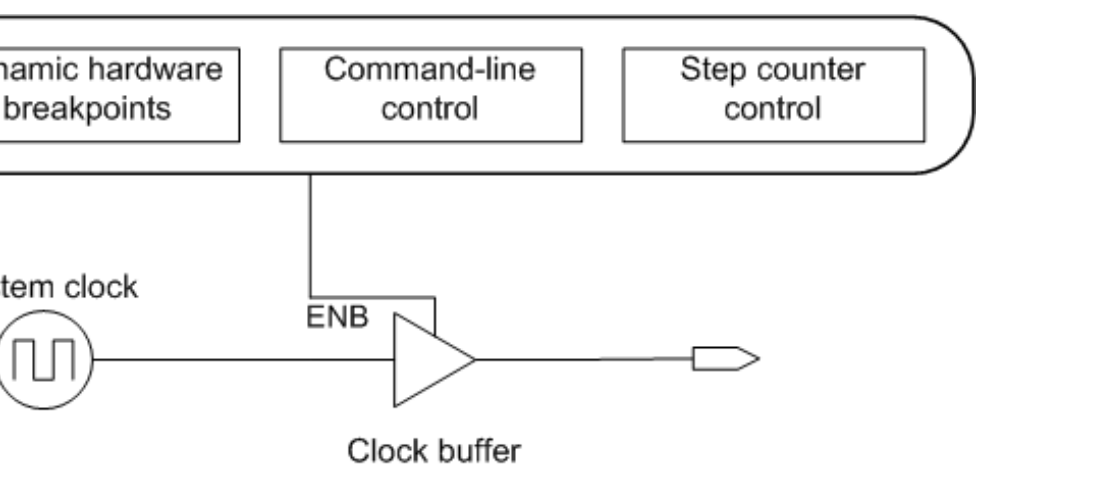

Figure 5.5: LLD clock control.

system clock which eliminates the introduction of timing and transition glitches. The clock management architecture can be extended to cross multiple clock domains by cascading clock buffers.

The PDC halts the design by enabling the clock buffer, which then holds the output clock line at a constant logic high level. Clock buffers differ from conventional buffers by taking into account rising and falling edges of their sources and deferring transition from one source to the other until the transition has completed on both sources, thereby avoiding the introduction of glitches. The clock buffers used in the PDC have a single clock source, ensuring that the reaction time is consistently one clock cycle which is sufficient to halt the design for debug purposes.

\section{Dynamic Breakpoint and Assertion Logic}

An agile and interactive breakpoint management strategy is a novel contribution of this work. Up to 32 software-addressable breakpoints sourced from top-level signals can be programmed into a dedicated, reconfigurable breakpoint region managed from the user console. Support is provided for two types of breakpoints: conventional, conditional breakpoints that suspend execution when a condition is met, and assertion-style breakpoints that suspend execution once a condition fails to be met. Assertions for simulation-based development were 
introduced in Section 2.3.4. Unlike conventional assertion-based verification, the assertions found in DMD are written in conventional Verilog and respond like software assertions which interrupt execution immediately. Breakpoints can be modified and quickly re-implemented into hardware without re-implementing the entire design, achieving significant time savings especially for large or high-utilization designs. Breakpoint statements can also be selectively enabled or disabled from the command-line through a software-controlled breakpoint mask. The 32 individual bits of the breakpoint mask register each correspond to single breakpoint statement. The individual bit of the mask is logically AND'ed with the signal from each breakpoint, allowing any breakpoint statement to be temporarily disabled and then re-enabled as needed. Breakpoint logic is implemented as asynchronous logic which signals its activation immediately and causes the clock buffer to suspend design execution at the next rising clock edge. This ensures that the design is held in the precise state that caused the breakpoint to trigger. Immediate suspension of execution improves the possibility of finding the underlying cause of an assertion failure or inspecting the design in the state that caused the breakpoint to occur. A detail of the breakpoint region is shown in Figure 5.6. Breakpoint logic is maintained in a separate, top-level, partially reconfigurable region. Implementing the debug region as a top-level module exposes the interface to the design's top-level signals and ports without necessitating changes to intermediate module interfaces. A PR region allows the breakpoint logic module to be swapped out in a matter of seconds without interrupting the execution of the design or needing to reset its state each time. Toplevel signals were chosen as the most versatile means of monitoring the design since these are most likely to reflect the global state of the design.

Breakpoint tables consist of 32 asynchronous breakpoint logic conditions and reference toplevel signals or ports. The dependency on top-level signals does not limit the usability of the framework since internal state is usually validated through simulation prior to implementation and the actual state of any arbitrary register can be inspected through the PDC's ICAP 


\section{Breakpoint Table}

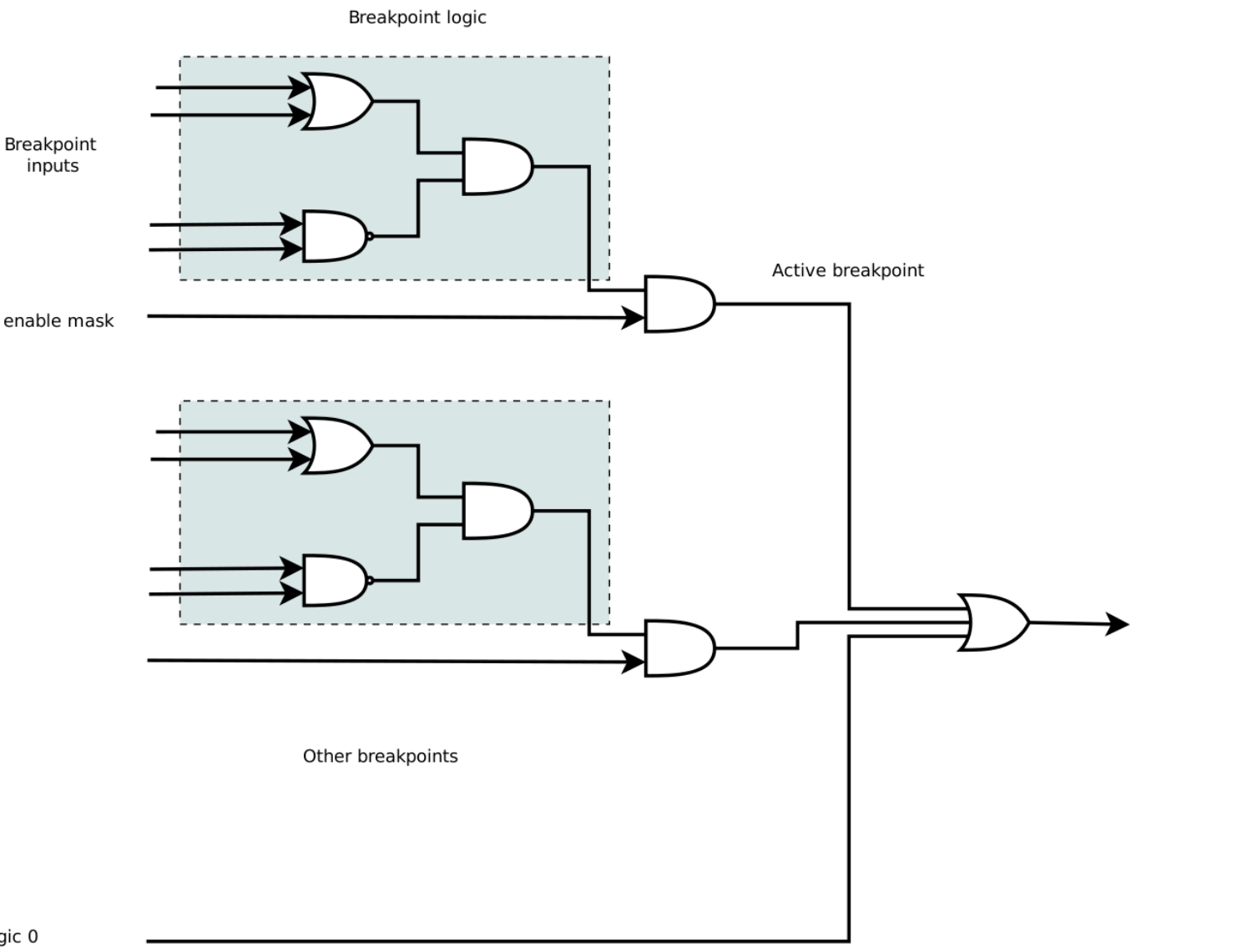

Figure 5.6: LLD breakpoint region detail.

interface once the design has been halted. An overview of the ICAP interface was given in Section 5.2.1.

Breakpoints are implemented as asynchronous logic to allow fast, single-clock cycle response times that suspend execution at the next rising clock edge. Breakpoint conditions are entered from the workstation application console as valid Verilog and can be a combination of any top-level signals or ports previously defined as the interface to the breakpoint region. If signals other than those previously selected are needed, a full re-implementation of the design is necessary as discussed in Chapter 1 to reroute signals and re-establish the 
breakpoint module interface. Breakpoints unconditionally suspend execution of the design and must either be disabled through the breakpoint mask or have the activating condition changed in order for execution to resume. Software-based breakpoints were considered and would have eliminated the dedicated routing as required for this implementation. While software-based breakpoints would have created a far more agile and sophisticated breakpoint strategy, execution time would have suffered as the entire array of monitored signals and their associated conditions would need to be repolled at each clock cycle, eliminating the possibility of running the design at its target frequency.

The workstation application manages the breakpoint table that is later synthesized into the breakpoint logic module. An array of breakpoint structures, given in the Listing 5.2, is held in memory and regenerated as a Verilog module after every modification. The breakpointText field contains the original breakpoint statement and must have signals which correspond with the previously declared port names of the breakpoint logic module. Once entered, breakpoint conditions are written out to a Verilog module and processed with the partial bitstream tool flow.

The generated source of a sample breakpoint module is given in Listing 5.3. Empty breakpoint table entries are assigned a constant logic 0 to prevent them from interfering with the breakpoint logic even if the corresponding breakpoint mask is accidentally enabled. There are no control signals into the breakpoint module, only design signal inputs. Likewise, there are only two control outputs from the breakpoint region: an aggregate line to indicate an active breakpoint, and a mask showing the index of active breakpoints. The aggregate line is intended to signal an interrupt to the MicroBlaze when the status of any breakpoint changes to cause an interrupt routine to automatically print information about the active breakpoint. Given the small size of the breakpoint region, the resulting module is fast to implement into hardware and requires a nearly insignificant number of resources. 


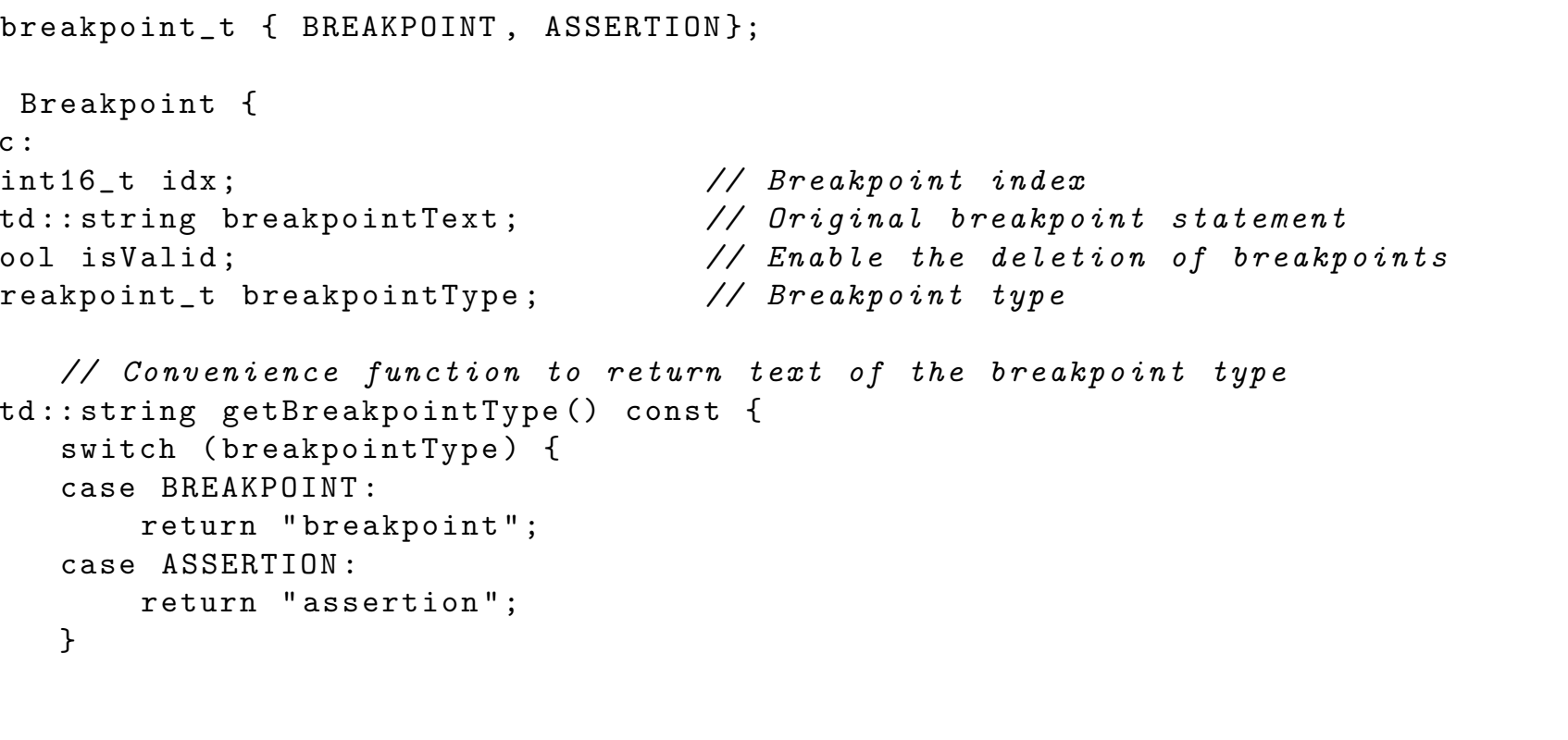

Listing 5.2: Breakpoint data structure.

\section{"Inverted" Bitstream}

The increasing size and complexity of FPGAs warrants that extra precautions are taken with the implementation flows. Design rule checks (DRC) are repeatedly enforced throughout the implementation cycle ensuring that the device is not configured in a manner that would somehow physically damage the device, for instance a logic high line tied directly to ground or two different drivers on the same line. DRC is performed on the entire design, regardless of how small the change is or if even just a partial region is re-implemented. The entire design is processed for checks such as DRC, and the time required increases proportionally with the design size. For large designs on large devices, the overhead of this DRC can be significant.

To counter the lengthy runtimes that would result in regenerating a debug module, an "inverted" bitstream was devised. Inverted bitstreams refer to the replacing the logic outside the reconfigurable debug region with an empty design, while still preserving the boundaries 


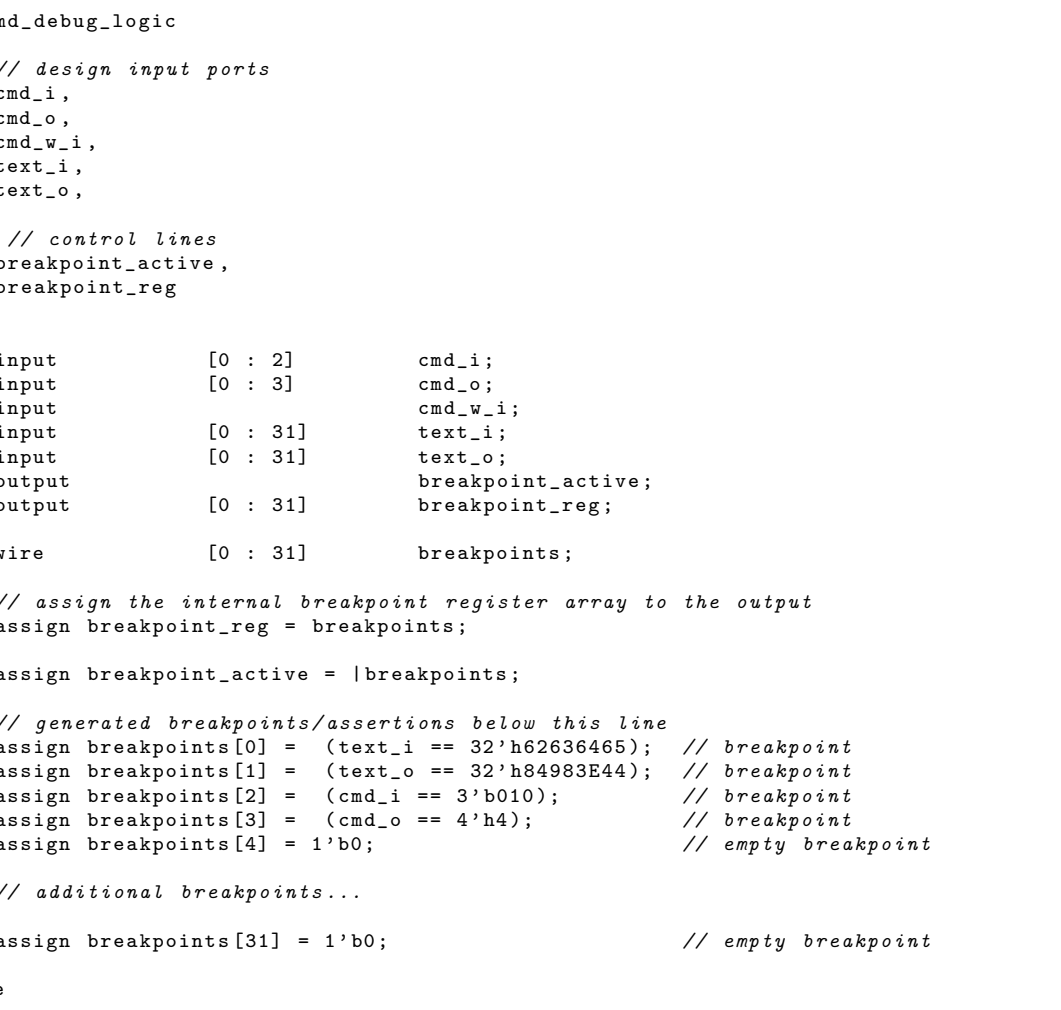

Listing 5.3: Generated Verilog breakpoint module for reconfigurable breakpoint region.

of the debug region. When implementing the debug region, overhead such as that with DRC is almost entirely eliminated.

\section{Stepping Logic}

Clock stepping is an another function of the PDC and utilizes a dedicated step counter and breakpoint logic as a separate control to the clock buffer. The PDC can be programmed through the API to step the design an arbitrary number of clock cycles with an accuracy of one to two clock cycles. This uncertainty is based on the operation of the clock buffer which transitions the clock on the following rising clock edge after the select line has been asserted [3]. Based on the timing of the triggering event, the actual transition may not occur until the following rising edge, resulting in a two cycle latency. The stepping function 
presented here is unique in that the design's actual clock is used rather than an artificial or synthesized clock, enabling stepping to be performed at the correct frequency and duty cycle. To the best of our knowledge, this is the only debug implementation that enables debug stepping at the design's target frequency using the system clock.

A memory-mapped register in the PDC maintains the target number of clock cycles to be stepped and is set through the API. The design is then set to execute by switching the PDC's clock buffer to the system clock. Once the counter expires, a suspend signal causes the clock buffer to disconnect the clock once more and hold the clock line high, allowing inspection of the design.

While a software-controlled clock would be easier to implement and enable a variety of additional functionality, it would not guarantee glitch-free operation. Treating a clock line as ordinary logic assumes the risk that the implementation tools would route clock signals through ordinary logic lines, a condition known as gated logic. The logic fabric and its routing are not designed to maintain clock signal integrity. Designs which utilize ordinary logic for clocking signals do so at their own risk since the tools will no longer be able to properly assess timing information.

\section{ICAP Interface for State Readback}

The ICAP is a user-accessible configuration port, allowing similar access as with JTAG to register state. However unlike JTAG, the ICAP allows random access to arbitrary locations of the device without the need to shift out the entire device state, enables internal access directly from design logic rather than an external development tool, and allows access to configuration data as well as design register state. The ICAP is most frequently used by designs that employ partial reconfiguration, whereby a reserved region is reconfigured directly from the design as the rest of the design continues normal, uninterrupted operation. Through the 
ICAP, a design can reconfigure itself as part of its operation, reconfiguring a region to perform different tasks, similar to memory overlays in software and thereby raising overall utilization of a smaller, less expensive device. Otherwise, larger devices are required to accommodate all possible logic configurations which might otherwise be left unused a majority of the time [36]. The ICAP is used to increase the visibility into a design since it has a full, unobstructed view of the entire device as a random memory.

The smallest granularity of an ICAP access is the configuration logic frame, a vertical stack of 1312 bits (or 41 32-bit words) that configure a column, while a horizontal series of columns form a row. Each column is a collection of various FPGA resources with a HCLK clock tile at the center, occupying the center 32-bit word of a configuration frame. The remaining 640 bits above and below the center word configure the resources of those regions respectively [42]. A diagram of a configuration frame is given in Figure 5.7. On generations through the Virtex-II, the configuration architecture varied within the family and different devices each had different configuration frame sizes making development and characterization difficult. Beginning with the Virtex-4 family, the configuration architecture became standardized across devices and continues to be consistent across families as described [40]. While the actual purpose of each bit within the configuration frame is proprietary, Xilinx tools have the option to produce a file that maps individual design bits to bitstream locations and configuration frame bit addresses. Such a mapping would enable the construction of a symbol table for FPGAs. The generation and parsing of such a file to produce this mapping is discussed in Section 5.2.3.

While both reading and writing to the ICAP are supported, it is not possible to directly modify a design's internal state through the ICAP. While both configuration and design state can be read through the ICAP, it is only possible to write configuration data (such as bitstreams when performing partial reconfiguration) that directly affect an entire configuration frame. Initial values for registers and memories are found in the configuration data, 
however these are transferred to the state bits during device initialization when the device's Global Set/Reset (GSR) signal is asserted. As the name implies, GSR is a device-global signal which causes all the bits in the configuration data frames to be shifted into the device's logic bits which function as bits of registers. While moving state in the opposite direction is a non-destructive (read-only) operation that occurs when the ICAP's CAPTURE command is asserted, a GSR operation destructively writes all state bits of the entire device, causing the device to lose its state. The MetaWire [71] project successfully reverse-engineered the process of selectively writing configuration bits to reprogram BRAMs. This allowed a Network on Chip (NoC) to be implemented using the preexisting configuration channels and did not require the significant resources needed for an actual NoC on an FPGA. However the method was highly device- and architecture-dependent and did not lend itself well to integration within a design. An illustration of the relation of configuration frame bits to device resources and the movement of data between the two is shown in Figure 5.7 [42].

\subsubsection{Unified Software Interface}

The debug component of DMD utilizes two tightly-coupled software platforms to provide the access and visibility of a software debugging environment to FPGAs. One is a highlevel user interface on a conventional workstation that runs both the vendor and the DMD tools allowing them to easily exchange data. The workstation application raises the abstraction of common low-level debugging tasks from an architectural-specific level to a symbolic level consistent with the original design. Additionally, traditional workstations can handle resource-intensive tasks such as building a model of the FPGA, physically mapping the design to this model, and providing an intuitive user interface similar to those found in software environments. The other software platform executes on a MicroBlaze processor on the FPGA alongside the design and provides a point-of-presence to control execution and perform state 

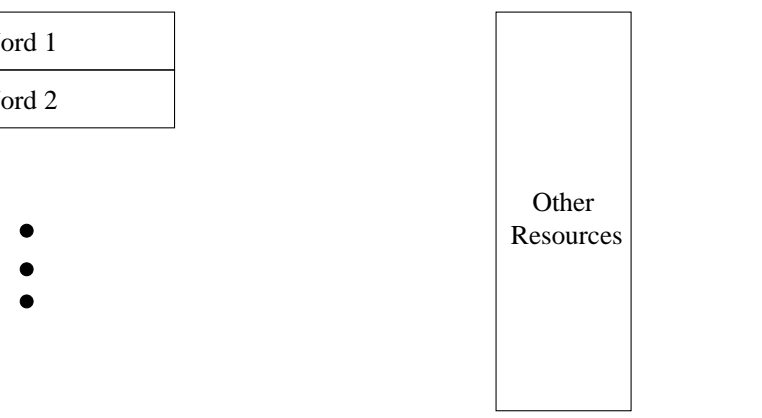

\begin{tabular}{|c|}
\hline Word 20 \\
\hline Word 21 \\
\hline Word 22 \\
\hline
\end{tabular}
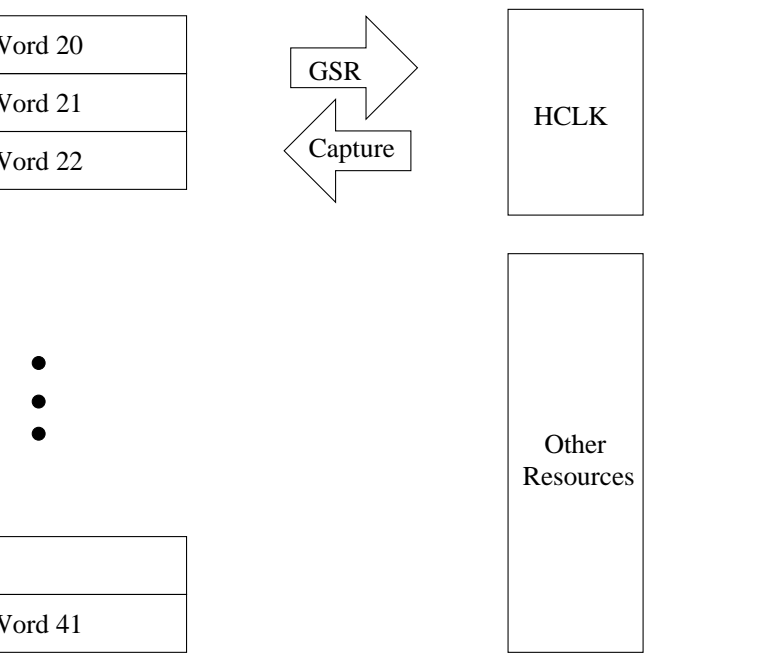

Configuration Frame/Bitstream

Figure 5.7: Relationship between device resources and configuration frames.

readback through the ICAP. The combined application's interface was modeled after the GNU gdb debugger [72] and provides an interface to FPGA design debugging familiar to software debuggers. Delegation of commands and processing is used in the combined platform to provide a single, unified interface. Commands are transparently processed either on the workstation, the FPGA-based processor, or reformatted on one in order to be processed on the other, and provide full visibility and control of a design from the single interface.

The limited resources of the onboard processor require its software to be primitive and therefore difficult, if not impossible, to manually manipulate. A compilation directive can either eliminate or include other interactive elements from the console application such as 
Table 5.4: Summary of on-chip debugger commands.

\begin{tabular}{|l|l|}
\hline Command & Description \\
\hline \hline help & $\begin{array}{l}\text { Prints a help message with information on } \\
\text { each available command }\end{array}$ \\
\hline Clears the console screen \\
\hline run & $\begin{array}{l}\text { Run the design directly connected to the sys- } \\
\text { tem clock }\end{array}$ \\
\hline step $[$ cycles] & $\begin{array}{l}\text { Step the design a specified number of clock } \\
\text { cycles, one clock cycle if none are specified }\end{array}$ \\
\hline stop & Stop the design from executing \\
\hline $\begin{array}{l}\text { print } \\
<\text { frame }><\text { offset0 }>[\text { offset1... }]\end{array}$ & $\begin{array}{l}\text { Retrieve and print the value or values found } \\
\text { at the given frame and one or more offsets. }\end{array}$ \\
\hline ping & $\begin{array}{l}\text { Respond with a message indicating that the } \\
\text { debug software is functional. }\end{array}$ \\
\hline status & $\begin{array}{l}\text { Provide general diagnostic information about } \\
\text { the PDC's internal registers. }\end{array}$ \\
\hline enable $<$ index $>$ & $\begin{array}{l}\text { Enable the breakpoint identified by the index } \\
\text { number. }\end{array}$ \\
\hline disable $<$ index $>$ & $\begin{array}{l}\text { Disable the breakpoint identified by the in- } \\
\text { dex number. }\end{array}$ \\
\hline info mask & $\begin{array}{l}\text { Print the breakpoint mask indicating which } \\
\text { breakpoints are enabled. }\end{array}$ \\
\hline info active & $\begin{array}{l}\text { Print the active breakpoint mask to show } \\
\text { which breakpoints are currently active. }\end{array}$ \\
\hline
\end{tabular}

command-prompts, user-friendly messages and formatting, and other non-essential elements that improve the usability. These changes turn the FPGA-based processor into a debug server that the workstation application communicates with using a special protocol. Commands are encoded, replacing design names with frame and offset locations or translating intuitive command names to the local processor's more abbreviated versions. The user workstation also reformats the FPGA-based processor's responses to a more more readable format to improve usability. Table 5.4 gives a summary of the commands available on the embedded processor. 
The two platforms are linked using a serial line. Communication between FPGAs and the outside world is a challenging tasks. FPGAs are targeted towards low-level, high-speed applications and can have hundreds of high-speed pins. High-level, interface connectors such as those for keyboards and monitors are available but are not standard on most development platforms. To establish a reliable, portable communication medium, a conventional serialconsole was implemented. Serial links have been used reliably in computers for decades and although they have been eclipsed many times by faster and more sophisticated communication mediums, they are still universally prevalent because of their simplicity. Although mostly invisible on modern computers and even microprocessors, serial access is possible on the smallest of devices, even such commodities such as USB memory drives. At least one serial interface is found on almost all FPGA development platforms, regardless of cost and inexpensive USB-to-serial converters are available for modern workstations where the serial connector has all but vanished.

Despite relatively slow performance (the fastest transfer rates are at $100 \mathrm{kbps}$ ), serial links continue to thrive because of protocol's simplicity and low-cost of implementation. Serial links do not require a complex processor, protocol, memory, or software stacks, as does USB or Ethernet. Synthesizing the required cores in reconfigurable logic for serial interfaces requires few resources and IP is readily and freely available from multiple sources. And unlike both Ethernet and USB, serial implementations are easily portable across hardware vendors. Serial interfaces were implemented and a simple communication protocol was developed to leverage these benefits so that this work was portable across development boards with minimum effort.

The workstation user application was written for the Linux platform in $\mathrm{C}++$ as a commandline application. Once the application is started, a command-prompt is presented. A connection to the FPGA processor is then established and validated over the serial line. Next, the 
device and logic models are built in order to provide a navigable model for the mapping of design elements to the physical resources of the FPGA. The GNU Readline [73] library was used to provide the familiar command-prompt found on most Unix/Linux applications and shells, including command-completion and command history. Table 5.5 gives a summary of the workstation application commands.

\subsubsection{Logic Model and Symbol Table Creation}

In order to successfully retrieve and assemble the individual bits from the configuration logic frames into register values, a symbol table is required. Logic allocation files are an optional output file produced during bitstream file generation and map individual bits of the named design registers to both their absolute location in a serial JTAG chain and the configuration logic frame and an offset within the frame. The logic allocation file also includes additional information, such as the physical device resource corresponding to each bit. A sample line of a logic allocation file is shown in Figure 5.8. By parsing the logic allocation file, a map can be generated defining where in the FPGA fabric each bit of a design register resides.

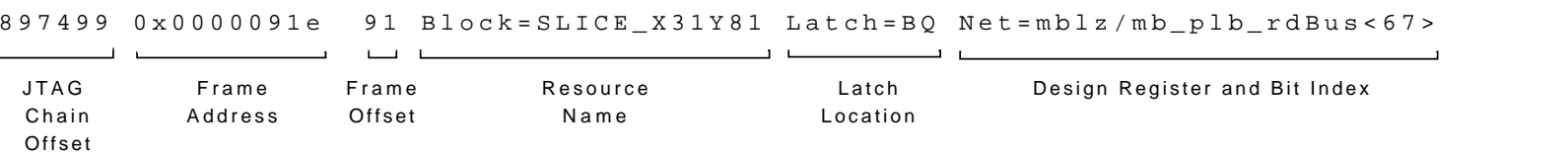

Figure 5.8: Logic allocation record format.

For a modestly-sized design, the logic allocation file can be tens of Megabytes. As there is no practical way to process such a large file on the FPGA itself, a conventional workstation is required. A parser (whose source can be found in the Appendix) was developed to read in the logic allocation file and generate a lookup table whereby hierarchical design names could be mapped to a list of configuration logic frame addresses and offsets. 
Logic allocation files are space-delimited, fixed-width column ASCII text files. Two types of records are found in logic allocation files: Info and Bit records. Info records define special meanings for configuration bits while Bit records exist for each design bit and maps it a physical location in the FPGA. There are very few Info records in a logic allocation file, and they are unnecessary for this application. In the logic allocation file, the record type (Bit or Info identifier) is found in the first column.

The second field of a Bit record is the absolute location of the bit in the JTAG chain. The third and fourth fields are the frame and offset values, respectively. The frame field identifies the configuration frame in which the bit resides, while the offset defines the offset beyond the initial NULL frame where the actual bit can be found. Special care is required when determining the offset once the frame has been retrieved since a NULL frame and single word are also returned at the beginning of the frame, which must be discarded. It is assumed that this initial NULL frame represents proprietary configuration data. Therefore, space sufficient for two frames plus one additional word must be allocated in the application, and all references must be offset by that amount when accessing data read from the ICAP. Additionally, bits are inverted from their actual value and must be inverted before being interpreted as a register value.

Next, the Block field indicates the unique name of the FPGA resource used. Design state registers are located in Slice or Input/Output Buffer (IOB) resources. However, design registers are rarely, if ever, allocated to IOBs. The Latch field following the Block identifies which latch within the block the bit occupies. Finally, the Net field identifies the design's hierarchical name, its index if the register is a vector, or both the bit's index and array reference in the case of multi-dimensional vectors, such as in memories.

The construction of the symbol table only requires the use of Bit records and Net fields, easily found using a token-based parser. A Ram record maps out the allocation of generalpurpose logic implemented as a ROM or RAM. The parser begins by discarding all comments, 


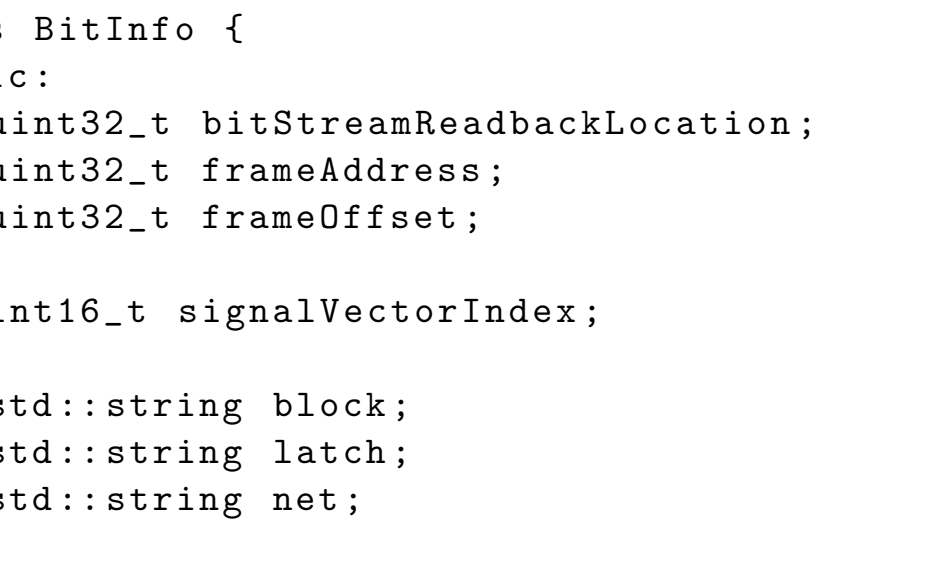

Listing 5.4: BitInfo data structure.

delimited by a "\%" and Info lines. Bit lines mapping blocks with Slice resources are read, while RAM and ROM records are discarded.

The BitInfo data structure, shown in Listing 5.4, represents a single bit when mapped from the logic allocation file to a location in the FPGA. Information contained in this structure includes the absolute location of the bit if shifted out of the device, the frame and offset addresses when accessed via the ICAP, as well as the index of the signal if it appears in a signal vector, such as a bus. Additional information includes the block and latch where the bit is located. Consecutive bits for vectored signals are stored sequentially in a list array in a BitInfo data structure; the array is then mapped to the design register name in a lookup table.

The lookup table is implemented as a map, keyed by the signal name since the full hierarchical name is globally unique in the design. This means that there is no risk of collisions, which occur when duplicate keys are generated. Collisions can be managed either by detecting them programmatically before inserting a new value and implementing the map's value storage container as a list or through the use of a multimap which allow duplicate key values by 
design. Map complexity is logarithmic with the size of the map, denoted by $O(\log n)$. The search for keys is logarithmic as the keys are stored in a binary tree.

Once the entire logic allocation file is processed, the information is available to the console workstation application. The ample memory and disk resources of a conventional workstation make user interaction easier to enhance. The GNU Readline library is a standardized tool for creating user-friendly command-line prompts and is found in many open-source projects, including Linux shells such as bash and csh. Readline provides command-line editing capabilities, such as history and tab-completion to applications, making commandline applications more intuitive and easier to use. Using the tab-complete capabilities of Readline, the design can be explored from a command-line using the names extracted from the logic allocation file. Users can press the TAB key to list all available design names, or enter a few characters of a name and view those that match without knowing or having to memorize the design's hierarchy. The design's hierarchy can be navigated and its state inspected.

The print function of the user console application uses the logic model built from the logic allocation file to assemble a series of print commands targeted towards the embedded processor. Where possible, consecutive bits found in the same frame are combined into a single print command to accelerate processing. When entered, the register name is searched for in the register name table of the logic model, and the corresponding list of BitInfo structures is retrieved. The configuration frame of the first bit is read and stored, and consecutive bits are checked for the same configuration frame. Reducing the number of round-trips to the FPGA is key to reducing processing overhead, and consecutive bits in a register have a high likelihood of existing in the same or neighboring frames. As the bits are processed, the offsets are stored in a list until a non-matching frame is encountered. The set of bits existing in the same frame are sent to the embedded processor, which then retrieves 
the bit values, inverting them before serially shifting them into a register and returning the result. The retrieved values are shifted into a register and then displayed once all the bits have been collected. The algorithm for retrieving register values is given in Figure 5.9.

The list of BitInfo structures is sorted on its first access which prevents lists that are never accessed from being unnecessarily sorted. The sort algorithm is implemented as a bubble sort with complexity $O(n \log n)$. Bubble sorts are the simplest to implement, but are not the most efficient. In a bubble sort, two adjacent elements are compared and swapped if they are out of order before advancing to the next pair of elements. The list must be iteratively traversed until all the elements are in order which is when the list can be traversed without performing any swaps. Bubble sorts are not as time efficient as other sort algorithms, but are the most space efficient. Bubble sort requires only $n+1$ memory locations for sorting a list of length $n$ since only one additional location is needed for temporary storage during the swap. Bubble sort is sufficient for this application since the longest list to be sorted will be of length 32 .

The calling program passes the name of the target register to the procedure which first initializes variables for the resultant register value and intermediate fragments, variables for determining when the configuration frame changes, and lists for the locations of bits and offsets (lines 2-7). A list of bit locations is returned from the table which maps signal names to physical locations as seen on line 8 . To prime the first round of processing, the first location in the returned list of bit locations is decomposed to a physical frame address and offset. This frame offset is the first offset to be appended to a list of frame offsets (lines 9-11). Consecutive bits have a high likelihood of being physically located close to one another, therefore a simple optimization is to group all the bits located in the same frame into a single frame access. A variable storing the previous frame is written with the current frame's address enabling the loop to distinguish when the frame address changes (line 12). 
1: input : A text string identifying a SignalName

2: output: The value of the register denoted by SignalName

3: registerValue $=0$;

4: previousFrame $=0$;

5: currentFrame $=0$;

6: frameOffsetList $=[]$;

7: bitLocations $=[]$;

8: registerFragment $=0$;

/* Retrieve the mapping structures for the given signal

9: bitLocations = lookupMappingInfo(SignalName);

/* The first bit must be handled outside the loop since successive frames will be compared

10: bit $=$ pop(bitLocations);

11: $\{$ frameAddress, frameOffset $\}=$ getFrameInfo(bit);

12: append (frameOffsetList, frameOffset);

13: previousFrame $=$ currentFrame;

/* Handle the remaining bits in order

14: for bit $\in$ bitLocations do

15: $\quad\{$ frameAddress, frameOffset $\}=$ getFrameInfo(bit);

/* if the frame changes, send the command to FPGA software */

16: $\quad$ if currentFrame $\neq$ previousFrame then

17: $\quad$ registerFragment $=$ getFrame(previousFrame, frameOffsetList);

18: $\quad$ registerValue $=($ registerValue $\ll$ size $($ frameList $)) \vee$ registerFragment;

19: clear (frameList);

20: $\quad$ append (frameList, frameOffset);

21: previousFrame = currentFrame;

22: registerFragment $=$ getFrame (previousFrame, frameOffsetList);

23: registerValue $=($ registerValue $\ll \operatorname{size}($ frameList $)) \vee$ registerFragment;

24: return registerValue;

Figure 5.9: Algorithm to retrieve register values.

The remaining bits are processed in a loop which first checks if the current frame is different from the previous (line 15). If this is the case, the frame address and the list of the frame offsets is sent to the processor on the FPGA which replies with a fragment of the requested values (line 16). The returned fragment is OR'ed with the running result which must first be shifted left the width of the fragment size (line 17). If the current frame is the same as the previous frame, the offset is appended to the list of offsets and the loop is repeated (lines 19-20). Once all the bit locations have been processed, one final iteration is required to 
process the remaining bits into the result register which is returned to the calling application (lines 21-23).

When printing values, the FPGA-resident processor first checks that the design is not running. A running design will cause inaccurate values to be printed. Then, a capture command is issued to the ICAP to transfer register values to the configuration frame bit locations. The selected configuration frame is read from the ICAP, first discarding the NULL frame and NULL word which precede the requested frame. The integral part of the offset divided by 32 gives the index of the target word, while the offset modulo 32 gives the correct bit of the target word. These equations are presented in Equations 5.1 and 5.2.

$$
\begin{gathered}
\text { WordIndex }=\operatorname{int}\left(\frac{\text { offset }}{32}\right)+\text { NullFrameOffset } \\
\text { BitIndex }=\text { offset } \bmod 32
\end{gathered}
$$

\section{$5.3 \quad$ Summary}

This chapter presented the implementation of two development and debug methodologies. Both employed an embedded processor and a custom peripheral to provide a general-purpose interface to the module or design to be tested. HLV's implementation requires considerable memory resources as it is a capture methodology, first executing the original high-level reference model, storing the results, then staging the same input data for the hardware execution. HLV's peripheral contains FIFOs for both input and output. The input data is first stored in the input peripherals, then released to the inputs of the hardware device which is continuously connected to the system clock. This implementation is unique in that the hardware implementation is compared to a high-level reference model without significantly 
altering either, and the comparison is done at the target frequency. The processor serves as a platform for software-based hardware testbenches.

LLD likewise uses a microprocessor and the PDC as its custom peripheral, but for different purposes. In this case, the processor is used as a debug server on the FPGA and as a pointof-presence in what would ordinarily be an inaccessible location. A workstation application is then the client, constructing series of commands that are sent to the FPGA processor and reformatting the results. The workstation application is needed for hosting the large models of the FPGA and the design which cannot be processed on the FPGA-side processor. The two applications present a unified interface, delegating commands to the appropriate platform as needed. The PDC hosts clock control logic for error-free transitions to stop the design, controlled by a rapidly reconfigurable breakpoint module, a user-programmable step counter, or the command console. Additionally, the PDC also hosts the logic required for reading all the design's registers, although this functionality is largely controlled from both the software interfaces. 
Table 5.5: Summary of workstation commands.

\begin{tabular}{|l|l|}
\hline Command & Description \\
\hline \hline run & Begin execution of the design when stopped \\
\hline stop & Halt execution of the design when running \\
\hline step [steps=1] & Step the design steps clock cycles (default one) \\
\hline source $<$ filename $>$ & $\begin{array}{l}\text { Read the commands listed in the file filename and } \\
\text { execute them as if they were entered at the com- } \\
\text { mand prompt }\end{array}$ \\
\hline connect $<$ port $>$ & Connect to the serial device given in port \\
\hline disconnect & Disconnect from the serial device \\
\hline continue & Continue execution \\
\hline constrain $<$ condition $>$ & Add the constraint/assertion given by condition \\
\hline break $<$ condition $>$ & Add the breakpoint given in condition \\
\hline delete $<$ index $>$ & $\begin{array}{l}\text { Delete the breakpoint given by index from the } \\
\text { breakpoint table }\end{array}$ \\
\hline info breakpoints & $\begin{array}{l}\text { Print the active breakpoint table, the status of the } \\
\text { breakpoint masks and enables }\end{array}$ \\
\hline disable $<$ index $>$ & $\begin{array}{l}\text { Disable the breakpoint given by index by disabling } \\
\text { its corresponding bit in the breakpoint mask }\end{array}$ \\
\hline enable $<$ index $>$ & $\begin{array}{l}\text { Enable the breakpoint given by index by disabling } \\
\text { its corresponding bit in the breakpoint mask }\end{array}$ \\
\hline print $<$ index $>$ & Print the value of the given register \\
\hline loadlogic $<$ file $>$ & $\begin{array}{l}\text { Load the logic-allocation file and use its contents } \\
\text { as the logic model for the current design }\end{array}$ \\
\hline addport $<$ name $><$ size $>$ & $\begin{array}{l}\text { Add a port with the given name and size to the } \\
\text { breakpoint region for use as an input }\end{array}$ \\
\hline delport $<$ name $>$ & $\begin{array}{l}\text { Delete the port as a an interface port for the break- } \\
\text { point region }\end{array}$ \\
\hline Clear the console screen \\
\hline ping & Ping the remote device \\
\hline help & $\begin{array}{l}\text { Print a help message with information about all } \\
\text { the commands }\end{array}$ \\
\hline quit & $\begin{array}{l}\text { Disconnect the remote device and exit the appli- } \\
\text { cation }\end{array}$ \\
\hline
\end{tabular}




\section{Chapter 6}

\section{Evaluation}

Chapter 5 presented the implementation of both the HLV and LLD solutions that bridge the model verification gap and increase the visibility, agility, and controllability of implemented FPGA designs. In Chapter 6, both approaches are evaluated and the results compared against similar products.

To evaluate these approaches, benchmark designs were developed and validated using the HLV and LLD tools. All designs targeted a Xilinx XC5VLX110T-1 F1136 FPGA using the Linux Xilinx ISE 12.4 design suite with the PR patch running on a $2.80 \mathrm{GHz}$ Intel Core i7-930 processor with 24 GB of RAM. Both HLV and LLD employ a Xilinx MicroBlaze processor with $64 \mathrm{kB}$ of on-chip BRAM and built with a 115,200 Baud serial console interface for communication.

\subsection{High-Level Validation}

HLV links the high-level reference model to an implemented design on an FPGA. The design is tied to the processor hosting the testbench software through a custom peripheral that links it to the DUT. The benchmark design was a SHA-1 message digest, a common cryptography core in widespread use. 


\subsubsection{Secure Hash Algorithm}

The Secure Hash Algorithm (SHA-1) is a widely used iterated cryptographic hash algorithm used to produce a 160-bit message digest from a input message block. The input message must be padded to produce a 512-bit message block. Hashes are used to uniquely fingerprint a block of data, such as a message or application, to detect if the data has been altered or corrupted and are commonly known as checksums. SHA-1 operates by rotating five 32-bit word segments of the data while applying bit shifts and predefined constants to the original message. Eighty rounds of these rotations or compressions are repetitively applied. A hash is considered unique for each message, with the probability of finding a message to produce an identical hash computationally infeasible. While vulnerabilities have been found, SHA-1 continues to be widely implemented [74]. As a useful function in both hardware and software applications, SHA-1 cores and software libraries are widely available. Many of the operations can be performed in parallel, lending itself well to a hardware-based implementation. A block diagram of the SHA-1 algorithm is shown in Figure 6.1 [75]. Each of the five input blocks is a 32-bit word which is shifted right, with the fifth word being shifted around to the first position. While some of the inputs are not modified at all, others such as the second and fifth inputs (B and E) are shifted or transformed. The function $f$ and constants $K_{n}$ and $W_{n}$ are determined by the round number. An excellent discussion of the SHA-1 algorithm can be found in [74].

\subsubsection{Results}

Evaluation for HLV was performed on a SHA-1 core obtained from the OpenCores archives [76], a repository of open-source hardware IP. A high-level reference model of the SHA-1 al-

gorithm was obtained from a different source. After extracting the control signal interaction 


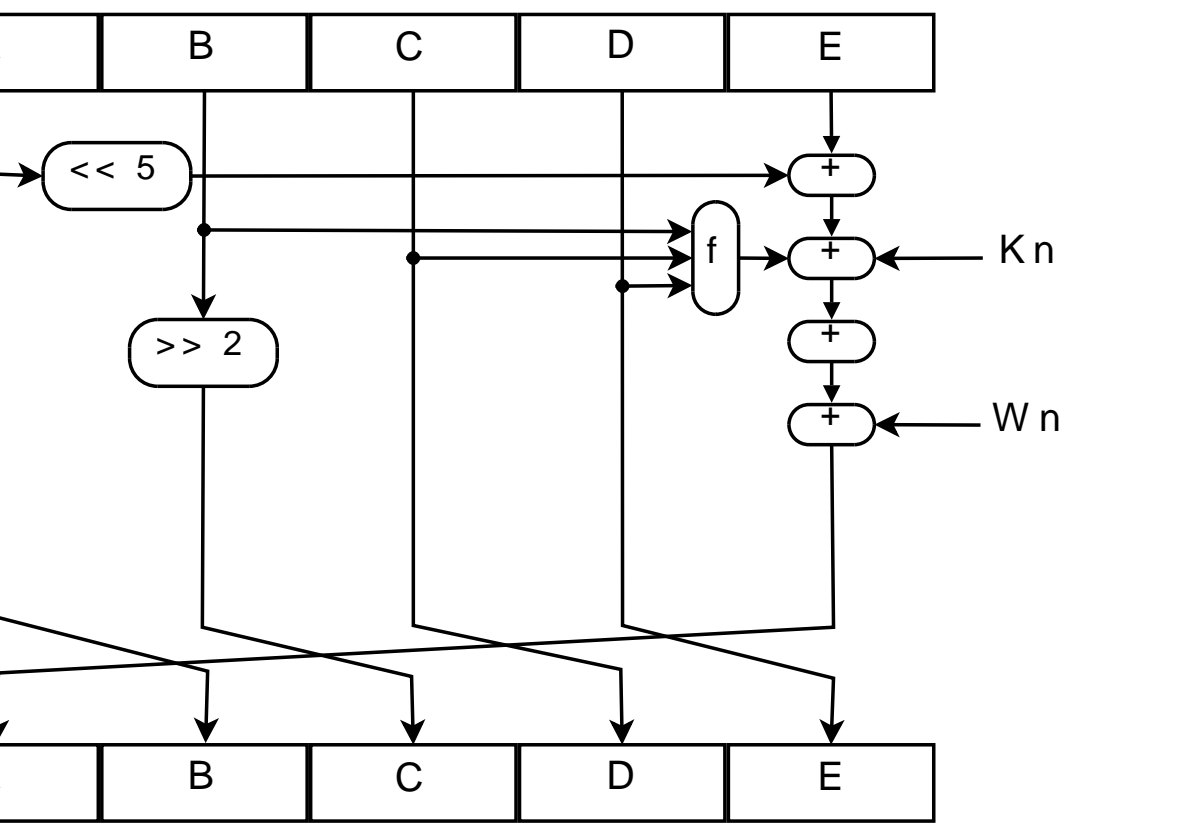

Figure 6.1: SHA-1 block diagram.

from a simulation of the hardware core, the software testbench was programmed. Programming of the testbench took approximately 20 minutes, including developing an appropriate hardware reset routine and entering the correct sequence for data and control. The simulation data provided the basis for correctly programming the framework to reproduce the correct interaction of control signals and specify the output window for results. However, once this information is incorporated into the testbench, any changes that affect latencies or final results will be detected. The captured hardware results were used to validate the hardware implementation against the simulation. It is possible to programatically expand and move the capture window's limits, enabling scanning of control or data output to detect other expected results.

The MicroBlaze processor was configured for $125 \mathrm{MHz}$ operation with $64 \mathrm{kB}$ of RAM for data and instruction memories, and a 115,200 Baud serial interface. The FSL input queues were configured with 256-input words while the capture queues had 32 words. The FSL queues were implemented using distributed logic to save BRAM resources for the processor or the 


\begin{tabular}{|c|c|c|c|}
\hline \multicolumn{2}{|c|}{$\begin{array}{l}\text { Executing software } \\
\text { Queuing input data } \\
\text { Capture window set } \\
\text { Executing hardware }\end{array}$} & \multicolumn{2}{|c|}{$\begin{array}{l}\text { model...done. } \\
\text { for hardware model...done. }\end{array}$} \\
\hline Cycle & Output & Expected & \\
\hline 175 & $84983 E 44$ & [84983E44] & {$[\mathrm{OK}]$} \\
\hline 176 & $1 \mathrm{C} 3 \mathrm{BD} 26 \mathrm{E}$ & [1 C3BD 26E] & {$[\mathrm{OK}]$} \\
\hline 177 & $\mathrm{BAAE} 4 \mathrm{AA} 1$ & {$[\mathrm{BAAE} 4 \mathrm{AA} 1]$} & {$[\mathrm{OK}]$} \\
\hline 178 & F95129E5 & [F95129E5] & {$[\mathrm{OK}]$} \\
\hline 179 & E54670F 1 & [E54670F 1] & {$[\mathrm{OK}]$} \\
\hline
\end{tabular}

Listing 6.1: Transcript of an HLV test session.

design itself. The HLV testbench occupied only $14 \%$ of the Slice resources and $20 \%$ of the BRAMs. The entire application required only $13.6 \mathrm{kB}$ of the total $64 \mathrm{kB}$ BRAM available, allowing for larger applications. The time required to rebuild the software testbench and reintegrate with the bitstream is under 30 seconds, with compilation taking 13 seconds, and the memory integration into the bitstream taking 14 seconds.

A transcript of an HLV test session is shown in Listing 6.1 demonstrating the execution of the software reference model, the configuration of the hardware, and finally the comparison confirming the match.

First, the software model of the SHA-1 is executed on the embedded processor, and the results stored. HLV does not include any standard means of collecting the reference model results as this is left to the testbench developer depending on the requirements. Then, the data are loaded into the input queues of the DUT exactly as during simulation. Once the input data is queued, the capture window is set as a function of the number of clock cycles from the beginning of execution. The input queues are then released and a counter triggers the capture of the output once it is in the range of the capture window. The data is then compared to output of the reference model. Figure 5.1 outlines HLV's data flow and results comparison, while Figure 5.3 illustrates the capture window mechanism. 
Research at the University of Florida (UF) $[66,77]$ investigated an approach to validating designs implemented with high-level synthesis and appears to be the only work devoted to in-circuit validation against a high-level language specification. We were unable to identify any other product - research or commercial - that compared a high-level specification directly with implemented hardware. While the UF approach relied entirely on HLS, assertions written in the native design entry language were incorporated into the application. HLS was discussed in Section 2.4. Designs implemented with HLS are typically validated in simulation and prove difficult to validate or debug once committed to hardware. This research implemented the ANSI-C assertions as asynchronous hardware logic in the design and requires a communication medium between the design and the developer platform. Assertions written into the source code are synthesized into the design as asynchronous logic that activates once the assertion becomes true.

The UF research's target objectives are much different than that of HLV. The UF project is not intended to address quick turnaround or even bridge the model verification gap as the design entry medium is already a high-level language. Rather, the UF project aims to boost developer confidence in the machine-generated RTL to catch translation errors and transparently integrate with complex designs without affecting timing closure. For instance, one concern is the overhead required to implement assertions in for loops. Unrolling the for loops would create multiple instantiations of the same assertion logic which in turn might affect timing.

\subsubsection{Summary and Future Work}

HLV is a unique approach to implementing software-based hardware validation. Designs were validated at target frequencies of $125 \mathrm{MHz}$ without the need to control the system clock or halt the design. No other projects have been identified that bridge the model verification gap in this manner. 
While not necessary for its operation, improvements could be made to HLV's software. Manually programing testbenches is laborious, requiring effort in transliterating simulation results into the HLV testbench API. Most simulators either produce an open format such as Value Change Dump (VCD) file format or have API access to the simulation engine. Automated testbench generation would greatly improve the experience and usability of HLV. Additionally, HLV lacks hardware support for reacting to design signal interactions. Instead, validation currently depends on all interactions occurring after fixed latencies. Software interaction would require additional logic to halt the application while the queues are reloaded. A more robust approach would be to enable software programmable data generators that reacted to control signals, further reducing the required capacity for the input queues, and thereby enabling more elaborate and less-scripted testbenches.

\subsection{Low-Level Debug}

LLD aims to raise the visibility, controllability, and agility of low-level FPGA debugging. The effectiveness of these objectives are measured through the time required to accomplish routine tasks that are normally cost- and time-prohibitive. As previously defined in Section 4.2, visibility is the ability to view arbitrary state of the design, controllability is the ability to define parameters to halt and control the design with fine-level granularity, and agility is the ease at which these changes can be made to the debugging profile. The effectiveness of LLD is evident in large or high-utilization designs where implementation times can be long, which in turn limits the number of turns-per-day. Three large benchmark designs were created to evaluate LLD's effectiveness in improving visibility, controllability, and agility.

\subsubsection{Benchmark Designs}

To evaluate LLD, three designs with device utilizations of more $90 \%$ of the FPGA's configurable logic resources were created. The designs were implemented to fully meet timing 
(which is not the default objective of the tools), which extends the implementation times. To meet timing, PAR must iteratively place a component, attempt to route nets connected to that component, and evaluate the path based on the timing model for the device and the design's constraints. Timing analysis is complicated by the complex routing architectures and various iterative strategies such as logic duplication that consume more resources, further congest routing, but may make timing easier to achieve. The benchmark designs include the high-performance, server-class OpenSPARC, a mesh of Xilinx MicroBlaze soft-core processors interconnected by FSL links, and a network of floating-point operators generated by the FloPoCo compiler.

\section{OpenSPARC T1 Processor}

The OpenSPARC T1 processor [78] is an open-source implementation of Sun Microsystem's (now Oracle) high-performance server processor architecture based on the UltraSPARC T1 processor. The OpenSPARC is a 64-bit, 32-thread, fully pipelined processor. Unlike most soft-processors, the OpenSPARC has a virtual memory management unit capable of running a full, standard Linux distribution. Most soft-processors lack the complex logic required for virtual memory and are therefore limited to running real-time OSes which typically target small, embedded platforms. The OpenSPARC includes an embedded Xilinx MicroBlaze to handle network and other auxiliary functions. The OpenSPARC utilizes $99 \%$ of the evaluation platform's Virtex-5 LX110T's Slice resources, $17 \%$ of the DSPs, and $15 \%$ of BRAMs.

\section{FloPoCo Floating-Point Compiler}

Floating-point cores are cumbersome to implement in FPGAs due to their complexity and space requirements. Floating-point operations require multiple pipeline stages for even the 
simplest operations such as addition, which takes approximately six cycles to the most complex, such as a square-root operator which can take more than 20 cycles. No generalized, standard library exists and no FPGA vendors currently implement physical floating-point cores on their devices. The FloPoCo [79] project provides a tool that creates custom, parameterizable floating-point core targeted towards a specific device, leveraging architecturespecific resources and allowing the width of the mantissa and exponent to be defined as needed. Custom operators can be tuned for the target application and device and provide control over the amount of resources consumed, rather than creating operators compliant to standards such as the IEEE 754 Floating-Point Standard, which has stringent precision requirements. The benchmark application was generated with FloPoCo 2.1.0 and occupied $98 \%$ of the Slice resources with a target frequency of $250 \mathrm{MHz}$. All operators were generated with 8-bit wide exponents and 23-bit wide mantissas.

\section{MicroBlaze Mesh}

The final benchmark application developed was a cluster of Xilinx MicroBlaze soft-core processors, such as those used throughout this research. The 10-unit FSL-interconnected cluster included one master unit with a serial UART console for user I/O and nine coprocessor units. The MicroBlaze cluster consumed $92 \%$ of the target device's Slice resources and targeted an aggressive $200 \mathrm{MHz}$ clock frequency.

\subsubsection{Results}

LLD was evaluated on the three benchmark designs by comparing the time required to implement the base design, embed Xilinx ChipScope cores (the vendor's ELA solution), and to implement the same or comparable tasks using the LLD approach. For each of the designs, real time was recorded for the implementation. Since turns-per-day are a general 
metric used to measure development efficiency, real time is preferred over CPU time. CPU time is often used in conjunction with real time to determine how much time the processor was idle, presumably waiting on I/O or memory accesses. Synthesis times for subsequent builds are typically shorter since this stage is the only one in which each individual module can be independently built.

Typical debug scenarios include altering the perspective of the debug framework when different sets of signals need to be analyzed. With ELA frameworks, altering the monitored signals occurs after synthesis by inserting ELA cores into the netlist and then re-implementing the design. In contrast, LLD allows any arbitrary signal to be inspected without the need to re-implement. Furthermore, ELA frameworks embed the conditions that trigger signal capture as part of the overall design logic while LLD separates breakpoint logic from the rest of the design through the use of partial reconfiguration. While LLD does require a full re-implementation, it is limited to a small fraction of the entire design and the overhead of DRC, which takes proportionally longer with increasing design size, is eliminated by creating a separate, smaller design just for breakpoint logic. This results in a debug framework that requires a constant time to implement regardless of the overall design size or complexity.

\section{Benchmark Designs}

Base design implementation times are given in Table 6.1. For each design, timing closure was required which lengthened place-and-route times. As discussed in Section 2.2.1, PAR is responsible for routing wires through the FPGA's complex, programmable routing architecture while attempting to find routes that meet the timing constraints. All the other implementation stages were described in detail in Section 2.2.1. For the OpenSPARC, a complete build took over 3 hours, with initial synthesis accounting for approximately 55 minutes and PAR requiring nearly two hours of the implementation time. The FloPoCo design required over 
Table 6.1: Benchmark design implementation times (hours:minutes:seconds).

\begin{tabular}{|l|l|l|l|l|l|l|}
\hline \multirow{2}{*}{ Stage } & \multicolumn{2}{|c|}{ OpenSPARC } & \multicolumn{2}{c|}{ FloPoCo } & \multicolumn{2}{c|}{ 10 MicroBlaze } \\
\cline { 2 - 7 } & Base & ChipScope & Base & ChipScope & Base & ChipScope \\
\hline Synthesis & $0: 56: 23$ & - & $0: 05: 56$ & - & $0: 02: 04$ & - \\
\hline Build & $0: 01: 24$ & $0: 01: 19$ & $0: 00: 39$ & $0: 04: 30$ & $0: 00: 51$ & $0: 04: 30$ \\
\hline Map & $0: 23: 57$ & $0: 23: 15$ & $1: 37: 00$ & $3: 17: 00$ & $0: 19: 00$ & $0: 18: 36$ \\
\hline PAR & $1: 48: 43$ & $0: 30: 00$ & $8: 45: 00$ & $2: 56: 00$ & $8: 19: 00$ & $9: 36: 00$ \\
\hline Bitgen & $0: 05: 59$ & $0: 05: 07$ & $0: 02: 44$ & $0: 02: 58$ & $0: 03: 02$ & $0: 03: 14$ \\
\hline \hline Total & $3: 15: 29$ & $0: 59: 41$ & $10: 31: 19$ & $6: 20: 28$ & $8: 43: 57$ & $10: 02: 20$ \\
\hline
\end{tabular}

10 hours to implement, with nearly nine hours spent in place-and-route. The MicroBlaze cluster took over nine hours of runtime, with eight hours devoted to place-and-route.

Table 6.1 also shows the implementation times for inserting the Xilinx ChipScope Pro ELA tool into the benchmark designs. These times were often unpredictable, sometimes taking less time to implement than the base design itself, while at other times longer which is to be expected given the additional overhead of new resources and additional routing. Each case was run multiple times and consistently gave similar results. ChipScope insertion occurs on a synthesized netlist, therefore no time is reported for synthesis, while the rest of the flow remains unchanged. In the OpenSPARC design, the durations of all the stages were consistent with the exception of PAR which showed a significant and unexplainable speed-up. Some implementation flows incorporate previous builds as a guide for future ones, such as Xilinx's SmartGuide. This can significantly improve implementation times for design iterations where very small changes occur. However in this case, SmartGuide was not responsible for these speedups, nor is guided implementation available for all designs. Similarly, the FloPoCo design also experienced an overall decrease in implementation time, with the Map process taking twice as long as the base implementation and the PAR stage taking less. This is not uncommon with more recent versions of the tools which speculatively perform placement during mapping when the mapping can be iterated to prevent PAR from failing. It was 
noted that for this design, the placement was indeed performed during the mapping, however the overall decrease in execution time remains unaccounted for as no guided implementation was used. In the MicroBlaze benchmark, the overall execution time increased. In all cases, a complex design that takes hours to implement will also take hours to insert ChipScope. The observed decrease in execution time is not guaranteed to occur in all situations and as noted earlier, the presence or removal of ChipScope can unexpectedly alter the design introducing new timing challenges. Even with the reduction of implementation times for modifications to the ChipScope-enabled design, the overall time still presents a significant obstacle to increasing the number of turns-per-day. The benchmark design results are shown in Figure 6.2.

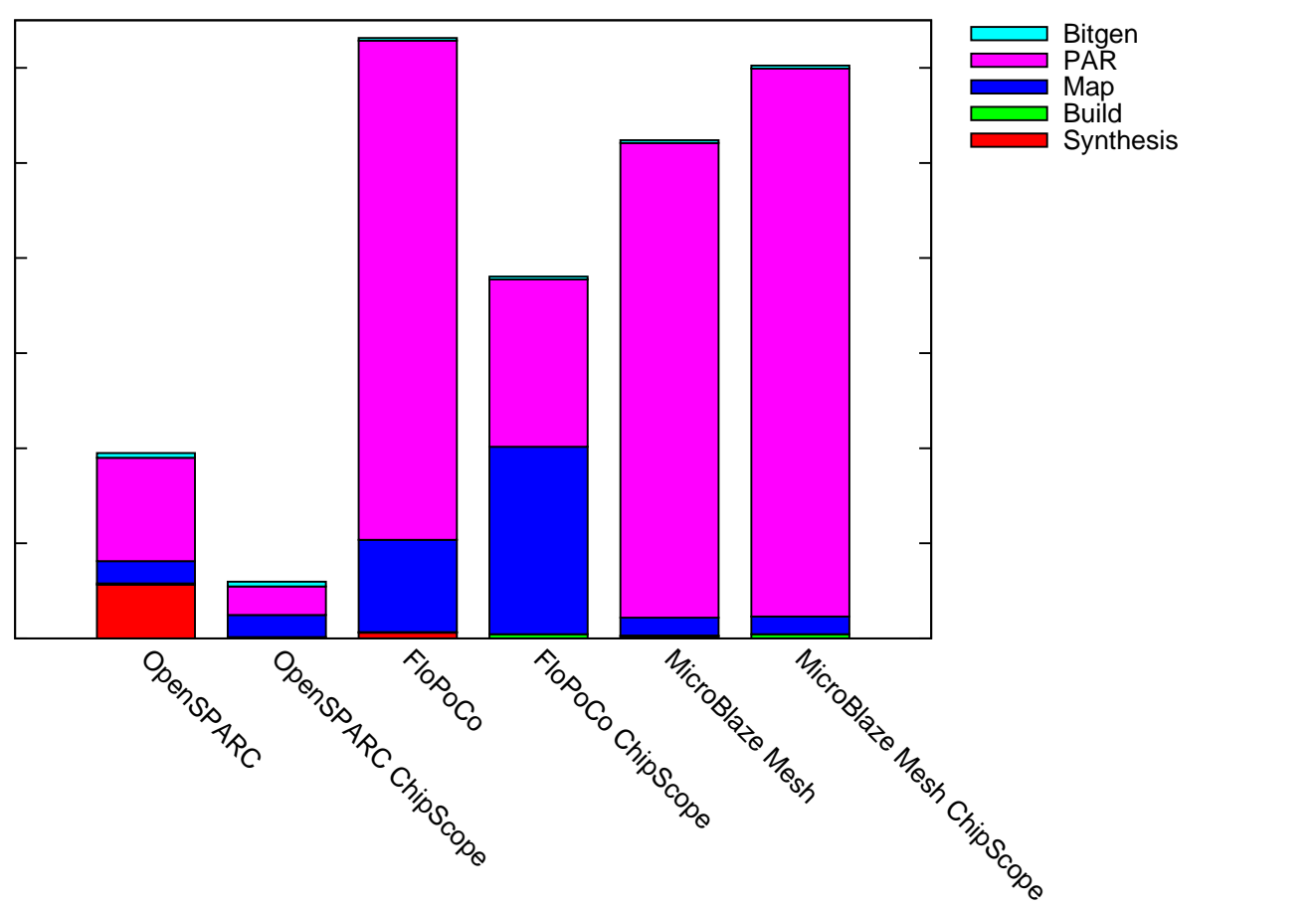

Figure 6.2: Benchmark design implementation times. 
Table 6.2: LLD implementation times (minutes:seconds).

\begin{tabular}{|l|l|}
\hline Stage & Real time \\
\hline Synthesis & $0: 08$ \\
\hline Build & $0: 04$ \\
\hline Map & $0: 39$ \\
\hline PAR & $0: 45$ \\
\hline Bit & $1: 35$ \\
\hline \hline Total & $3: 11$ \\
\hline
\end{tabular}

\section{LLD}

The LLD framework is isolated from the actual design by a partially reconfigurable region that partitions the debug logic into a region that can be implemented quickly. In comparison to ChipScope, similar tasks take anywhere from a few seconds to a few minutes as opposed to several hours. This speed-up is achieved through the "inverted bitstream" scenario outlined in Section 5.2.1. This technique eliminates the processing overhead of partially reconfigurable designs in which the entire design and not just the reconfigurable region are processed. As a result, LLD implementation time is constant regardless of the number changes or the overall design size. For the benchmark designs, the total implementation time of the debugging region took slightly over three minutes until the changes were ready to be used.

Table 6.2 gives LLD implementation times for a breakpoint region comprised of 204 Slices. The time required for synthesis and build were both under 10 seconds and consist of asynchronous logic that makes up the conditional breakpoints and assertions. Map and PAR each both take around 40 seconds each, considerably less time than the corresponding phases required for re-implementation when inserting or altering a ChipScope core. The minute and a half time required for bitstream generation is mostly due to DRC. While DRC can be eliminated which will noticeably reduce implementation time, its primary purpose is to detect configurations that might damage the device, making it a worthwhile investment. Figure 6.3 
shows a pie chart of data given in Table 6.2 showing the percentage of time spent at each phase of the implementation process. Unlike large designs, PAR now consumes approximately one-quarter of the execution time.

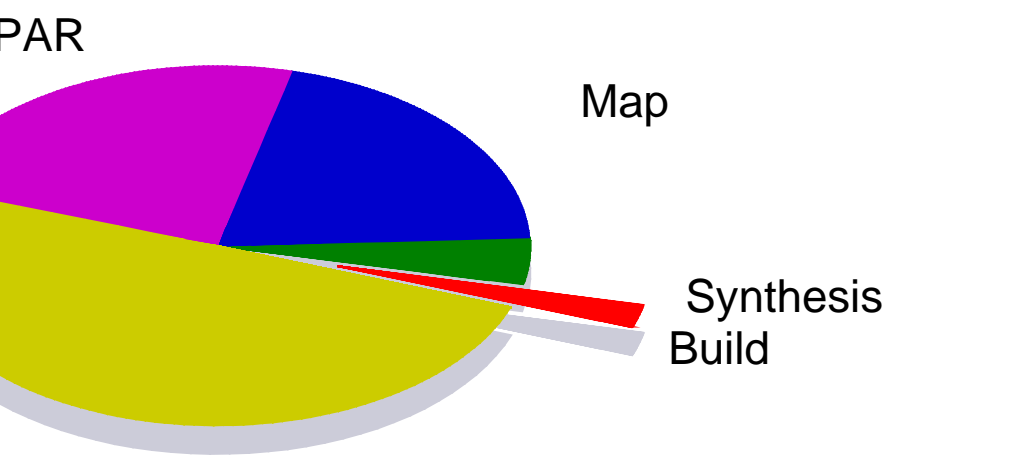

Bit

Figure 6.3: Percentage of time required for LLD implementation.

The number of resources required for a breakpoint region varies depending on the number of inputs, breakpoints, and the complexity of the logic implemented. However, this region requires only Slices which does not noticeably compete with the target design as would other resources. Even with high-utilization designs such as those implemented as benchmarks, both Map and PAR can be configured to expend extra effort and attempt to more tightly pack logic. In contrast, ELAs require a significant number of BRAM resources to record signal activity. This requirement increases proportionally with the number of signals to be monitored or the number of samples to be recorded. In one experiment, a simple set of breakpoints occupied as few as 10 Slices. Oversizing the breakpoint region can avert contention should the number or complexity of the breakpoints outsize the current region. However should this happen, the PATIS framework is capable of resizing and adjusting the floorplan as needed, likely with no additional time. 


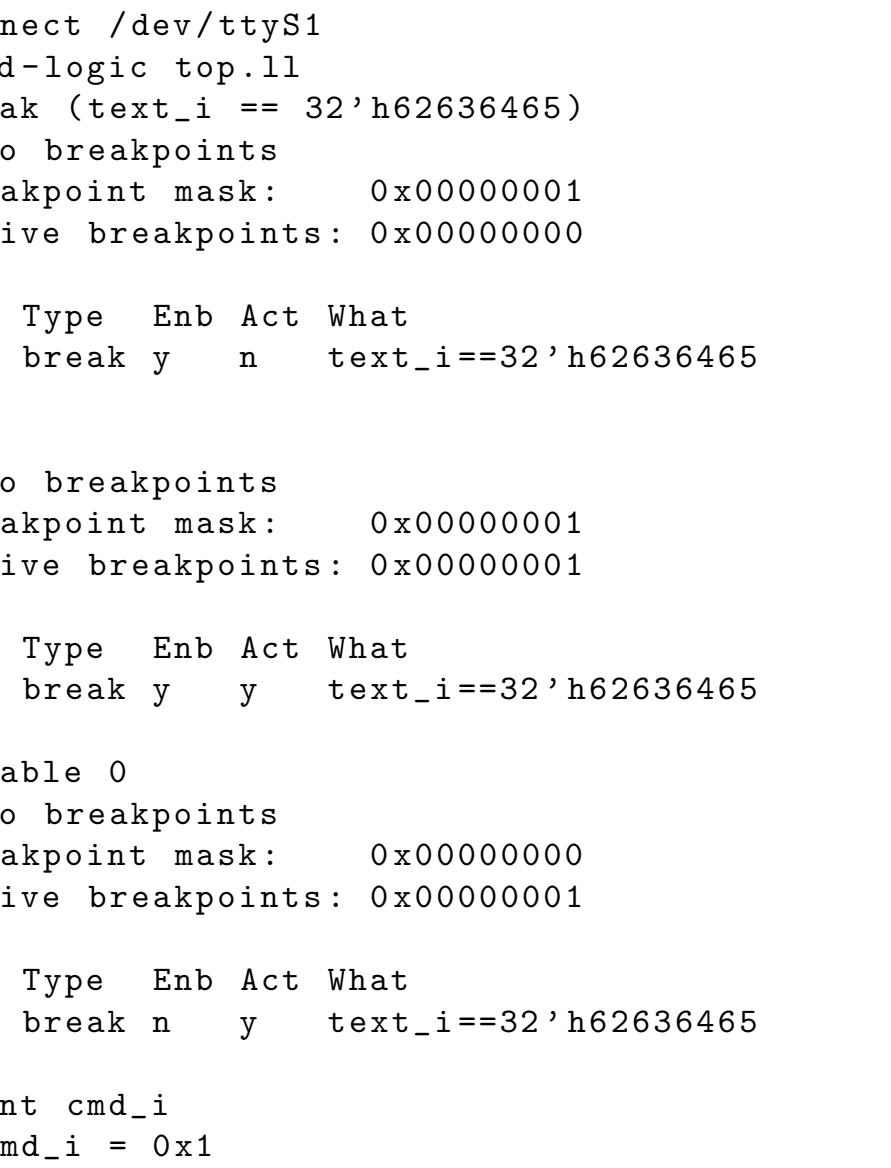

Listing 6.2: LLD debug transcript for a SHA-1 core.

\section{Software}

A transcript of a debug session is shown in Listing 6.2. The syntax and commands are similar to those found in the GNU gdb debugger. A debugging session is initiated by connecting to the on-chip processor through the serial line and then building the design and device model from the logic-allocation file. Reading the logic-allocation file is the single most time- and resource-intensive task and the primary reason for locating this operation to a conventional workstation. Even for small designs, logic-allocation files can be around $25 \mathrm{MB}$ and for a large design such as the OpenSPARC, can exceed 255 MB. 
Listing 6.2 shows how conditional breakpoints are first defined using top-level ports and signals defined in the breakpoint module. An information command is used to display breakpoint status. The information output begins with two status masks: the breakpoint mask shows which breakpoints are enabled, while the active breakpoint mask indicates which of the 32 breakpoints are currently active. This is followed by a detailed listing, shortened here for readability, which defines the index of the breakpoint, whether the breakpoint is a conditional breakpoint or an assertion, whether or not the breakpoint is active or enabled, and the original text of the breakpoint. As with conventional command-line debuggers, breakpoints can be individually enabled or disabled through a software-controlled mask without the need to rebuild or restart the design. The final print command in the listing demonstrates the ability to print the value of an arbitrary design register from the commandline.

Connections to the breakpoint logic region use FPGA routing resources out of necessity. Although this approach would be considered a shortcoming, DMD's restriction to top-level signals and the ability to arbitrarily read any design register through the processor did not present any of the expected problems such as routing issues. DMD is intended to be used as a low-level analysis tool, focusing on the signal interactions between design modules and the internal state that drives those signals. DMD's core is instantiated as part of the static region, meaning subsequent modifications of partitioned modules do not require lengthy rerouting. While a connectionless approach would have allowed any arbitrary register of the design to appear in a breakpoint condition and eliminated the need for an additional PR region, this would require a costly polling scheme of the targeted signals, reducing execution performance. LLD's ability to execute at the target design speed is unique among approaches that have full visibility of the design.

LLD breakpoints are implemented as asynchronous logic, signaling to the PDC to interrupt execution and allow software identification of active breakpoints. Each statement represents 
a breakpoint as entered at the command-line. Activated rules signal to the PDC which of the 32 breakpoints triggered and suspended execution. Clock buffers respond to control signals at the next rising edge, provided that setup and hold time requirements are met. If not, transition is deferred until the following rising edge, providing at most a two-cycle latency. Consistent one-cycle response times were observed when the design was halted by breakpoints which was validated by checking register values against the programmed breakpoint. A diagnostic clock cycle counter was also implemented to determine reaction time latencies.

Often, synthesis can optimize away logic, even when instructed not to using synthesis pragmas. This can be problematic if specifically trying to observe one of these signals. These optimizations are noted in the synthesis log files. However, it is still possible to use these signals as breakpoint conditions since these are top-level ports to the debug module and this prevents them from disappearing entirely.

Tool run times, particularly Map and PAR, were highly variable. The insertion of additional complex logic is expected to extend implementation times, but was sometimes observed to reduce it in unpredictable ways. For example, in large designs the addition of a ChipScope core is expected to lengthen the implementation time. However, as shown in Table 6.1, some implementation times were reduced, but still were several hours long. Even for a design partitioned through partial reconfiguration, the tools process (but do not implement) the entire design. While only the reconfigurable region is actually re-implemented in subsequent runs, the tools perform DRC on the entire design, lengthening run times particularly on large or complex designs. For instance, this proportionally lengthens bitfile generation time even though a smaller, partial bitstream is the only portion being generated anew.

To evaluate the efficiency of this approach, several common debugging tasks were performed. In many cases ChipScope may require a post-synthesis re-implementation of the design to 
accommodate a new task, dominated largely by Map and PAR runtime. However, once implemented with the LLD flow, different breakpoint scenarios were possible by only reimplementing the altered breakpoint region. With ChipScope it is not possible to arbitrarily halt the design and read a randomly selected register. The embedded logic analyzer and control cores must be reconfigured and re-implemented for a specific event. Using LLD, breakpoints were set to suspend execution so that registers could be inspected and then later the design was stepped by a small number of clock cycles to advance execution and continue inspection. LLD allows register values to be randomly read from the command-line using their full hierarchical design name in two to eight seconds depending on the width of the signal and distribution of the bits across the FPGA's configuration frames. While individual bits of a register are frequently placed as close as possible, it is usually not possible to find an entire register placed in a single configuration frame, thus resulting in fragmentation across several frames. One improvement was to group reads from the same frame into a single transaction by batching offsets from the same frame. This reduced read times by an average of $50 \%$. While additional improvements are possible, the two to eight seconds required for an arbitrary register represent a significant improvement over having to re-implement and reroute a design to change which registers should be targeted.

The console application was similarly evaluated for its performance. The most time-consuming recurring process was the on-chip portion of reading an FPGA register. Individually, each bit requires 0.5 seconds to process a read operation, however the locality of reference of adjacent bits in the same frame prevents this from being linearly proportional. A 32-bit register takes only 7.5 seconds due to the packing of the bits. Despite utilizing a serial console - one of the slowest communications mediums still being used in modern computing systems - the entire round-trip cost is only 0.5 seconds. The most time consuming non-recurring operation is that of reading the logic-allocation file. Approximately $50 \%$ of the CPU-time, accounting for 1.6 seconds of the application's startup time, is spent parsing the logic-allocation file to 
build the logic and device model. Figure 6.4, produced from the Google Perf Tools profiler suite, shows the relative time spent in a typical execution scenario. It is noteworthy that none of the blocking operations delegated to the FPGA are long enough to appear in the profiler's output as a significant source of execution time.

\subsubsection{Summary and Future Work}

LLD provides a software-based approach to both controlling and observing FPGA execution. An onboard processor provides an interactive user interface to the FPGA, which is in stark contrast to the post-execution, forensic-style approaches of vendor embedded logic analyzers. LLD provides an interface that is transparently distributed across a conventional workstation and the onboard processor of the FPGA to provide an all encompassing view of the device. This view is cross-referenced with design-time reports, such as the logic-allocation file, which can be leveraged to build symbol tables useful for debugging. No other debugging frameworks were identified that provide such an approach and allow the design to execute at its full speed.

Enhancements reserved for future work include extending the capabilities of the debug logic and improving the performance of the software. Currently, LLD only supports the generation of asynchronous debug logic which is a condition that can be active during a single clock cycle. Assertion-Based Verification, introduced in Section 2.3.4, allows sequences of states to be defined, equivalent to defining a state-machine that must be activated before the condition fires. A state-based approach to debugging would expand the scope of LLD to far more complex and complete scenarios.

Performance of the user application is another area reserved for future work. Although responsive, response times could be further improved. While the communication channel could be upgraded to a faster medium, such as USB or Ethernet, this would limit portability across development boards. A half-second latency in communication is tolerable, although 
not optimum. The greatest latency is found in process of gathering bits from the FPGA, which if not optimized, compounds the round-trip latency over the serial line. The current implementation sequentially processes the register bits, grouping subsequent requests to the same configuration frame before querying the FPGA-based processor and proceeding to the next frame. Separate and repeated requests are made to the same configuration frame if intermediate bits reside in other frames. A more efficient approach is to globally group all the requests to the same frame. A preliminary analysis shows that this approach could reduce the time required to read a 32-bit register by nearly half. 


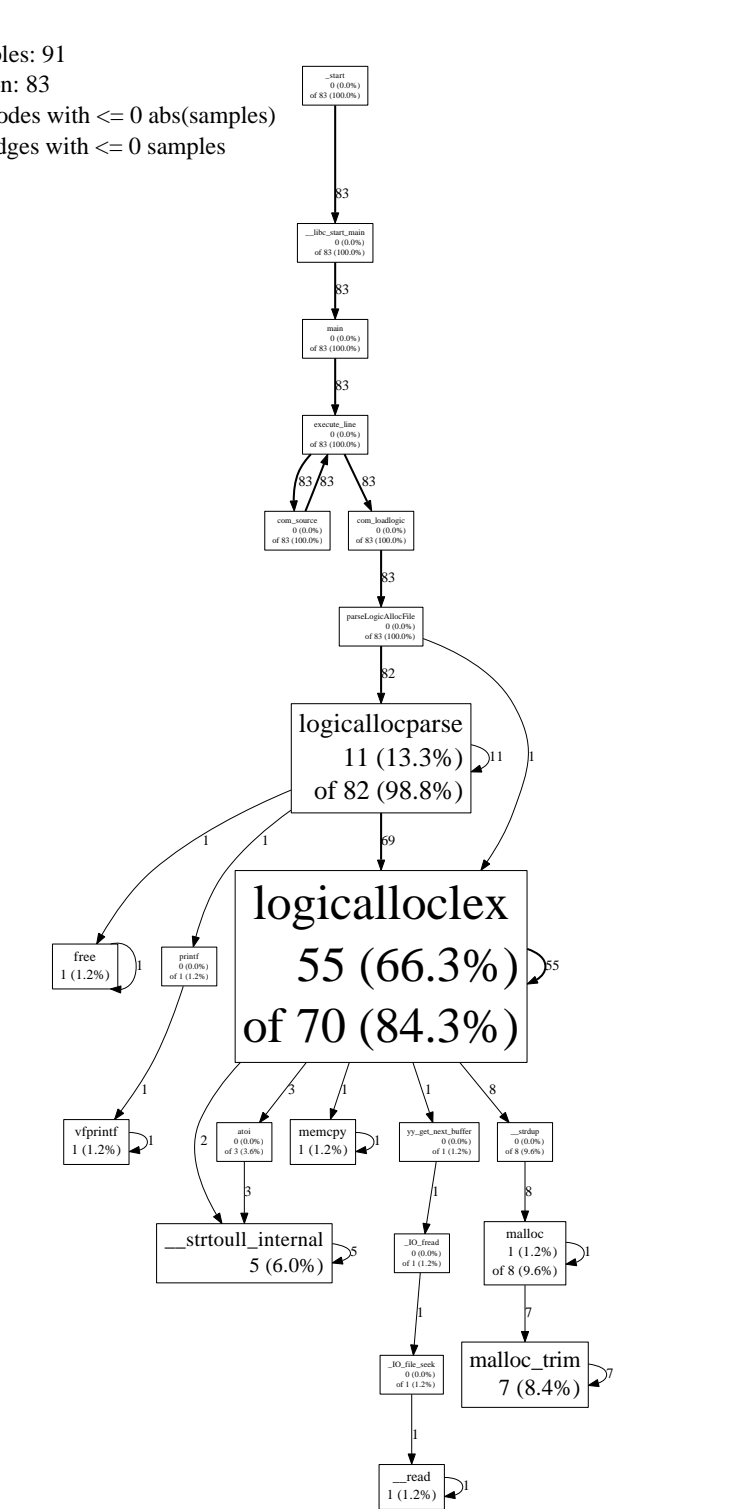

Figure 6.4: Execution call graph of console application. 


\section{Chapter 7}

\section{Conclusions and Future Work}

The lack of a structured development and debug lifecycle for FPGAs causes two issues. First, initial high-level language reference models become isolated from the implemented hardware resulting in the model verification gap. This makes validating hardware against the reference model a manual task. The second issue arises once the hardware has been implemented and is inaccessible to inspection and debug. This is in stark contrast to the flexible and open development environments found in software. The thesis of this dissertation is that the same high level of design visibility and control found in software development environments could be ported to FPGAs by incorporating software control directly into the FPGA. This was achieved by first identifying the primary difficulties in the conventional FPGA design flow, and developing embedded software-based solutions to improve accessibility and raise the abstraction level of these tasks.

\subsection{Review of Contributions}

The contributions of this research are as follows: 
- Development of a tool that addresses the model verification gap by raising the abstraction level for FPGA validation and debug.

FPGA debug has traditionally required a detailed knowledge not only of the design being developed, but also of the target hardware. The original RTL source is often too primitive to validate against high-level functionality. The HLV framework introduced in Section 4.1 allows synthesized hardware to be directly linked to a high-level language reference model without invasively modifying either one. The reference model and hardware are concurrently executed on the FPGA with the hardware design running at the target frequency. HLV serves as a software-based unit-testing framework for FPGA designs, capable of being automated to progressively test increasing levels of the design hierarchy with software-generated test vectors with the results being immediately validated. HLV is unique in that not only does it link hardware to software reference models, but also that the validation can be performed at the hardware's target execution frequency. Debugging against high-level models is not common even in HLS flows.

HLV is useful in that it removes the burden of manually validating reference models against the implemented hardware, even for design flows not destined for FPGAs. In most cases, hardware development and validation is repeated anew first for simulation development and then again for actual hardware which may draw from sources not used during simulation. This can open the door to unexpected and expensive design flaws. In the case of some ASIC design strategies and unlike FPGA development, none of the application HDL used in simulation can be applied. In most ASIC flows, FPGAs are used to logically and functionally validate the design before being sent to production or "taped-out". The Pentium FDIV bug $[80,81]$ is one such example of the consequences of the gap between reference model and implementation validation that HLV aspires to 
address. HLV can also be applied to HLS flows which still have not eclipsed traditional HDL development. HLV can be used to validate HLS-generated designs since the machine-generated RTL does not lend itself well to traditional debugging or formal verification.

- Development of a tool that improves visibility, control, and agility for FPGA debug by introducing software development environment facilities.

Unlike software development, FPGAs have few means to inspect or debug a running design such as the console messages or comprehensive debuggers that exist in software to provide detailed analysis of the design or halt execution. The internal state elements of the design have limited or no visibility, and execution occurs at millions of clock cycles per second. An error may occur and by the time it manifests itself, the state of the device may have changed so much that its diagnosis is impossible. An approach to improve visibility, control, and the agility of an FPGA development environment was introduced in Section 4.2 and implemented in Section 5.2. The developed framework brings some of the useful facilities found in software development environments to FPGAs. The system presented here allows a running, implemented design to be controlled and inspected at its target speed with the interface and agility of software development tools. While most FPGA development tools place the debugger's viewpoint outside the design, the developed framework places it within the FPGA, transforming the storage elements of the reconfigurable fabric to a conventional memory. Symbolic access to design elements is enabled by the construction of a symbol table not unlike those found in software debuggers. The agility of software breakpoints is recreated in a partially reconfigurable region that can be quickly implemented without affecting the rest of the design. 


\section{$7.2 \quad$ Future Work}

The research presented here shows that software is a viable means to both validating and debugging FPGA designs despite the wide disparity in performance. Several enhancements and optimizations were reserved for future work, such as functional and performance-related improvements.

The HLV framework is currently programmed through manual transcription from simulation models. This is both time-consuming and error-prone and ultimately unnecessary with additional means to directly generate testbenches from simulations. HLV can also be extended to handle more complex scenarios beyond fixed-latency transactions by incorporating programmable hardware that can interact with the design without the need to buffer signal traces. Other approaches could enable complex, software-controlled interactions capable of scripting large volumes of data, but might eliminate the sustained, full-speed execution demonstrated here.

LLD demonstrated fine-grain and interactive control over an implemented design through embedded software. Given the extensive use of multiple clock domains, there remains some research to be done in implementing cascading clock control logic to reliably synchronize all clock domains. The LLD software could be further improved in the areas of performance and efficiency. Operations requiring round-trip transactions cumulatively add to the total time needed, however methods have been identified that could reduce this time by as much as $50 \%$. This can be achieved by further optimizing the algorithms used to schedule reading and assembling design registers. Assertion-Based Verification techniques, such as those introduced in Section 2.3.4, which can define multi-state transitions for a single assertion, could be introduced to realize complex scenarios such as state-machine validation. 


\section{Bibliography}

[1] M. Wirthlin, B. Nelson, B. Hutchings, P. Athanas, and S. Bohner, "A Research Agenda for Improving Configurable Computing Design Productivity," NSF Center for High-Performance Reconfigurable Computing (CHREC), Salt Lake City, UT, Tech. Rep., June 2008. [Online]. Available: http://www.chrec.org/ftsw/BYU_VT_Report.pdf

[2] B. Nelson, M. Wirthlin, B. Hutchings, P. Athanas, and S. Bohner, "Design Productivity for Configurable Computing." CSREA Press, 2008, pp. 57-66.

[3] Xilinx, Inc., "UG190: Virtex-5 FPGA User Guide."

[4] —, "XC4000E and XC4000X Series Field Programmable Gate Arrays (Product Specification)."

[5] — , "XC5200 Series Field Programmable Gate Arrays (Product Specification)."

[6] _ _ "DS003: Virtex 2.5 V Field Programmable Gate Arrays (Product Specification)."

[7] — , "DS031: Virtex-II Platform FPGAs: Complete Data Sheet."

[8] — , "DS083: Virtex-II Pro and Virtex-II Pro X Platform FPGAs: Complete Data Sheet."

[9] —_, "DS100: Virtex-5 Family Overview."

[10] — _ "DS112: Virtex-4 Family Overview." 
[11] — , "DS150: Virtex-6 Family Overview."

[12] — _ "DS180: 7 Series FPGAs Overview."

[13] C. Metz, "Dennis Ritchie: The Shoulders Steve Jobs Stood On," Wired, October 2011. [Online]. Available: http://www.wired.com/wiredenterprise/2011/10/thedennisritchieeffect/

[14] M. Santarini, "Driver assistance revs up on xilinx fpga platforms," Xcell Journal, vol. 66, 2008.

[15] J. Wilcox and M. Kanellos, "Amd makes move to 1-ghz chip," CNET News, March 2000. [Online]. Available: http://news.cnet.com/2100-1040-237615.html

[16] C. Schalick, "Debugging FPGA designs may be harder than you expect," EDN, vol. 54, no. 21, p. 23, 2009.

[17] Free Software Foundation, "GNU Make." [Online]. Available: http://www.gnu.org/software/make

[18] Altera Corporation. Quartus II Incremental Compilation for Hierarchical and TeamBased Design. [Online]. Available: http://www.altera.com/literature/hb/qts/qts_ qii51015.pdf

[19] Development System Reference Guide, v10.1 ed., Xilinx, Inc., 2008. [Online]. Available: http://www.xilinx.com/itp/xilinx4/pdf/docs/dev/dev.pdf

[20] T. Frangieh, A. Chandrasekharan, S. Rajagopalan, Y. Iskander, S. Craven, and C. Patterson, "PATIS: using partial configuration to improve static FPGA design productivity," in 17th Reconfigurable Architectures Workshop (RAW), 2010.

[21] XST User Guide, v11.3 ed., Xilinx, Inc., September 2009. 
[22] Xilinx, Inc. Memory Recommendations. [Online]. Available: http://www.xilinx.com/ise/products/memory.htm

[23] N. Steiner. ChipScope scripting for batch data collection? [Online]. Available: usenet://comp.arch.fpga

[24] Xilinx, Inc., "MicroBlaze Processor Reference Guide."

[25] L. Wirbel, "FPGA survey sees sunset for gate arrays, continued dominance by Xilinx, Altera," EE Times, October 2008. [Online]. Available: http://www.eetimes.com/showArticle.jhtml?articleID=211200184

[26] "IEEE Standard for Verilog Hardware Description Language," IEEE Std 1364-2005 (Revision of IEEE Std 1364-2001), pp. 1-560, 2006.

[27] "IEEE Standard VHDL Language Reference Manual," IEEE Std 1076-2008 (Revision of IEEE Std 1076-2002), pp. c1-626, 262009.

[28] "IEEE Standard System C Language Reference Manual," IEEE Std 1666-2005, pp. 1 $-423,2006$.

[29] "IEEE Standard for System Verilog-Unified Hardware Design, Specification, and Verification Language," IEEE STD 1800-2009, pp. C1 -1285, 2009.

[30] Cadence Design Systems, Inc. Cadence e Verification Language. [Online]. Available: http://www.cadence.com/Alliances/languages/Pages/e_page.aspx

[31] "IEEE Standard for the Functional Verification Language e," IEEE Std 1647-2011 (Revision of IEEE Std 1647-2008), pp. 1 -495, 262011.

[32] BlueSpec, Inc. BlueSpec Language. [Online]. Available: http://www.bluespec.com/ 
[33] P. Coussy, D. D. Gajski, M. Meredith, and A. Takach, "An Introduction to High-Level Synthesis," Design \& Test of Computers, IEEE, vol. 26, no. 4, pp. 8-17, 2009.

[34] S. Sarkar, S. Dabral, P. K. Tiwari, and R. S. Mitra, "Lessons and Experiences with High-Level Synthesis," Design $\&$ Test of Computers, IEEE, vol. 26, no. 4, pp. 34-45, 2009.

[35] E. Eto, Xilinx Application Note 290: Difference-Based Partial Reconfiguration, v2.0 ed., Xilinx, December 2007. [Online]. Available: http://www.xilinx.com/support/documentation/application_notes/xapp290.pdf

[36] S. D. Craven, "Structured approach to dynamic computing application development," Ph.D. dissertation, Virginia Polytechnic Institute and State University, Blacksburg, VA, USA, 2008.

[37] G. Subbarayan, "Automatic instantiation and timing-aware placement of bus macros for partially reconfigurable FPGA designs." Master's thesis, Virginia Polytechnic Institute and State University, Blacksburg, VA, USA, 2010.

[38] A. Chandrasekharan, "Accelerating incremental floorplanning of partially reconfigurable designs to improve FPGA productivity." Master's thesis, Virginia Polytechnic Institute and State University, Blacksburg, VA, USA, 2010.

[39] "IEEE Standard for Reduced-Pin and Enhanced-Functionality Test Access Port and Boundary-Scan Architecture," IEEE Std 1149.7-2009, pp. c1 -985, 102010.

[40] Xilinx, Inc., UG012: Virtex-II Pro and Virtex-II Pro X FPGA User Guide, v4.2 ed., Xilinx, Inc., November. [Online]. Available: http://www.xilinx.com/support/documentation/user_guides/ug012.pdf 
[41] UG071: Virtex-4 FPGA Configuration User Guide, v1.11 ed., Xilinx, Inc., June 2009. [Online]. Available: http://www.xilinx.com/support/documentation/user_ guides/ug071.pdf

[42] UG191: Virtex-5 FPGA Configuration User Guide, v9.0 ed., Xilinx, Inc., January 2008. [Online]. Available: http://www.xilinx.com/support/documentation/user guides/ug191.pdf

[43] UG360: Virtex-6 FPGA Configuration User Guide, v3.4 ed., Xilinx, Inc., November 2011. [Online]. Available: http://www.xilinx.com/support/documentation/user_ guides/ug360.pdf

[44] UG470: 7 Series FPGAs Configuration User Guide, v1.3 ed., Xilinx, Inc., February 2012. [Online]. Available: http://www.xilinx.com/support/documentation/user guides/ug470_7Series_Config.pdf

[45] Xilinx, "Xilinx ChipScope Pro." [Online]. Available: http://www.xilinx.com/tools/cspro.htm

[46] Altera Corporation, "Design Debugging Using the SignalTap II Embedded Logic Analyzer."

[47] GateRocket, Inc., "GateRocket Product Overview," 2008.

[48] "FPGA tool vendor gaterocket folds," EE Times, August 2011. [Online]. Available: http://www.eetimes.com/electronics-news/4218492/FPGA-tool-vendor-GateRocket-folds

[49] Sandbyte Technologies. FPGAXpose Data Sheet. [Online]. Available: http://www.sandbyte.com/FPGAXposeDataSheet.pdf

[50] Synopsys, Inc., "Fast, Efficient RTL Debug for Programmable Logic Designs." 
[51] Cadence Design Systems, Inc., "Cadence Palladium."

[52] W. Danghui, G. Deyuan, and L. Tao, "Breakpoint debugging mechanism for microprocessor design," 2003, pp. 456-458.

[53] A. Penttinen, R. Jastrzebski, R. Pollanen, and O. Pyrhonen, "Run-Time Debugging and Monitoring of FPGA Circuits Using Embedded Microprocessor," Design and Diagnostics of Electronic Circuits and systems, IEEE, pp. 147-148, 2006.

[54] Z. Baker and J. Monson, "In-situ FPGA debug driven by on-board microcontroller," in Field Programmable Custom Computing Machines, 2009. FCCM '09. 17th IEEE Symposium on, April 2009, pp. $219-222$.

[55] R. Kuramoto, "Application Note 058: Xilinx In-System Programming Using an Embedded Microcontroller." [Online]. Available: http://www.xilinx.com/support/documentation/application_notes/xapp058.pdf

[56] B. Hutchings, P. Bellows, J. Hawkins, S. Hemmert, B. Nelson, and M. Rytting, "A cad suite for high-performance fpga design," in Field-Programmable Custom Computing Machines, 1999. FCCM '99. Proceedings. Seventh Annual IEEE Symposium on, 1999, pp. $12-24$.

[57] "JHDL: FPGA CAD Tools." [Online]. Available: http://www.jhdl.org

[58] C. Patterson and S. Guccione, "Jbits design abstractions," in Field-Programmable Custom Computing Machines, 2001. FCCM '01. The 9th Annual IEEE Symposium on, 29 2001-april 2 2001, pp. $251-252$.

[59] P. Graham, B. Nelson, and B. Hutchings, "Instrumenting Bitstreams for Debugging FPGA Circuits," in Proceedings of the the 9th Annual IEEE Symposium on Field-Programmable Custom Computing Machines. Washington, 
DC, USA: IEEE Computer Society, 2001, pp. 41-50. [Online]. Available: http://portal.acm.org/citation.cfm?id=1058426.1058867

[60] D. Levi and S. A. Guccione, "BoardScope: A debug tool for reconfigurable systems," in Configurable Computing: Technology and Applications, Proc. SPIE 3526, J. Schewel, Ed. Bellingham, WA: SPIE - The International Society for Optical Engineering, November 1998, pp. 239-246.

[61] T. Price, D. Levi, and S. A. Guccione, "Debug of reconfigurable systems," Society of Photo-Optical Instrumentation Engineers (SPIE) Conference Series, vol. 4212, pp. 181-187, 2000.

[62] Impulse Accelerated Technologies, "Impulse CoDeveloper C-to-FPGA Tools." [Online]. Available: http://www.impulseaccelerated.com/products_universal.htm

[63] M. Gokhale, J. Stone, J. Arnold, and M. Kalinowski, "Stream-oriented FPGA computing in the Streams-C high level language," in Field-Programmable Custom Computing Machines, 2000 IEEE Symposium on, 2000, pp. 49 -56.

[64] "The Stanford SUIF Compiler Group." [Online]. Available: http://suif.stanford.edu

[65] D. Pellerin and S. Thibault, Practical FPGA programming in C. Upper Saddle River, NJ: Prentice Hall Professional Technical Reference, 2005.

[66] J. Curreri, G. Stitt, and A. George, "High-level synthesis techniques for in-circuit assertion-based verification," in Parallel Distributed Processing, Workshops and Phd Forum (IPDPSW), 2010 IEEE International Symposium on, april 2010, pp. 1 -8.

[67] Xilinx, Inc., "Partial Reconfiguration User Guide." [Online]. Available: http://www.xilinx.com/support/documentation/sw_manuals/xilinx12_1/ug702.pdf 
[68] A. Chandrasekharan, S. Rajagopalan, G. Subbarayan, T. Frangieh, Y. Iskander, S. Craven, and C. Patterson, "Accelerating FPGA development through the automatic parallel application of standard implementation tools," in Field-Programmable Technology (FPT), 2010 International Conference on, December 2010, pp. 53-60.

[69] S. Raja Gopalan, "Timing-aware automatic floorplanning of partially reconfigurable designs for accelerating FPGA productivity." Master's thesis, Virginia Polytechnic Institute and State University, Blacksburg, VA, USA, 2010.

[70] Xilinx, Inc., "DS449: LogiCORE IP Fast Simplex Link (FSL) V20 Bus."

[71] M. Shelburne, C. Patterson, P. Athanas, M. Jones, B. Martin, and R. Fong, "Metawire: Using FPGA configuration circuitry to emulate a network-on-chip," in Field Programmable Logic and Applications, 2008. FPL 2008. International Conference on, Sept. 2008, pp. $257-262$.

[72] "GDB: The GNU Project Debugger." [Online]. Available: http://www.gnu.org/software/gdb/gdb.html

[73] "GNU Readline Library." [Online]. Available: http://www.gnu.org/software/readline

[74] D. R. Stinson, Cryptography Theory and Practice, 3rd ed. Chapman \& Hall/CRC, 2006.

[75] "SHA-1 Collision Search Graz." [Online]. Available: http://boinc.iaik.tugraz.at/

[76] "OpenCores." [Online]. Available: http://www.opencores.org

[77] J. Curreri, G. Stitt, and A. George, "High-level synthesis of in-circuit assertions for verification, debugging, and timing analysis," International Journal of Reconfigurable Computing, vol. 2011, 2011. 
[78] Oracle Corporation, "OpenSPARC." [Online]. Available: http://www.opensparc.net/

[79] "FloPoCo." [Online]. Available: http://flopoco.gforge.inria.fr/

[80] R. P. Colwell, The Pentium chronicles: the people, passion, and politics behind Intel's landmark chips. Hoboken, N.J.: John Wiley \& Sons, Inc., 2006.

[81] D. Price, "Pentium FDIV flaw-lessons learned," Micro, IEEE, vol. 15, no. 2, pp. 86 -88, Apr 1995. 


\section{Appendix A}

\section{Open-Source and Free Software Acknowledgement}

The following open-source or free software packages were instrumental in this research. Links to software homepages and/or source are included for reference.

- Ubuntu GNU/Linux. http://www.ubuntu.com.

- Flex lexer, an open-source successor to the lex lexer generator. http://flex.sourceforge.net.

- Bison parser generator, an open-source successor to the yacc parser generator. http://www.gnu.org/software/bison.

- Vim Editor. http://www.vim.org.

- Oracle VirtualBox virtualization software. http://www.virtualbox.org.

- GNU Readline Library. http://www.gnu.org/software/readline.

- Apache Xerces XML Library. http://xerces.apache.org. 


\section{Appendix B}

\section{Source Code}

\section{B.1 High-Level Validation}

Listing B.1: HLV User Logic.

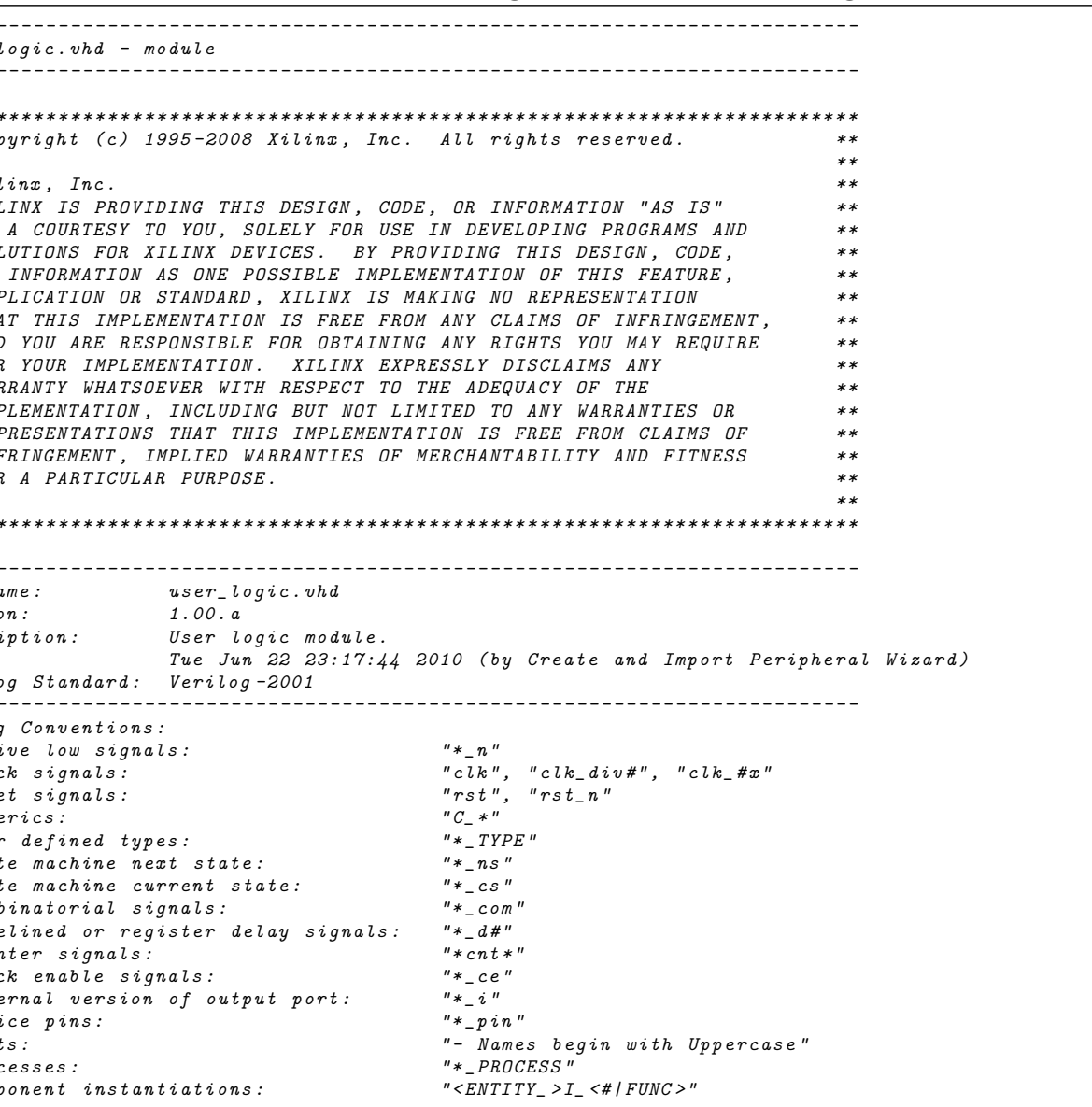




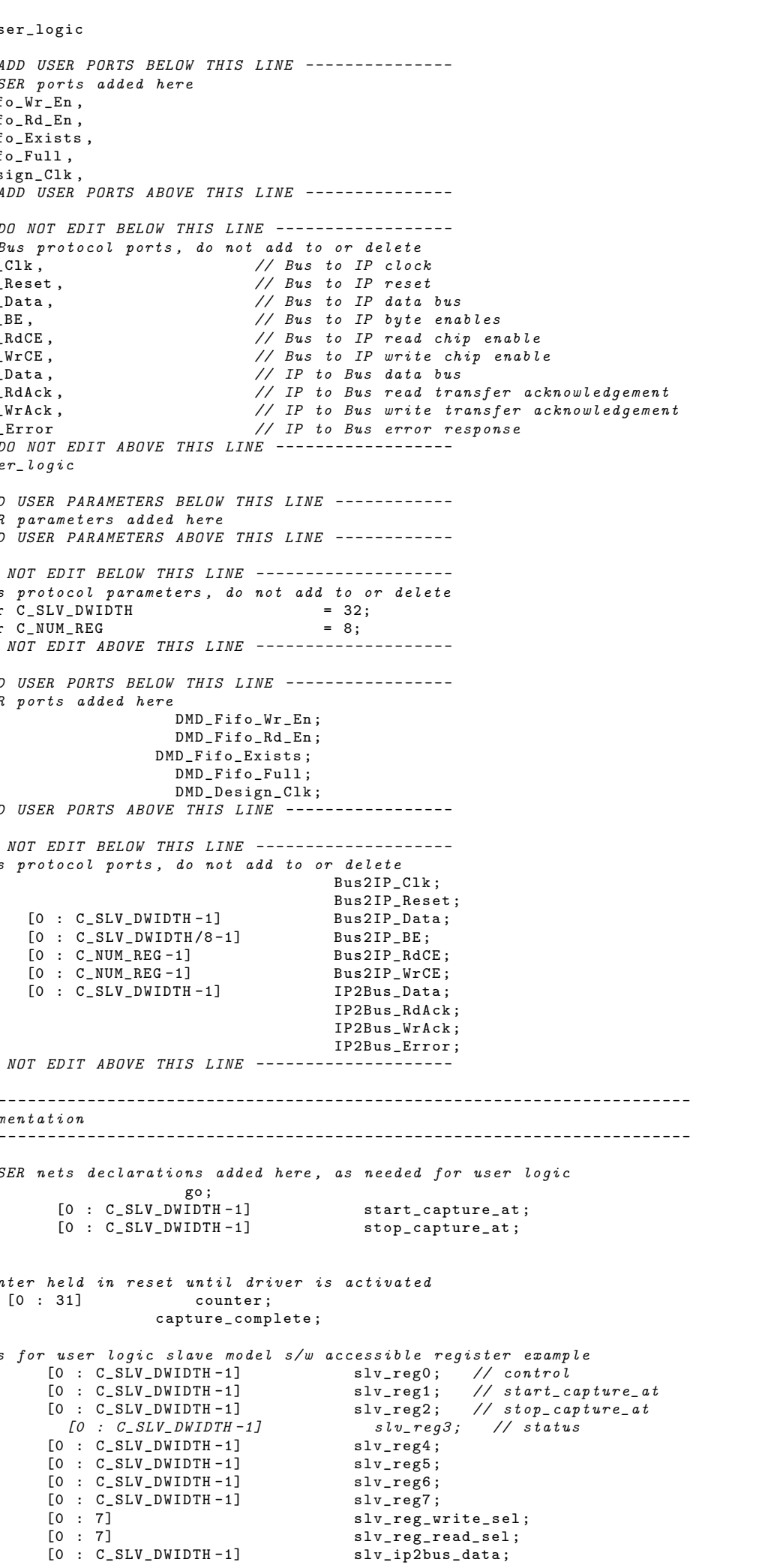




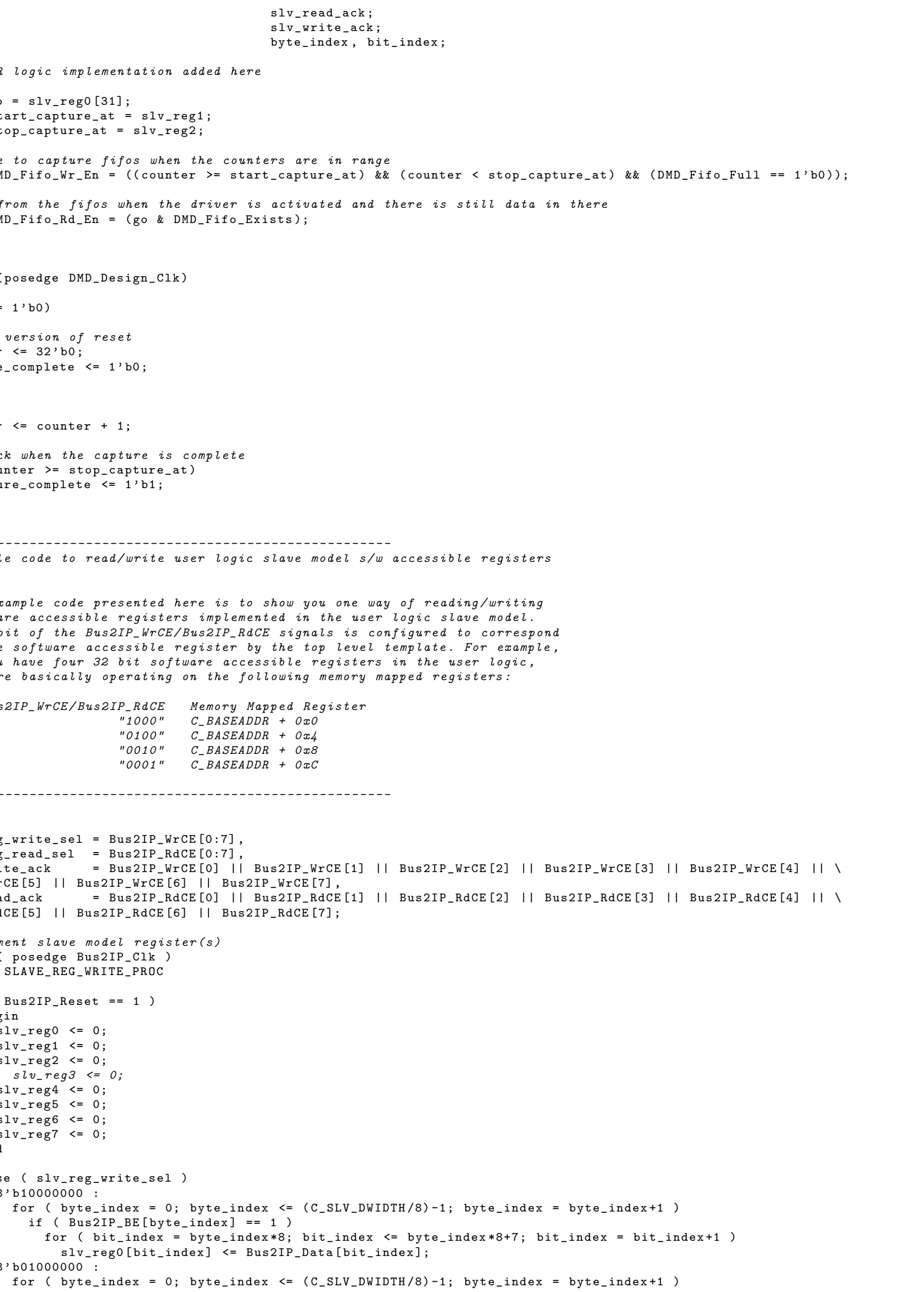




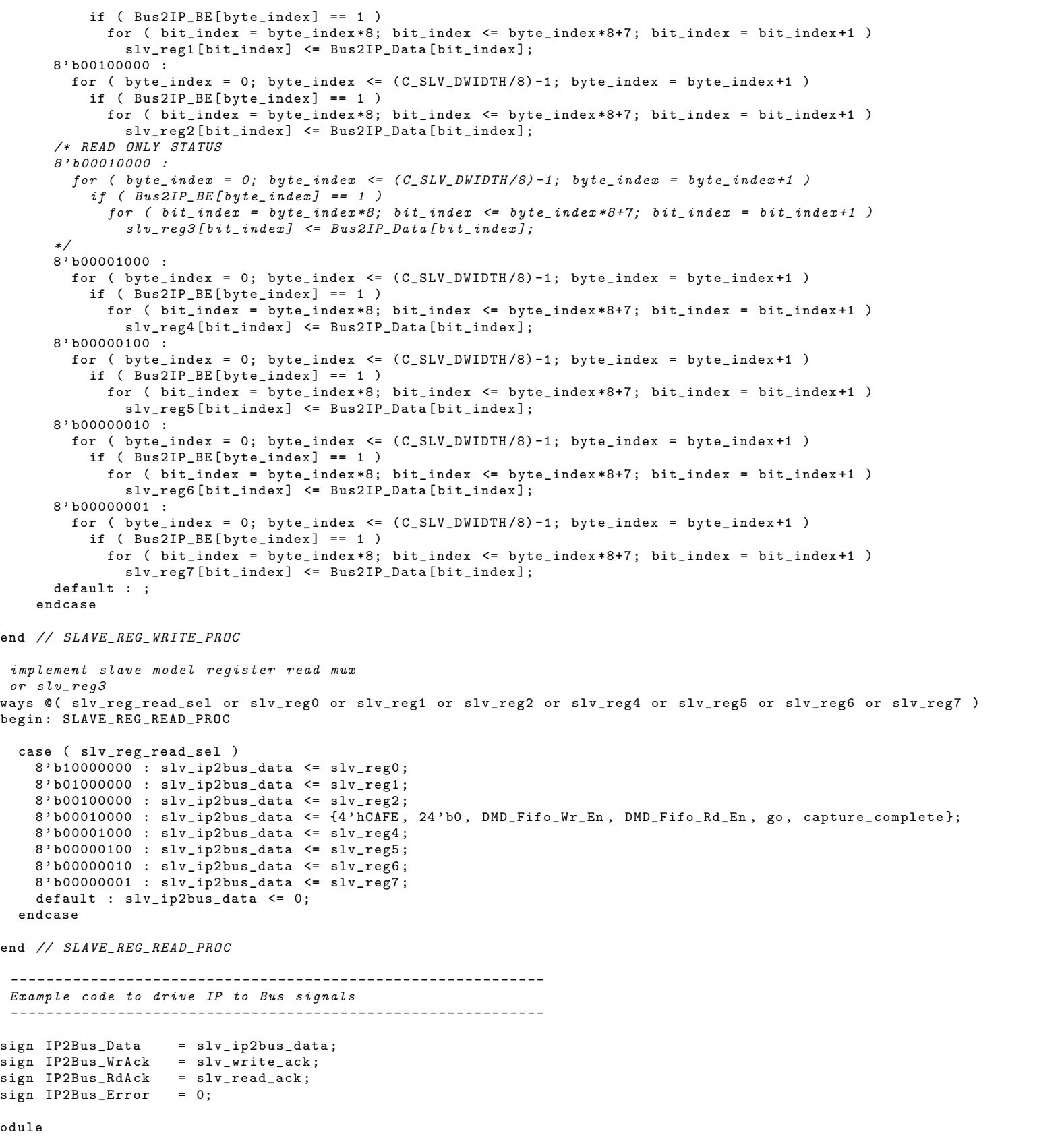


Listing B.2: SHA Test Code.

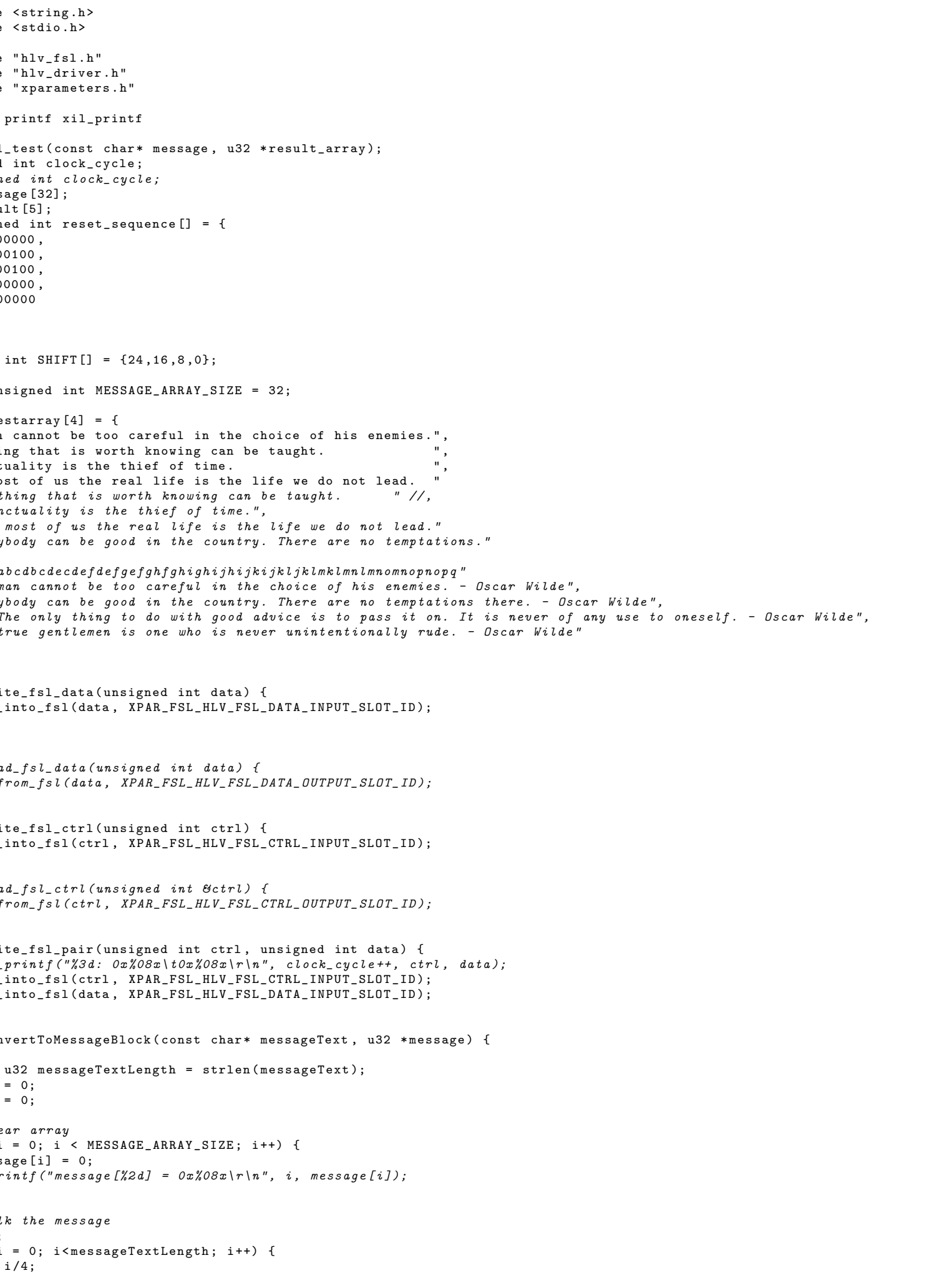




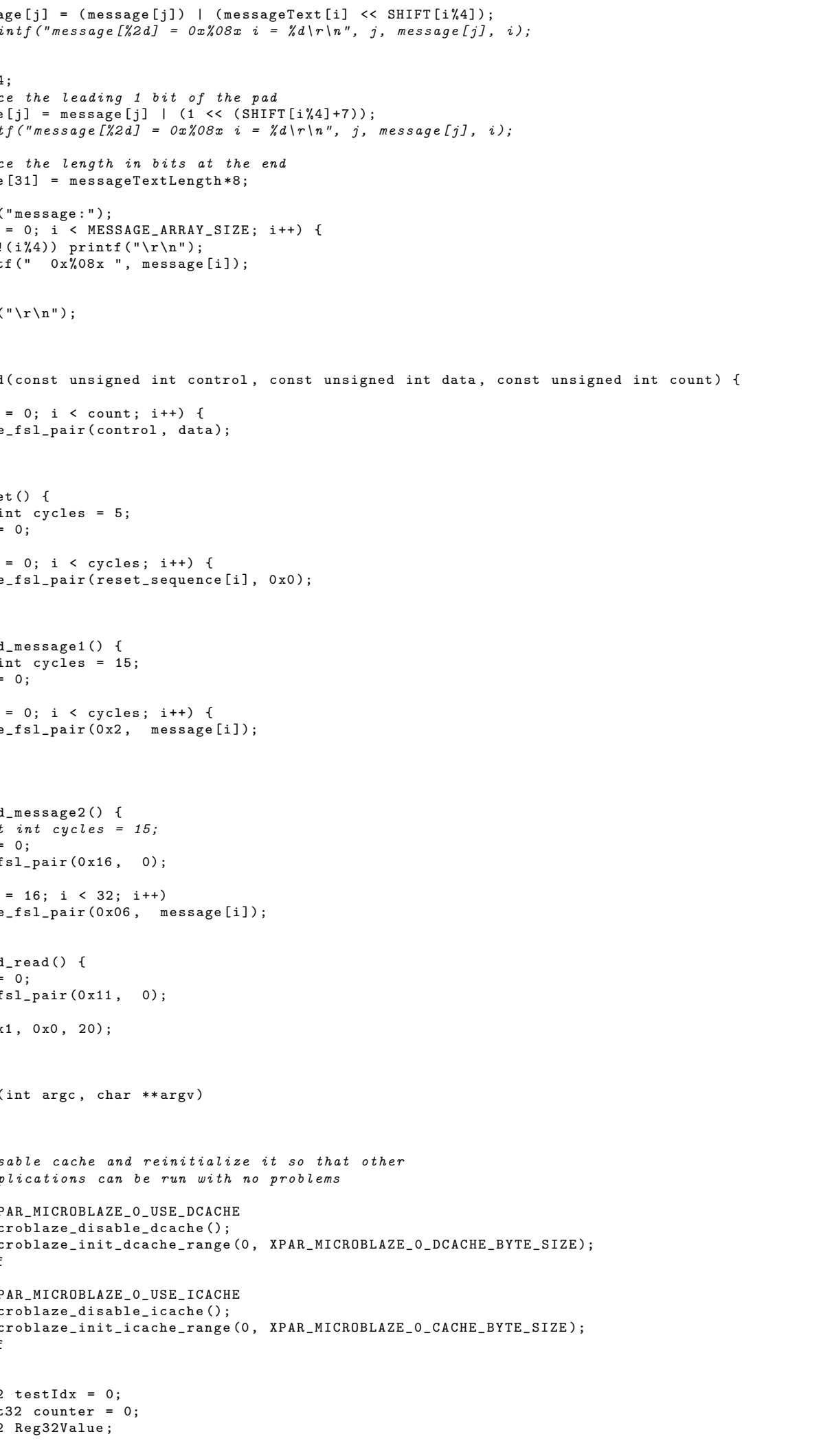




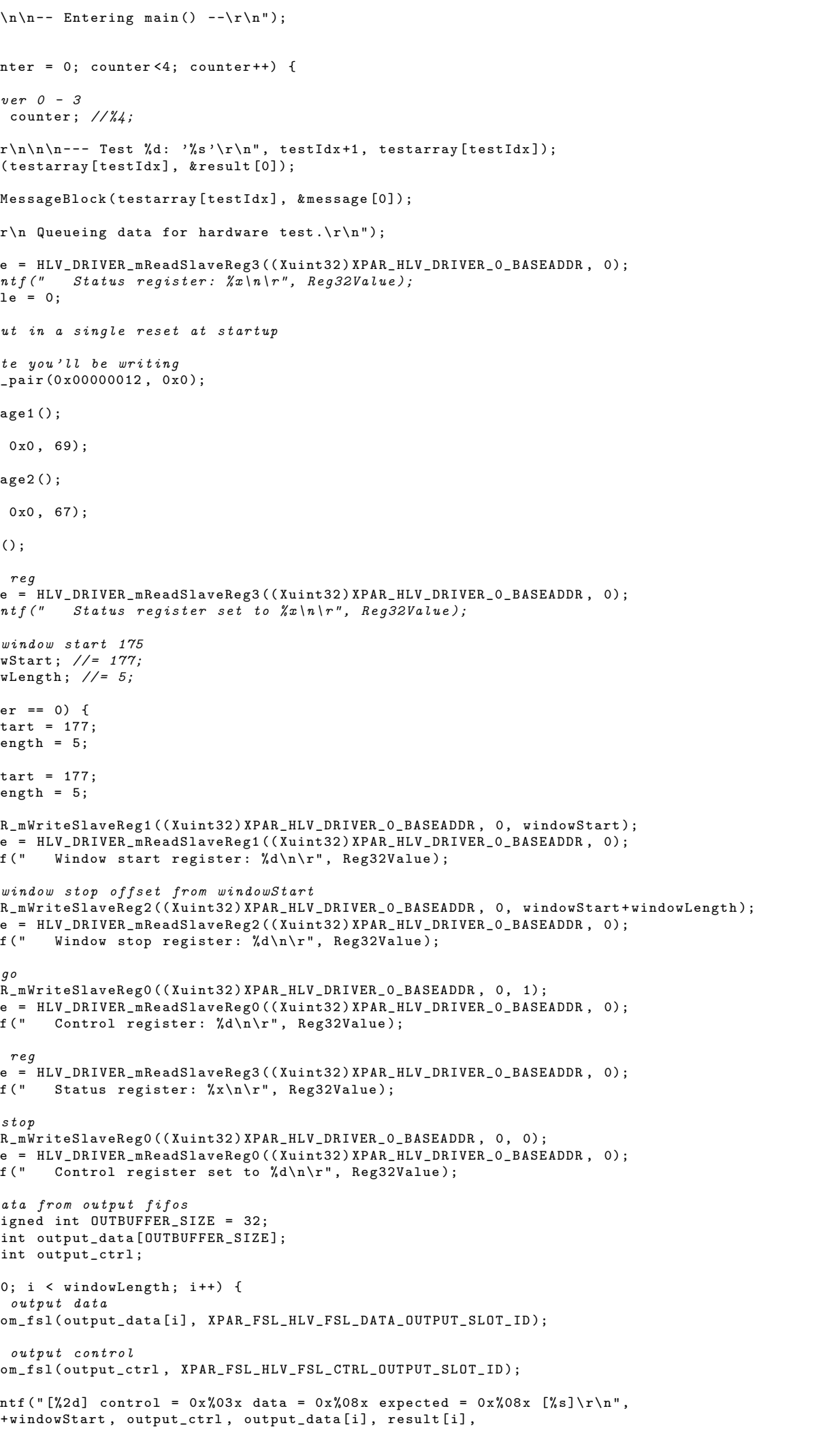


Yousef S. Iskander

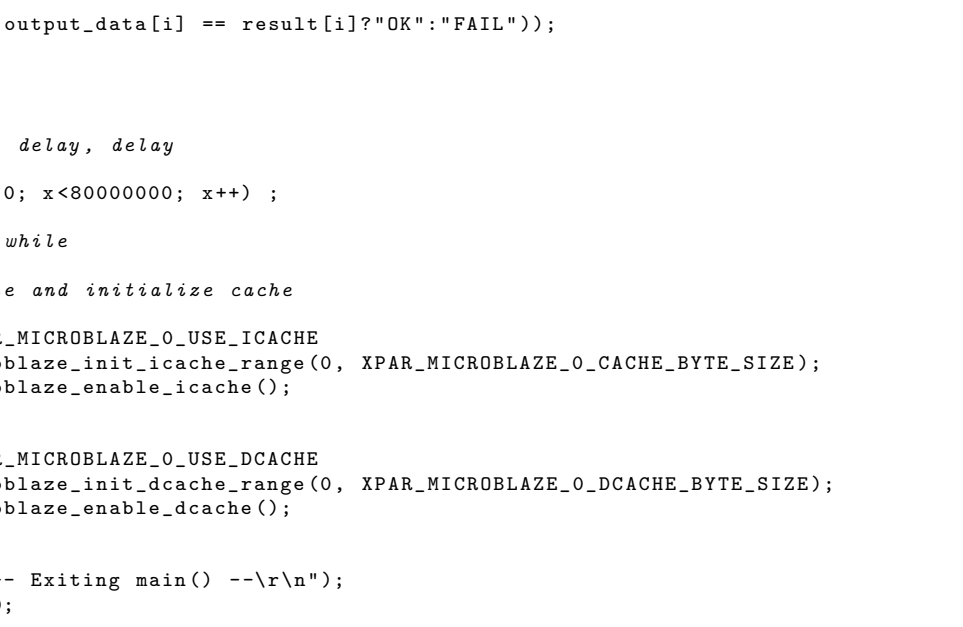


Listing B.3: SHA Test Code.

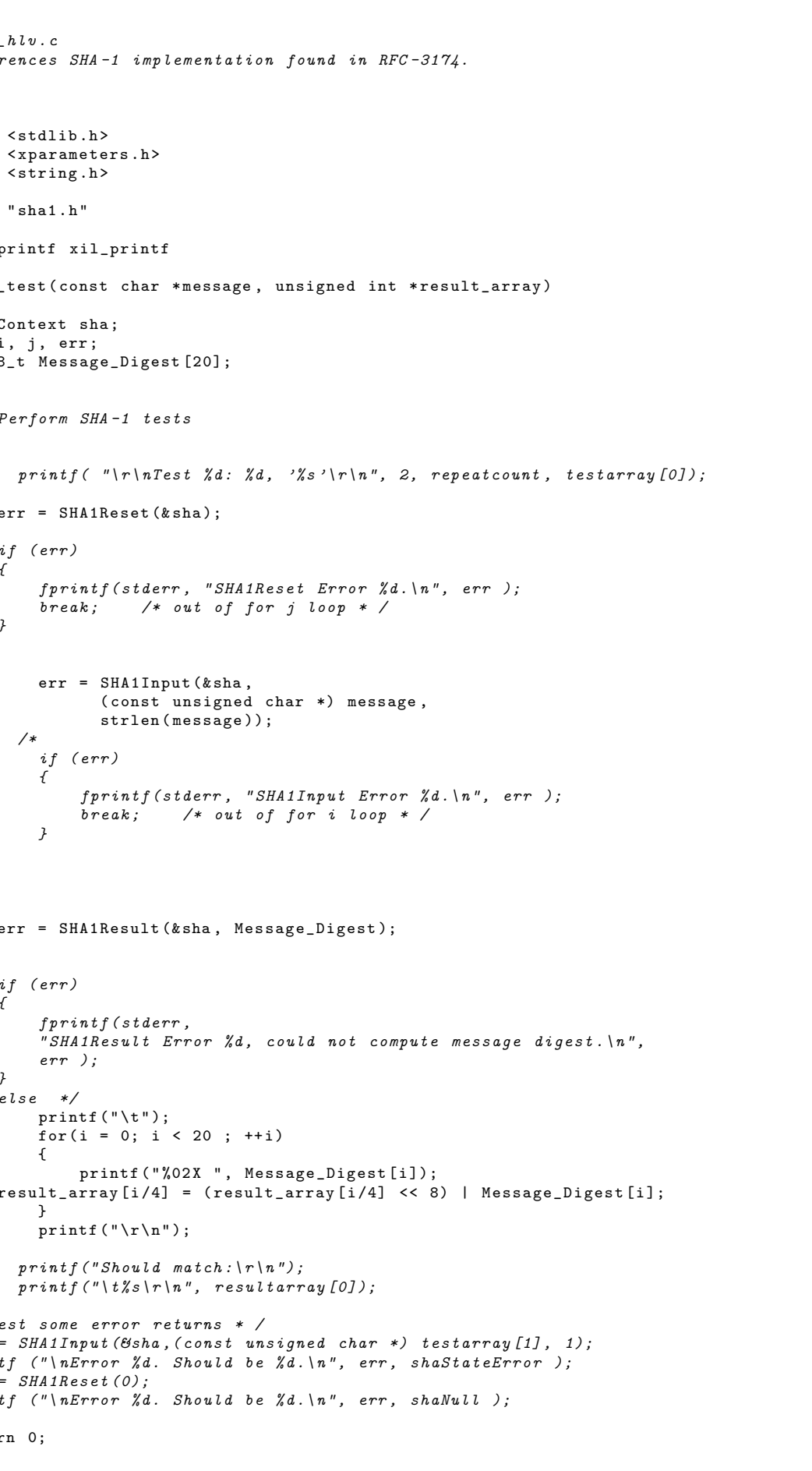


Yousef S. Iskander

\section{B.2 Low-Level Debug}

Listing B.4: Logic Allocation Data Structure Definition Header.

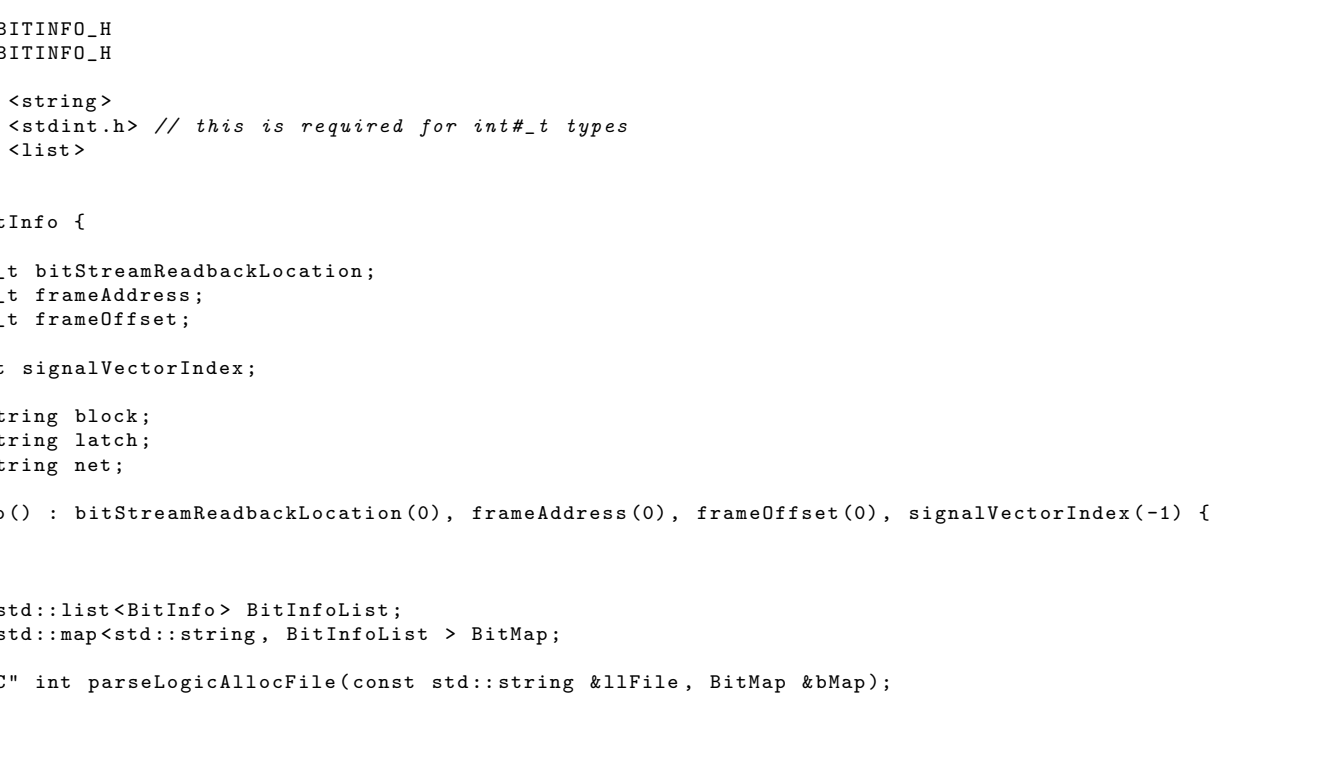


Yousef S. Iskander

Listing B.5: Breakpoint Data Structure Header.

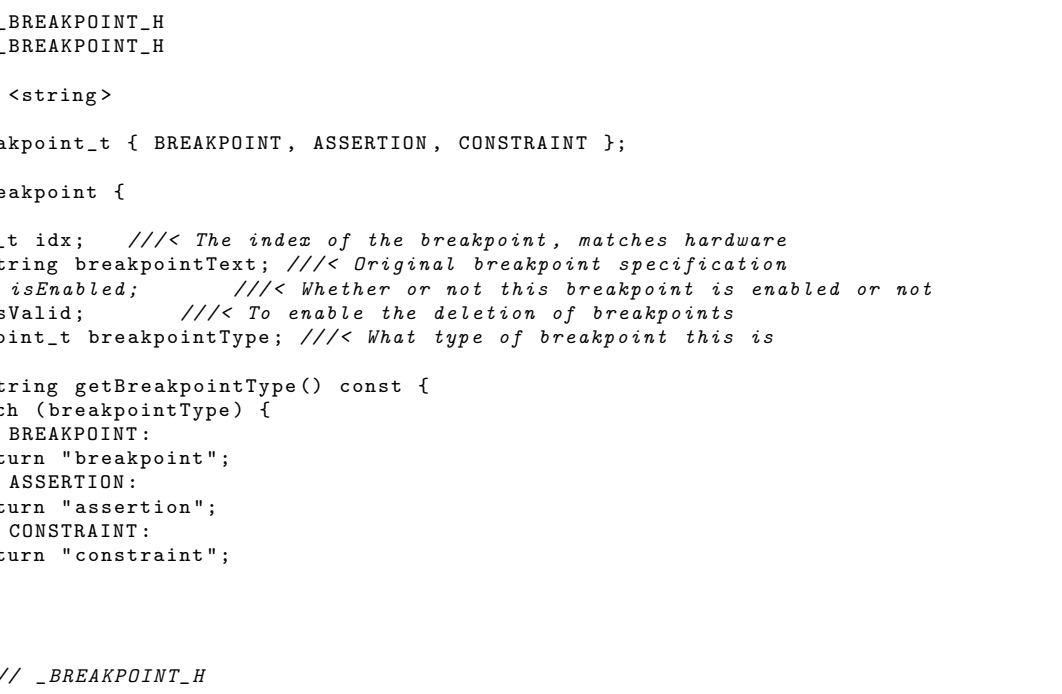


Listing B.6: Serial Communications Source.

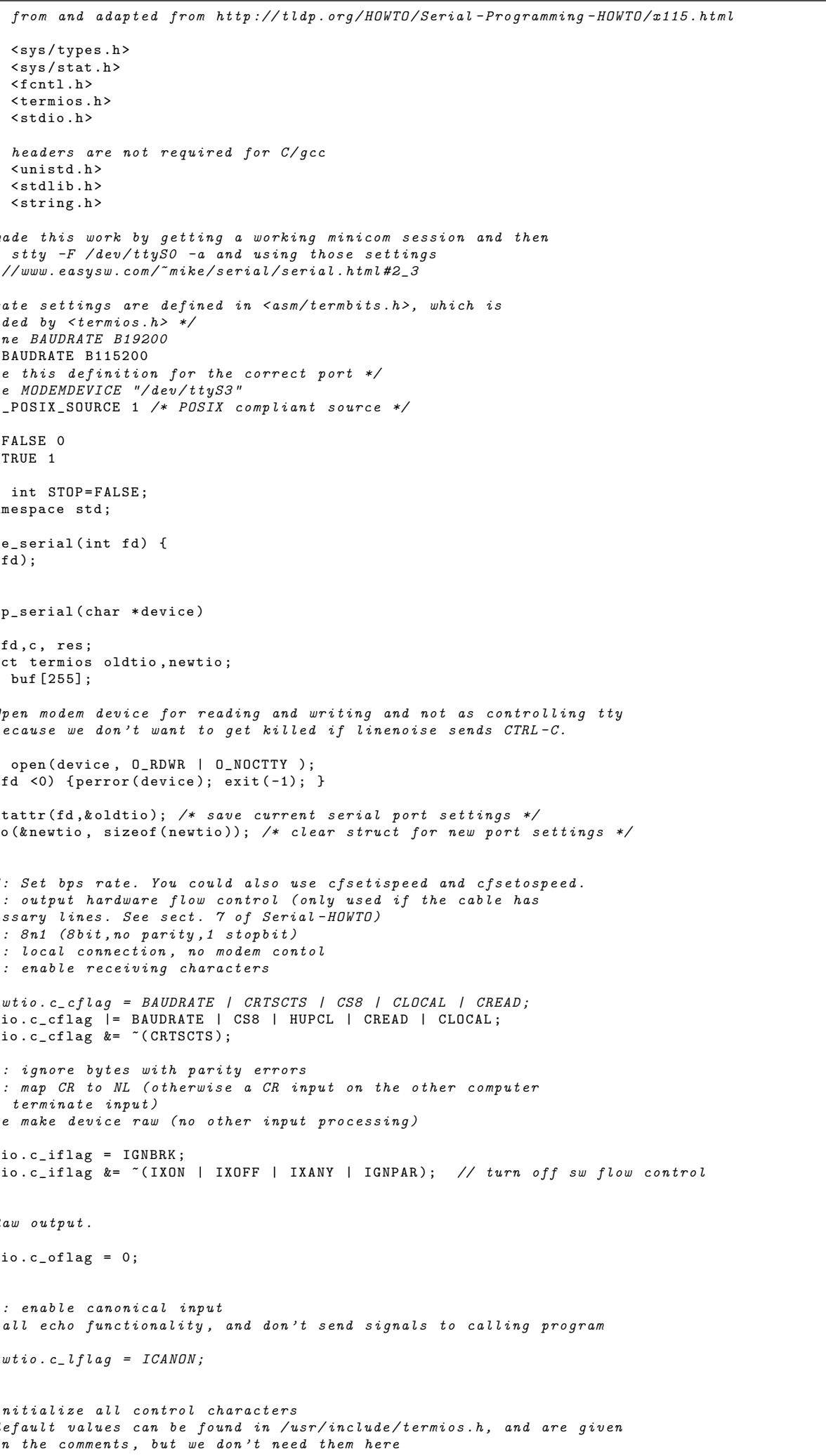


Yousef S. Iskander

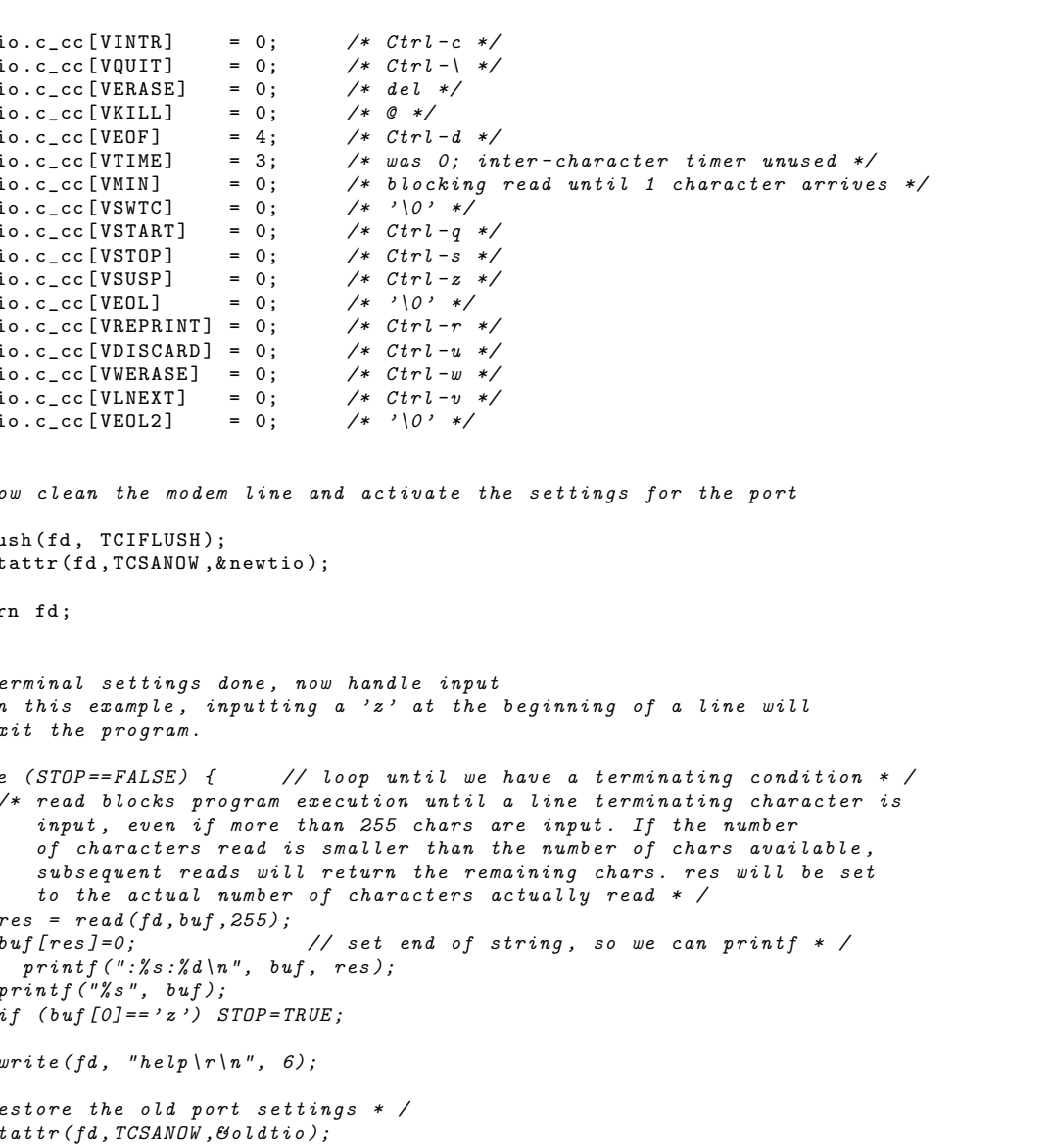


Listing B.7: Debugger Commands Header.

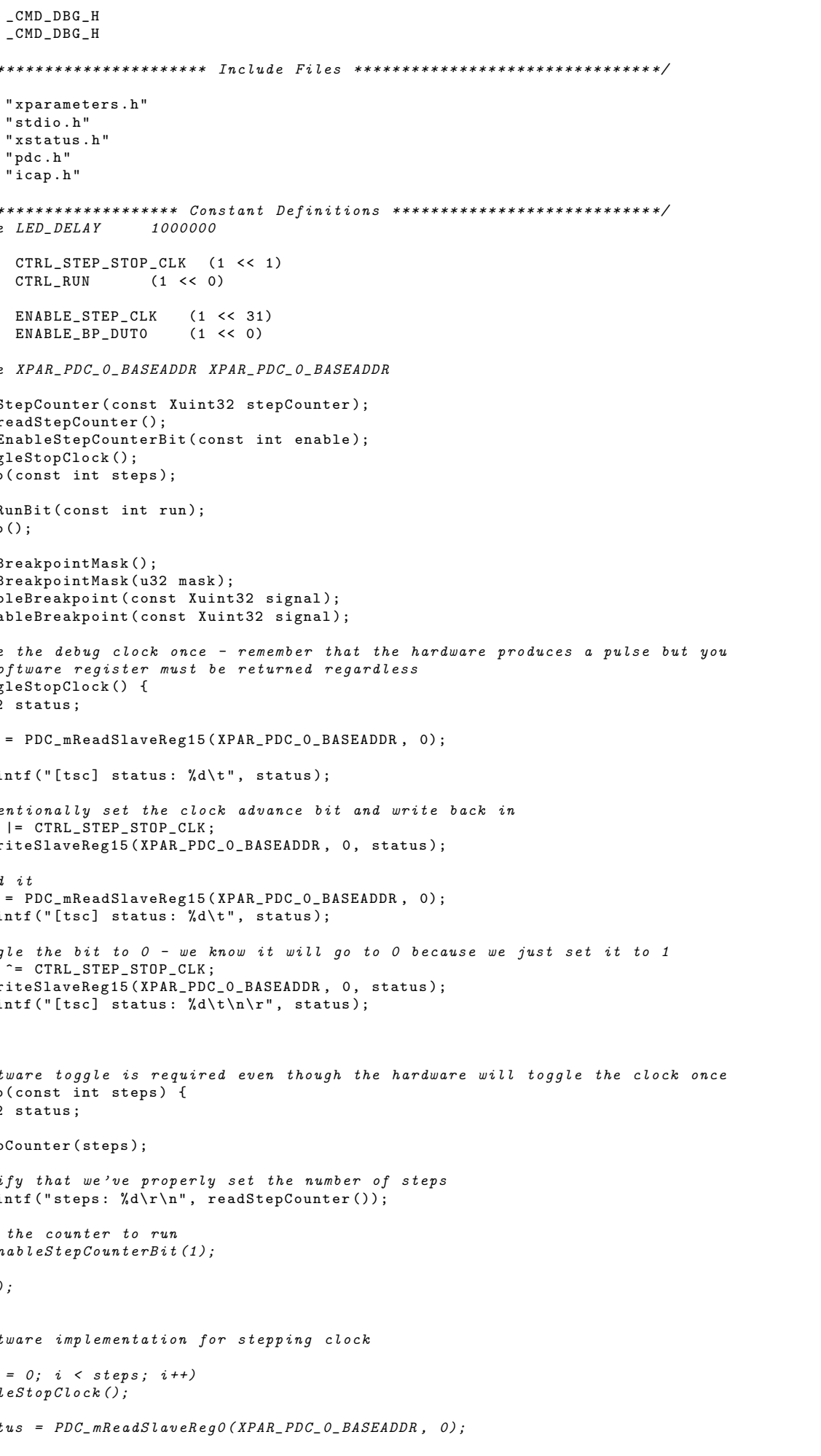




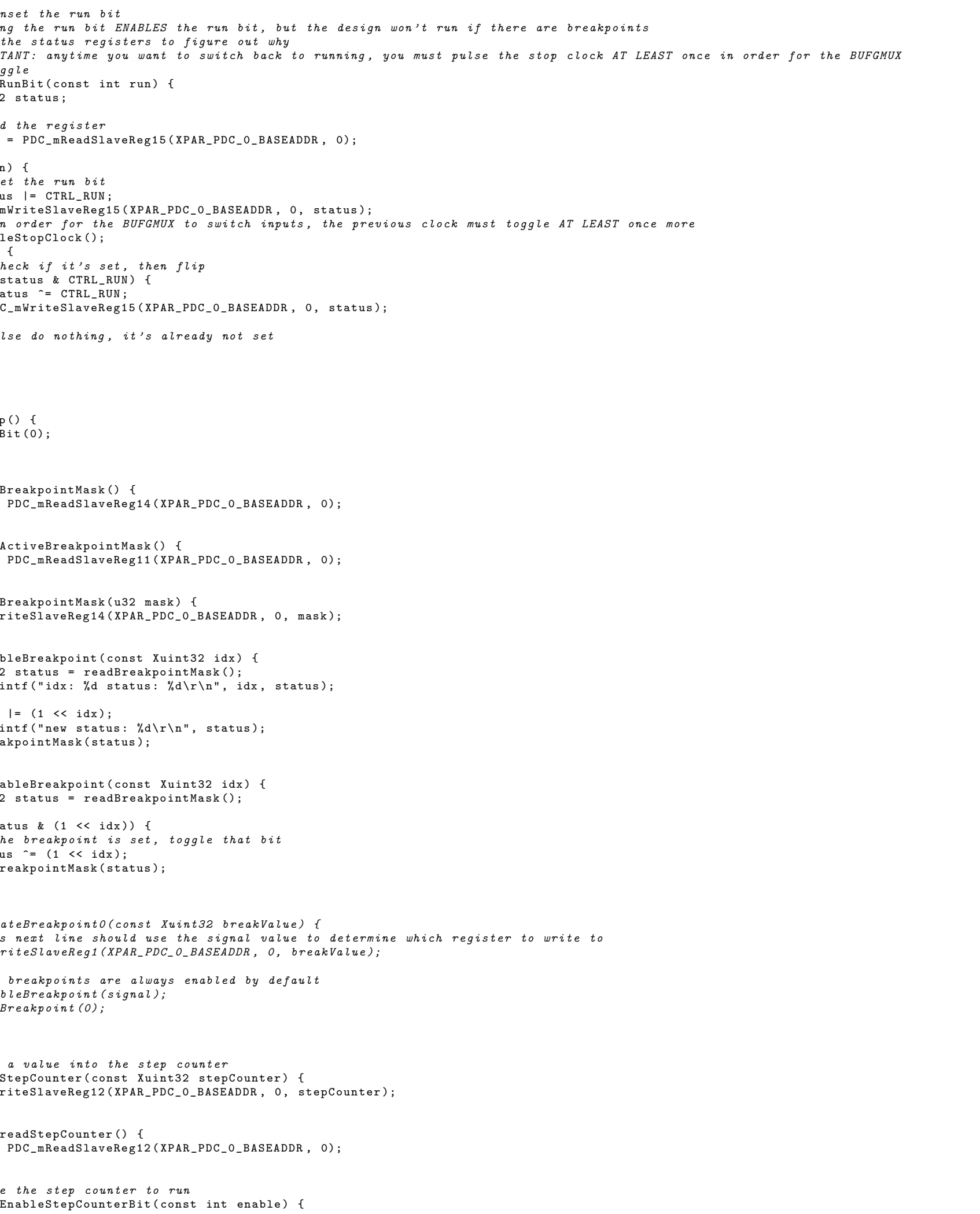




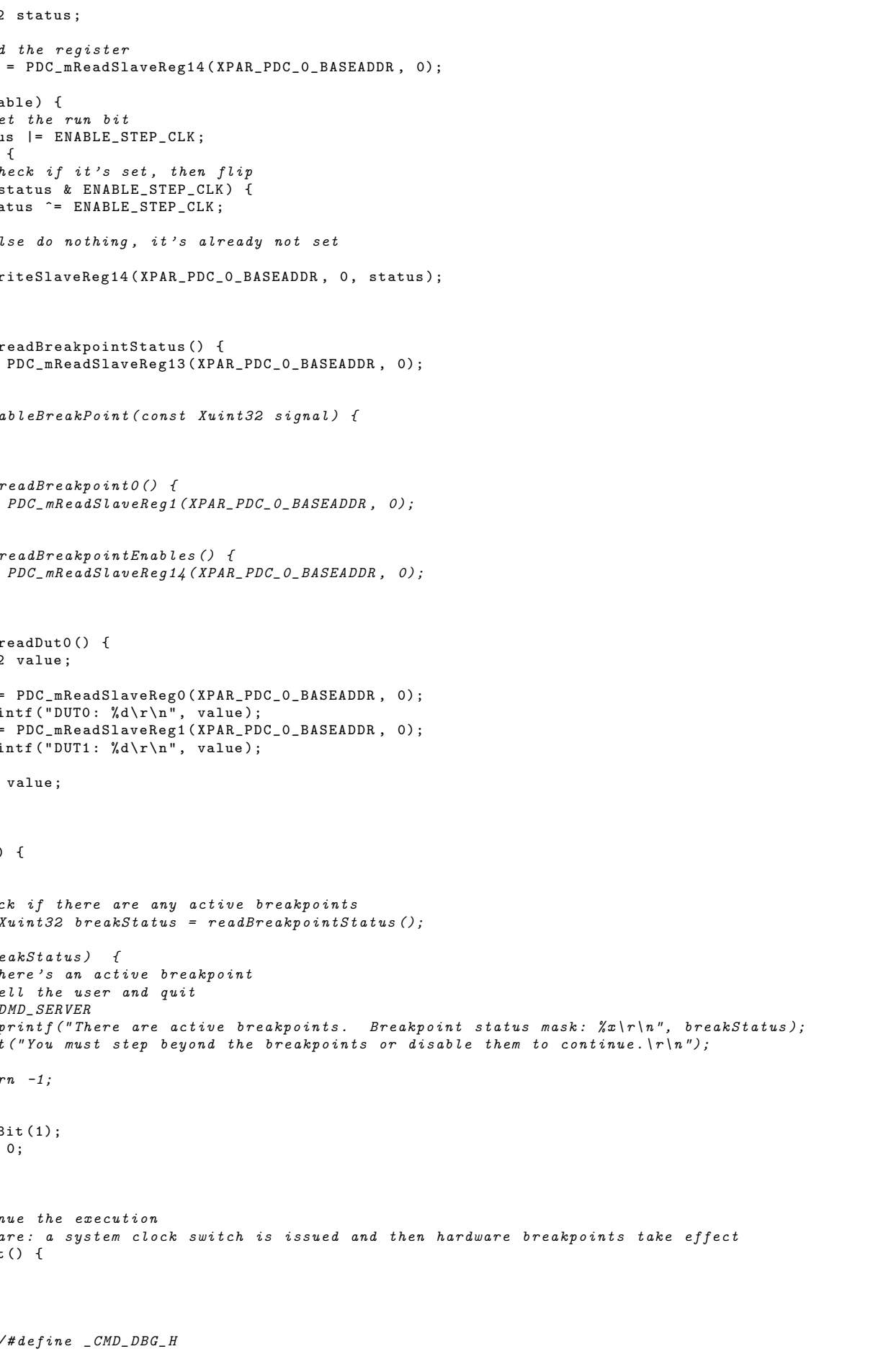


Listing B.8: ICAP Access Routines Header.

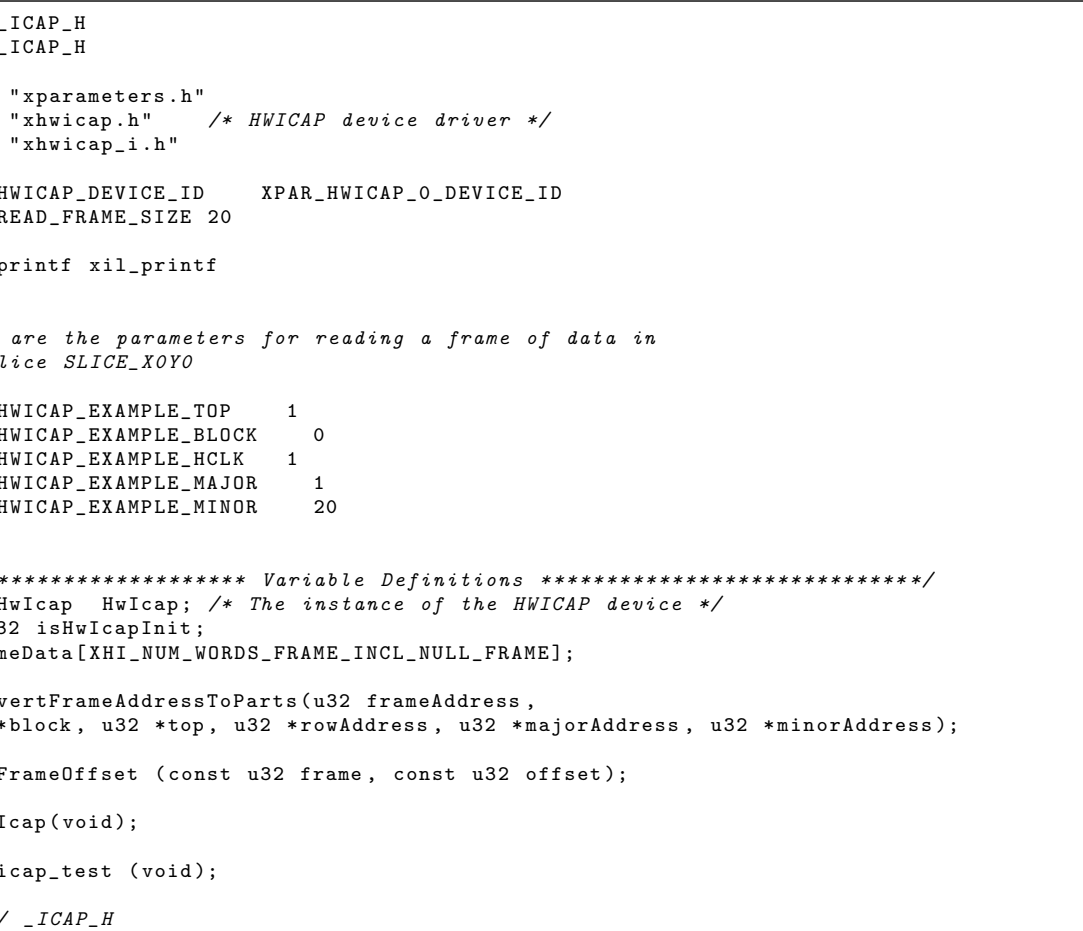


Listing B.9: ICAP Access Routines Source.

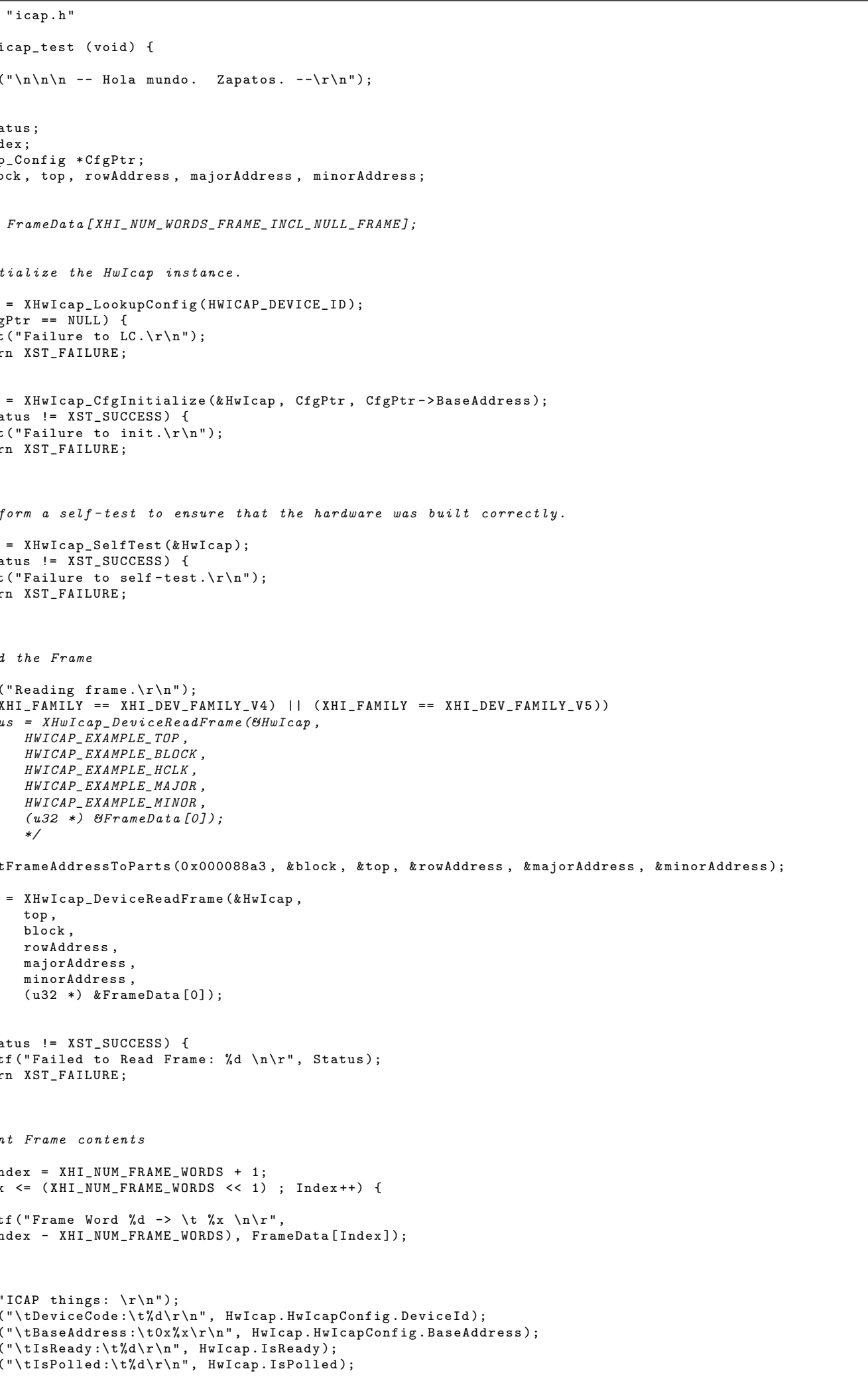




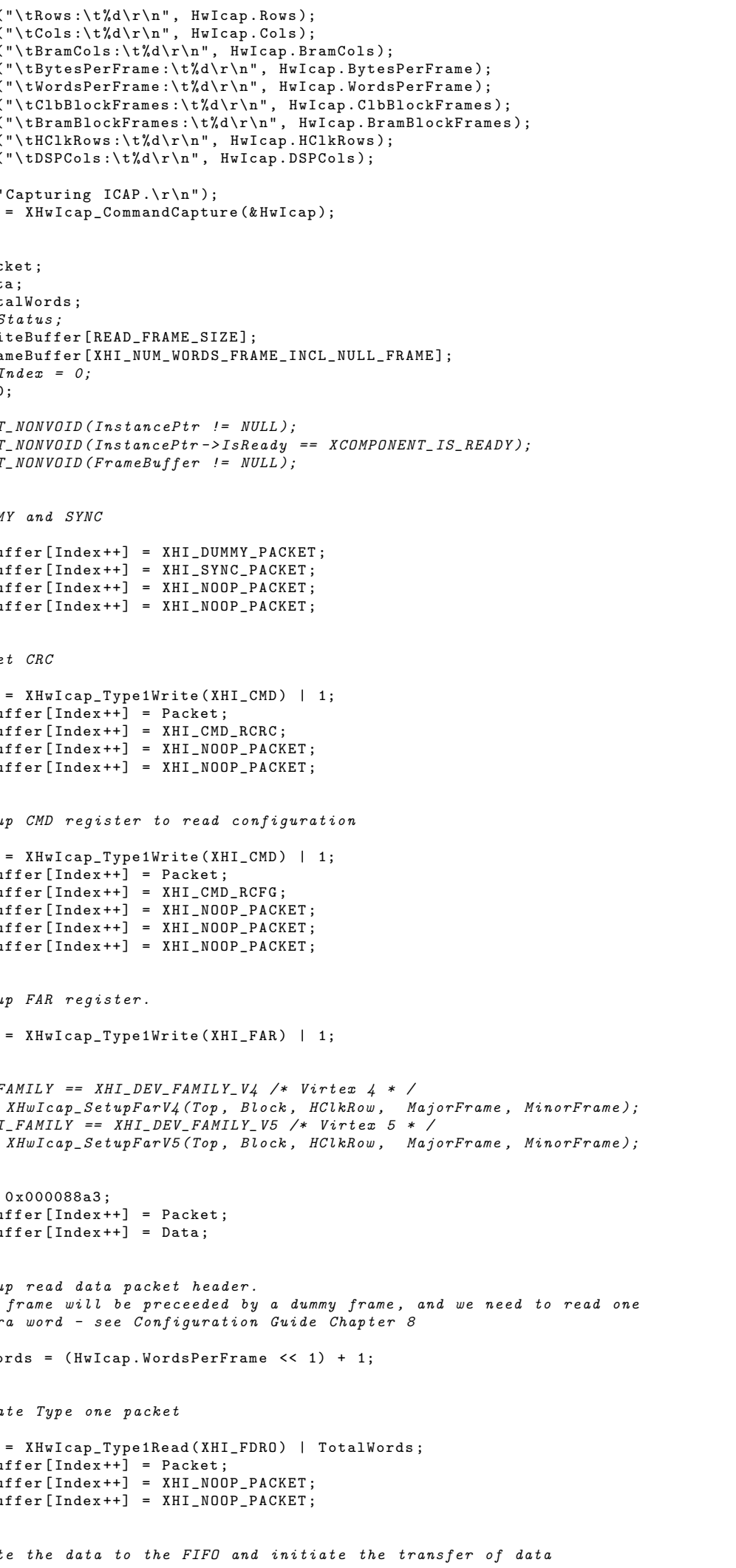




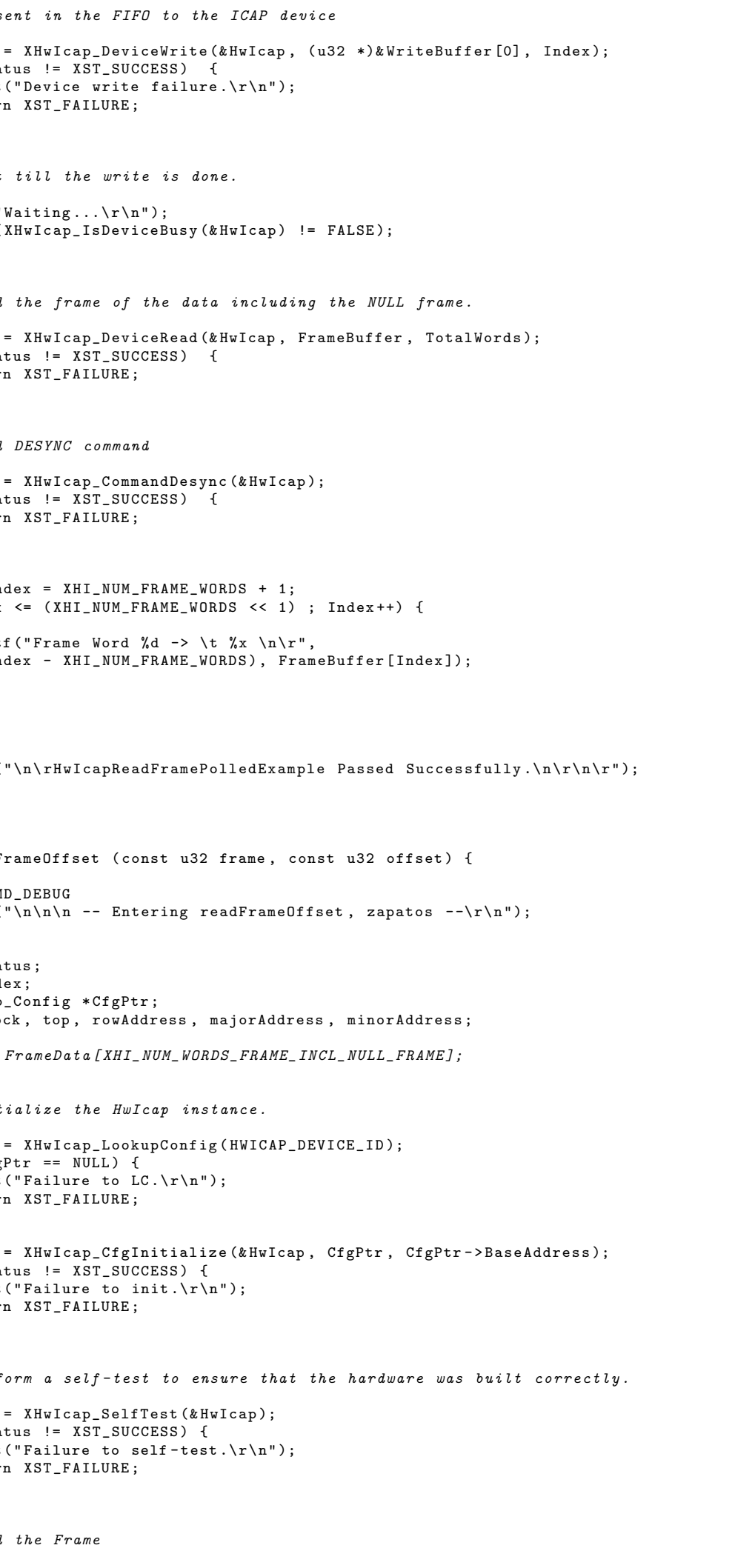




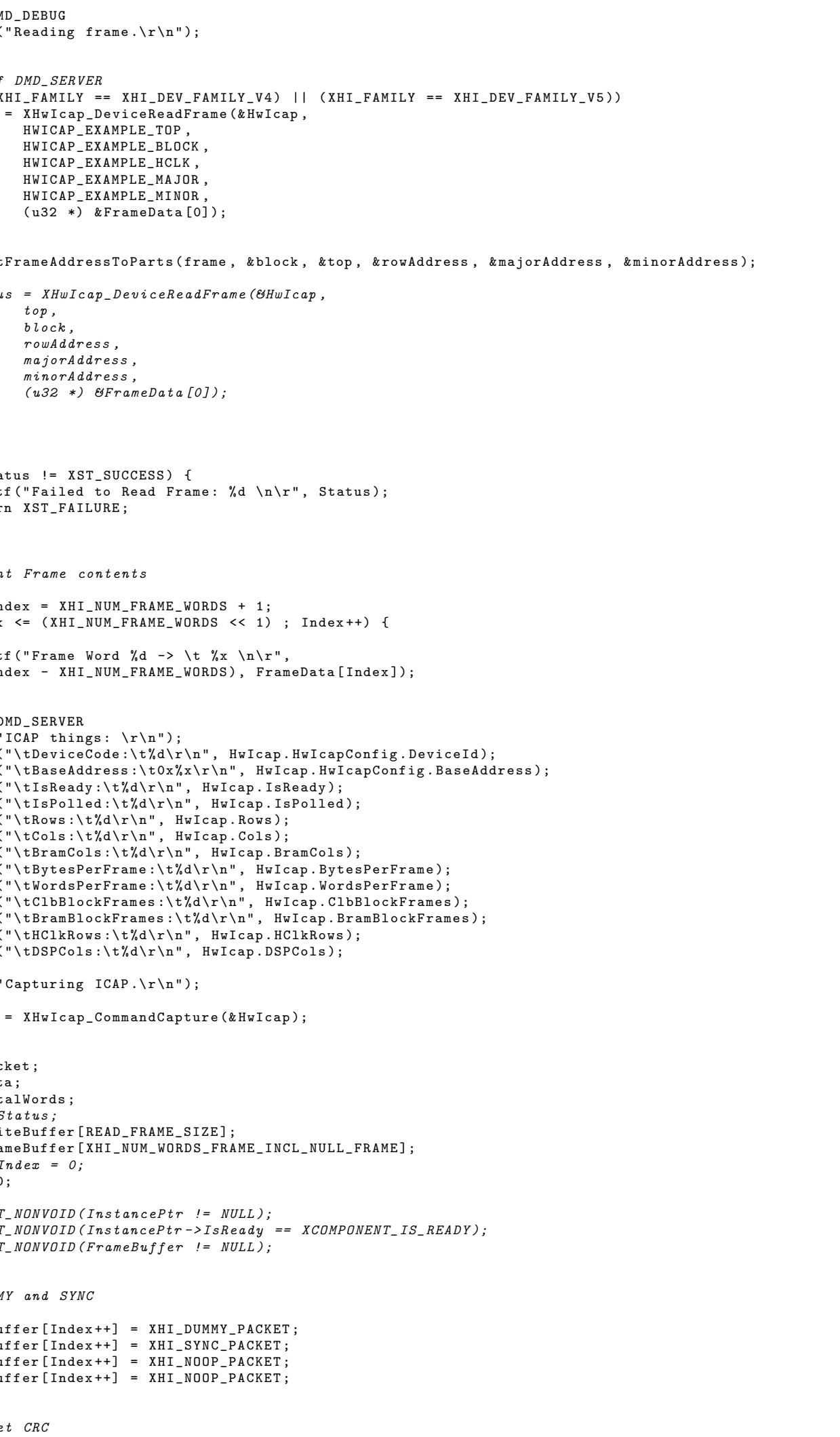




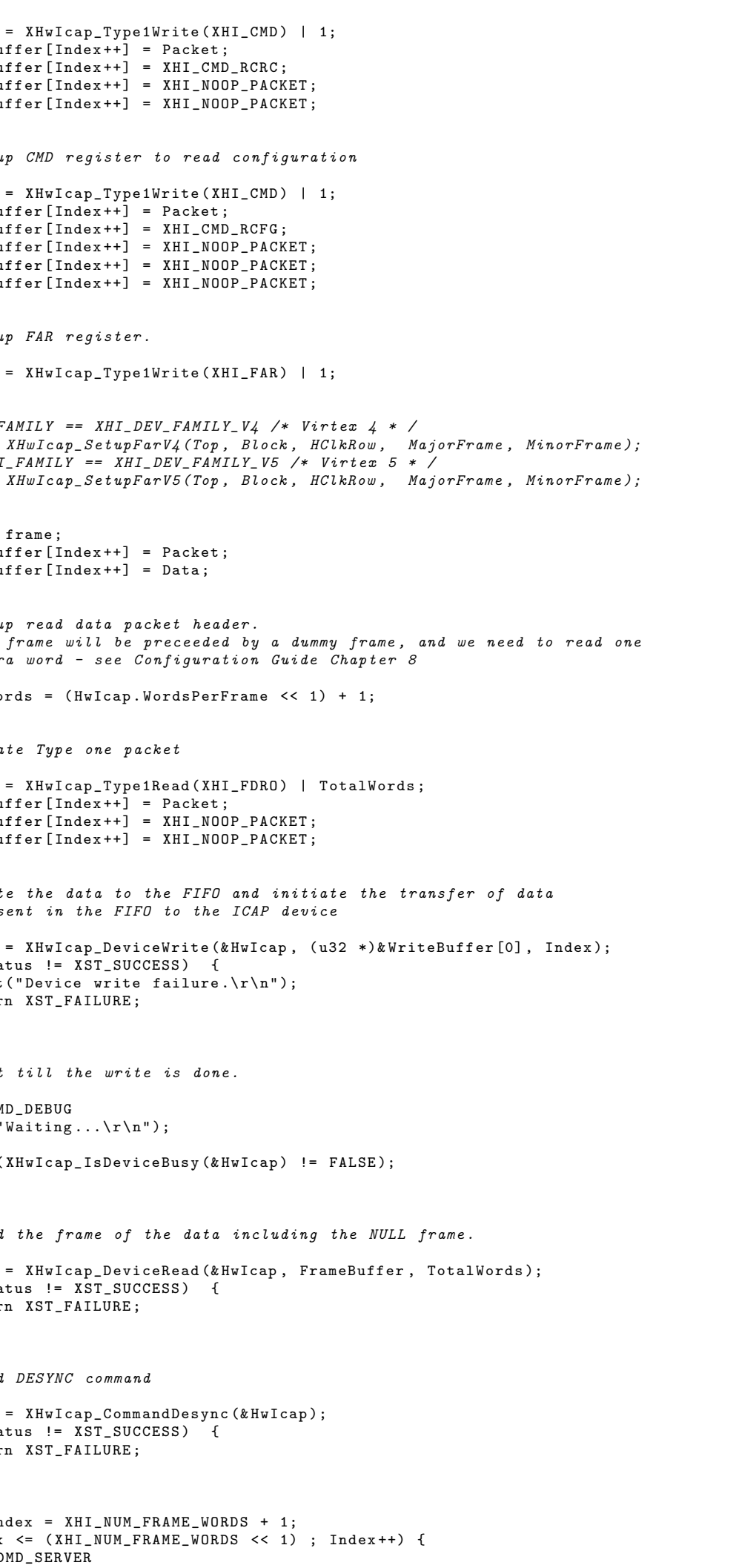




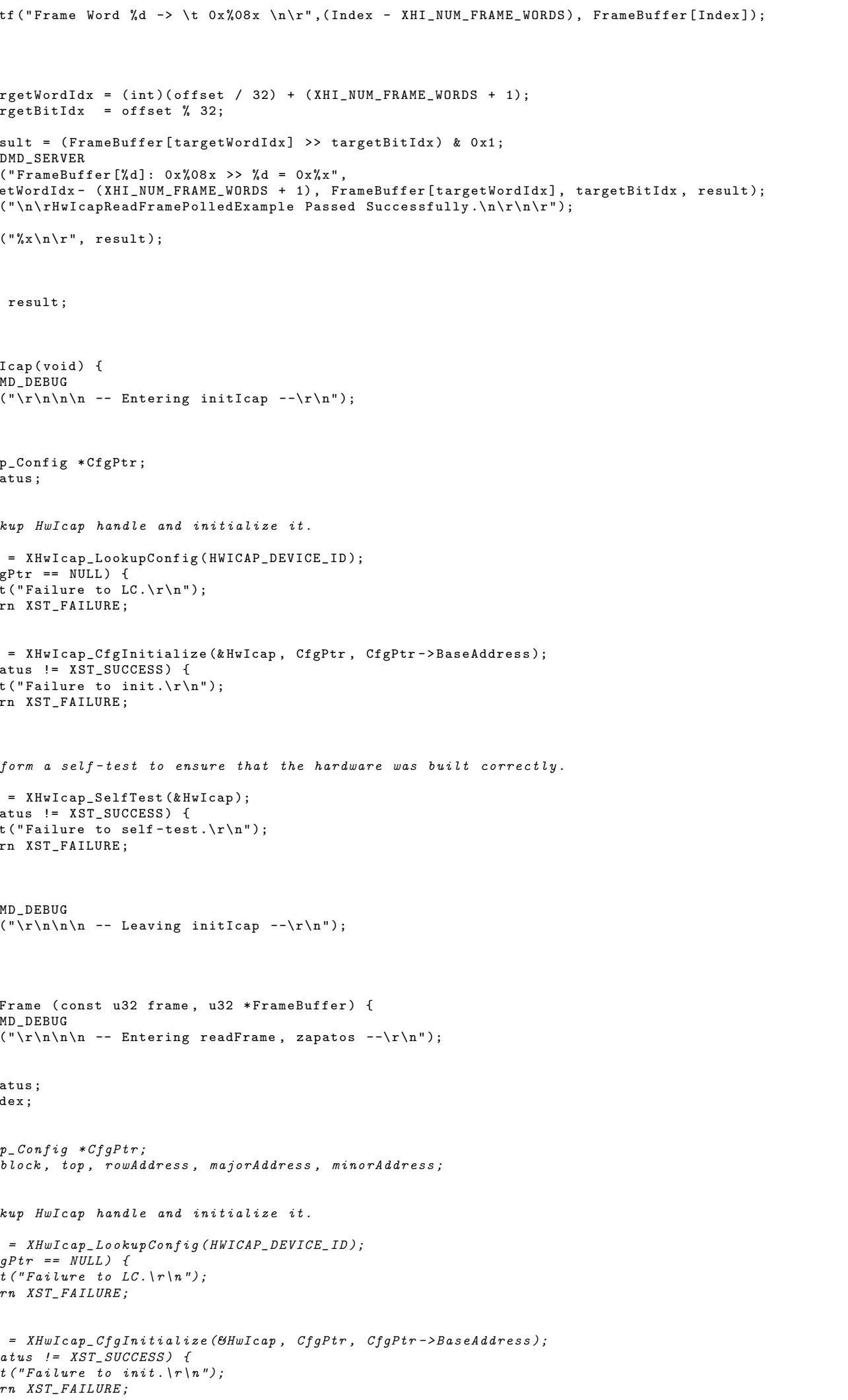




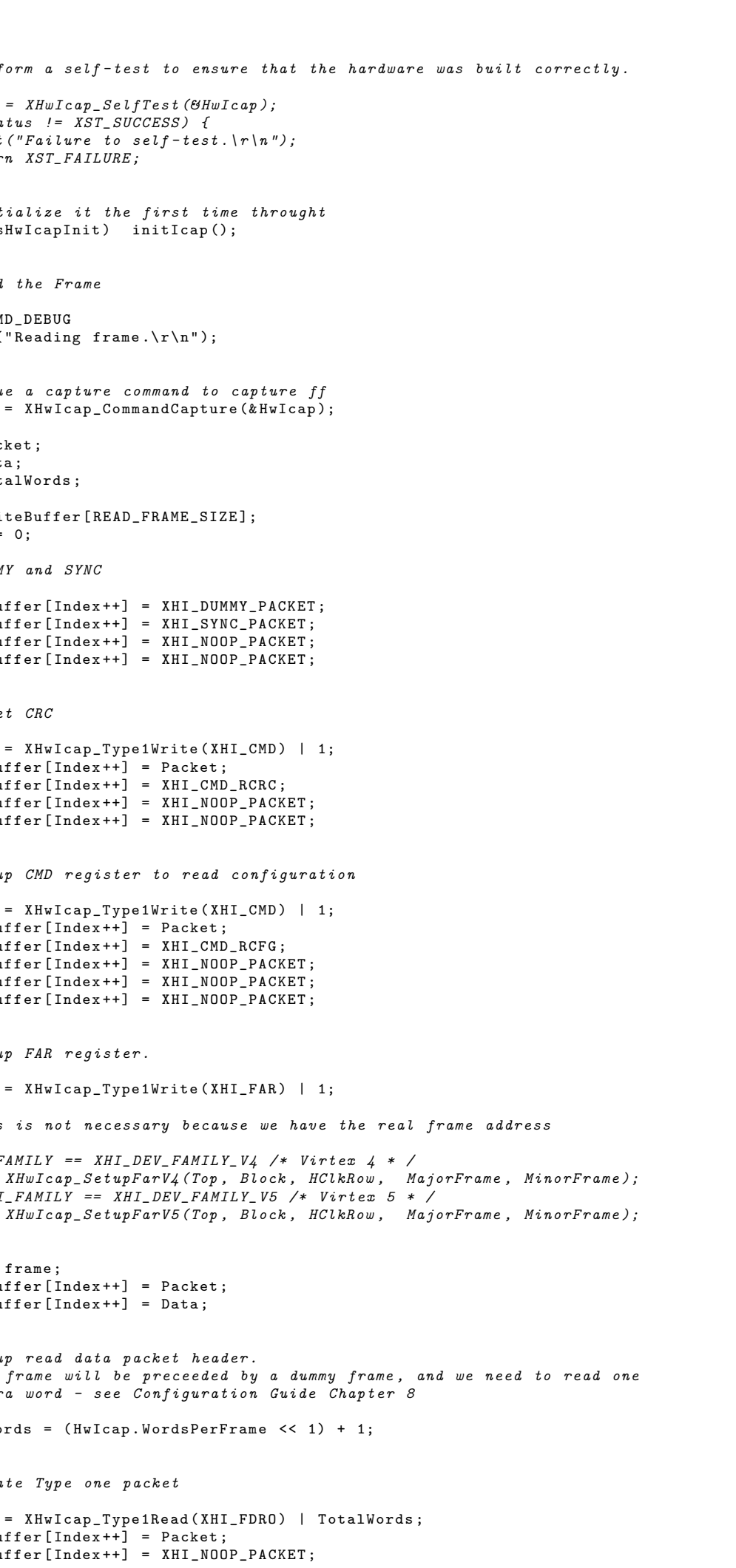




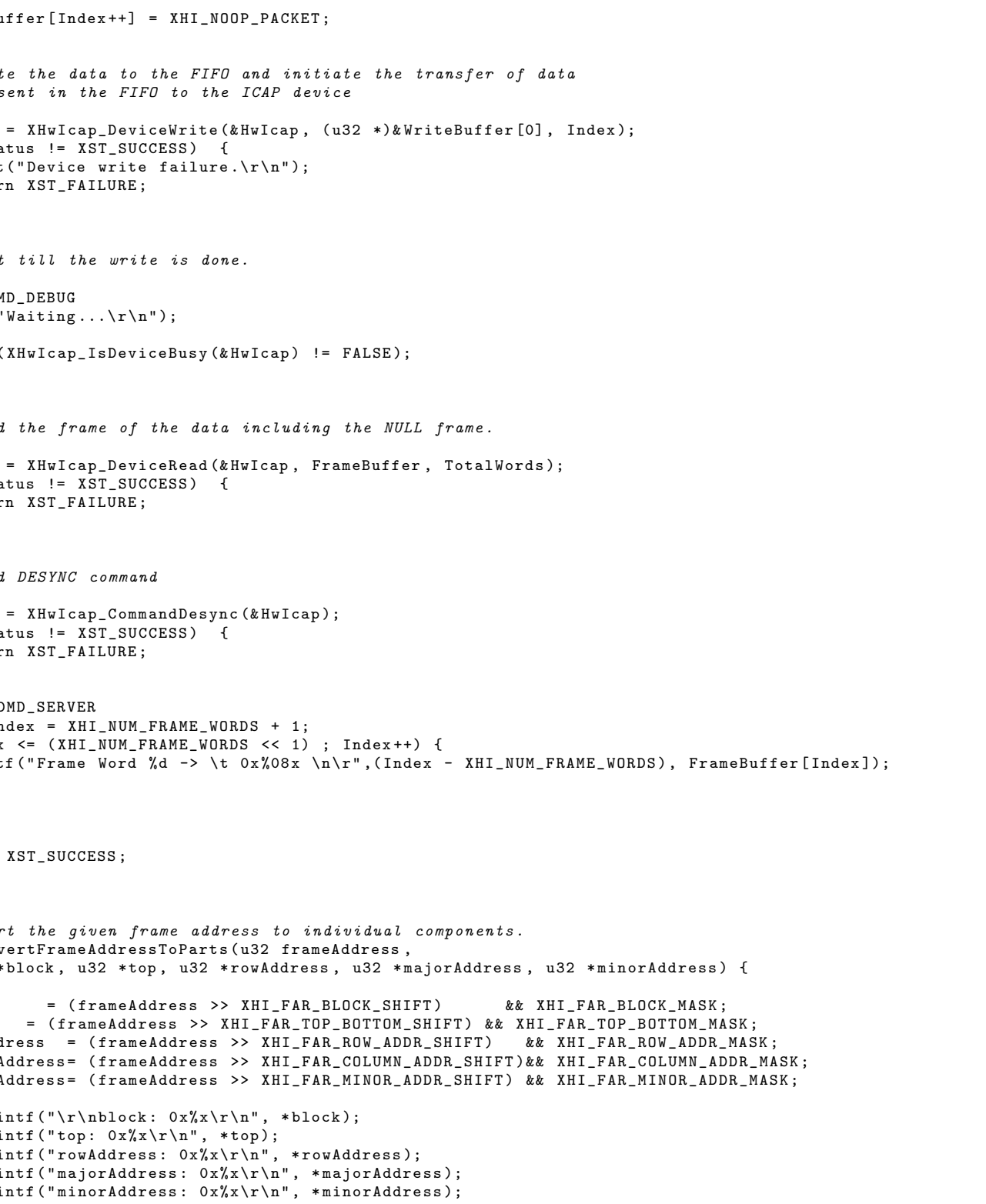




\section{Listing B.10: LLD Workstation Console Source.}

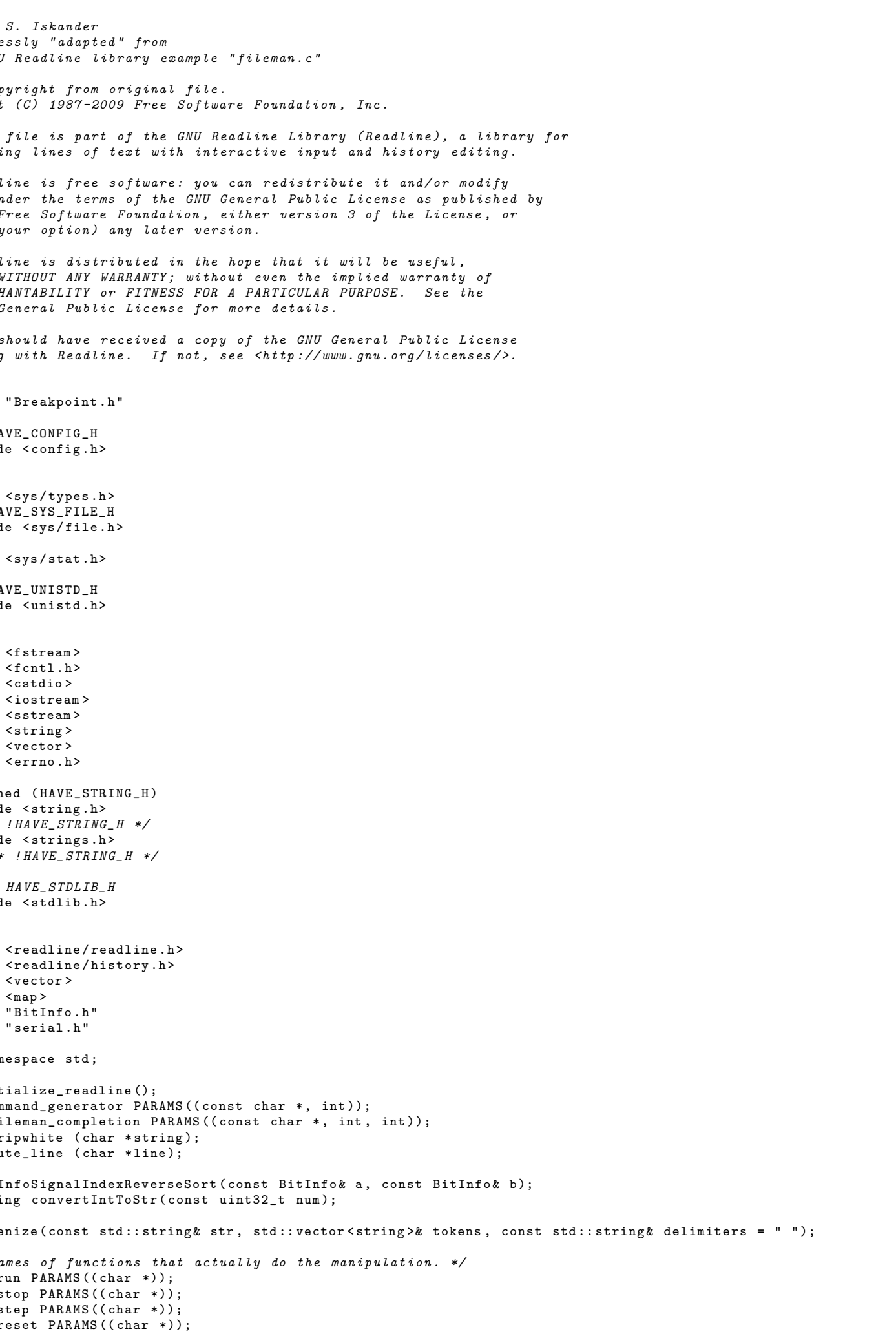




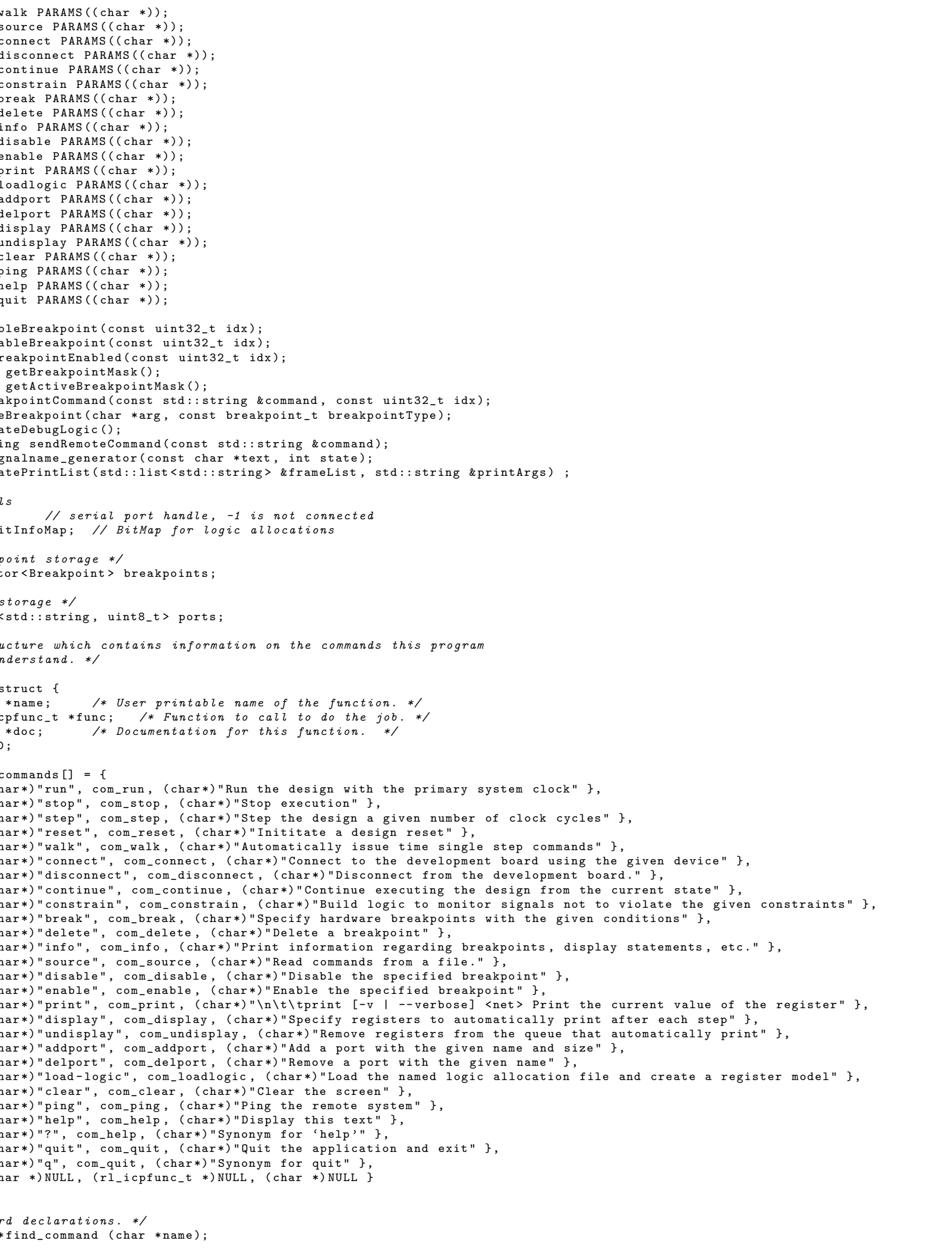




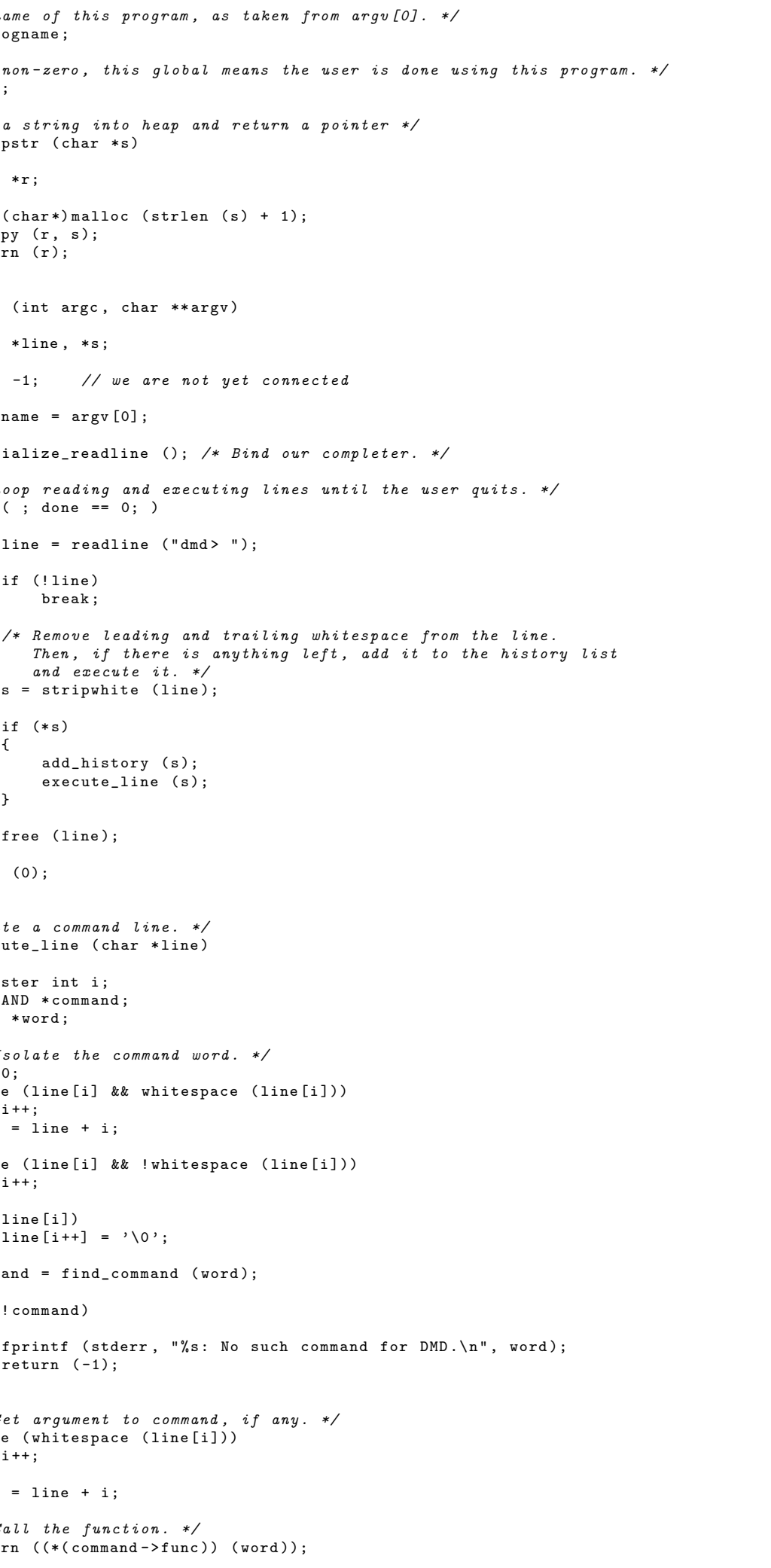




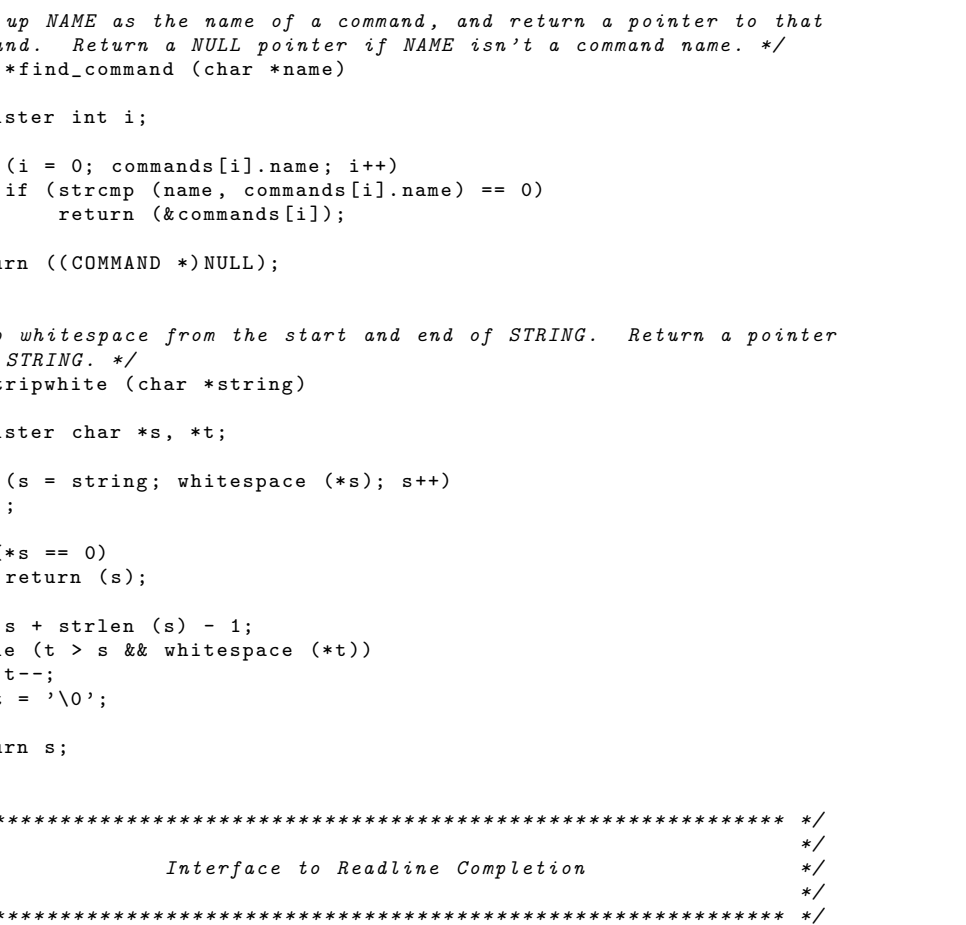




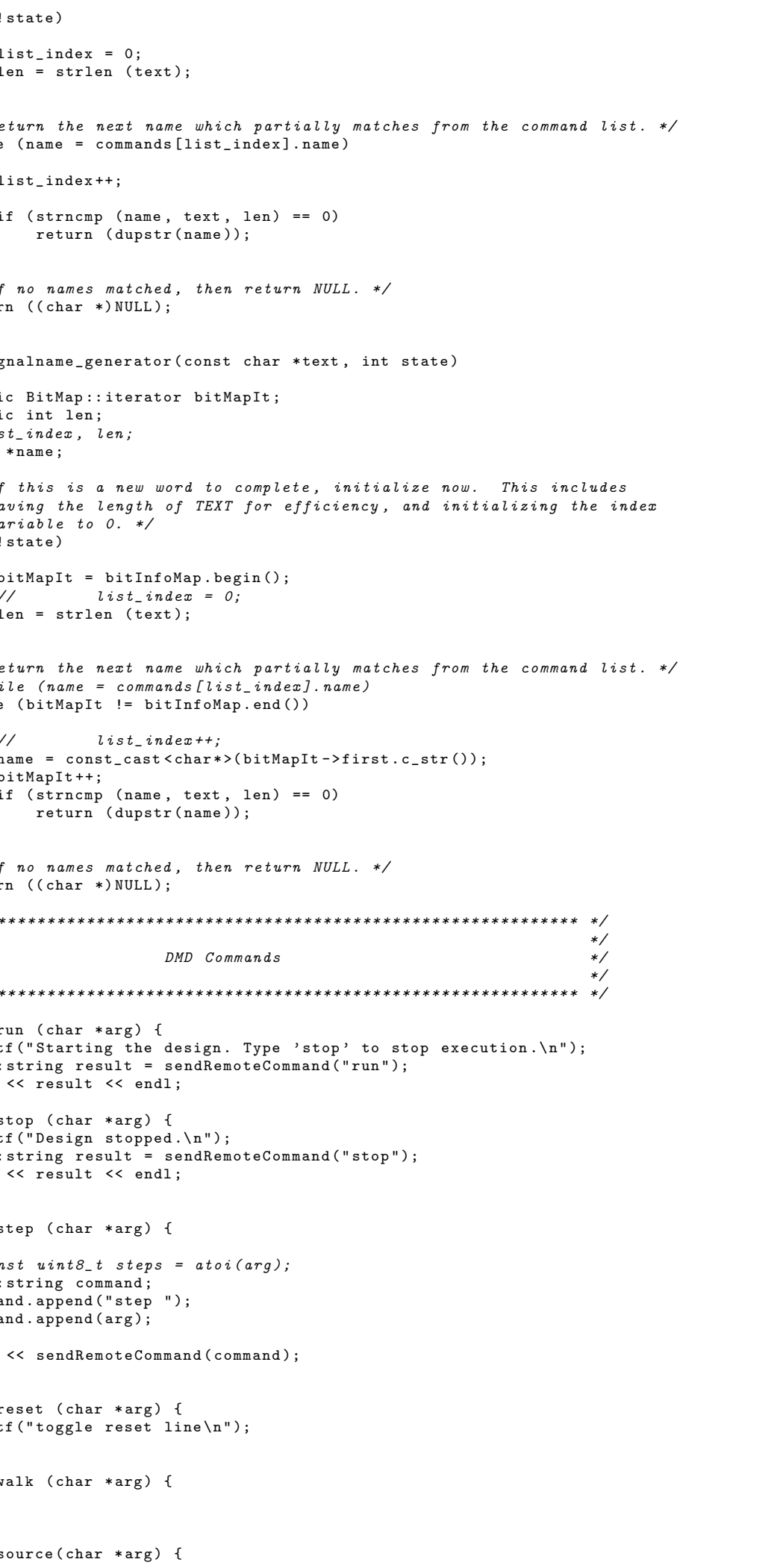




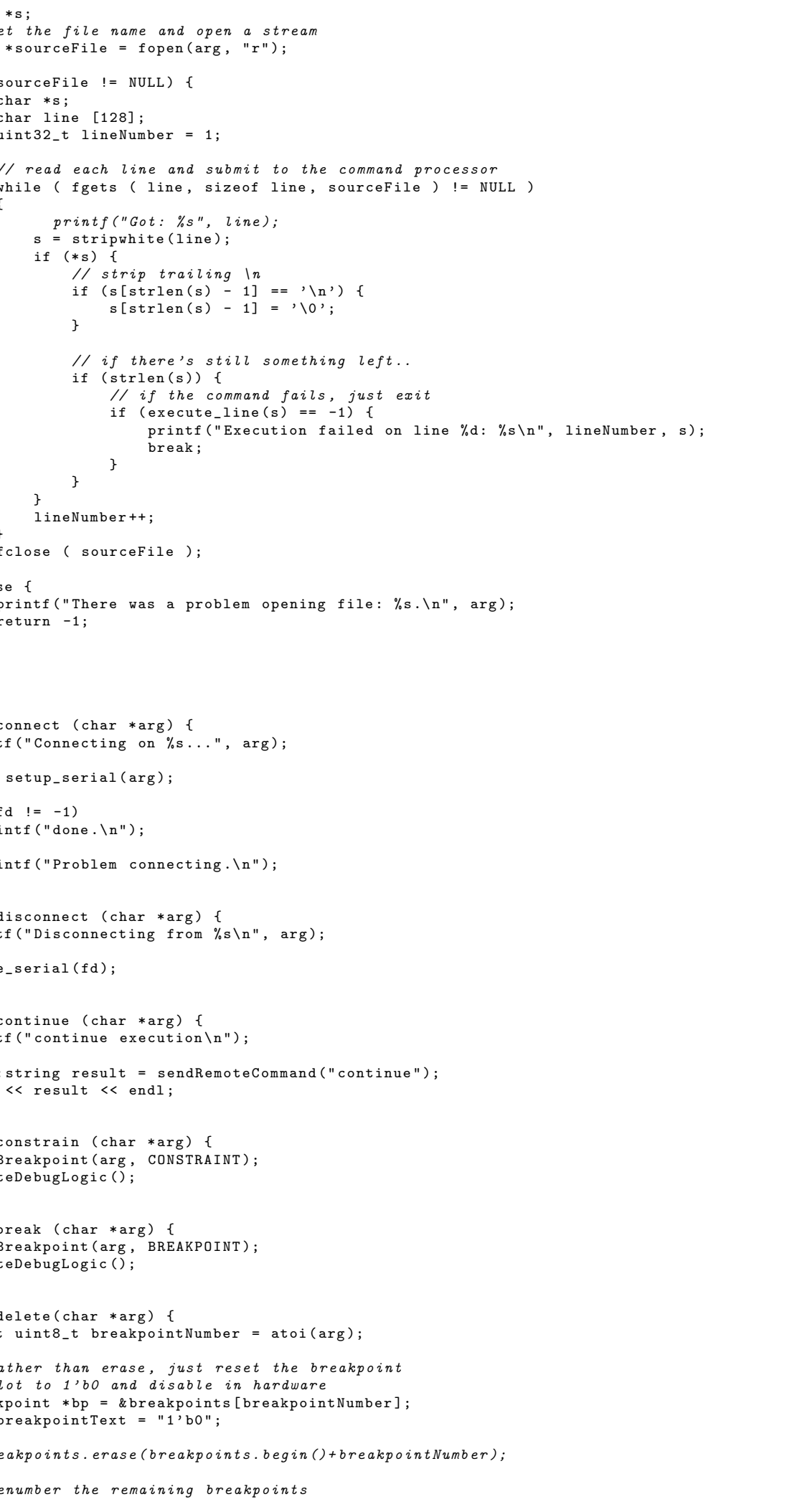




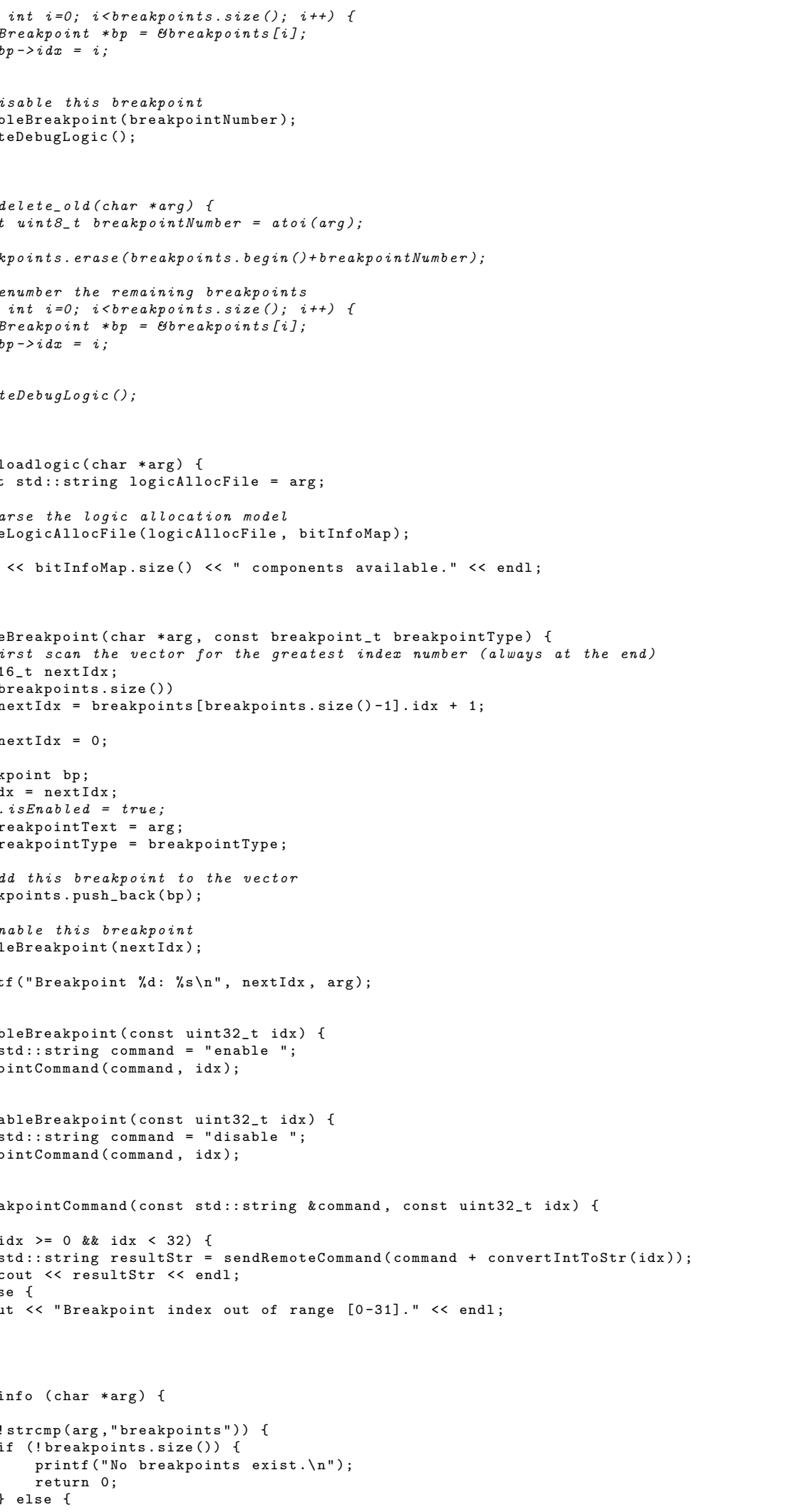




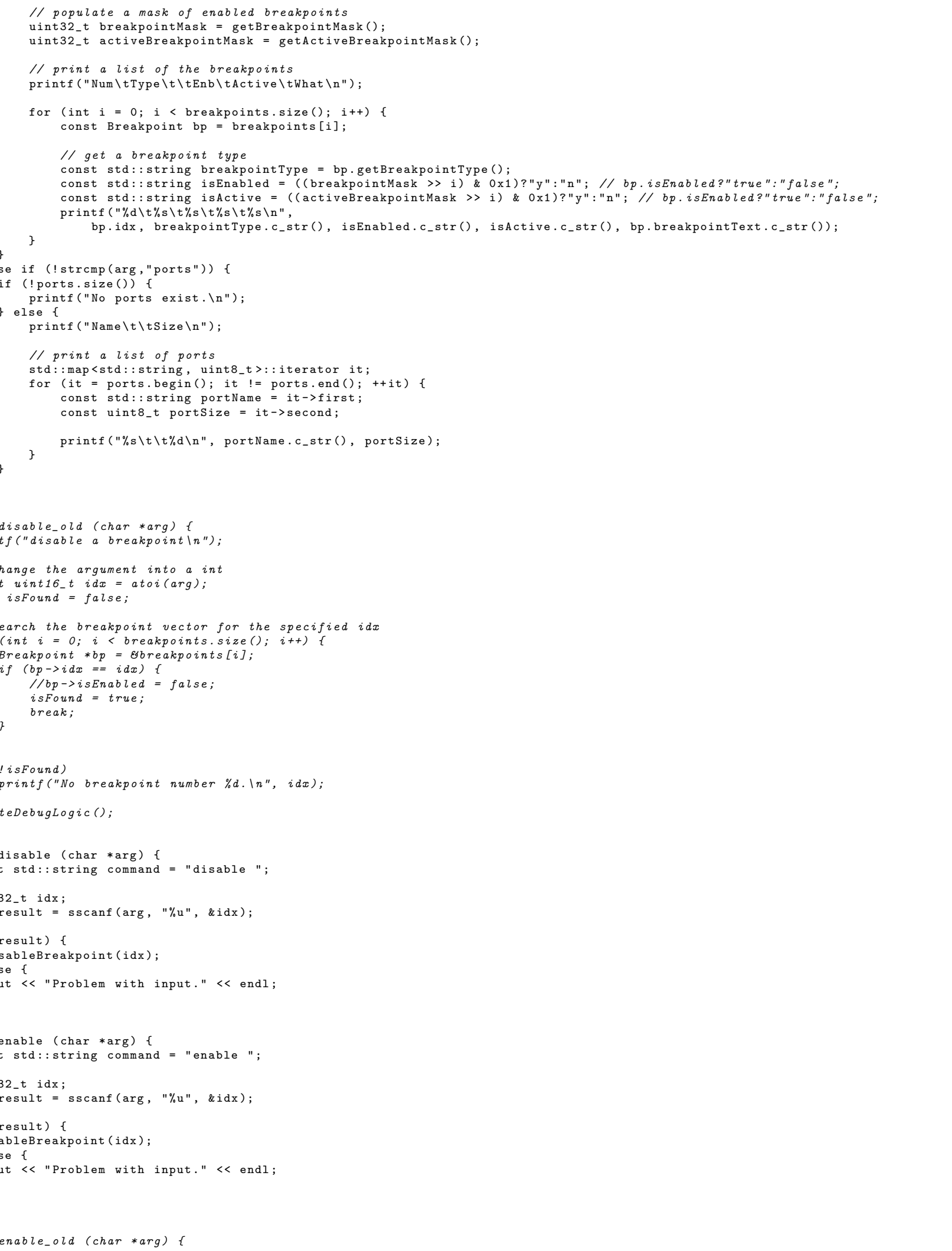




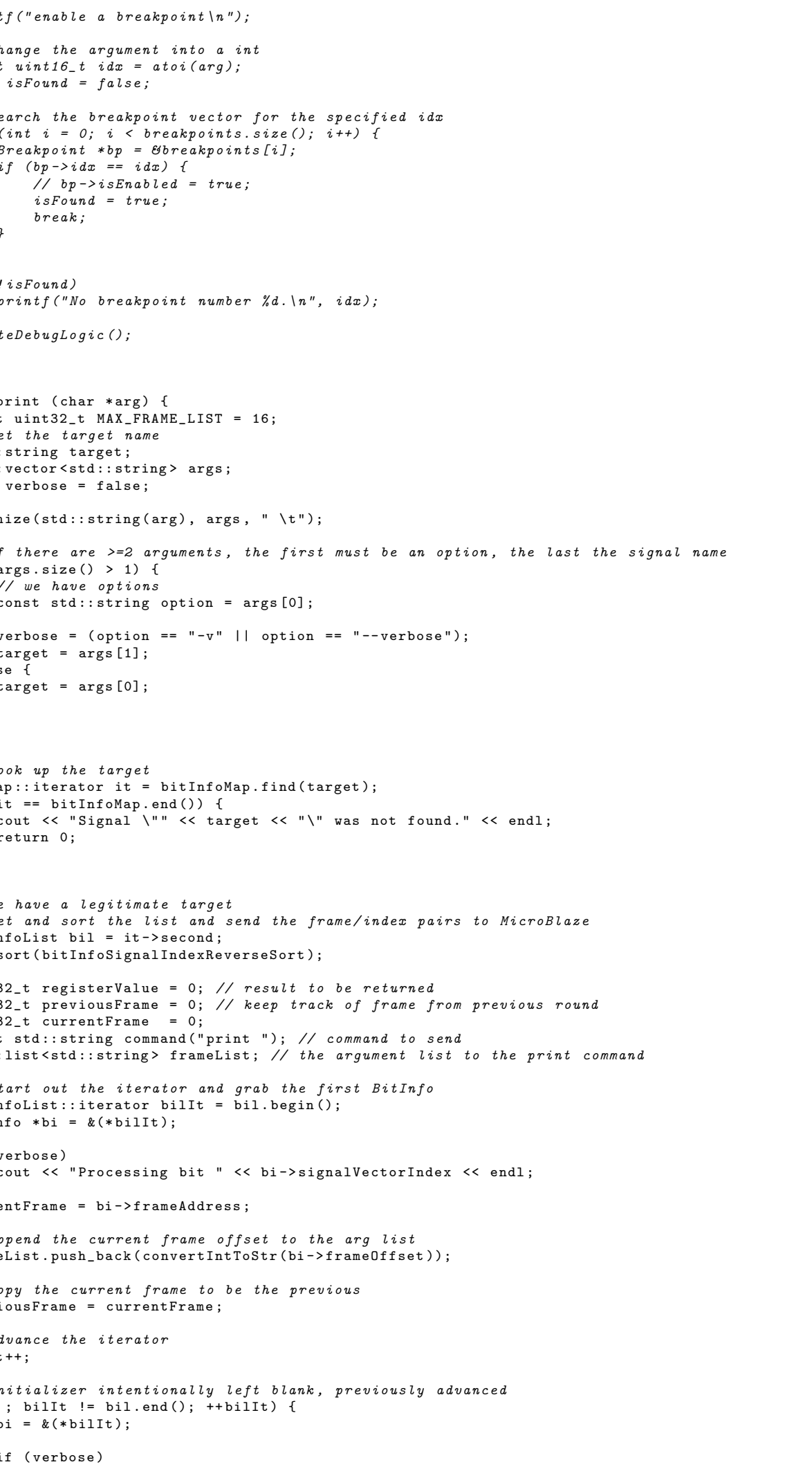


cout $\ll$ "Processing bit " $<$ bi->signalVectorIndex $\ll$ endl;

currentFrame $=$ bi $>$ frameAddress;

if ((currentFrame != previousFrame) || framelist.size () == MAX_FRAME LIST) f

// it's a new frame or the list is getting too big and it's time to empty

// send the pending command and add to the result...

// format the print command arguments, but first add the frame as the first argument

std: :string printArgs;

printArgs . append (convertIntToStr (previousFrame)) ;

createPrintList (frameList, printArgs);

if (verbose) \{

3

// process the result

std: : string resultStr = sendRemoteCommand (command + printArgs);

// read using sscanf because it's more tolerant of whitespace (atoi will error) uint32_t resultNum;

sscanf(resultstr.c_str(), "\%x", \&resultnum);

registervalue = (registervalue $<$ framelist.size()) | resultinu;

if (verbose)

printf("Response: $\% 5 \mathrm{~s} \% \# 08 x$ Current value: \%\#08x\n", resultStr.c_str(), resultNum, registerValue);

// clear the frameList...

framelist.clear ();

printargs.clear ();

\}

// append the frame offset as part of the current frame

framelist.push_back (convertIntToStr(bi->frame0ffset));

// issue the command and get the result, shift into register

I/ std: string result = sendRemoteCommand (command);

// cout $\ll$ command $\ll "->"<$ result $<$ endl;

// cout $\ll$ "Register $=" \ll$ registerValue $\ll$ endl;

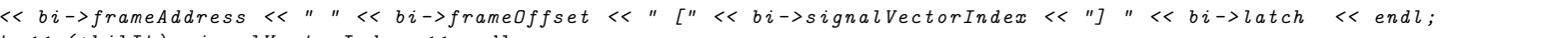
// cout $\ll(* b i l I t)$. signalVectorIndex $\ll$ endl.

previousFrame = currentFrame;

\}

// send the final command

// format the print command arguments

std: :string printArgs;

printArgs. append (convertIntToStr (previousFrame));

createPrintList (framelist, printargs);

if (verbose) \{ \}

cout $<$ "Sending remote command: $"<$ command $<" ~ "<$ printargs $<$ endl;

// process the result

std: : string result = sendRemoteCommand (command + printArgs);

$/ /$ cout $\ll$ endl $\ll$ command + printargs $\ll$ endl;

I/ cout $\ll$ "regValue (before final) = " $<$ hex $\ll$ registerValue $\ll$ "frameList.size() = " $\ll$ dec $\ll$ frameList.size() $\ll$ endl; I/ cout $\ll$ "result (str)," $<$ result $\ll "$ result (atoi) " $<$ atoi(result.c str()) $\ll$ endl;

//cout $\ll " R e s u l t=$, $<$ result $\ll "$ registerVal = " $<$ registerValue $\ll$ endl;

uint32_t resultNum;

sscanf (result.c_str(), "\%x", \&resultnum);

registerValue $=$ (registerValue $<$ framelist.size () ) | resultNum;

if (verbose)

printf("Response: $\% 5$ s \% $\# 08 x$ Current value: $\% \# 08 x \backslash n "$, result.c_str(), resultNum, registerValue);

// the result must be inverted first before being used

// however we did this on the MBlz because short vectors were being corrupted

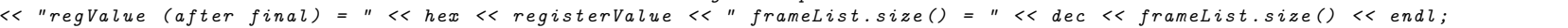

// registerValue $=$ registerValue

printf ( $\% \# 8 x$ (unsigned) $\%$ un", registerValue, registerValue); 


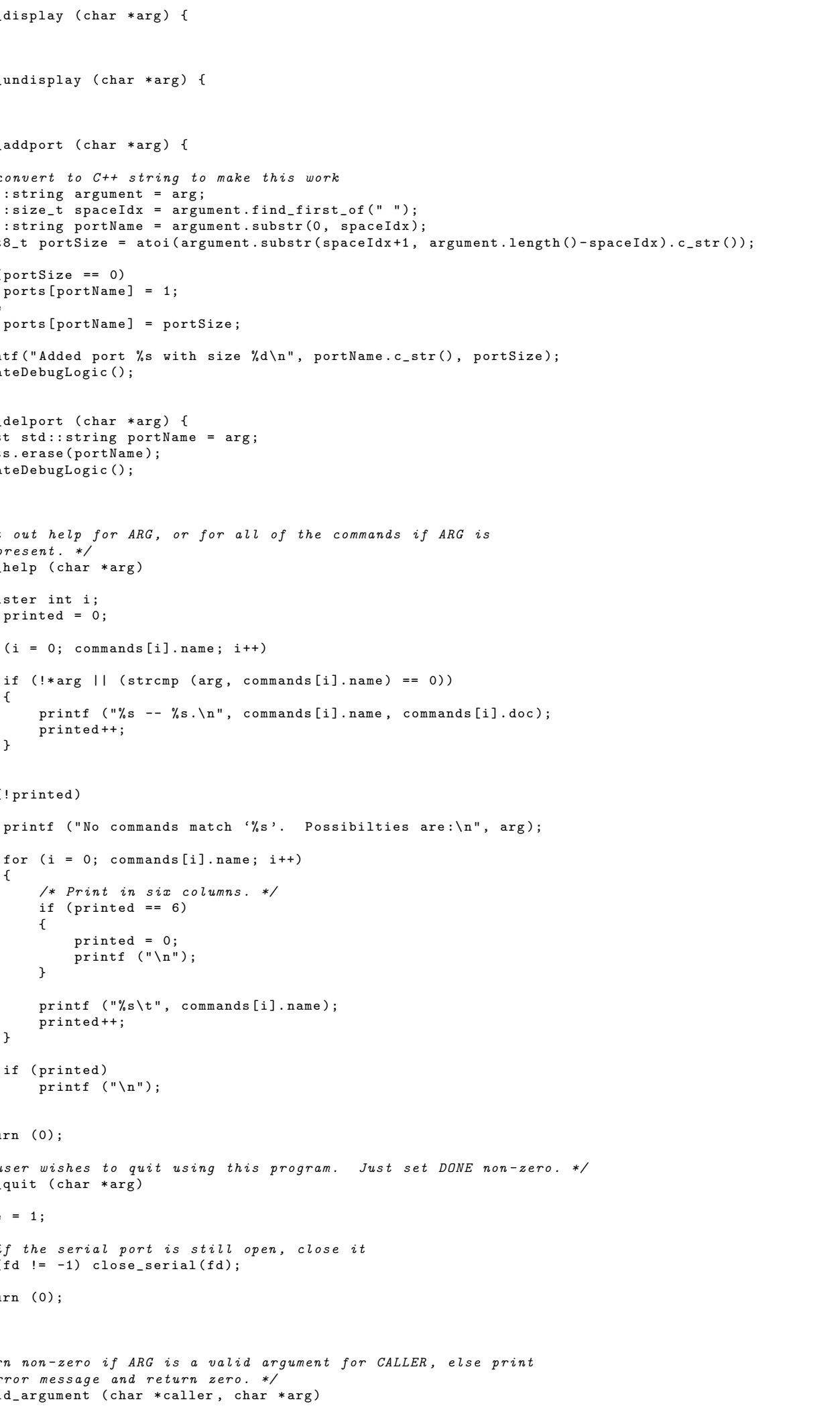




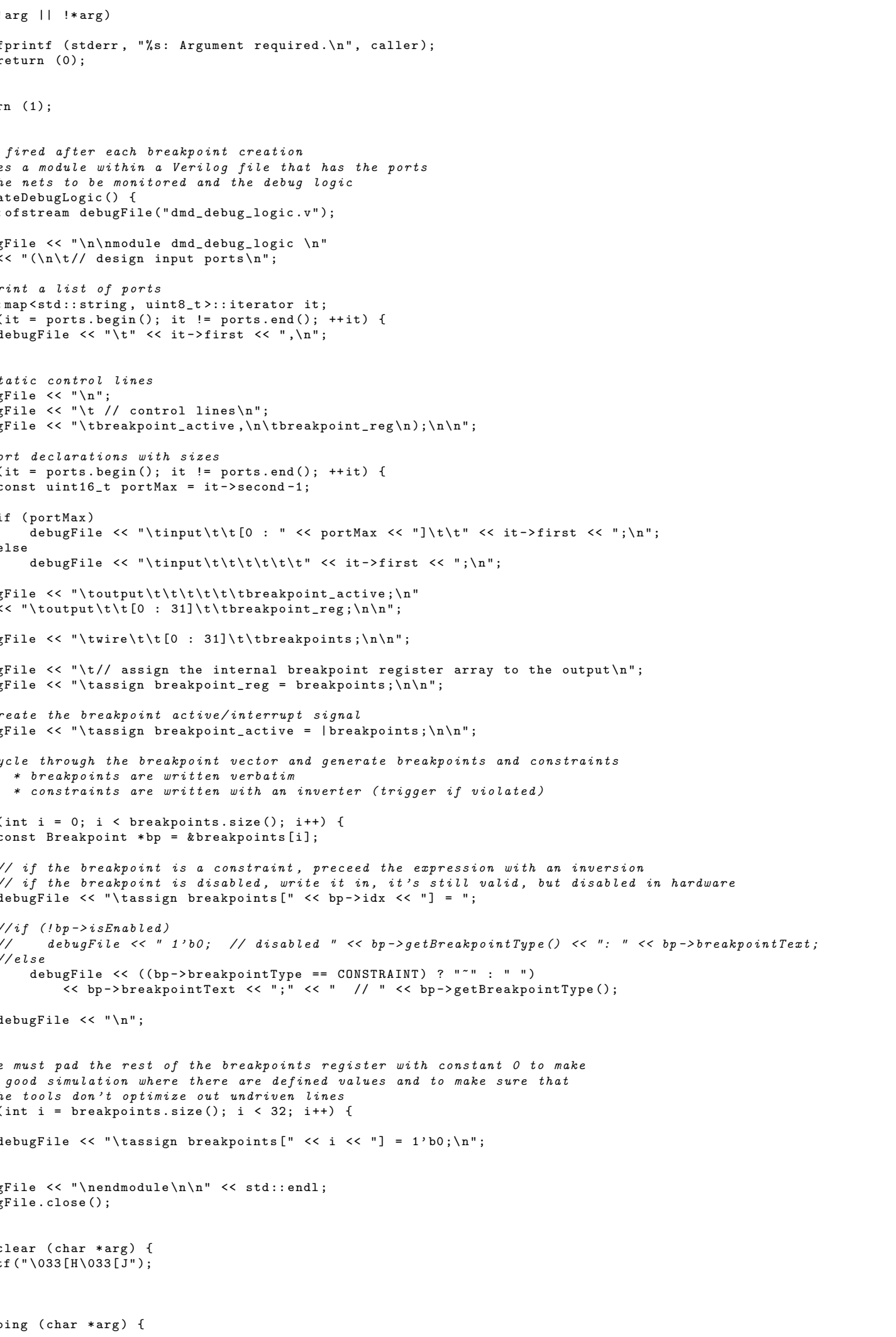




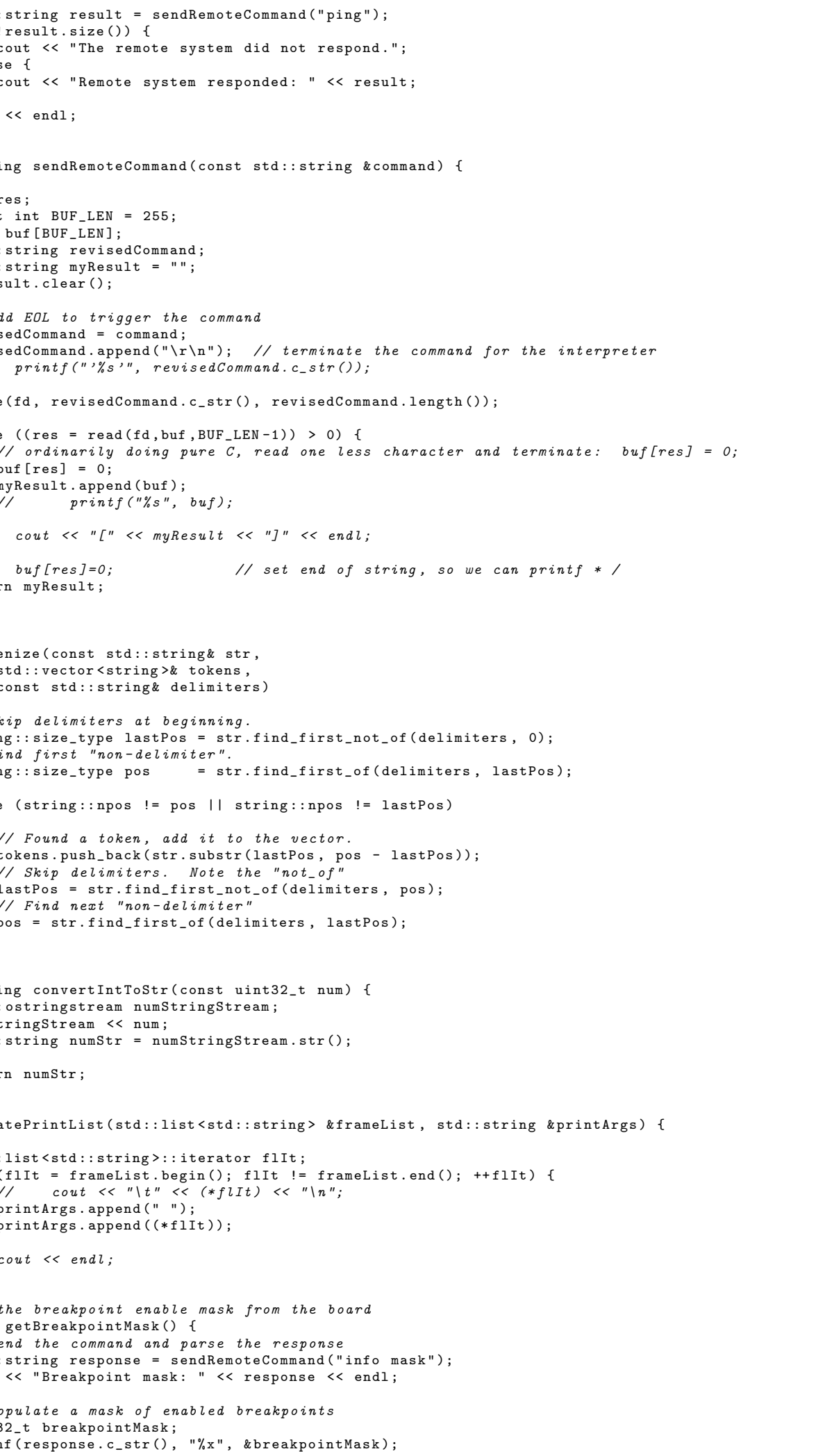


Yousef S. Iskander

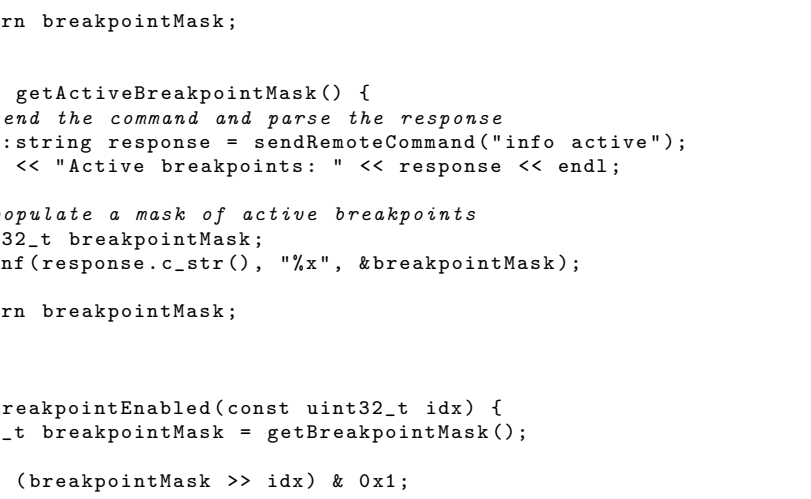


Listing B.11: Main FPGA LLD Console.

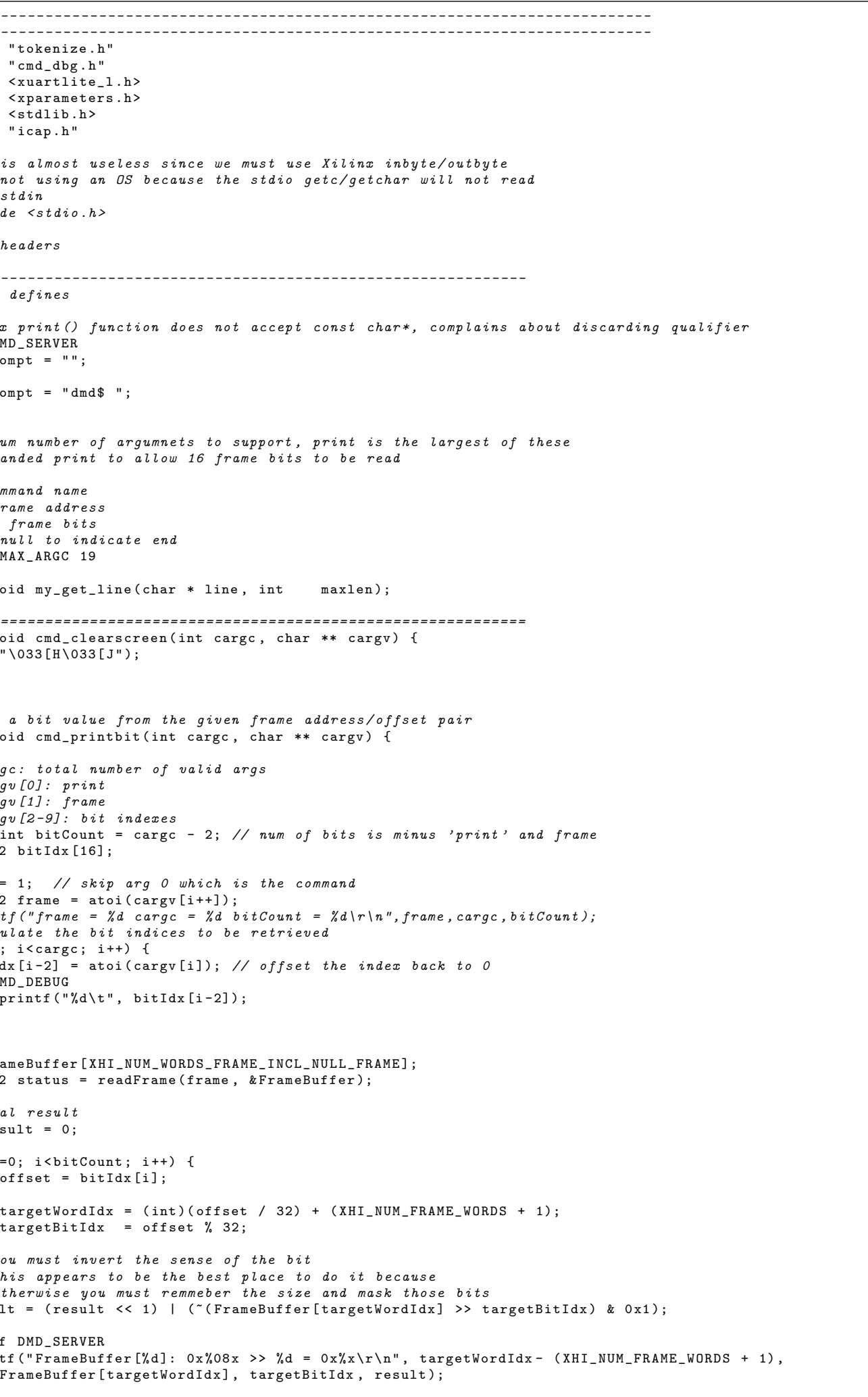




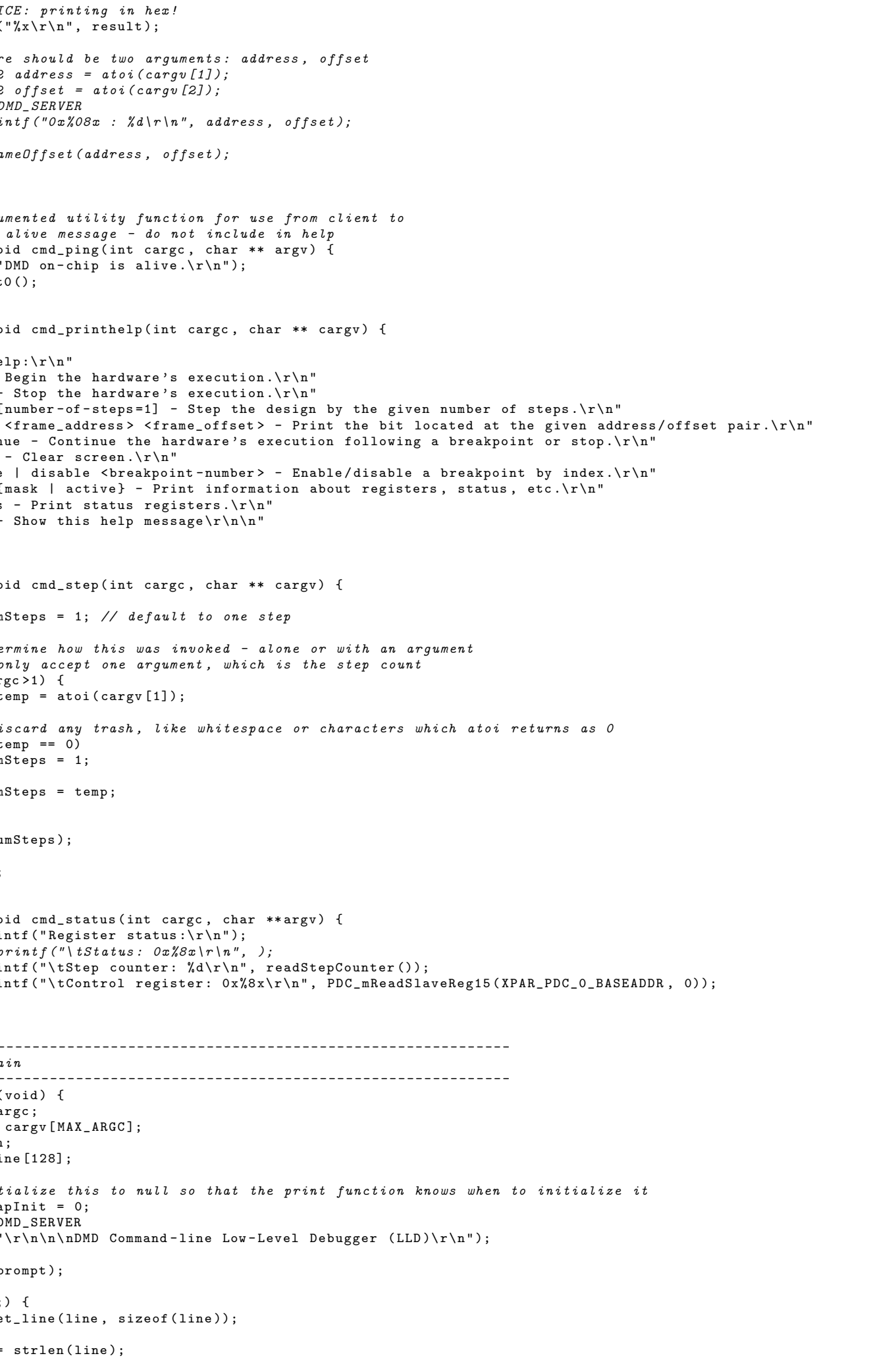




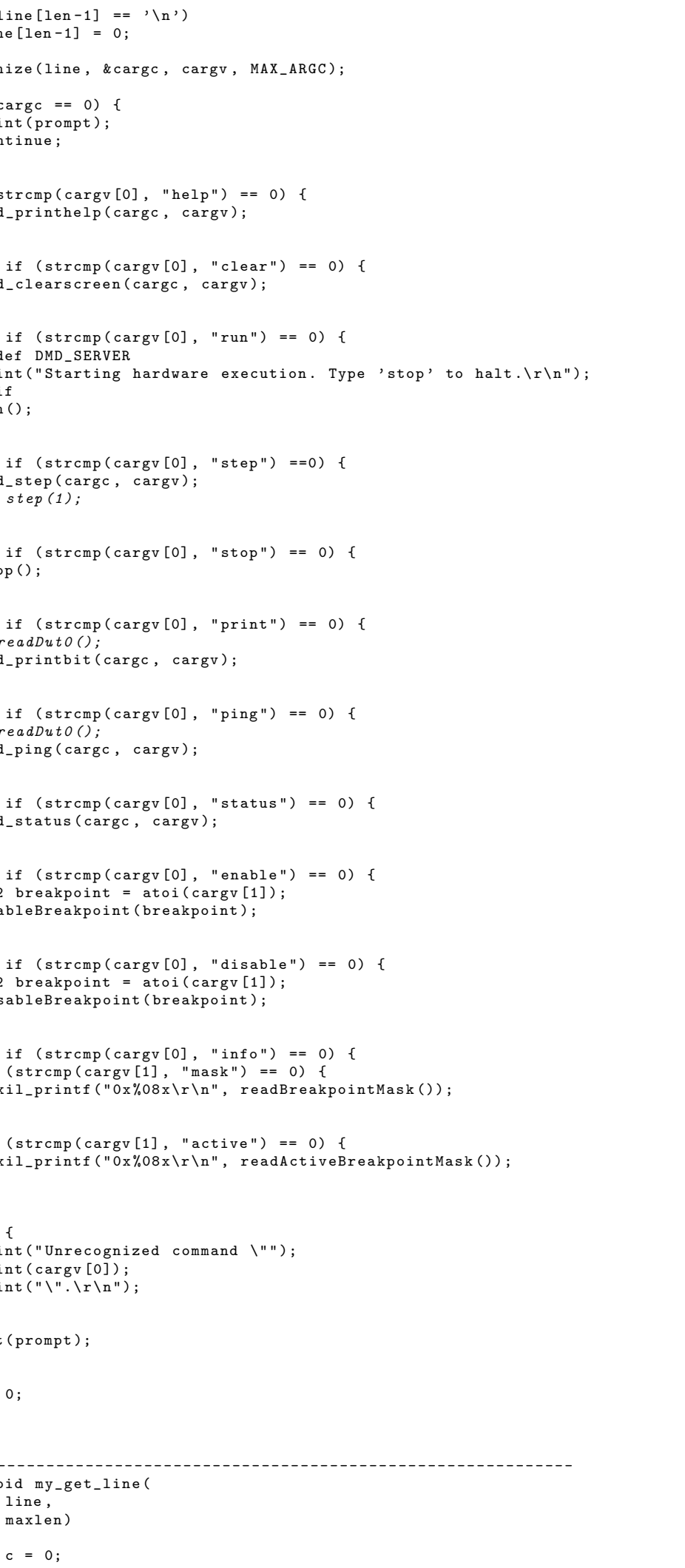




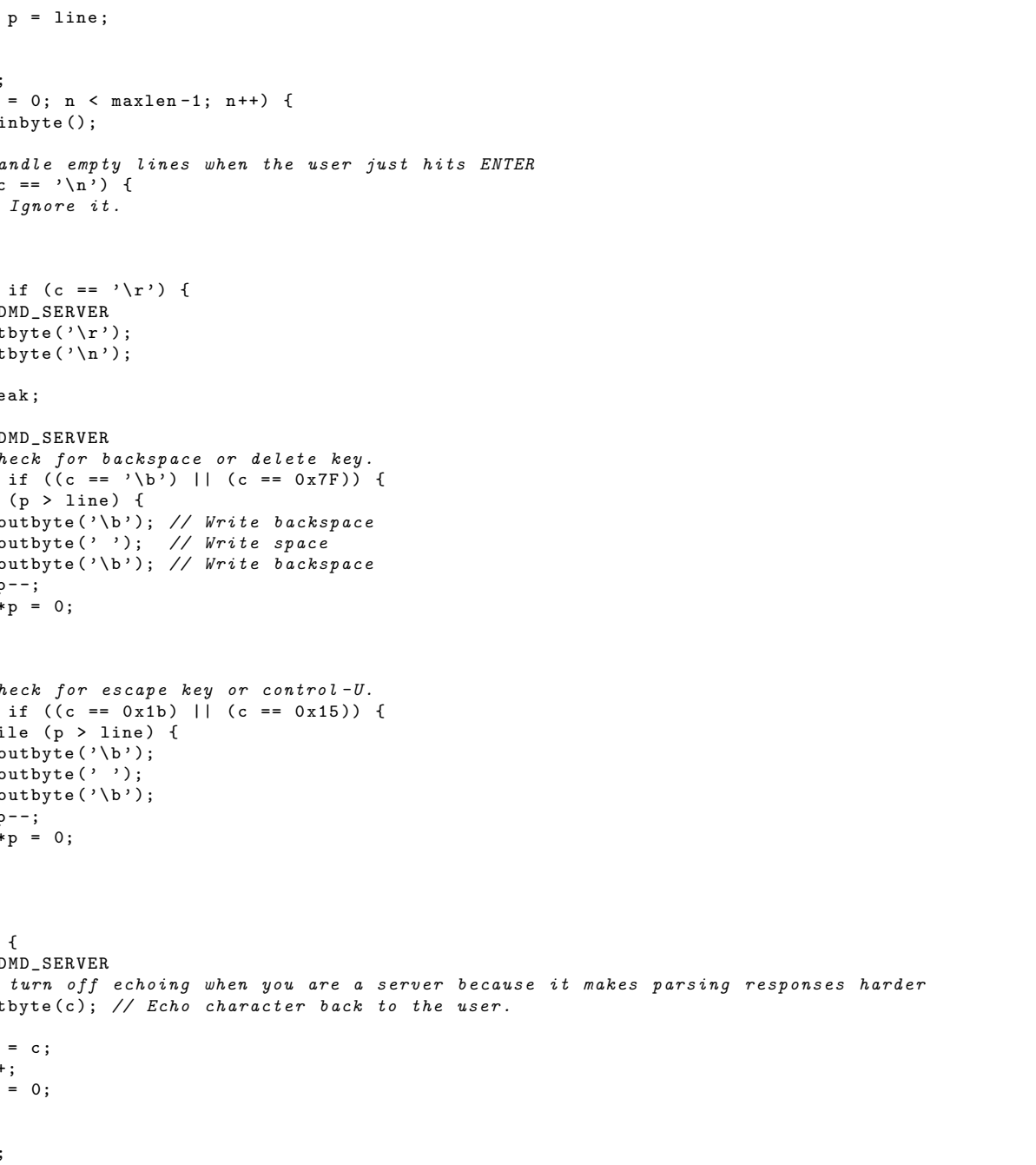


Listing B.12: Logic Allocation Lexer.

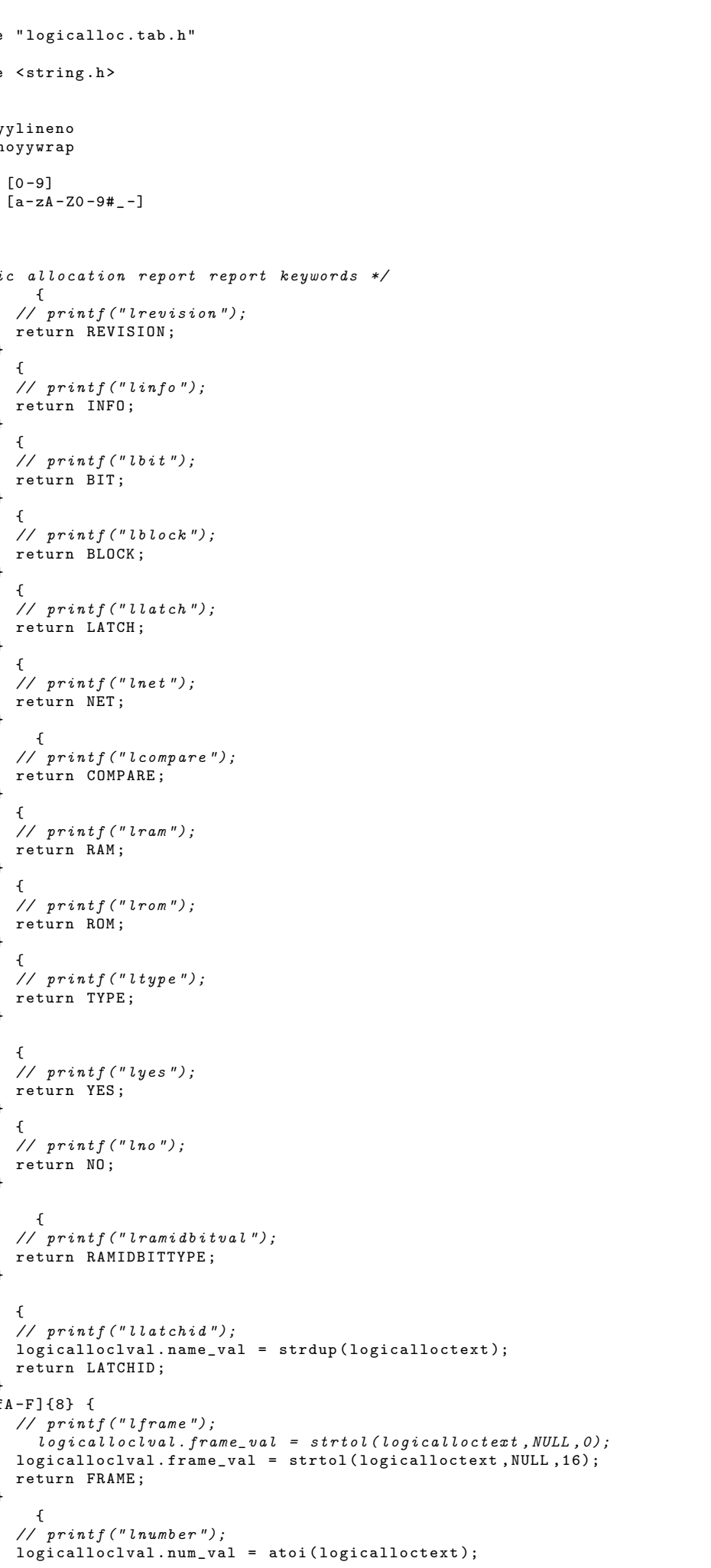


Yousef S. Iskander

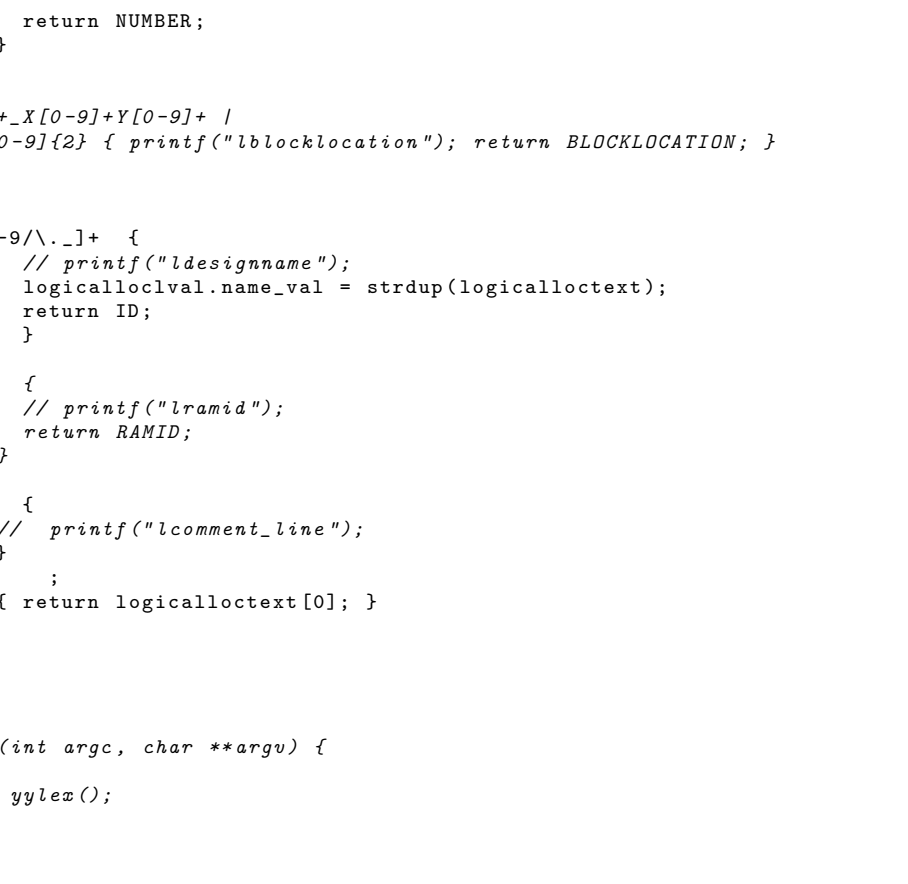


Listing B.13: Logic Allocation Parser.

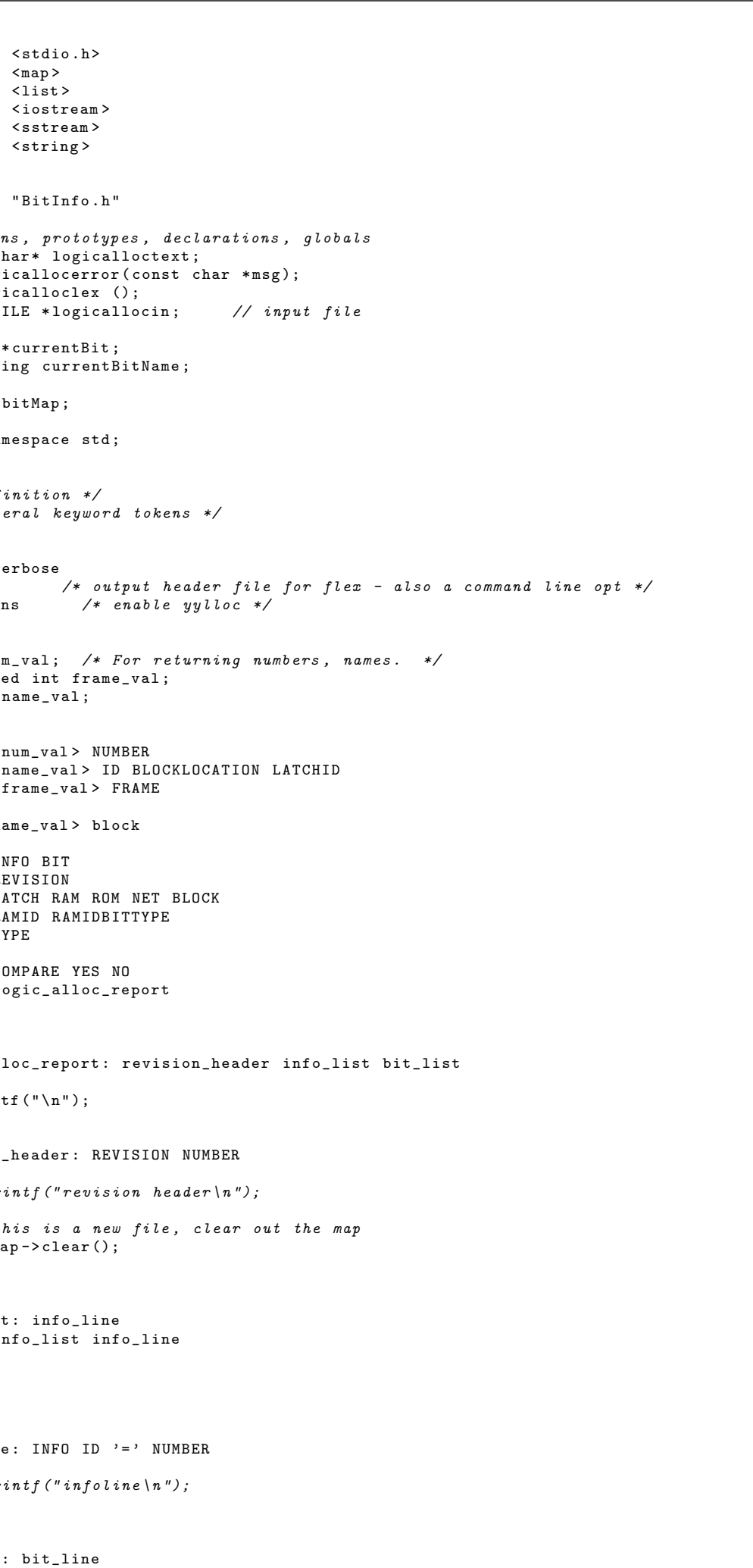




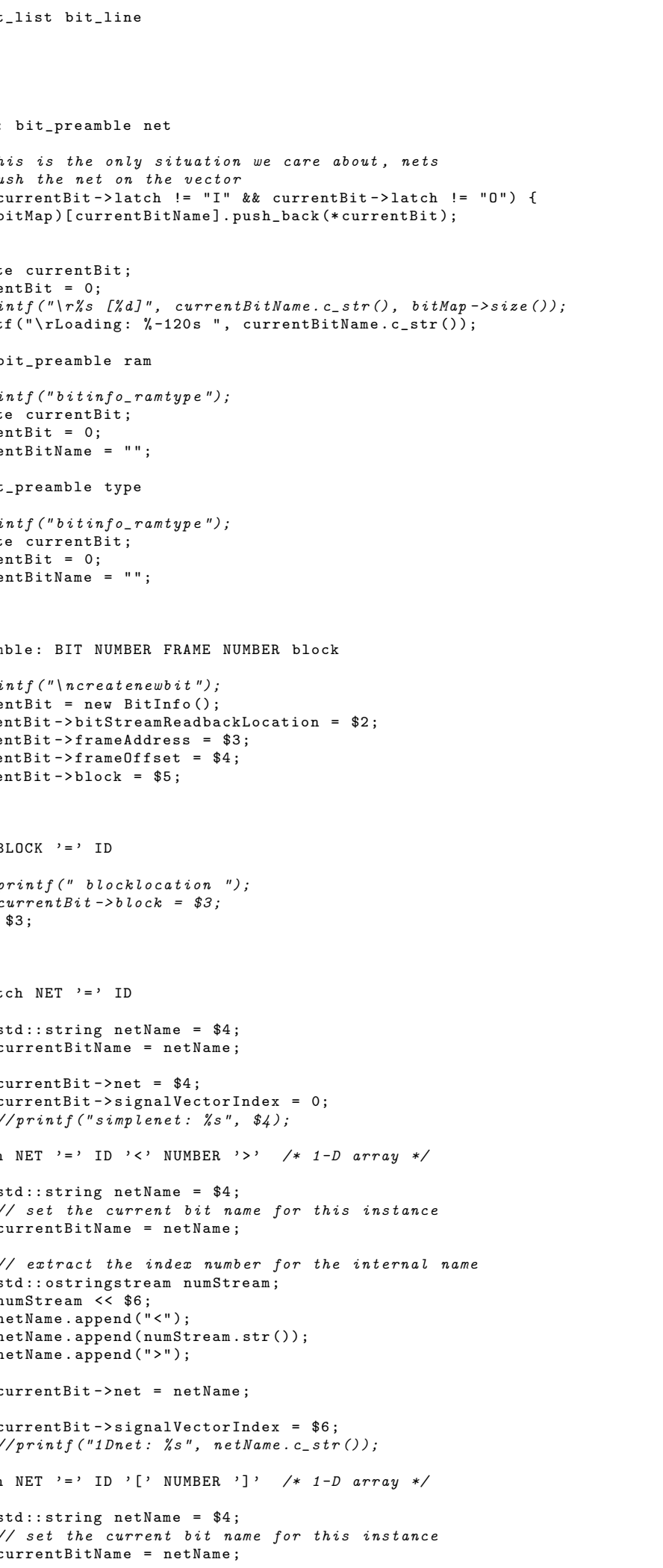




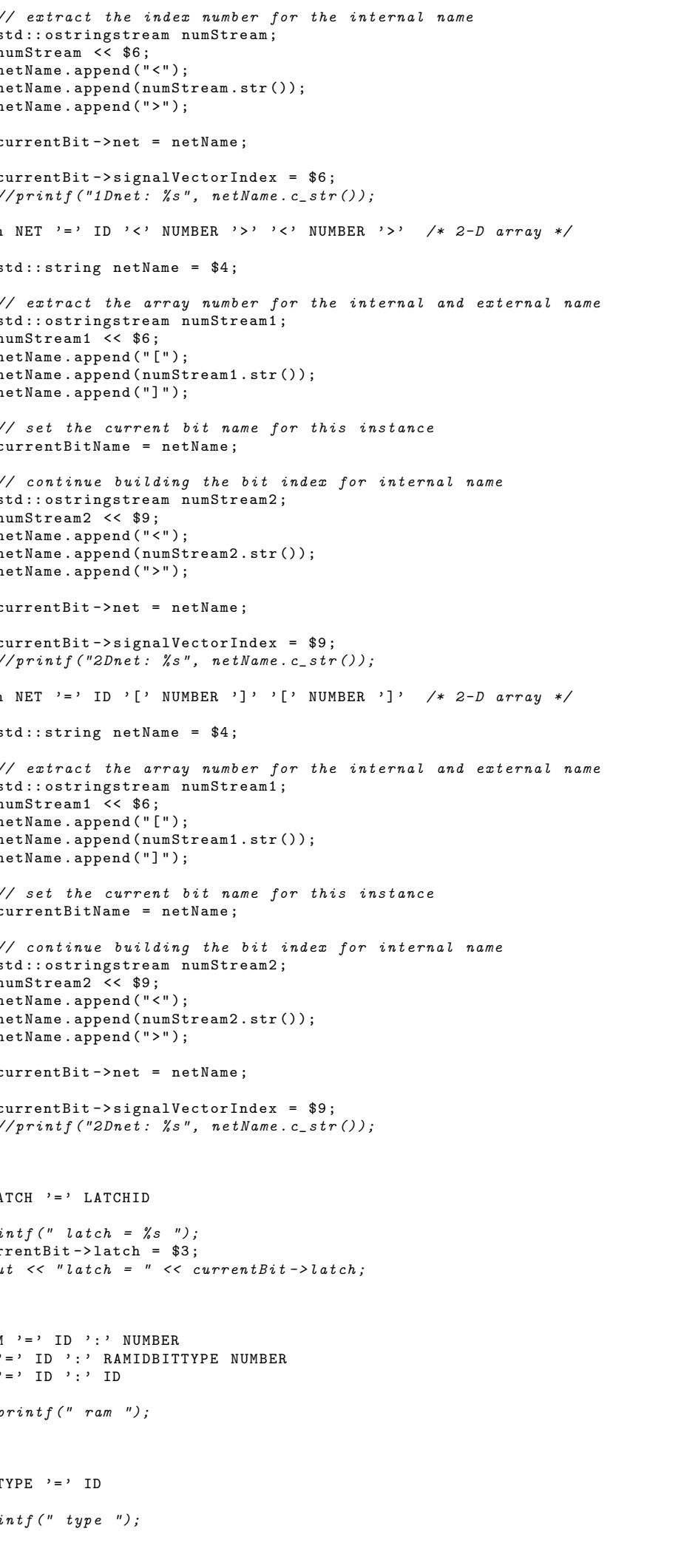




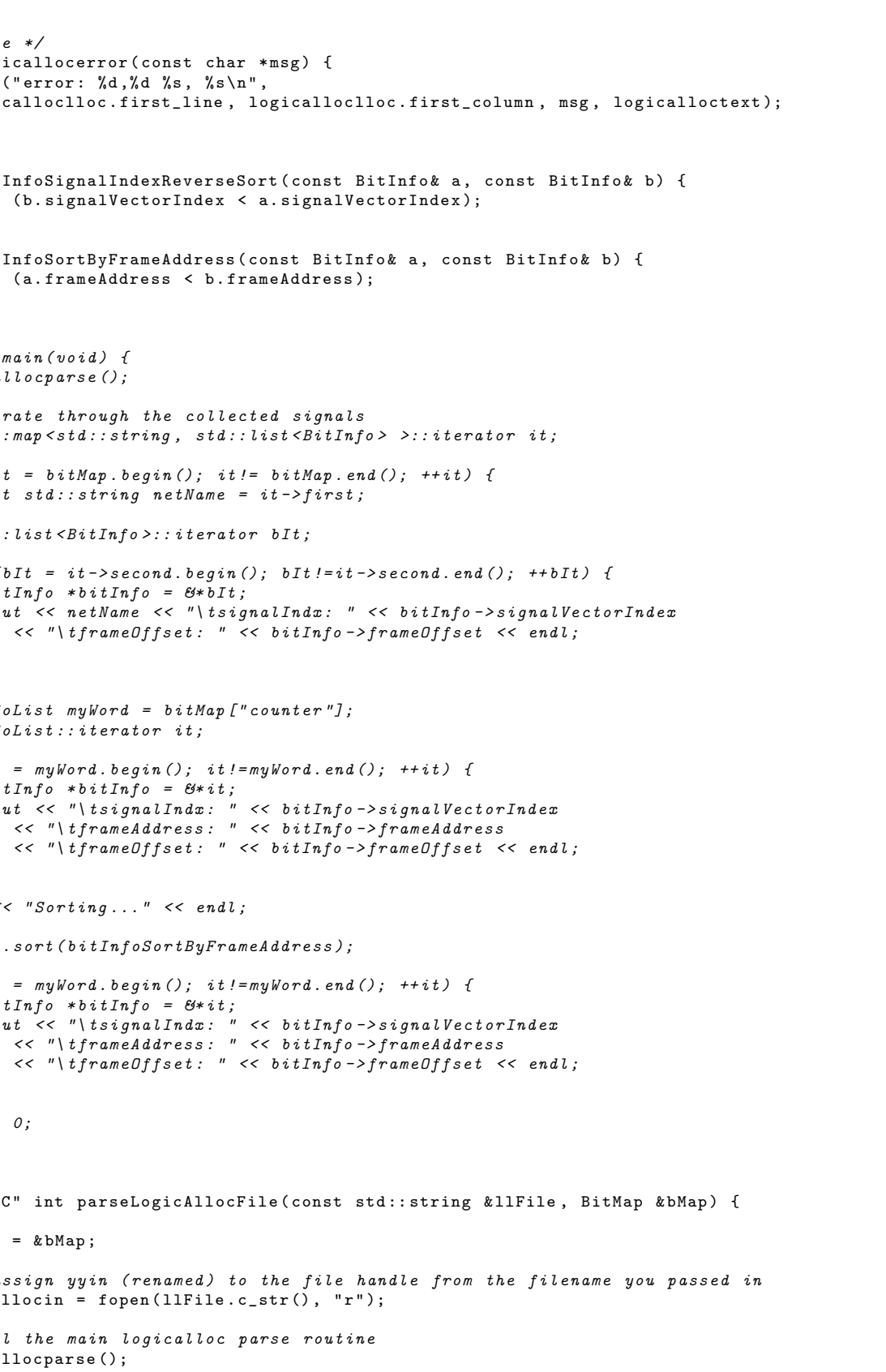


Listing B.14: Programmable Debug Controller.

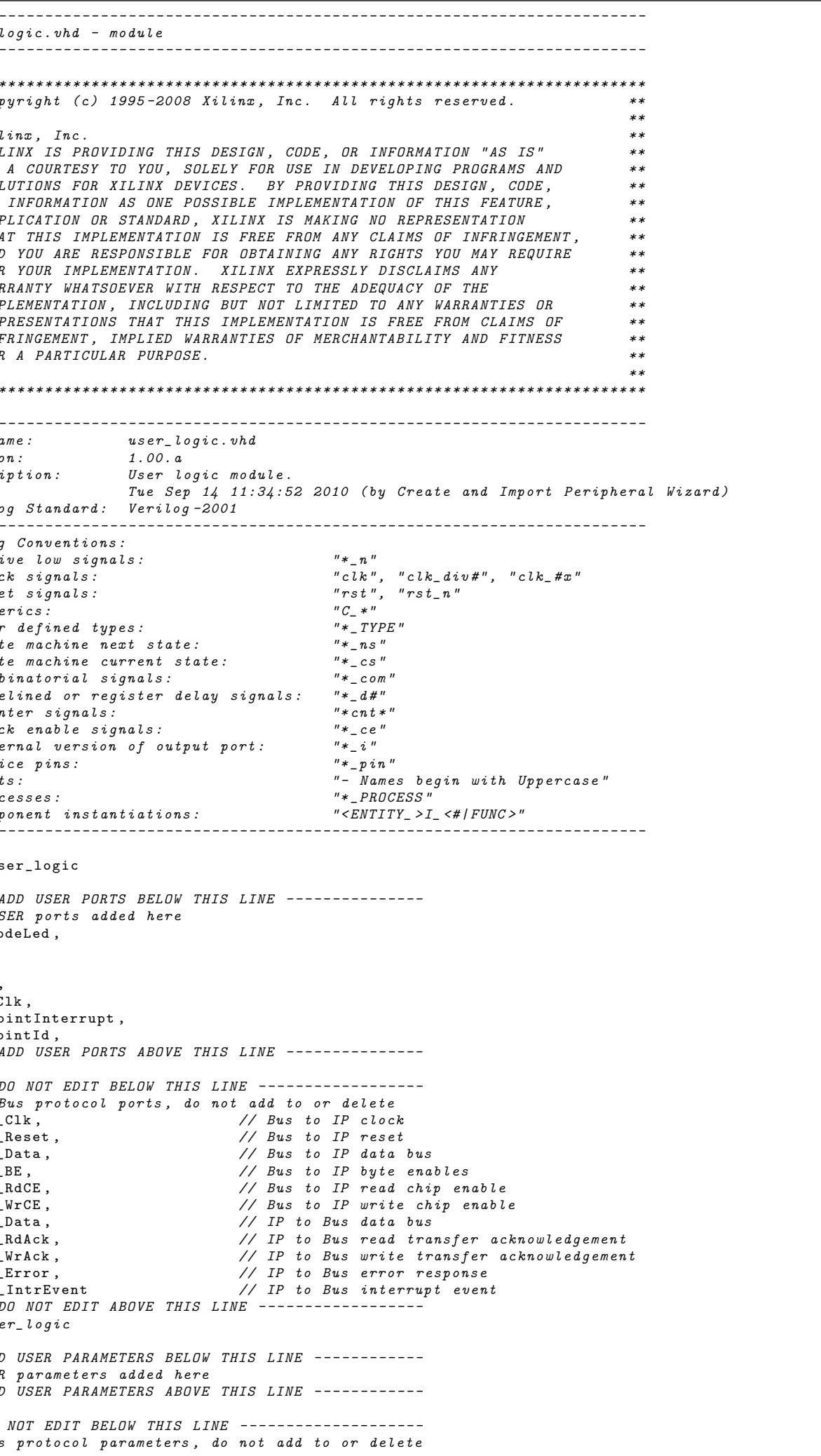




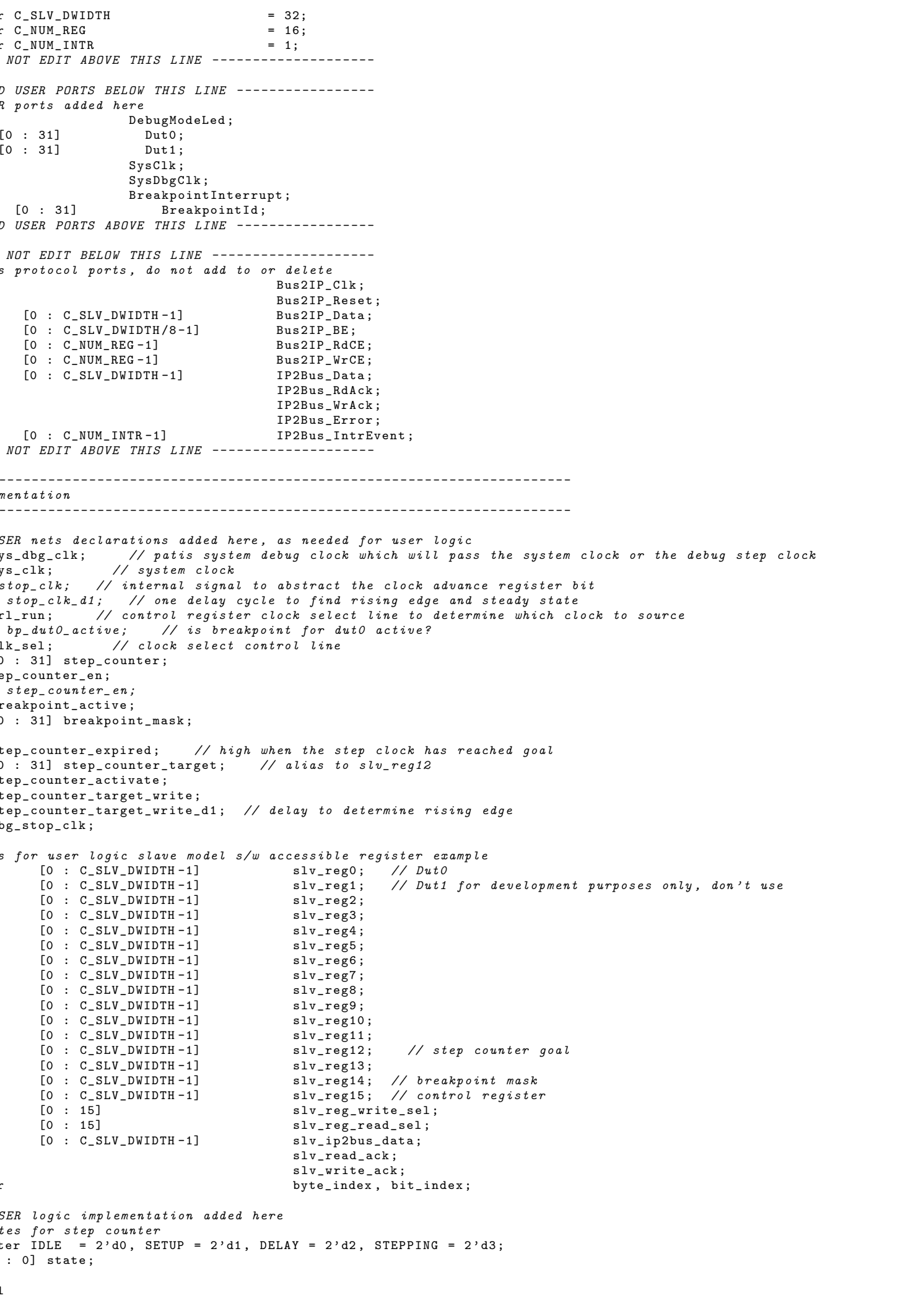




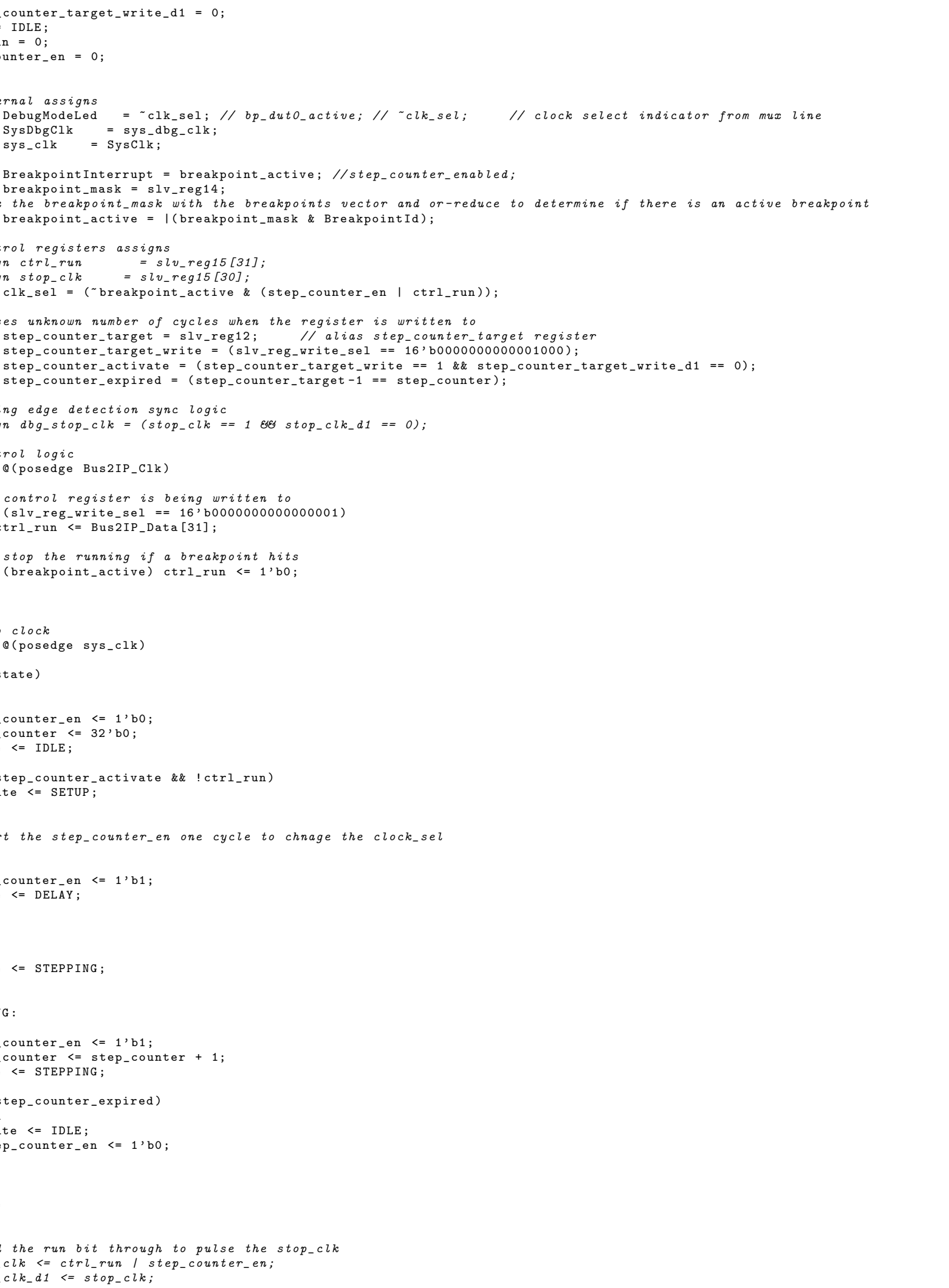




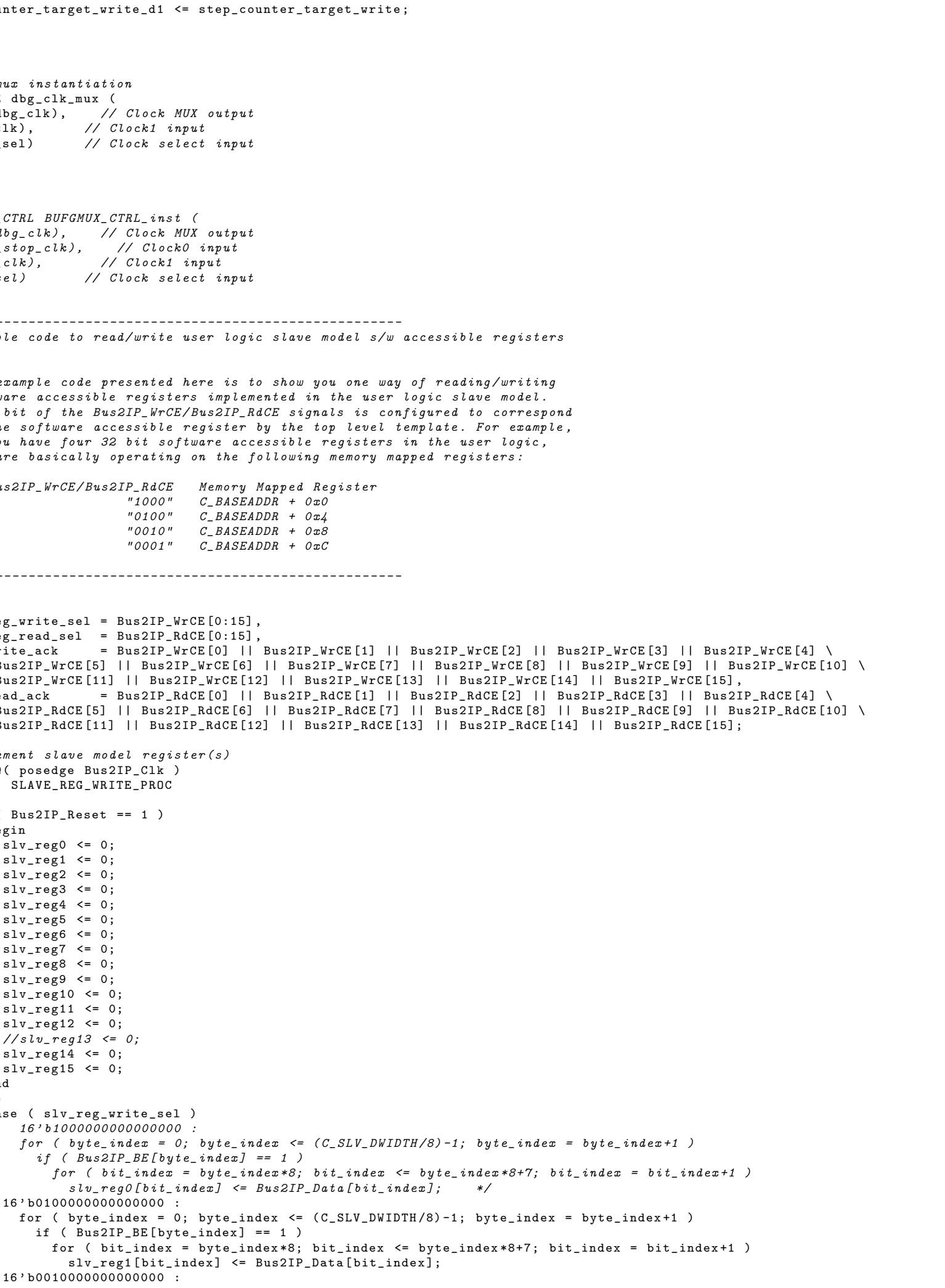




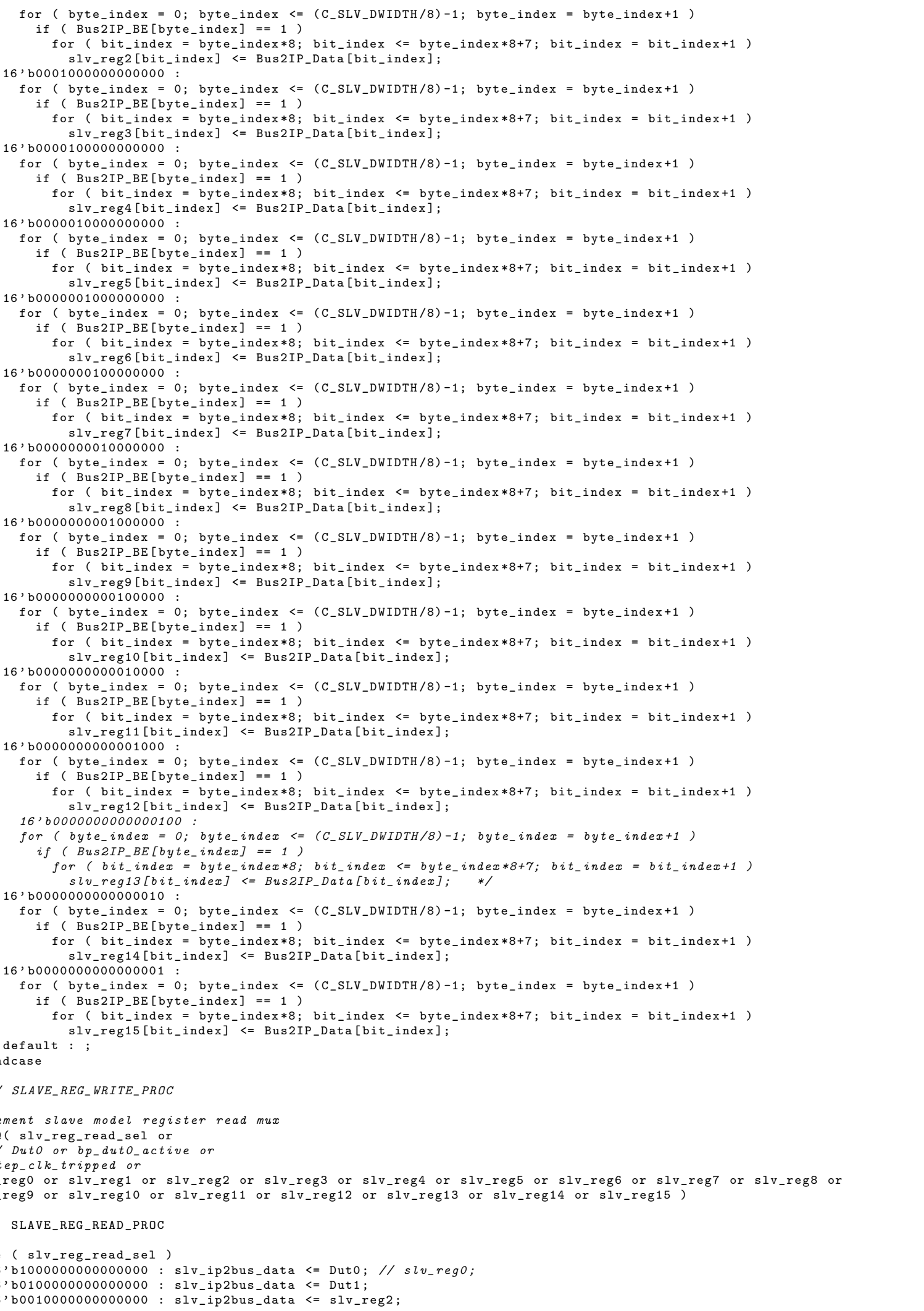


Yousef S. Iskander

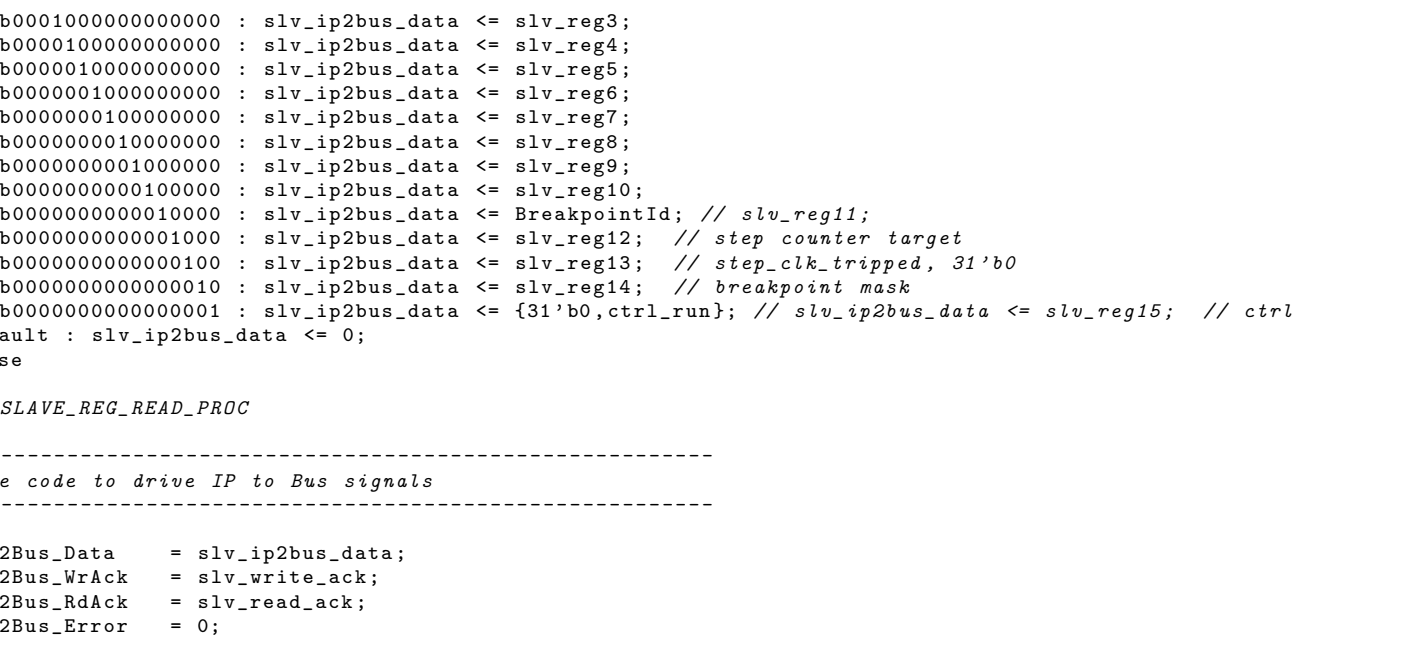


Listing B.15: Example of Generated Breakpoint Logic.

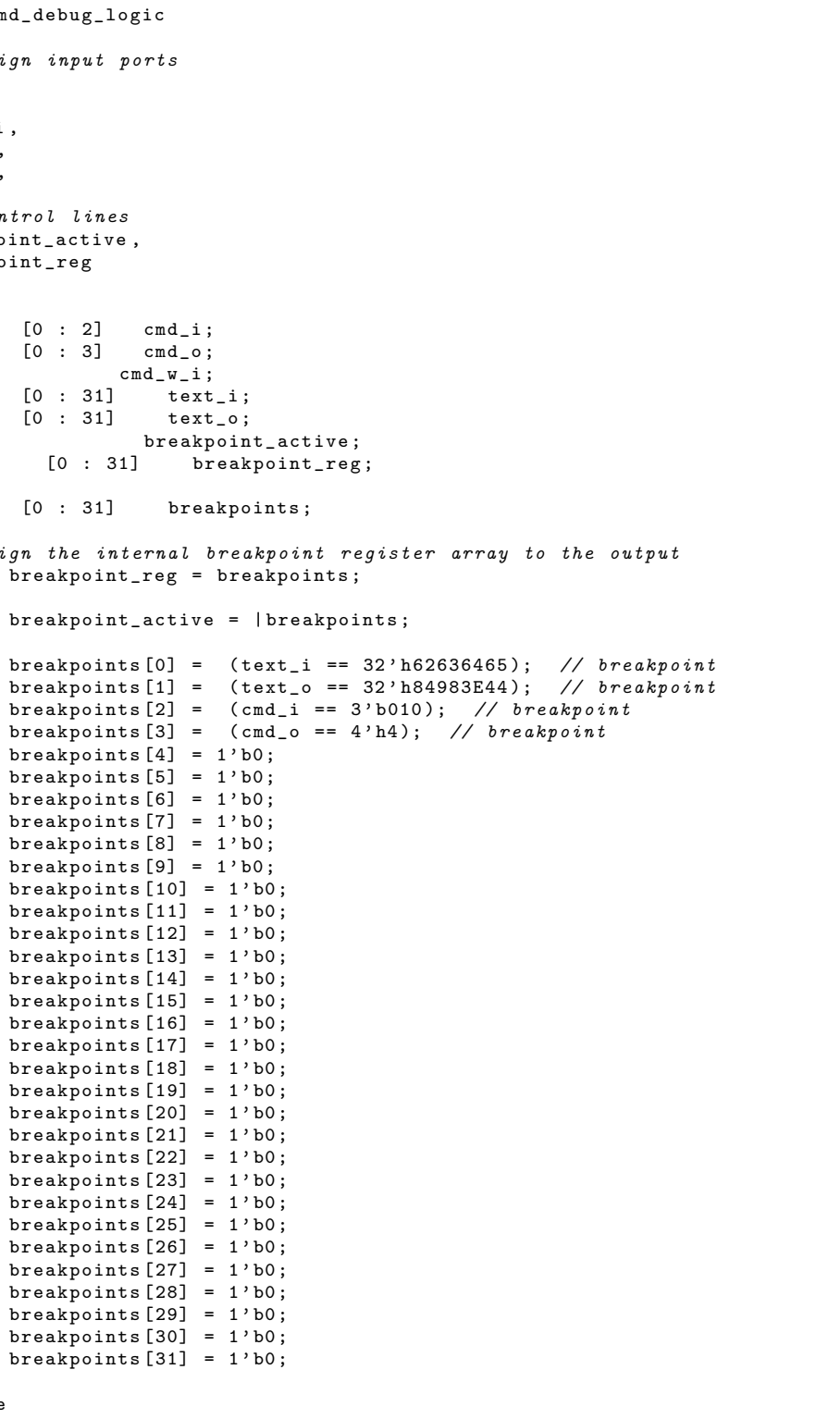

